\title{
Ultraprodutos métricos e normados e ultrassomas topológicas
}

\author{
João Paulo Cirineu de Jesus
}

\author{
TESE APRESENTADA AO \\ Instituto de Matemática e Estatística \\ da Universidade de São Paulo \\ PARA OBTENÇÃO DO TÍTULO DE \\ DOUTOR EM CIÊNCIAS
}

Programa: Matemática

Orientador: Prof. Dr. Rogério Augusto dos Santos Fajardo

São Paulo

15 de julho de 2021 



\title{
Ultraprodutos métricos e normados e ultrassomas topológicas
}

\author{
Esta versão da tese contém as correções e alterações sugeridas \\ pela Comissão Julgadora durante a defesa da versão original do trabalho, \\ realizada em 15/07/2021. Uma cópia da versão original está disponível no \\ Instituto de Matemática e Estatística da Universidade de São Paulo.
}

Comissão Julgadora:

- Prof. Dr. Rogério Augusto dos Santos Fajardo (orientador) - IME-USP

- Prof. Dr. Hugo Luiz Mariano - IME-USP

- Prof. Dr. Vladimir Pestov - UFSC

- Prof. Dr. Jorge Petrúcio Viana - UFF

- Prof. Dr. Marcelo Esteban Coniglio - UNICAMP 
Autorizo a reprodução e divulgação total ou parcial deste trabalho, por qualquer meio convencional ou eletrônico, para fins de estudo e pesquisa, desde que citada a fonte. 

À minha mãe, Marivalda, às minhas sobrinhas, Yasmin e Elizabeth e ao meu sobrinho, Yan. 



\section{Agradecimentos}

Primeiro, agradeço à minha família por todo apoio me deu para que eu chegsse ao nível acadêmico e profissional em que me encontro, de forma honesta e devida. Em especial, agradeço à minha mãe por seu amor e dedicação incondicionais e pelo suporte financeiro e incentivo dados a mim, que foram indispensáveis para que eu me pudesse me dedicar à obtenção do título que tanto almejei.

Segundo, agradeço ao Prof. Rogério Augusto dos Santos Fajardo pela compreensão e paciência durante todo o período em que me orientou e em todos os semestres em que me supervisionou como estagiário docente ou monitor. Também agradeço ao Prof. Hugo Luiz Mariano e ao Prof. Odilon Otávio Luciano pelo apoio que me deram em determinados momentos do meu doutoramento.

Terceiro, agradeço aos meus caros amigos de irmandade, Edgard Upinho e Márcio Paim, pelo apoio moral que deram a mim durante minha jornada rumo à conquista do meu título. Também agradeço aos caros colegas da B-141 do IME-USP, Hugo Ribeiro e Yuri Flores, pelo companheirismo até o final dessa jornada.

Agora, agradeço à Comissão Julgadora pelas sugestões dadas a mim durante minha defesa, as quais foram de grande valia para o melhoramento desta tese e serão muito úteis para o desenvolvimento de trabalhos futuros.

Por fim, agradeço à SAS-USP pelo apoio moradia que me concedeu durante todo o período do meu doutoramento e à UNIVESP pela concessão de bolsa do Programa de Cooperação USP/UNIVESP durante os dois últimos anos desse período. 



\section{Resumo}

JESUS, J. P. C. Ultraprodutos métricos e normados e ultrassomas topológicas. 2021. Tese (Doutorado em Matemática) - Instituto de Matemática e Estatística, Universidade de São Paulo, São Paulo, 2021. 153 p.

Neste trabalho, são estabelecidas generalizações de definições e resultados da teoria dos ultraprodutos de espaços métricos pontuados, no contexto dos espaços pseudométricos, e da teoria dos ultraprodutos de espaços normados e de Banach reais ou complexos, no contexto dos espaços pseudonormados sobre subcorpos quaisquer do corpo dos números complexos. Na teoria dos conjuntos de Zermelo-Fraenkel com o Teorema do Ultrafiltro, é apresentada uma introdução detalhada à teoria das ultrassomas de espaços topológicos de Tychonoff. Na teoria dos conjuntos de Zermelo-Fraenkel com o Axioma da Escolha, é demonstrada uma generalização do resultado de estabilidade sob ultraprodutos da classe dos espaços de funções reais contínuas que estão definidas em algum espaço topológico compacto e de Hausdorff.

Palavras-chave: Ultrafiltros. Pseudométricas. Pseudonormas. Ultraprodutos métricos. Ultraprodutos normados. Ultrassomas topológicas. 



\section{Abstract}

JESUS, J. P. C. Normed and metric ultraproducts and topological ultrasums. 2021. Tese (Doutorado em Matemática) - Instituto de Matemática e Estatística, Universidade de São Paulo, São Paulo, 2021. 153 p.

In this work, we establish a generalization of definitions and results from the theory of ultraproducts of pointed metric spaces in the setting of pseudometric spaces and from the theory of ultraproducts of real or complex normed spaces in the setting of pseudonormed spaces over any subfields of the field of complex numbers. We present in the Zermelo-Fraenkel set theory with the Ultrafilter Theorem a detailed introdution to the theory of ultrasums of Tychonoff topological spaces. We proof in the ZermeloFraenkel set theory with the Axiom of Choice a generalization of the result of stability under ultraproducts of the class of spaces of continuous real-valued functions which are defined on some Hausdorff and compact topological space.

Keywords: Ultrafilters. Pseudometrics. Pseudonorms. Metric ultraproducts. Normed ultraproducts. Topological ultrasums. 



\section{Lista de Diagramas}

1.1 Diagrama $i .1 \ldots \ldots \ldots \ldots \ldots \ldots$

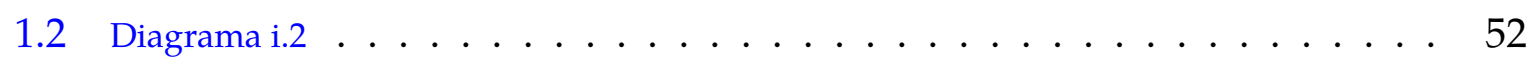

3.1 Diagrama iii.1 . . . . . . . . . . . . . . . . . . . 123

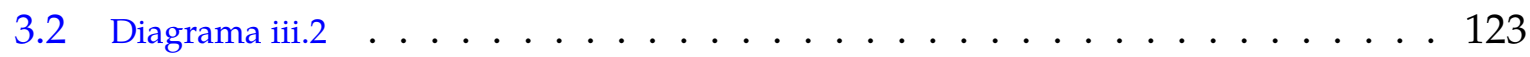

3.3 Diagrama iii.3 . . . . . . . . . . . . . . . . . . 124

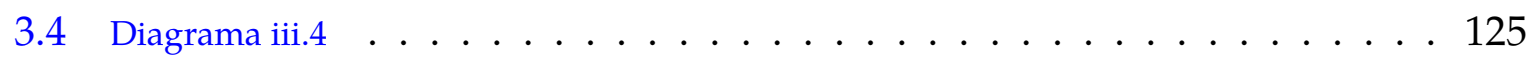

3.5 Diagrama iii.5 . . . . . . . . . . . . . . . . . . 132

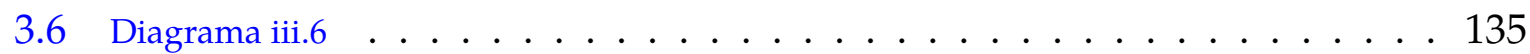

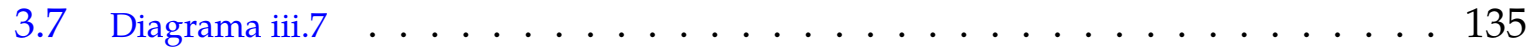

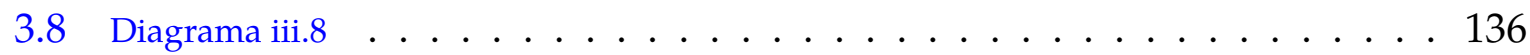





\section{Lista de Símbolos}

Símbolos de relações e operadores

$\subseteq \quad$ Relação de inclusão

$\cup \quad$ Operador conjunto união

$\cap$ Operador conjunto interseção (de conjuntos não vazios)

Operador conjunto diferença

$\mathcal{P} \quad$ Operador conjunto potência

im Operador conjunto imagem

id Operador função identidade

Símbolos de conjuntos e estruturas

$\emptyset \quad$ Conjunto vazio

$\omega \quad$ Conjunto dos números naturais (de von Neumann)

$\mathbb{R} \quad$ Conjunto ou corpo dos números reais

$\mathbb{R}^{+} \quad$ Conjunto dos números reais positivos

$\mathbb{C}$ Conjunto ou corpo dos números complexos

Símbolos de indicadores

- Indicador de final de definição 
- Indicador de final de prova e de enunciado sem prova

$\square \quad$ Indicador de final de comentário e de apresentação de exemplo

$\bigcirc \quad$ Indicador de prova deixada como exercício para o leitor 


\section{Sumário}

$\begin{array}{ll}\text { Introdução } & 1\end{array}$

1 Métricas generalizadas e pseudonormas 5

1.1 Noções conjuntistas e topológicas . . . . . . . . . . . . . . . . . . . . . . . 5

1.2 Noções métricas e analítico-funcionais . . . . . . . . . . . . . . . . . . . . . . . 24

2 Ultraprodutos métricos e normados $\quad 59$

2.1 Convergência com respeito a filtros . . . . . . . . . . . . . . . . . . . . . . 59

2.2 Ultraprodutos de espaços pseudométricos e espaços pseudonormados . . . . . 81

3 Ultrassomas topológicas $\quad 121$

3.1 Ultrassomas de espaços topológicos de Tychonoff . . . . . . . . . . . . . . . 121

3.2 Ultraprodutos normados de espaços de funções reais contínuas e limitadas que estão definidas em espaços topológicos de Tychonoff. . . . . . . . . . . . . . . 138

$\begin{array}{lr}\text { Considerações Finais } & 141\end{array}$

$\begin{array}{ll}\text { Referências Bibliográficas } & 143\end{array}$

Índice Remissivo $\quad 149$ 



\section{Introdução}

A definição de ultraproduto foi originalmente introduzida, no contexto das álgebras universais, por J. Łoś em [Los1955]. No entanto, construções de ultraprodutos já vinham aparecendo, de alguma forma, em trabalhos publicados anteriormente - por exemplo, de K. Gödel ([G1930]), de T. Skolem ([Sko1934]), de E. Hewitt ([Hew1948]) (cf. [BeS1969, seç. 5.6 e 12.8]) e de F. B. Wright ([W1954]) (cf. [She2009, seç. 1]).

A definição de ultraproduto de espaços normados e de Banach é uma adaptação da definição de ultraproduto de estruturas de primeira ordem e foi originalmente introduzida por D. Dacunha-Castelle e J.-L. Krivine em [DCK1972]. ${ }^{1}$ Mas, sua restrição aos espaços $L_{p}(p \in[1,+\infty[)$ aparece anteriormente na tese de doutorado de Krivine ([Kri1967]). O principal motivo para introduzi-la foi estabelecer uma caracterização dos reticulados de Banach que são isomorfos a um subespaço vetorial de algum espaço $L_{p}(p \in[1,+\infty[)$ por meio dos subespaços vetoriais de dimensão finita desses reticulados.

As construções de ultraprodutos de espaços normados e de Banach passaram a ter uma grande importância na Análise Funcional, visto que tais construções se tornaram um ferramental amplamente utilizado tanto nas teorias da representabilidade finita, da dualidade, dos ideais de operadores e da aproximação local quanto na teoria isométrica dos espaços de Banach, como pode ser visto em trabalhos já consagrados - por exemplo,

${ }^{1}$ É interessante destacar que, no contexto das estruturas não standard de ordem superior, foi apresentada por W. A. J. Luxemburg, em [Lux1969], uma construção que tem como caso particular a de ultrapotência de espaços normados e de Banach. Tal construção é a de envelope não standard de espaços uniformes - e referente isto, tem-se a asserção

o envelope não standard e a ultrapotência de um espaço normado com respeito a um mesmo ultrafiltro são isometricamente isomorfos, que se encontra, por exemplo, em [HI2002, p. 20]. 
de J. Stern ([S1978]) e de S. Heinrich ([Hei1980]) - ou publicados em anos recentes - por exemplo, de A. Avilés et al. ([Avi+2 2013] e [Avi ${ }^{+}$2016, cap. 4]).

O uso cada vez mais frequente de técnicas inspiradas em métodos modelo-teóricos para a resolução de problemas da Análise Funcional, motivou C. W. Henson e J. Iovino a desenvolverem, em [HI2002], uma teoria dos modelos da lógica de fórmulas positivamente limitadas com semântica aproximada para estruturas de espaços normados. Um pouco depois, I. Ben Yaacov et al. desenvolveram, em [ $\left.\mathrm{B}^{+} 2008\right]$, uma teoria dos modelos de uma lógica contínua de primeira ordem para estruturas métricas, na qual foram estabelecidos diversos resultados análogos a outros apresentados em [HI2002].

Com o estudo sobre a estabilidade sob ultraprodutos de classes de espaços de Banach, surgiu a noção de ultracoproduto topológico, ${ }^{2}$ o que, em termos analítico-funcionais, pode ser descrito como

um espaço topológico compacto e de Hausdorff tal que o espaço de funções reais contínuas que estão definidas nesse espaço é isometricamente isomorfo ao ultraproduto de uma família de espaços de funções reais contínuas que estão definidas em algum espaço topológico compacto e de Hausdorff.

A existência de ultracoprodutos topológicos pode ser justificada utilizando o Teorema da Representação de Gelfand para álgebras $C^{*}$ comutativas e unitárias - como é feito em [Hei1980, seç. 3]. Com uma abordagem muito mais topológica, P. Bankston apresentou outra definição de ultracoproduto topológico em [Ban1984, seç. 3] e [Ban1987, seç. 1]. Posteriormente, R. Gurevič apresentou um resultado (Proposição 2 de [Gur1988]) com o qual se estabelece que a definição de Bankston e a descrição analítico-funcional acima determinam espaços topológicos homeomorfos.

No que segue, são descritos brevemente os capítulos desta tese, deixando claro ao que se propõe cada um e destacando resultados que são contribuições nossas com este trabalho:

No Capítulo 1, são apresentadas as noções e proposições conjuntistas, topológicas e analítico-funcionais que são necessárias para a compreensão e o desenvolvimento desta tese. Os seguintes resultados são contribuições dadas nesse capítulo: os Teoremas 1.2.13 e 1.2.31.

No Capítulo 2, são estabelecidas, na teoria dos conjuntos de Zermelo-Fraenkel

${ }^{2}$ Esta é a expressão utilizada na literatura a que tivemos acesso. Nesta tese, iremos adotar a expressão ultrassoma topológica em seu lugar. 
ou em particulares extensões próprias desta teoria, generalizações de definições e resultados que podem ser encontrados nas referências adotadas sobre ultraprodutos de espaços métricos ou sobre ultraprodutos de espaços normados e de Banach. Essas generalizações são estabelecidas com a introdução da definição de conjunto finitamente distal com respeito a uma família de pré-métricas. Os seguintes resultados são contribuições dadas nesse capítulo: os Teoremas 2.1.3, 2.1.16, 2.1.25, 2.2.6, 2.2.9, 2.2.10, 2.2.11, 2.2.15, 2.2.17 e 2.2.30 e os Exemplos 2.1.9 e 2.1.17.

No Capítulo 3, é introduzida detalhadamente, na teoria dos conjuntos de ZermeloFraenkel com o Teorema do Ultrafiltro, a teoria das ultrassomas de espaços topológicos de Tychonoff. Também é demonstrada, na teoria dos conjuntos de Zermelo-Fraenkel com o Axioma da Escolha, uma generalização da Proposição 2 de [Gur1988] no contexto dos espaços topológicos de Tychonoff. Os seguintes resultados são contribuições dadas nesse capítulo: os Teoremas 3.1.5 e 3.2.3.

Por fim, informamos que, ao longo desta tese, as siglas ZF e AC irão representar, respectivamente, a teoria dos conjuntos de Zermelo-Fraenkel e o Axioma da Escolha, enquanto as siglas $\mathbf{Z F C}$ e $\mathbf{A C}_{\omega}$ irão representar, respectivamente, a teoria $\mathbf{Z F}+\mathbf{A C}$ e a restrição de $\mathbf{A C}$ às famílias enumeráveis de conjuntos não vazios. Exceto por menção explícita em contrário, quaisquer definições, asserções, comentários e exemplos neste trabalho podem ser estabelecidos em ZF - cuja consistência é equivalente à de ZFC e está sendo assumida em toda esta tese. 



\section{CAPÍTULO 1}

\section{Métricas generalizadas e pseudonormas}

Neste capítulo, são apresentadas as noções e proposições conjuntistas, topológicas e analítico-funcionais que são necessárias para a compreensão e o desenvolvimento do que é abordado nos próximos capítulos. Para a terminologia e notações que serão adotadas ao longo deste capítulo e dos demais, seguiremos os livros-textos [End1977, Jec2003] de Teoria dos Conjuntos, [Wil1970, Eng1989] de Topologia Geral e [Meg1998, Con1990] de Análise Funcional.

\subsection{Noções conjuntistas e topológicas}

Nesta seção, são apresentados enunciados precisos - com demonstração ou não de resultados conjuntistas e topológicos que serão utilizados nos próximos capítulos.

Definição 1.1.1. Sejam $I$ um conjunto e $\mathcal{F} \subseteq \mathcal{P}(I)$. Diremos que:

(i) $\mathcal{F}$ é uma cobertura de $I$ se $\cup \mathcal{F}=I$.

(ii) $\mathcal{F}$ tem a propriedade da interseção finita se valer a seguinte condição: para todo $\mathcal{A} \subseteq \mathcal{F}$, se $\mathcal{A}$ for não vazio e finito, então o conjunto $\cap \mathcal{A}$ é não vazio.

Usaremos a sigla PIF para a expressão propriedade da interseção finita. Suponhamos que I seja não vazio. Diremos que:

(iii) $\mathcal{F}$ é um filtro (sobre $I$ ) se valerem as seguintes condições:

(1) $\emptyset \notin \mathcal{F}$. 
(2) $I \in \mathcal{F}$.

(3) Para quaisquer $A, B \in \mathcal{F}, A \cap B \in \mathcal{F}$.

(4) Para todo $A \in \mathcal{F}$ e todo $B \subseteq I$, se $A \subseteq B$, então $B \in \mathcal{F}$.

(iv) $\mathcal{F}$ é uma base de filtro (sobre $I$ ) se valerem as seguintes condições:

(1) $\emptyset \notin \mathcal{F}$.

(2) $\mathcal{F}$ é não vazio.

(3) Para quaisquer $A, B \in \mathcal{F}$, existe um $C \in \mathcal{F}$ tal que $C \subseteq A \cap B$.

(v) $\mathcal{F}$ é um ultrafiltro (sobre $I$ ) se valerem as seguintes condições:

(1) $\mathcal{F}$ é um filtro sobre $I$.

(2) Para todo $A \subseteq I, A \in \mathcal{F}$ ou $I \backslash A \in \mathcal{F}$.

(vi) $\mathcal{F}$ é um filtro maximal (sobre $I$ ) se valerem as seguintes condições:

(1) $\mathcal{F}$ é um filtro sobre $I$.

(2) Para todo filtro $\mathcal{G}$ sobre $I$, se $\mathcal{F} \subseteq \mathcal{G}$, então $\mathcal{F}=\mathcal{G}$.

Comentário 1.1.2. Dado um conjunto não vazio $I$ e um $\mathcal{F} \subseteq \mathcal{P}(I)$, é claro que:

(i) (1) (1) $\{I\}$ é um filtro sobre $I$.

(2) Se $\mathcal{F}$ for um filtro sobre $I$, então $\{I\} \subseteq \mathcal{F}$.

(2) Para todo $i \in I$, a família $\{\{i\}\}$ é uma base de filtro sobre $I$.

É fácil ver que:

(ii) (1) Se $\mathcal{F}$ for um filtro sobre $I$, então $\mathcal{F}$ é uma base de filtro sobre $I$.

(2) Se $\mathcal{F}$ for uma base de filtro sobre $I$, então $\mathcal{F}$ tem a PIF.

Também é fácil ver que:

(iii) Para todo $i \in I$, a família $\{A \in \mathcal{P}(I): i \in A\}$ é um ultrafiltro sobre $I$.

Para cada $i \in I$, a família em (iii) será chamada de o ultrafiltro principal sobre $I$ em $i$ e denotada por $\mathcal{U}_{I}(i)$ (ou, simplesmente, por $\mathcal{U}(i)$ ). Note que:

(iv) Para todo $i \in I, \mathcal{U}_{I}(i)$ é um filtro maximal. 
Suponhamos que $\mathcal{F}$ seja um filtro sobre $I$. Usando o item (iv), mostra-se facilmente que:

(v) Para todo $i \in I, \mathcal{F}=\mathcal{U}_{I}(i)$ se, e somente se, $\{i\} \in \mathcal{F}$.

É fácil concluir que:

(vi) Para todo $i \in I$, se $\mathcal{F}$ for um ultrafiltro, então $\mathcal{F}=\mathcal{U}_{I}(i)$ se, e somente se, $i \in \cap \mathcal{F}$. Também é fácil concluir que:

(vii) Se $I$ for infinito, então a família $\{A \in \mathcal{P}(I): I \backslash A$ é finito $\}$ é um filtro sobre $I$.

A família em (vii) será chamada de o filtro de Fréchet sobre I e denotada por Cofin(I). Dada uma topologia $\tau$ sobre $I$, é fácil verificar que:

(viii) A família $\{A \in \mathcal{P}(I): A$ é uma vizinhança de $i$ em $\langle I, \tau\rangle\}$ é um filtro sobre $I$.

A família em (viii) será chamada de o filtro das vizinhanças de $i$ em $\langle I, \tau\rangle$ e denotada por $\mathcal{N}_{\tau}(i)$ (ou, simplesmente, por $\left.\mathcal{N}(i)\right)$. Note que:

(ix) Para todo $i \in I$,

(1) $\mathcal{N}_{\tau}(i) \subseteq \mathcal{U}_{I}(i)$

(2) $\mathcal{N}_{\tau}(i) \cap \tau=\mathcal{U}_{I}(i) \cap \tau$.

(3) $\mathcal{N}_{\tau}(i)=\mathcal{U}_{I}(i)$ se, e somente se, $i$ for um ponto isolado de $\langle I, \tau\rangle$.

Comentário 1.1.3. Dados um conjunto $I$ e um $\mathcal{G} \subseteq \mathcal{P}(I)$, considere as seguintes famílias:

$$
\begin{aligned}
\mathcal{G}^{\bigcap} & :=\{\cap \mathcal{A}: \mathcal{A} \in \mathcal{P}(\mathcal{G}) \backslash\{\emptyset\} \wedge \mathcal{A} \text { é finito }\} \\
& \mathrm{e} \\
\mathcal{G}^{\subseteq} & :=\{A \in \mathcal{P}(I): \exists B \in \mathcal{G}(B \subseteq A)\} .
\end{aligned}
$$

Note que:

(i) $\mathcal{G} \subseteq \mathcal{G}^{\cap} \cap \mathcal{G}^{\subseteq}$.

Suponha que I seja não vazio. Verifica-se corriqueiramente que:

(ii) (1) Se $\mathcal{G}$ for não vazio e tiver a PIF, então $\mathcal{G}^{\bigcap}$ é uma base de filtro sobre $I$.

(2) Se $\mathcal{G}$ for um filtro sobre $I$, então $\mathcal{G}^{\bigcap}=\mathcal{G}$.

(iii) Se existir um filtro $\mathcal{F}$ sobre $I$ tal que $\mathcal{G} \subseteq \mathcal{F}$, então 
(1) $\mathcal{G}^{\subseteq} \subseteq \bigcap\{\mathcal{A} \in \mathcal{P}(\mathcal{P}(I)): \mathcal{G} \subseteq \mathcal{A} \wedge \mathcal{A}$ é um filtro sobre $I\}$.

(2) $\mathcal{G}^{\subseteq}$ é um filtro sobre $I$ se, e somente se,

$$
\mathcal{G}^{\subseteq}=\bigcap\{\mathcal{A} \in \mathcal{P}(\mathcal{P}(I)): \mathcal{G} \subseteq \mathcal{A} \wedge \mathcal{A} \text { é um filtro sobre } I\} .
$$

(iv) (1) Se $\mathcal{G}$ for uma base de filtro sobre $I$, então $\mathcal{G}^{\complement}$ é um filtro sobre $I$.

(2) Se $\mathcal{G}$ for um filtro sobre $I$, então $\mathcal{G}^{\subseteq}=\mathcal{G}$.

Caso $\mathcal{G}$ seja uma base de filtro sobre $I$, a família $\mathcal{G} \subseteq$ será chamada de o filtro gerado por $\mathcal{G}$ (sobre I). Com o uso dos itens (i) e (iv) (1) acima e dos itens (i) (2) e (v) do Comentário 1.1.2, conclui-se que:

(v) Para todo $i \in I,\{\{i\}\}^{\subseteq}=\mathcal{U}_{I}(i)$.

Suponha que $\mathcal{G}$ seja não vazio e tenha a PIF. Mostra-se facilmente que:

(vi) (1) Para todo $A \in \mathcal{P}(I) \backslash\left(\mathcal{G}^{\bigcap}\right)^{\subseteq}$, a familia $\mathcal{G} \cup\{I \backslash A\}$ tem a PIF.

(2) Se $\mathcal{G}$ for uma base de filtro sobre $I$, então, para todo $A \in \mathcal{P}(I) \backslash \mathcal{G}^{\subseteq}$, a familia $\mathcal{G} \cup\{I \backslash A\}$ tem a PIF.

(3) Se $\mathcal{G}$ for um filtro sobre $I$, então, para todo $A \in \mathcal{P}(I) \backslash \mathcal{G}$, a familia $\mathcal{G} \cup\{I \backslash A\}$ tem a PIF.

Suponhamos que $\mathcal{G}$ seja um filtro sobre $I$. Mostra-se que:

(vii) $\mathcal{G}$ é um ultrafiltro se, e somente se, $\mathcal{G}$ for um filtro maximal.

Suponha que I seja finito. Utilizando a finitude da família $\mathcal{P}(I)$ e usando o itens (iii) e (vi) do Comentário 1.1.2, conclui-se que:

(viii) (1) O conjunto $\bigcap \mathcal{G}$ é não vazio.

(2) $\mathcal{G}$ é um ultrafiltro se, e somente se, existir um $i \in I$ tal que $\mathcal{G}=\mathcal{U}_{I}(i)$.

Definição 1.1.4. Sejam $I$ um conjunto infinito e $\mathcal{F}$ um filtro sobre $I$. Diremos que:

(i) $\mathcal{F}$ é livre (sobre $I)$ se $\bigcap \mathcal{F}=\emptyset$.

(ii) $\mathcal{F}$ é um ultrafiltro principal (sobre $I$ ) se valer a seguinte condição: existe um $i \in I$ tal que $\mathcal{F}=\mathcal{U}_{I}(i)$ 
Proposição 1.1.5. Sejam I um conjunto infinito e $\mathcal{U}$ um ultrafiltro sobre I. Então, são equivalentes:

(i) $\mathcal{U}$ é livre.

(ii) $\mathcal{U}$ não é um ultrafiltro principal.

(iii) $\operatorname{Cofin}(I) \subseteq \mathcal{U}$.

(iv) Para todo $A \in \mathcal{U}$, A é infinito.

(v) Para todo $A \in \mathcal{U}$, A não é unitário.

Um princípio maximal de grande importância para a Lógica Matemática, a Teoria dos Conjuntos e a Topologia Geral é o Teorema do Ultrafiltro, notadamente pelo fato deste último ser equivalente, em ZF, aos seguintes princípios: o Teorema da Compacidade para linguagens de primeira ordem (v. [Sch1997, cap. 14, seç. 61]), o Teorema da Representação de Stone para álgebras de Boole (v. [Sch1997, cap. 13, seç. 22]), a restrição do Teorema do Produto de Tychonoff aos espaços topológicos compactos e de Hausdorff e o Teorema da Compactificação de Stone-Čech (v. [Sch1997, cap. 17, seç. 22]). Um fato pouco citado é que, em ZF, o Teorema do Ultrafiltro é equivalente ao Teorema de Banach-Alaoglu (v. [JF2018, seç. 3 e 5]). No que segue, é apresentado o enunciado mais conhecido de tal princípio maximal juntamente com a sigla que irá representá-lo nesta tese:

Teorema do Ultrafiltro (UT). Para todo conjunto não vazio I e todo filtro $\mathcal{F}$ sobre I, existe um ultrafiltro $\mathcal{U}$ sobre I tal que $\mathcal{F} \subseteq \mathcal{U}$.

A versão original de UT foi apresentada por A. Tarski em [Tar1930], onde se prova tal versão utilizando AC. ${ }^{1}$ Com o uso dos itens (i), (ii) (1) e (iv) (1) do Comentário 1.1.3 e dos itens (ii) e (iii) do Comentário 1.1.2, conclui-se o seguinte

Teorema 1.1.6. Em ZF, são equivalentes:

(i) $\mathbf{U T}$.

(ii) Para todo conjunto não vazio I e todo $\mathcal{A} \subseteq \mathcal{P}(I)$, se $\mathcal{A}$ tiver a PIF, então existe um ultrafiltro $\mathcal{U}$ sobre I tal que $\mathcal{A} \subseteq \mathcal{U}$.

${ }^{1}$ É interessante destacar que $\mathbf{A C}_{\omega}$ não implica $\mathbf{U T}$ em $\mathbf{Z F}$, já que, no chamado primeiro modelo de Pincus-Solovay (denotado por $\mathcal{M} 27$ em [HR1998]), AC $\omega$ é verdadeiro e UT é falso (i.e., $\mathcal{M} 27$ é um modelo de $\left.\mathbf{Z F}+\mathbf{A C}_{\omega}+\neg \mathbf{U T}\right)$. Além disso, sabe-se que $\mathbf{U T}$ não implica $\mathbf{A} \mathbf{C}_{\omega} \mathrm{em} \mathbf{Z F}$, pois, no chamado primeiro modelo de Cohen (denotado por $\mathcal{M} 1$ em [HR1998]), UT é verdadeiro e $\mathbf{A C}_{\omega}$ é falso (i.e., $\mathcal{M} 1$ é um modelo de $\left.\mathbf{Z F}+\mathbf{U T}+\neg \mathbf{A C} \mathbf{C}_{\omega}\right)$. Logo, $\mathbf{A} \mathbf{C}_{\omega}$ e $\mathbf{U T}$ são princípios mais fracos que $\mathbf{A C}$ em $\mathbf{Z F}$. 
Definição 1.1.7. Sejam $X$ um conjunto e $R$ uma relação binária sobre $X$. Considere as seguintes condições:

(a) (reflexividade) Para todo $x \in X, x R x$.

(b) (simetria) Para quaisquer $x, y \in X$, se $x R y$, então $y R x$.

(c) (antissimetria) Para quaisquer $x, y \in X$, se $x R y$ e $y R x$, então $x=y$.

(d) (conexidade) Para quaisquer $x, y \in X, x=y$ ou $x R$ ou $y R x$.

(e) (transitividade) Para quaisquer $x, y, z \in X$, se $x R$ y e $y R z$, então $x R z$.

( $f$ ) (propriedade de Moore-Smith) Para quaisquer $x, y \in X$, existe um $z \in X$ tal que $x R z$ e $y R z$.

Diremos que:

(i) $R$ é uma pré-ordem sobre $X$ se valerem as condições $(a)$ e $(e)$. Se $R$ for uma pré-ordem sobre $X$, diremos que o par $\langle X, R\rangle$ é um conjunto pré-ordenado.

(ii) $R$ é uma relação de equivalência sobre $X$ se $R$ for uma pré-ordem sobre $X$ e valer a condição $(b)$. Se $R$ for uma relação de equivalência sobre $X$, diremos que o par $\langle X, R\rangle$ é um conjuntoide.

(iii) $R$ é uma pré-ordem total sobre $X$ se $R$ for uma pré-ordem sobre $X$ e valer a condição $(d)$. Se $R$ for uma pré-ordem total sobre $X$, diremos que o par $\langle X, R\rangle$ é um conjunto totalmente pré-ordenado.

(iv) $R$ é uma direção sobre $X$ se $R$ for uma pré-ordem sobre $X$ e valer a condição $(f)$. Se $R$ for uma direção sobre $X$, diremos que o par $\langle X, R\rangle$ é um conjunto dirigido.

(v) R é uma ordem parcial sobre $X$ se $R$ for uma pré-ordem sobre $X$ e valer a condição (c). Se $R$ for uma ordem parcial sobre $X$, diremos que o par $\langle X, R\rangle$ é um conjunto parcialmente ordenado.

(vi) $R$ é uma ordem total sobre $X$ se $R$ for uma ordem parcial sobre $X$ e valer a condição $(d)$. Se $R$ for uma ordem total sobre $X$, diremos que o par $\langle X, R\rangle$ é um conjunto totalmente ordenado. 
A relação binária $R \backslash \operatorname{id}_{X}$ será chamada de a redução reflexiva de $R$ e denotada por $R^{\neq}$. Suponha que $R$ seja uma pré-ordem sobre $X$. Para qualquer $x \in X$,

(1) o conjunto $\left\{z \in X: x R^{\neq} z\right\}$ será chamado de o intervalo aberto segundo $R$ de extremo inferior $x$ e denotado por $] x, \rightarrow\left[{ }_{R}\right.$.

(2) o conjunto $\left\{z \in X: z R^{\neq} x\right\}$ será chamado de o intervalo aberto segundo $R$ de extremo superior $x$ e denotado por $] \leftarrow, x\left[{ }_{R}\right.$.

(3) o conjunto $\{x\} \cup] x, \rightarrow\left[_{R}\right.$ será chamado de o intervalo fechado segundo $R$ de extremo inferior $x$ e denotado por $\left[x, \rightarrow\left[{ }_{R}\right.\right.$.

(4) o conjunto $\{x\} \cup] \leftarrow, x\left[_{R}\right.$ será chamado de o intervalo fechado segundo $R$ de extremo superior $x$ e denotado por $] \leftarrow, x]_{R}$.

Para quaisquer $x, y \in X$, se $x R y$, então

(5) o conjunto $\left\{z \in X: x R^{\neq} z \wedge z R^{\neq} y\right\}$ será chamado de o intervalo aberto segundo $R$ de extremos $x$ e $y$ e denotado por $] x, y\left[_{R}\right.$.

(6) o conjunto $\{y\} \cup] x, y\left[_{R}\right.$ será chamado de o intervalo semiaberto à esquerda segundo $R$ de extremos $x$ e $y$ e denotado por $] x, y]_{R}$.

(7) o conjunto $\{x\} \cup] x, y\left[_{R}\right.$ será chamado de o intervalo semiaberto à direita segundo $R$ de extremos $x$ e $y$ e denotado por $\left[x, y\left[_{R}\right.\right.$.

(8) o conjunto $\{x, y\} \cup] x, y\left[_{R}\right.$ será chamado de o intervalo fechado segundo $R$ de extremos $x$ e $y$ e denotado por $[x, y]_{R}$.

Suponha que $R$ seja uma relação de equivalência sobre $X$. Para qualquer $x \in X$,

(9) o intervalo $] \leftarrow, x]_{R}$ será chamado de a classe de equivalência de $x$ módulo $R$ e denotado por $[x]_{R}$.

A família $\left\{[x]_{R}: x \in X\right\}$ será chamada de o conjunto quociente de $X$ por $R$ e denotada por $X / R$.

Comentário 1.1.8. Dados um conjunto pré-ordenado $\langle X, R\rangle$ e um $A \subseteq X$, será dito que $A$ é um denso segundo $R$ se valer a seguinte condição: para quaisquer $x, y \in X$, se $x R^{\neq} y$, então o conjunto $] x, y\left[_{R} \cap A\right.$ é não vazio. É imediato concluir que:

(i) $X$ é um denso segundo $R$ se, e somente se, para quaisquer $x, y \in X$, se $x R^{\neq} y$, então o intervalo $] x, y\left[_{R}\right.$ é não vazio. 
Considere a relação binária $R_{A}$ sobre $X$ que é definida pela seguinte sentença:

$$
\text { para quaisquer } x, y \in X, x R_{A} y \text { se, e só se, } x R y \text {. }
$$

A relação binária $R_{A}$ será chamada de a relação induzida por $R$ sobre $A$. É fácil ver que:

(ii) (1) Se $R$ for uma pré-ordem sobre $X$, então $R_{A}$ é uma pré-ordem sobre $A$.

(2) Se $R$ for uma relação de equivalência sobre $X$, então $R_{A}$ é uma relação de equivalência sobre $A$.

(3) Se $R$ for uma pré-ordem total sobre $X$, então $R_{A}$ é uma pré-ordem total sobre $A$.

(4) Se $R$ for uma ordem parcial sobre $X$, então $R_{A}$ é uma ordem parcial sobre $A$.

(5) Se $R$ for uma ordem total sobre $X$, então $R_{A}$ é uma ordem total sobre $A$.

Agora, suponha que $R$ seja uma relação de equivalência sobre $X$. Considere a função $q_{R}: X \rightarrow X / R$ que é definida pondo-se, para quaisquer $x, y \in X$,

$$
q_{R}(x):=[x]_{R}
$$

A função $q_{R}$ será chamada de a função quociente para $\langle X, R\rangle$. Note que:

(iii) (1) $q_{R}$ é sobrejetora.

(2) $q_{R}$ é bijetora se, e somente se, $R=\mathrm{id}_{X}$.

Definição 1.1.9. Sejam $X$ um conjunto, $\mathcal{N} \subseteq \mathcal{P}(X), \tau$ uma topologia sobre $X$ e $a \in X$. Denote por $\mathcal{N} \cup$ a família $\{\cup \mathcal{A}: \mathcal{A} \in \mathcal{P}(\mathcal{N})\}$. Diremos que:

(i) $\mathcal{N}$ gera uma topologia sobre $X$ se $\mathcal{N} \cup$ for uma topologia sobre $X$. Se $\mathcal{N}$ gerar uma topologia sobre $X$, diremos que $\mathcal{N} \cup$ é a topologia gerada por $\mathcal{N}$ (sobre $X$ ).

(ii) $\mathcal{N}$ é uma network para $\tau$ se $\tau=\mathcal{N} \cup$.

(iii) $\mathcal{N}$ é uma base para $\tau$ se valerem as seguintes condições:

(1) $\mathcal{N} \subseteq \tau$.

(2) $\mathcal{N}$ é uma network para $\tau$.

(iv) $\mathcal{N}$ é uma network local para $\tau$ em $a$ se $\mathcal{N}_{\tau}(a) \cap \tau=\mathcal{N} \cup$.

(v) $\mathcal{N}$ é uma base local para $\tau$ em $a$ se valerem as seguintes condições: 
(1) $\mathcal{N} \subseteq \tau$.

(2) $\mathcal{N}$ é uma network local para $\tau$ em $a$.

(vi) $\langle X, \tau\rangle$ é zero-dimensional se valer a seguinte condição: existe uma base $\mathcal{B}$ para $\tau$ tal que, para todo $B \in \mathcal{B}, B$ é um fechado em $\langle X, \tau\rangle .^{2}$

(vii) $\langle X, \tau\rangle$ tem peso enumerável se valer a seguinte condição: existe uma base $\mathcal{B}$ para $\tau$ tal que $\mathcal{B}$ é enumerável.

(viii) $\langle X, \tau\rangle$ tem caráter enumerável se valer a seguinte condição: para todo $x \in X$, existe uma base local $\mathcal{B}$ para $\tau$ em $x$ tal que $\mathcal{B}$ é enumerável.

Comentário 1.1.10. Dados um conjunto $X$ e um $\mathcal{N} \subseteq \mathcal{P}(X)$, note que:

(i) (1) $\emptyset \in \mathcal{N} \cup$.

(2) $X \in \mathcal{N} \cup$ se, e somente se, $\mathcal{N}$ for uma cobertura de $X$.

(3) (1) $\mathcal{N} \subseteq \mathcal{N} \cup$.

(2) $\mathcal{N}^{\cup} \subseteq \bigcap\{\mathcal{A} \in \mathcal{P}(\mathcal{P}(X)): \mathcal{N} \subseteq \mathcal{A} \wedge \mathcal{A}$ é uma topologia sobre $X\}$.

Mostra-se facilmente que:

(ii) São equivalentes:

(1) $\mathcal{N}$ gera uma topologia sobre $X$.

(2) $\mathcal{N} \cup=\bigcap\{\mathcal{A} \in \mathcal{P}(\mathcal{P}(X)): \mathcal{N} \subseteq \mathcal{A} \wedge \mathcal{A}$ é uma topologia sobre $X\}$.

(3) Valem as seguintes condições:

(1) $\mathcal{N}$ é uma cobertura de $X$.

(2) Para todo $x \in X$ e quaisquer $A, B \in \mathcal{N}$, se $x \in A \cap B$, então existe um $C \in \mathcal{N}$ tal que $x \in C$ e $C \subseteq A \cap B$.

Dada uma topologia $\tau$ sobre $X$, suponha que $\mathcal{N}$ seja uma base para $\tau$. É fácil verificar que:

(iii) Para todo $A \subseteq X$,

\footnotetext{
${ }^{2} \mathrm{O}$ termo zero-dimensional refere-se à dimensão de Menger-Urysohn e a sua definição é justificada pela asserção

para todo espaço topológico $\langle X, \tau\rangle$ tal que $X$ é não vazio, existe uma base $\mathcal{B}$ para $\tau$ tal que todo membro de $\mathcal{B}$

é um fechado em $\langle X, \tau\rangle$ se, e somente se, a dimensão de Menger-Urysohn de $\langle X, \tau\rangle$ for nula, que se encontra, por exemplo, em [Eng1989, cap. 7, seç. 1].
} 
(1) $\bar{A}^{\tau}=\left\{x \in X: \forall B \in \mathcal{N}_{\tau}(x) \cap \mathcal{N}(B \cap A \neq \emptyset)\right\}$.

(2) $A$ é um denso em $\langle X, \tau\rangle$ se, e somente se, para todo $B \in \mathcal{N} \backslash\{\emptyset\}, B \cap A \neq \emptyset$.

Dados um espaço topológico $\left\langle X^{\prime}, \tau^{\prime}\right\rangle$, uma base $\mathcal{N}^{\prime}$ para $\tau^{\prime}$ e uma função $\varphi: X \rightarrow X^{\prime}$, também é fácil verificar que:

(iv) (1) $\varphi$ é contínua com respeito a $\tau$ e $\tau^{\prime}$ se, e somente se, para todo $B \in \mathcal{N}^{\prime}$, $\varphi^{-1}[B] \in \tau$.

(2) $\varphi$ é aberta com respeito a $\tau$ e $\tau^{\prime}$ se, e somente se, para todo $A \in \mathcal{N}, \varphi[A] \in \tau^{\prime}$. Agora, considere a seguinte família:

$$
\mathcal{B}\left(\tau, \tau^{\prime}\right):=\left\{V \times V^{\prime}: V \in \tau \wedge V^{\prime} \in \tau^{\prime}\right\} .
$$

Usando o item ( $\mathrm{i}$ ), é corriqueiro mostrar que:

(v) $\mathcal{B}\left(\tau, \tau^{\prime}\right)$ gera uma topologia sobre $X \times X^{\prime}$.

A topologia gerada $\left(\mathcal{B}\left(\tau, \tau^{\prime}\right)\right)^{\cup}$ será chamada de a topologia produto de $\tau$ e $\tau^{\prime}$ (sobre $X \times X^{\prime}$ ) e denotada por $\tau \otimes \tau^{\prime}$. O espaço topológico $\left\langle X \times X^{\prime}, \tau \otimes \tau^{\prime}\right\rangle$ será chamado de o produto topológico de $\langle X, \tau\rangle$ e $\left\langle X^{\prime}, \tau^{\prime}\right\rangle$ e denotado por $\langle X, \tau\rangle \otimes\left\langle X^{\prime}, \tau^{\prime}\right\rangle$. Dados um $\alpha \in X \times X^{\prime}$ e um $V \in \mathcal{N}_{\tau \otimes \tau^{\prime}}(\alpha) \cap \mathcal{B}\left(\tau, \tau^{\prime}\right)$, será dito que $V$ é uma vizinhança canônica de $\alpha \operatorname{em}\langle X, \tau\rangle \otimes\left\langle X^{\prime}, \tau^{\prime}\right\rangle$.

Proposição 1.1.11. Seja $\langle X, \tau\rangle$ um espaço topológico. Então, são equivalentes:

(i) $\langle X, \tau\rangle$ é compacto.

(ii) Para todo $\mathcal{A} \subseteq \mathcal{P}(X)$, se $\mathcal{A}$ for não vazio e tiver a PIF e todo membro de $\mathcal{A}$ for um fechado em $\langle X, \tau\rangle$, então o conjunto $\bigcap \mathcal{A}$ é não vazio.

(iii) Existe uma base $\mathcal{B}$ para $\tau$ tal que, para todo $\mathcal{C} \subseteq \mathcal{B}$, se $C$ for uma cobertura de $X$, então existe um subfamília finita de $C$ que é uma cobertura de $X$.

Comentário 1.1.12. Dados conjuntos $X$ e $X^{\prime}$, uma função $\varphi: X \rightarrow X^{\prime}$ e um $A \subseteq X$, considere a função $\varphi\left\lceil A: A \rightarrow X^{\prime}\right.$ que é definida pondo-se, para todo $x \in A$,

$$
(\varphi \uparrow A)(x):=\varphi(x) \text {. }
$$

A função $\varphi\left\lceil A\right.$ será chamada de a restrição de $\varphi$ a $A$. Dado um $A^{\prime} \subseteq X^{\prime}$ tal que $\operatorname{im}(\varphi) \subseteq A^{\prime}$, também considere a função $\varphi \downarrow A^{\prime}: X \rightarrow A^{\prime}$ que é definida pondo-se, para todo $x \in X$,

$$
\left(\varphi \downarrow A^{\prime}\right)(x):=\varphi(x) .
$$

A função $\varphi\left\lfloor A^{\prime}\right.$ será chamada de a correstrição de $\varphi$ a $A^{\prime}$. É claro que: 
(i) (1) Se $\varphi$ for injetora, então $\varphi \uparrow A$ é injetora.

(2) $\varphi \downarrow A^{\prime}$ é injetora se, e somente se, $\varphi$ for injetora.

(ii) (1) (1) $\operatorname{im}(\varphi \uparrow A)=\varphi[A]$.

(2) $\varphi \uparrow A$ é sobrejetora se, e somente se, $\varphi[A]=X^{\prime}$.

(2) (1) $\operatorname{im}\left(\varphi\left\lfloor A^{\prime}\right)=\operatorname{im}(\varphi)\right.$.

(2) $\varphi l A^{\prime}$ é sobrejetora se, e somente se, $\operatorname{im}(\varphi)=A^{\prime}$.

É fácil verificar que:

(iii) (1) Para todo $B \subseteq A,(\varphi \uparrow A)[B]=\varphi[B]$.

(2) Para todo $B \subseteq X$,

(1) $\left(\varphi\left\lfloor A^{\prime}\right)[B]=\varphi[B]\right.$.

(2) $\left(\varphi \downarrow A^{\prime}\right)[B] \subseteq A^{\prime}$.

(3) Para todo $B \subseteq X^{\prime}$,

(1) $\left(\varphi\lceil A)^{-1}[B]=\varphi^{-1}[B] \cap A\right.$.

(2) $\left(\varphi\left\lfloor A^{\prime}\right)^{-1}\left[B \cap A^{\prime}\right]=\varphi^{-1}[B]\right.$.

(iv) (1) Se $\varphi$ for injetora e $\varphi[A]=X^{\prime}$, então $\varphi \uparrow A$ é bijetora.

(2) $\varphi\left\lfloor A^{\prime}\right.$ é bijetora se, e somente se, $\varphi$ for injetora e $\operatorname{im}(\varphi)=A^{\prime}$.

(3) (1) Se $\varphi$ for injetora, então $(\varphi \uparrow A) \downarrow \varphi[A]$ é bijetora.

(2) Se $\varphi$ for bijetora, então $\left((\varphi\lceil A) \downarrow \varphi[A])^{-1}=\left(\left(\varphi^{-1}\right) \uparrow \varphi[A]\right) \downarrow A\right.$.

Dada uma topologia $\tau$ sobre $X$, mostra-se facilmente que:

(v) A família $\{V \cap A: V \in \tau\}$ é uma topologia sobre $A$.

Esta família será chamada de a topologia de subespaço de $\langle X, \tau\rangle$ sobre $A$ e denotada por $\tau_{A}$. É fácil verificar que:

(vi) (1) Para todo $B \subseteq A,\left(\tau_{A}\right)_{B}=\tau_{B}$.

(2) Para toda base $\mathcal{B}$ para $\tau$, a família $\{B \cap A: B \in \mathcal{B}\}$ é uma base para $\tau_{A}$.

É corriqueiro mostrar que:

(vii) Para todo $B \subseteq A$,

(1) $B$ é um fechado em $\left\langle A, \tau_{A}\right\rangle$ se, e somente se, existir um $C \subseteq X$ tal que $C$ é um fechado em $\langle X, \tau\rangle$ e $B=C \cap A$. 
(2) $\bar{B}^{\tau_{A}}=\bar{B}^{\tau} \cap A$.

Dada uma topologia $\tau^{\prime}$ sobre $X^{\prime}$, usando os itens (iii) (3) e (iii) (2), verifica-se facilmente que:

(viii) (1) Se $\varphi$ for contínua com respeito a $\tau$ e $\tau^{\prime}$, então $\varphi \uparrow A$ é contínua com respeito a $\tau_{A}$ e $\tau^{\prime}$.

(2) (1) $\varphi \downarrow A^{\prime}$ é contínua com respeito a $\tau$ e $\left(\tau^{\prime}\right)_{A^{\prime}}$ se, e somente se, $\varphi$ for contínua com respeito a $\tau$ e $\tau^{\prime}$.

(2) Se $\varphi$ for aberta com respeito a $\tau$ e $\tau^{\prime}$, então $\varphi \downarrow A^{\prime}$ é aberta com respeito a $\tau$ e $\left(\tau^{\prime}\right)_{A^{\prime}}$.

Usando os itens (viii) (1), (viii) (2) (1) e (iv) (2) (1), tem-se que:

(ix) (1) Se $\varphi$ for contínua com respeito a $\tau$ e $\tau^{\prime}$, então $(\varphi\lceil A) \downarrow \varphi[A]$ é contínua com respeito a $\tau_{A}$ e $\left(\tau^{\prime}\right)_{\varphi[A]}$.

(2) Se $\varphi$ for um homeomorfismo com respeito a $\tau$ e $\tau^{\prime}$, então $(\varphi \uparrow A) \downarrow \varphi[A]$ é um homeomorfismo com respeito a $\tau_{A}$ e $\left(\tau^{\prime}\right)_{\varphi[A]}$.

Se $\varphi \lim (\varphi)$ for um homeomorfismo com respeito a $\tau$ e $\left(\tau^{\prime}\right)_{\operatorname{im}(\varphi)}$, será dito que $\varphi$ é uma imersão topológica com respeito a $\tau$ e $\tau^{\prime}$. Usando os itens (i) (2), (viii)(2)(1), (viii) (2) (2) e (ii) (2) (2), conclui-se que:

(x) (1) Se $\varphi$ for uma imersão topológica com respeito a $\tau$ e $\tau^{\prime}$, então $\varphi$ é uma injeção que é contínua com respeito a $\tau$ e $\tau^{\prime}$.

(2) Se $\varphi$ for uma injeção que é contínua e aberta com respeito a $\tau$ e $\tau^{\prime}$, então $\varphi$ é uma imersão topológica com respeito a $\tau$ e $\tau^{\prime}$.

Proposição 1.1.13. Sejam $\langle X, \tau\rangle$ um espaço topológico e $A \subseteq X$. Então:

(i) Se $\langle X, \tau\rangle$ for compacto e A for um fechado em $\langle X, \tau\rangle$, então $\left\langle A, \tau_{A}\right\rangle$ é compacto.

(ii) Para todo $B \subseteq X$, se $A \subseteq B$ e $\left\langle A,\left(\tau_{B}\right)_{A}\right\rangle$ for compacto, então $\left\langle A, \tau_{A}\right\rangle$ é compacto.

Definição 1.1.14. Sejam $\mathbb{K}$ um corpo e $\sigma$ uma topologia sobre o conjunto $\mathbb{K}$. Diremos que o par $\langle\mathbb{K}, \sigma\rangle$ é um corpo topológico se valerem as seguintes condições:

(i) A adição sobre $\mathbb{K}$ é contínua com respeito a $\sigma \otimes \sigma$ e $\sigma$. 
(ii) A multiplicação sobre $\mathbb{K}$ é contínua com respeito a $\sigma \otimes \sigma$ e $\sigma$.

(iii) A inversão em $\mathbb{K} \backslash\{0\}$ é contínua com respeito a $\sigma_{\mathbb{K} \backslash\{0\}}$ e $\sigma_{\mathbb{K} \backslash\{0\}}$.

Comentário 1.1.15. Dado um conjunto pré-ordenado $\langle X, \leqslant\rangle$, denotaremos por $<$ a redução reflexiva $\leqslant^{\neq}$. Considere a seguinte família:

$$
\mathcal{B}_{\leqslant}:=\{X\} \cup\{] \leftarrow, x\left[_{\leqslant}: x \in X\right\} \cup\{] x, \rightarrow\left[_{\leqslant}: x \in X\right\} \cup\{] x, y\left[_{\leqslant}: x, y \in X \wedge x<y\right\} .
$$

Suponha que $\leqslant$ seja uma ordem total sobre X. Usando o Comentário 1.1.10(ii), é fácil concluir que:

(i) $\mathcal{B}_{\leqslant}$gera uma topologia sobre $X$.

A topologia gerada $\left(\mathcal{B}_{\leqslant}\right)^{\cup}$ será chamada de a topologia da ordem sobre $X$ segundo $\leqslant \mathrm{e}$ denotada por $\tau_{\leqslant}$. É fácil concluir que:

(ii) Para quaisquer $x, y \in X$, se $x \leqslant y$, então

(1) $\left.X \backslash[x, y]_{\leqslant}=\right] \leftarrow, x[\leqslant \cup] y, \rightarrow\left[_{\leqslant}\right.$.

(2) $[x, y]_{\leqslant}$é um fechado em $\left\langle X, \tau_{\leqslant}\right\rangle$.

É corriqueiro verificar que:

(iii) (1) Para quaisquer $x, y \in X$, se $x<y$, então existem $z$, w $\in X$ tais que $x<w$, $z<y \mathrm{e}] \leftarrow, w[\leqslant \cap] z \rightarrow[\leqslant=\emptyset$.

(2) $\left\langle X, \tau_{\leqslant}\right\rangle$é de Hausdorff. ${ }^{3}$

É fácil ver que:

(iv) Para todo $A \subseteq X$, se $A$ for um denso segundo $\leqslant$, então $A$ é um denso em $\left\langle X, \tau_{\leqslant}\right\rangle$.

Dado um ordinal $\left\langle\alpha,<_{\alpha}\right\rangle$, denote por $\leqslant_{\alpha}$ a ordem total sobre $\alpha$ tal que $\left(\leqslant_{\alpha}\right)^{\neq}=<_{\alpha}$. É fácil verificar que:

(v) Se $\alpha<\omega+1$, então $\tau_{\leqslant \alpha}=\mathcal{P}(\alpha)$.

\footnotetext{
${ }^{3}$ É interessante destacar que, em ZFC, prova-se que tal espaço topológico é hereditariamente normal (v. [Ste1970] e [SS1978, p. 66-67]). No entanto, em ZF não é possível provar a asserção para todo conjunto totalmente ordenado $\langle X, \leqslant\rangle,\left\langle X, \tau_{\leqslant}\right\rangle$é hereditariamente normal,
} já que esta última é falsa em pelo menos um modelo de ZF $+\neg$ AC (v. [Kro1986, VD1985]). 
É claro que:

(vi) Se $1<\alpha$, então $\alpha$ é um denso em $\left\langle\alpha, \tau_{\leqslant_{\alpha}}\right\rangle$ que não é um denso segundo $\leqslant \alpha$.

Agora, denote por $\leqslant$ a ordem total do corpo ordenado $\mathbb{R}$. Afirmamos que:

(viii) A família \{]$\lambda-\varepsilon, \lambda+\varepsilon\left[: \lambda \in \mathbb{R} \wedge \varepsilon \in \mathbb{R}^{+}\right\}$é uma base para $\tau_{\leqslant}$.

De fato: denote por $\mathcal{I}$ a família \{]$\lambda-\varepsilon, \lambda+\varepsilon\left[: \lambda \in \mathbb{R} \wedge \varepsilon \in \mathbb{R}^{+}\right\}$. É claro que $\mathcal{I} \cup \subseteq \tau_{\leqslant}$. Fixe arbitrariamente $\lambda, v \in \mathbb{R}$. Note que:

- se $\lambda<v$, então, tomando-se $\delta:=\frac{1}{2}(v-\lambda)$, tem-se que:

$$
\left.\delta \in \mathbb{R}^{+} \mathrm{e}\right] \lambda, v[=] \frac{1}{2}(\lambda+v)-\delta, \frac{1}{2}(\lambda+v)+\delta[.
$$

- utilizando-se da propriedade arquimediana do corpo ordenado $\mathbb{R}$, tem-se que:

$$
\begin{aligned}
]-\infty, \lambda[ & \left.=\bigcup_{n \in \omega \backslash 1}\right] \lambda-n, \lambda[, \\
] \lambda,+\infty[ & \left.=\bigcup_{n \in \omega \backslash 1}\right] \lambda, \lambda+n[ \\
& \mathrm{e} \\
\mathbb{R} & \left.=\bigcup_{n \in \omega \backslash 1}\right]-n, n[.
\end{aligned}
$$

Logo, $\tau_{\leqslant} \subseteq \mathcal{I} \cup$.

Denote por $|\cdot|$ a função valor absoluto dos números reais (i.e., a função de $\mathbb{R}$ em $\mathbb{R}$ que é definida pondo-se, para todo $\lambda \in \mathbb{R}$,

$$
|\lambda|:=\leqslant-\max \{-\lambda, \lambda\}=\left\{\begin{aligned}
\lambda, & \text { se } 0 \leqslant \lambda \\
-\lambda, & \text { caso contrário). }
\end{aligned}\right.
$$

Afirmamos que:

(ix) $|\cdot|$ é contínua com respeito a $\tau_{\leqslant} \mathrm{e} \tau_{\leqslant}$.

(x) O par $\left\langle\mathbb{R}, \tau_{\leqslant}\right\rangle$é um corpo topológico.

De fato: fixe arbitrariamente $\lambda, v \in \mathbb{R}$ e um $\varepsilon \in \mathbb{R}^{+}$.

$(i x)$ e $(x)$ : Note que:

- Tomando-se $\delta:=\varepsilon$, tem-se que: 
$\delta \in \mathbb{R}^{+}$e, para todo $x \in \mathbb{R}$, se $\left.x \in\right] \lambda-\delta, \lambda+\delta[$, então $|x| \in]|\lambda|-\varepsilon,|\lambda|+\varepsilon[$.

- Tomando-se $\delta:=\frac{1}{2} \varepsilon$, tem-se que:

$\delta \in \mathbb{R}^{+}$e, para quaisquer $x, y \in \mathbb{R}$, se $\left.\langle x, y\rangle \in\right] \lambda-\delta, \lambda+\delta[\times] v-\delta, v+\delta[$, então $x+y \in](\lambda+v)-\varepsilon,(\lambda+v)+\varepsilon[$.

- Tomando-se $\delta:=\min \left\{1, \frac{1}{3} \varepsilon(1+|\lambda|)^{-1}, \frac{1}{3} \varepsilon(1+|v|)^{-1}\right\}$, tem-se que:

$\delta \in \mathbb{R}^{+}$e, para quaisquer $x, y \in \mathbb{R}$, se $\left.\langle x, y\rangle \in\right] \lambda-\delta, \lambda+\delta[\times] v-\delta, v+\delta[$, então $x y \in] \lambda v-\varepsilon, \lambda v+\varepsilon[$.

- Tomando-se $\delta:=\frac{1}{2} \min \left\{|\lambda|, \varepsilon \lambda^{2}\right\}$, tem-se que:

$$
\begin{aligned}
& \left.\delta \in \mathbb{R}^{+} \text {e, para todo } x \in \mathbb{R} \backslash\{0\}, \text { se } x \in\right] \lambda-\delta, \lambda+\delta[\text {, então } \\
& \left.x^{-1} \in\right] \lambda^{-1}-\varepsilon, \lambda^{-1}+\varepsilon[.
\end{aligned}
$$

Em virtude do item (viii) e dos Comentários 1.1.10(iv) (1) e 1.1.12(vi) (2), conclui-se que: $|\cdot|$ é contínua com respeito a $\tau_{\leqslant}$e $\tau_{\leqslant}$, a adição e a multiplicação sobre $\mathbb{R}$ são contínuas com respeito a $\tau_{\leqslant} \otimes \tau_{\leqslant} \mathrm{e} \tau_{\leqslant}$e a inversão em $\mathbb{R} \backslash\{0\}$ é contínua com respeito $\mathrm{a}\left(\tau_{\leqslant}\right)_{\mathbb{R} \backslash\{0\}}$ e $\left(\tau_{\leqslant}\right)_{\mathbb{R} \backslash\{0\}}$.

A topologia $\tau_{\leqslant}$será chamada de a topologia usual sobre $\mathbb{R}$ e denotada por $\tau_{2}$.

Proposição 1.1.16. Sejam $\langle X, \tau\rangle$ um espaço topológico e $A \subseteq X$. Então:

(i) Se $\langle X, \tau\rangle$ for $T_{0}$, então $\left\langle A, \tau_{A}\right\rangle$ é $T_{0}$.

(ii) Supondo-se que $\langle X, \tau\rangle$ seja $T_{1}$, tem-se que:

(1) $\langle X, \tau\rangle$ é $T_{0}$.

(2) $\left\langle A, \tau_{A}\right\rangle$ é $T_{1}$.

(iii) Supondo-se que $\langle X, \tau\rangle$ seja de Hausdorff, tem-se que:

(1) $\langle X, \tau\rangle$ é $T_{1}$.

(2) $\left\langle A, \tau_{A}\right\rangle$ é de Hausdorff.

(iv) Supondo-se que $\langle X, \tau\rangle$ seja regular, tem-se que:

(1) $\langle X, \tau\rangle$ é de Hausdorff. 
(2) $\left\langle A, \tau_{A}\right\rangle$ é regular.

(v) Supondo-se que $\langle X, \tau\rangle$ seja de Tychonoff, tem-se que:

(1) $\langle X, \tau\rangle$ é regular.

(2) $\left\langle A, \tau_{A}\right\rangle$ é de Tychonoff.

Proposição 1.1.17. Sejam X um conjunto, $\tau$ uma topologia sobre $X$ e $A \subseteq X$. Então:

(i) (1) $(\mathcal{P}(X))_{A}=\mathcal{P}(A)$.

(2) $\langle X, \mathcal{P}(X)\rangle$ é $T_{1}$.

(3) $\langle X, \mathcal{P}(X)\rangle$ é de Tychonoff.

(4) $\langle X, \mathcal{P}(X)\rangle$ é zero-dimensional.

(5) $\langle X, \mathcal{P}(X)\rangle$ é compacto se, e somente se, $X$ for finito.

(ii) Supondo-se que $\langle X, \tau\rangle$ seja zero-dimensional, tem-se que:

(1) $\left\langle A, \tau_{A}\right\rangle$ é zero-dimensional.

(2) São equivalentes:

(1) $\langle X, \tau\rangle$ é de Tychonoff.

(2) $\langle X, \tau\rangle$ é regular.

(3) $\langle X, \tau\rangle$ é de Hausdorff.

(4) $\langle X, \tau\rangle$ é $T_{1}$.

(5) $\langle X, \tau\rangle$ é $T_{0}$.

Proposição 1.1.18. Dados um espaço topológico $\langle X, \tau\rangle$ tal que $X$ é não vazio, uma sequência s em $X, u m A \subseteq X$ tal que $\operatorname{im}(s) \subseteq A$ e um $a \in A$, considere as seguintes asserções:

(a) A sequência s converge para a com respeito a $\tau$.

(b) A sequência s converge para a com respeito a $\tau_{A}$.

(c) A sequência sé constante de valor a.

(d) A sequência s é eventualmente constante de valor a.

Então: 
(i) (a) é equivalente a (b).

(ii) (c) implica (d).

(iii) (d) implica (a).

Suponha que a seja um ponto isolado de $\langle X, \tau\rangle$. Então:

(iv) (a) é equivalente a (d).

Proposição 1.1.19. Dados um espaço topológico $\langle X, \tau\rangle$ tal que X é não vazio, uma sequência s em $X$, um $A \subseteq X$ tal que $\operatorname{im}(s) \subseteq A$ e um $a \in X$, considere as seguintes asserções:

(a) A sequência s converge para a com respeito a $\tau$.

(b) Para toda subsequência $t$ de $s, t$ converge para a com respeito a $\tau$.

(c) $a \in \bar{A}^{\tau}$.

Então:

(i) (a) é equivalente a (b).

(ii) (a) implica (c).

Teorema 1.1.20. Dado um espaço topológico $\langle X, \tau\rangle$ tal que X é não vazio, considere as seguintes asserções:

(a) $\langle X, \tau\rangle$ é de Hausdorff.

(b) Para toda sequência s em $X$, se s for convergente com respeito a $\tau$, então existe um único limite de s com respeito a $\tau$.

Então:

(i) (a) implica (b).

Suponha que $\langle X, \tau\rangle$ tenha caráter enumerável. Então:

(ii) Sob $\mathbf{A C}_{\omega}$, (a) é equivalente a (b). 
Proposição 1.1.21. Sejam $\langle X, \tau\rangle$ e $\left\langle X^{\prime}, \tau^{\prime}\right\rangle$ espaços topológicose $\left\langle X^{\prime \prime}, \tau^{\prime \prime}\right\rangle$ um espaço topológico de Hausdorff. Sejam $\gamma: X \rightarrow X^{\prime}$ uma função contínua com respeito a $\tau$ e $\tau^{\prime}$ e $\psi, \psi^{\prime}: X^{\prime} \rightarrow X^{\prime \prime}$ funções contínuas com respeito a $\tau^{\prime}$ e $\tau^{\prime \prime}$. Então:

(i) O conjunto $\left\{z \in X^{\prime}: \psi(z)=\psi^{\prime}(z)\right\}$ é um fechado em $\left\langle X^{\prime}, \tau^{\prime}\right\rangle$.

(ii) Se o conjunto $\operatorname{im}(\gamma)$ for um denso em $\left\langle X^{\prime}, \tau^{\prime}\right\rangle$ e $\psi \circ \gamma=\psi^{\prime} \circ \gamma$, então $\psi=\psi^{\prime}$

Encerraremos esta seção com a apresentação das definições de soma e de produto topológicos e de resultados já conhecidos sobre esses espaços topológicos.

Comentário 1.1.22 ([Eng1989, cap. 2, seç. 2]). Dados um conjunto $I$ e uma família $\left\{X_{i}: i \in I\right\}$ de conjuntos, denotaremos por $\sum_{i \in I} X_{i}$ (ou por $\sum\left\{X_{i}: i \in I\right\}$ ) o conjunto $\bigcup_{i \in I}\left(X_{i} \times\{i\}\right)$. O conjunto $\sum_{i \in I} X_{i}$ será chamado de a soma (conjuntista) de $\left\{X_{i}: i \in I\right\}$. Pode-se considerar a função $p_{I, X_{i}}: \sum_{i \in I} X_{i} \rightarrow I$ que é definida pondo-se, para todo $i \in I$ e todo $x \in X_{i}$,

$$
p_{I, X_{i}}(x, i):=i
$$

A função $p_{I, X_{i}}$ será chamada de a projeção de $\sum_{i \in I} X_{i}$ em $I$. Mostra-se facilmente que:

(i) Para todo $J \subseteq I,\left(p_{I, X_{i}}\right)^{-1}[J]=\sum_{i \in J} X_{i}$.

Dada uma família $\left\{\tau_{i}: i \in I\right\}$ de conjuntos tais que, para todo $i \in I, \tau_{i}$ é uma topologia sobre $X_{i}$, verifica-se corriqueiramente que:

(ii) $\left\{\sum_{i \in I} V_{i}: \forall i \in I\left(V_{i} \in \tau_{i}\right)\right\}$ é uma topologia sobre $\sum_{i \in I} X_{i}$.

Esta família será chamada de a topologia soma de $\left\{\tau_{i}: i \in I\right\}$ (sobre $\sum_{i \in I} X_{i}$ ) e denotada por $\bigoplus_{i \in I} \tau_{i}$. O espaço topológico $\left\langle\sum_{i \in I} X_{i}, \bigoplus_{i \in I} \tau_{i}\right\rangle$ será chamado de a soma topológica de $\left\{\left\langle X_{i}, \tau_{i}\right\rangle: i \in I\right\}$ e denotado por $\sum_{i \in I}\left\langle X_{i}, \tau_{i}\right\rangle$. Usando o item $(i)$, conclui-se que:

(iii) $p_{I, X_{i}}$ é contínua com respeito a $\bigoplus_{i \in I} \tau_{i}$ e $\mathcal{P}(I)$. 
Comentário 1.1.23 ([Eng1989, cap. 2, seç. 3], [Wil1970, cap. 3, seç. 8]). Dados um conjunto $I$ e uma família $\left\{X_{i}: i \in I\right\}$ de conjuntos, denotaremos por $\prod_{i \in I} X_{i}$ (ou por $\left.\prod\left\{X_{i}: i \in I\right\}\right)$ o conjunto

$$
\left\{\xi: \xi \text { é uma função de } I \text { em } \bigcup_{i \in I} X_{i} \wedge \forall i \in I\left(\xi(i) \in X_{i}\right)\right\} .
$$

O conjunto $\prod_{i \in I} X_{i}$ será chamado de o produto (cartesiano) de $\left\{X_{i}: i \in I\right\}$. Dada uma família $\left\{\tau_{i}: i \in I\right\}$ de conjuntos tais que, para todo $i \in I, \tau_{i}$ é uma topologia sobre $X_{i}$, considere a seguinte família:

$$
\mathcal{B}\left(I, \tau_{i}\right):=\left\{\prod_{i \in I} V_{i}: \forall i \in I\left(V_{i} \in \tau_{i}\right) \wedge\left\{l \in I: V_{i} \neq X_{i}\right\} \text { é finito }\right\} .
$$

Usando o Comentário 1.1.10 (ii), verifica-se corriqueiramente que:

(i) $\mathcal{B}\left(I, \tau_{i}\right)$ gera uma topologia sobre $\prod_{i \in I} X_{i}$

A topologia gerada $\left(\mathcal{B}\left(I, \tau_{i}\right)\right)^{\cup}$ será chamada de a topologia produto de $\left\{\tau_{i}: i \in I\right\}$ (sobre $\left.\prod_{i \in I} X_{i}\right)$ e denotada por $\bigotimes_{i \in I} \tau_{i}$. O espaço topológico $\left\langle\prod_{i \in I} X_{i}, \bigotimes_{i \in I} \tau_{i}\right\rangle$ será chamado de o produto topológico de $\left\{\left\langle X_{i}, \tau_{i}\right\rangle: i \in I\right\}$ e denotado por $\prod_{i \in I}\left\langle X_{i}, \tau_{i}\right\rangle$. Dados um $\alpha \in \prod_{i \in I} X_{i}$ e um $V \in \mathcal{N}_{\bigotimes_{i \in I} \tau_{i}}(\alpha) \cap \mathcal{B}\left(I, \tau_{i}\right)$, será dito que $V$ é uma vizinhança canônica de $\alpha$ em $\prod_{i \in I}\left\langle X_{i}, \tau_{i}\right\rangle$. Agora, suponha que $I$ seja não vazio. Dados um $j \in I$ e um $A \subseteq X_{j}$, para cada $i \in I$, considere o seguinte conjunto:

$$
A_{i}:= \begin{cases}A, & \text { se } i=j \\ X_{i}, & \text { caso contrário. }\end{cases}
$$

Também considere a função $p_{I, X_{i}, j}: \prod_{i \in I} X_{i} \rightarrow X_{j}$ que é definida pondo-se, para todo $\xi \in \prod_{i \in I} X_{i}$

$$
p_{I, X_{i}, j}(\xi):=\xi(j)
$$

A função $p_{I, X_{i}, j}$ será chamada de a projeção de $\prod_{i \in I} X_{i}$ na j-ésima coordenada. Mostra-se facilmente que:

(ii) (1) $\left(p_{I, X_{i}, j}\right)^{-1}[A]=\prod_{i \in I} A_{i}$. 
(2) $p_{I, X_{i}, j}$ é contínua com respeito a $\bigotimes_{i \in I} \tau_{i}$ e $\tau_{j}$.

Dada uma família $\left\{X_{0}, X_{1}\right\}$ de conjuntos, considere a função

$$
\gamma_{X_{0}, X_{1}}: X_{0} \times X_{1} \rightarrow \prod\left\{X_{0}, X_{1}\right\}
$$

que é definida da seguinte maneira: para todo $x_{0} \in X_{0}$ e todo $x_{1} \in X_{1}, \gamma_{X_{0}, X_{1}}\left(x_{0}, x_{1}\right)$ é a função de 2 em $X_{0} \cup X_{1}$ que é definida pondo-se, para todo $k \in 2$,

$$
\left(\gamma_{X_{0}, X_{1}}\left(x_{0}, x_{1}\right)\right)(k):=x_{k}
$$

Dadas uma topologia $\tau_{0}$ sobre $X_{0}$ e uma topologia $\tau_{1}$ sobre $X_{1}$, é fácil verificar que:

(iii) $\gamma_{X_{0}, X_{1}}$ é um homeomorfismo com respeito a $\tau_{0} \otimes \tau_{1}$ e $\bigotimes\left\{\tau_{0}, \tau_{1}\right\}$.

Teorema 1.1.24. Sejam I um conjunto e $\left\{\left\langle X_{i}, \tau_{i}\right\rangle: i \in I\right\}$ uma família de espaços topológicos. Então:

(i) Se, para todo $i \in I,\left\langle X_{i}, \tau_{i}\right\rangle$ for $T_{1}$, então
(1) $\sum_{i \in I}\left\langle X_{i}, \tau_{i}\right\rangle$ é $T_{1}$.
(2) $\prod_{i \in I}\left\langle X_{i}, \tau_{i}\right\rangle$ é $T_{1}$.

(ii) Se, para todo $i \in I,\left\langle X_{i}, \tau_{i}\right\rangle$ for de Tychonoff, então
(1) $\sum_{i \in I}\left\langle X_{i}, \tau_{i}\right\rangle$ é de Tychonoff.
(2) $\prod_{i \in I}\left\langle X_{i}, \tau_{i}\right\rangle$ é de Tychonoff.

\subsection{Noções métricas e analítico-funcionais}

Nesta seção, são estabelecidas generalizações de definições e resultados comumente estabelecidos no contexto ingênuo ${ }^{4}$ dos espaços métricos ou dos espaços normados e de Banach reais ou complexos.

\footnotetext{
${ }^{4}$ Este termo é uma tradução da palavra francesa naïve, que é utilizada por teóricos conjuntistas com a conotação de algo despreocupado ou pouco cauteloso em relação ao rigor lógico da Teoria Axiomática dos Conjuntos.
} 
Definição 1.2.1. Sejam $X$ um conjunto e $d: X \times X \rightarrow \mathbb{R}$ uma função. Considere as seguintes condições:

(a) (anulamento) Para todo $x \in X, d(x, x)=0$.

(b) (não negatividade) Para quaisquer $x, y \in X, 0 \leqslant d(x, y)$.

(c) (condição $\left.T_{0}\right)$ Para quaisquer $x, y \in X$, se $d(x, y)=0$ e $d(y, x)=0$, então $x=y$.

(d) (não degeneração) Para quaisquer $x, y \in X$, se $d(x, y)=0$, então $x=y$.

(e) (simetria) Para quaisquer $x, y \in X, d(x, y)=d(y, x)$.

(f) (desigualdade triangular) Para quaisquer $x, y, z \in X, d(x, y) \leqslant d(x, z)+d(z, y)$.

(g) (desigualdade triangular forte) Para quaisquer $x, y, z \in X$,

$$
d(x, y) \leqslant \max \{d(x, z), d(z, y)\} \text {. }
$$

Diremos que:

(i) $d$ é uma pré-métrica sobre $X$ se valerem as condições $(a)$ e $(b)$. Se $d$ for uma pré-métrica sobre $X$, diremos que o par $\langle X, d\rangle$ é um espaço pré-métrico.

(ii) $d$ é uma hemimétrica sobre $X$ se $d$ for uma pré-métrica sobre $X$ e valer a condição $(f)$. Se $d$ for uma hemimétrica sobre $X$, diremos que o par $\langle X, d\rangle$ é um espaço hemimétrico. ${ }^{5}$

(iii) $d$ é uma ultra-hemimétrica sobre $X$ se $d$ for uma pré-métrica sobre $X$ e valer a condição $(g)$. Se $d$ for uma ultra-hemimétrica sobre $X$, diremos que o par $\langle X, d\rangle$ é um espaço ultra-hemimétrico.

(iv) $d$ é uma dimétrica sobre $X$ se $d$ for uma hemimétrica sobre $X$ e valer a condição (c). Se $d$ for uma dimétrica sobre $X$, diremos que o par $\langle X, d\rangle$ é um espaço dimétrico. ${ }^{6}$

(v) $d$ é uma quase métrica sobre $X$ se $d$ for uma hemimétrica sobre $X$ e valer a condição $(d)$. Se $d$ for uma quase métrica sobre $X$, diremos que o par $\langle X, d\rangle$ é um espaço quase métrico.

${ }^{5} \mathrm{Na}$ literatura a que tivemos acesso, é mais comum a utilização das expressões quase pseudométrica e quase pseudométrico em lugar dos termos hemimétrica e hemimétrico, respectivamente. Preferimos adotar estes termos por terem escritas mais curtas.

${ }^{6}$ Os termos dimétrica e dimétrico podem ser encontrados em [Gab2016] e têm o mesmo significado das expressões $T_{0}$-quase métrica e $T_{0}$-quase métrico, respectivamente. 
(vi) $d$ é uma pseudométrica sobre $X$ se $d$ for uma hemimétrica sobre $X$ e valer a condição (e). Se $d$ for uma pseudométrica sobre $X$, diremos que o par $\langle X, d\rangle$ é um espaço pseudométrico.

(vii) $d$ é uma ultrapseudométrica sobre $X$ se $d$ for uma ultra-hemimétrica sobre $X$ e valer a condição $(e)$. Se $d$ for uma ultrapseudométrica sobre $X$, diremos que o par $\langle X, d\rangle$ é um espaço ultrapseudométrico.

(viii) $d$ é uma métrica sobre $X$ se $d$ for uma pseudométrica sobre $X$ e valer a condição $(d)$. Se $d$ for uma métrica sobre $X$, diremos que o par $\langle X, d\rangle$ é um espaço métrico.

(ix) $d$ é uma ultramétrica sobre $X$ se $d$ for uma ultrapseudométrica sobre $X$ e valer a condição $(d)$. Se $d$ for uma ultramétrica sobre $X$, diremos que o par $\langle X, d\rangle$ é um espaço ultramétrico.

Suponha que $d$ seja uma pré-métrica sobre $X$. Para qualquer $x \in X$ e qualquer $\varepsilon \in \mathbb{R}^{+}$,

(1) o conjunto $\{z \in X: d(z, x)<\varepsilon\}$ será chamado de a bola aberta segundo $d$ de centro $x$ e raio $\varepsilon$ e denotado por $B_{d}(x, \varepsilon)$.

(2) o conjunto $\{z \in X: d(z, x) \leqslant \varepsilon\}$ será chamado de a bola fechada segundo $d$ de centro $x$ e raio $\varepsilon$ e denotado por $B_{d}[x, \varepsilon]$.

Suponha que $X$ seja não vazio. Para quaisquer $x, y \in X$,

(3) o número real $d(x, y)$ será chamado de a distância de $x$ a $y$ segundo $d$. Se valer a condição $(e)$, o número real $d(x, y)$ será chamado de a distância entre $x$ e $y$ segundo $d$.

Para qualquer subconjunto não vazio $A$ de $X$ e qualquer $x \in X$,

(4) o número real $\inf \{d(x, z): z \in A\}$ será chamado de a distância de $x$ a $A$ segundo $d$ e denotado por $d(x, A)$.

Comentário 1.2.2. Dado um espaço pré-métrico $\langle X, d\rangle$, é imediato concluir que:

(i) Sobre $X$,

(1) d é uma métrica se, e somente se, $d$ for uma pseudométrica e uma quase métrica.

(2) $d$ é uma ultramétrica se, e somente se, $d$ for uma ultrapseudométrica e uma quase métrica. 
É fácil ver que:

(ii) Sobre $X$,

(1) (1) se $d$ for uma quase métrica, então $d$ é uma dimétrica.

(2) se $d$ satisfizer a condição de simetria, então $d$ é uma quase métrica se, e somente se, $d$ for uma dimétrica.

(2) (1) se $d$ for uma ultra-hemimétrica, então $d$ é uma hemimétrica.

(2) se $d$ for uma ultrapseudométrica, então $d$ é uma pseudométrica.

(3) se $d$ for uma ultramétrica, então $d$ é uma métrica.

Mostra-se que:

(iii) (1) $d$ é uma pseudométrica sobre $X$ se, e somente se, $d$ satisfizer a condição de anulamento, de simetria e de desigualdade triangular.

(2) $d$ é uma métrica sobre $X$ se, e somente se, $d$ satisfizer a condição $T_{0}$, de anulamento, de simetria e de desigualdade triangular.

Mostra-se também que:

(iv) São equivalentes:

(1) $d$ é uma pseudométrica sobre $X$.

(2) Para todo subconjunto não vazio $A$ de $X$ e quaisquer $x, y \in X$,

$$
|d(x, A)-d(y, A)| \leqslant d(x, y)
$$

(3) Para quaisquer $x, y, z \in X,|d(x, z)-d(y, z)| \leqslant d(x, y)$.

Denote por $d_{0, X}$ a função identicamente nula de $X \times X$ em $\mathbb{R}$. É fácil ver que:

(v) (1) $d_{0, X}$ é uma ultrapseudométrica sobre $X$.

(2) Se $X$ não for vazio nem for unitário, então $d_{0, X}$ não é uma dimétrica sobre $X$.

(3) Para todo $x \in X$ e todo $\varepsilon \in \mathbb{R}^{+}, B_{d_{0, X}}(x, \varepsilon)=X$.

A ultrapseudométrica $d_{0, X}$ será chamada de a pseudométrica nula de $X$. Considere a função $d_{1, X}: X \times X \rightarrow \mathbb{R}$ que é definida pondo-se, para quaisquer $x, y \in X$,

$$
d_{1, X}(x, y):= \begin{cases}0, & \text { se } x=y \\ 1, & \text { caso contrário. }\end{cases}
$$

É corriqueiro verificar que: 
(vi) (1) $d_{1, X}$ é uma ultramétrica sobre $X$.

(2) Para todo $x \in X$ e todo $\varepsilon \in \mathbb{R}^{+}, B_{d_{1, X}}(x, \varepsilon)=\left\{\begin{aligned}\{x\}, & \text { se } \varepsilon \in] 0,1] \\ X, & \text { caso contrário. }\end{aligned}\right.$

A ultramétrica $d_{1, X}$ será chamada de a métrica zero-um de $X$. Considere as funções $d_{2}, d^{+}, d^{\rightarrow}, d^{\wedge}: \mathbb{R} \times \mathbb{R} \rightarrow \mathbb{R}$ que são definidas pondo-se, para quaisquer $x, y \in \mathbb{R}$,

$$
\begin{aligned}
& d_{2}(x, y):=|x-y|, \\
& d^{+}(x, y):=\max \{0, x-y\}, \\
& d^{\rightarrow}(x, y):=\left\{\begin{aligned}
0, & \text { se } x=y ; \\
|x|+1, & \text { caso contrário; }
\end{aligned}\right. \\
& \text { e } \\
& d^{\wedge}(x, y):=\left\{\begin{aligned}
0, & \text { se } x=y ; \\
\min \{|x|,|y|\}+1, & \text { caso contrário. }
\end{aligned}\right.
\end{aligned}
$$

Também é corriqueiro verificar que:

(vii) Sobre $\mathbb{R}$,

(1) $d^{\wedge}$ é uma pré-métrica satisfazendo a condição de não degeneração e de simetria que não é uma hemimétrica.

(2) $d^{\rightarrow}$ é uma ultra-hemimétrica que é uma quase métrica e que não é uma pseudométrica.

(3) $d^{+}$é uma dimétrica que não é uma ultra-hemimétrica nem é uma quase métrica.

(4) $d_{2}$ é uma métrica que não é uma ultrapseudométrica.

Considere a função $d_{\mathrm{sec}}: 2 \times 2 \rightarrow \mathbb{R}$ que é definida pondo-se, para quaisquer $k, l<2$,

$$
d_{\text {sec }}(k, l):= \begin{cases}0, & \text { se } k=l \\ l, & \text { caso contrário. }\end{cases}
$$

Considere também a função $d_{\min }: 3 \times 3 \rightarrow \mathbb{R}$ que é definida pondo-se, para quaisquer $k, l<3$,

$$
d_{\min }(k, l):=\left\{\begin{aligned}
0, & \text { se } k=l \\
\min \{k, l\}, & \text { caso contrário. }
\end{aligned}\right.
$$

É corriqueiro verificar que:

(ix) Sobre $2, d_{\text {sec }}$ é uma ultra-hemimétrica que é uma dimétrica e que não é uma quase métrica nem é uma pseudométrica. 
(x) Sobre $3, d_{\text {min }}$ é uma pré-métrica satisfazendo a condição de simetria que não é uma hemimétrica.

Proposição 1.2.3. Dados um espaço hemimétrico $\langle X, d\rangle$ tal que $X$ é não vazio, uma sequência $\left\langle x_{n}\right\rangle_{n<\omega}$ em $\left.X, u m \varepsilon \in\right] 0,1[$ e um $l<\omega$, considere as seguintes asserções:

(a) Para todo $k<l, \max \left\{d\left(x_{k}, x_{k+1}\right), d\left(x_{k+1}, x_{k}\right)\right\}<\varepsilon^{k+1}$.

(b) Para todo $k<\omega$, se $k \leqslant l$, então $\max \left\{d\left(x_{k}, x_{l}\right), d\left(x_{l}, x_{k}\right)\right\}<(1-\varepsilon)^{-1} \varepsilon^{k+1}$.

Então, (a) implica (b).

Usando a Proposição 1.2.3, conclui-se facilmente o seguinte

Corolário 1.2.4. Dados um espaço hemimétrico $\langle X, d\rangle$ tal que $X$ é não vazio, uma sequência $\left\langle x_{n}\right\rangle_{n<\omega}$ em X e um $\left.\varepsilon \in\right] 0,1[$, considere as seguintes asserções:

(a) Para todo $n<\omega, \max \left\{d\left(x_{n}, x_{n+1}\right), d\left(x_{n+1}, x_{n}\right)\right\}<\varepsilon^{n+1}$.

(b) Para quaisquer $n, m<\omega$, se $n \leqslant m$, então $\max \left\{d\left(x_{n}, x_{m}\right), d\left(x_{m}, x_{n}\right)\right\}<(1-\varepsilon)^{-1} \varepsilon^{n+1}$.

Então, (a) implica (b).

Comentário 1.2.5. Dado um espaço pré-métrico $\langle X, d\rangle$, mostra-se facilmente que:

(i) A família $\left\{V \in \mathcal{P}(X): \forall x \in V \exists \delta \in \mathbb{R}^{+}\left(B_{d}(x, \delta) \subseteq V\right)\right\}$ é uma topologia sobre $X$.

Esta família será chamada de a topologia induzida por $d$ (sobre $X$ ) e denotada por $\tau_{d}$. Lembre-se que $\tau_{2}$ denota a topologia da ordem sobre o conjunto $\mathbb{R}$ segundo a ordem total do corpo ordenado $\mathbb{R}$ (cf. Comentário 1.1.15). É fácil ver que:

(ii) $\tau_{d_{2}}=\tau_{2}$

Em virtude do item (ii), a métrica $d_{2}$ será chamada de a métrica usual sobre $\mathbb{R}$. Usando os itens (v) (3) e (vi) (2) do Comentário 1.2.2, conclui-se que:

(iii) (1) $\tau_{d_{0, X}}=\{\emptyset, X\}$.

(2) $\tau_{d_{1, X}}=\mathcal{P}(X)$

Em virtude do item (iii) (2), se $d=d_{1, X}$, será dito que $\langle X, d\rangle$ é um espaço métrico discreto. Dada uma pré-métrica $d^{\prime}$ sobre $X$, se $\tau_{d}=\tau_{d^{\prime}}$, será dito que $d$ é equivalente a $d^{\prime}$. É fácil verificar que: 
(iv) Se existir um $\delta \in \mathbb{R}^{+}$tal que $\operatorname{im}(d) \subseteq\{0\} \cup[\delta,+\infty[$ e $d$ satisfizer a condição de não degeneração, então

(1) para todo $x \in X, x$ é um ponto isolado de $\left\langle X, \tau_{d}\right\rangle$.

(2) $d$ é equivalente a $d_{1, X}$.

Considere a família $\mathcal{B}_{d}:=\left\{B_{d}(z, \varepsilon): z \in X \wedge \varepsilon \in \mathbb{R}^{+}\right\}$. Vê-se facilmente que:

(v) $\mathcal{B}_{d}$ é uma network para $\tau_{d}$.

Mostra-se que:

(vi) (1) Se $d$ for uma hemimétrica sobre $X$ ou equivalente a $d_{1, X}$, então $\mathcal{B}_{d}$ é uma base para $\tau_{d}$.

(2) Se $d$ for uma pseudométrica sobre $X$ ou equivalente a $d_{1, X}$, então, para todo $x \in X$ e todo $\varepsilon \in \mathbb{R}^{+}, B_{d}[x, \varepsilon]$ é um fechado em $\left\langle X, \tau_{d}\right\rangle$.

É corriqueiro verificar que:

(vii) Para todo $\varepsilon \in \mathbb{R}^{+}$,

(1) (1) $B_{d_{\mathrm{sec}}}(0, \varepsilon)=2$.

(2) $B_{d_{\mathrm{sec}}}(1, \varepsilon)=\left\{\begin{aligned}\{1\}, & \text { se } \varepsilon \in] 0,1] \\ 2, & \text { caso contrário. }\end{aligned}\right.$

(2) (1) $B_{d_{\min }}(0, \varepsilon)=3$.

(2) $B_{d_{\min }}(1, \varepsilon)= \begin{cases}2, & \text { se } \varepsilon \in] 0,1] \\ 3, & \text { caso contrário. }\end{cases}$

(3) $B_{d_{\text {min }}}(2, \varepsilon)=\left\{\begin{aligned}\{0,2\}, & \text { se } \varepsilon \in] 0,1] ; \\ 3, & \text { caso contrário. }\end{aligned}\right.$

Usando o item (vii) (1), é corriqueiro mostrar que:

(viii) (1) $\tau_{d_{\mathrm{sec}}}=\{\emptyset,\{1\}, 2\}$.

(2) Para todo $\varepsilon \in] 0,1], B_{d_{\mathrm{sec}}}[1, \varepsilon]$ não é um fechado em $\left\langle 2, \tau_{d_{\mathrm{sec}}}\right\rangle$.

A família $\{\emptyset,\{1\}, 2\}$ é chamada de a topologia de Sierpiński em [DN2004, p. 20]. Usando o item (vii)(2), também é corriqueiro mostrar que:

(ix) (1) $d_{\min }$ é equivalente a $d_{0,3}$.

(2) Para todo $k \in 3 \backslash 1$ e todo $\varepsilon \in \mathbb{R}^{+}$, 
(1) se $\varepsilon \in] 0,1]$, então $B_{d_{\min }}(k, \varepsilon)$ não é um aberto em $\left\langle 3, \tau_{d_{\min }}\right\rangle$.

(2) se $\varepsilon \in] 0,1\left[\right.$, então $B_{d_{\min }}[k, \varepsilon]$ não é um fechado em $\left\langle 3, \tau_{d_{\min }}\right\rangle$.

Comentário 1.2.6. Dados um espaço pré-métrico $\langle X, d\rangle$ e um $A \subseteq X$, considere a seguinte família:

$$
\mathcal{B}_{d, A}:=\left\{B_{d}\left(z, \frac{1}{n}\right): z \in A \wedge n \in \omega \backslash 1\right\}
$$

É fácil ver que:

(i) $\mathcal{B}_{d, X}$ é uma network para $\tau_{d}$.

Para cada $x \in X$, denote por $\mathcal{B}_{d, x}$ a família $\mathcal{B}_{d,\{x\}}$. Também é fácil ver que:

(ii) Para todo $x \in X$,

(1) $\mathcal{B}_{d, x}=\left\{B_{d}\left(x, \frac{1}{n}\right): n \in \omega \backslash 1\right\}$.

(2) $\mathcal{B}_{d, x}$ é uma network local para $\tau_{d}$ em $x$.

Em virtude dos itens (i) e (ii) (2) e do Comentário 1.2.5 (vi) (1), tem-se que:

(iii) Se $d$ for uma hemimétrica sobre $X$ ou equivalente a $d_{1, X}$, então

(1) $\mathcal{B}_{d, X}$ é uma base para $\tau_{d}$.

(2) (1) para todo $x \in X, \mathcal{B}_{d, x}$ é uma base local para $\tau_{d}$ em $x$.

(2) $\left\langle X, \tau_{d}\right\rangle$ tem caráter enumerável.

Mostra-se que:

(iv) Se $d$ for uma pseudométrica sobre $X$, então

(1) $A$ ser um denso em $\left\langle X, \tau_{d}\right\rangle$ é condição suficiente para $\mathcal{B}_{d, A}$ ser uma base para $\tau_{d}$

(2) $\left\langle X, \tau_{d}\right\rangle$ ser separável é condição suficiente para $\left\langle X, \tau_{d}\right\rangle$ ter peso enumerável.

Agora, denote por $d_{A}$ a restrição $d \uparrow A \times A$ e note que:

(v) $d_{A}$ é uma pré-métrica sobre $A$.

A pré-métrica $d_{A}$ será chamada de a pré-métrica induzida por $d$ sobre $A$. É claro que:

(vi) (1) Se $d$ for uma hemimétrica sobre $X$, então $d_{A}$ é uma hemimétrica sobre $A$. 
(2) Se $d$ for uma ultra-hemimétrica sobre $X$, então $d_{A}$ é uma ultra-hemimétrica sobre $A$.

(3) Se $d$ satisfizer a condição $T_{0}$, então $d_{A}$ satisfaz a condição $T_{0}$.

(4) Se $d$ satisfizer a condição de não degeneração, então $d_{A}$ satisfaz a condição de não degeneração.

(5) Se $d$ satisfizer a condição de simetria, então $d_{A}$ satisfaz a condição de simetria.

É imediato concluir que:

(vii) Para todo $x \in A$ e todo $\varepsilon \in \mathbb{R}^{+}$,

(1) $B_{d_{A}}(x, \varepsilon)=B_{d}(x, \varepsilon) \cap A$.

(2) $B_{d_{A}}[x, \varepsilon]=B_{d}[x, \varepsilon] \cap A$.

Mostra-se que:

(viii) (1) $\left(\tau_{d}\right)_{A} \subseteq \tau_{d_{A}}$.

(2) Se $A \in\{\emptyset, X\}$ ou $d$ for uma hemimétrica sobre $X$, então $\left(\tau_{d}\right)_{A}=\tau_{d_{A}}$.

(ix) Sobre 3, existe uma pré-métrica $d$ satisfazendo a condição de simetria que não é uma hemimétrica e tal que $\left(\tau_{d}\right)_{\{0,2\}} \neq \tau_{d\{0,2\}}$.

Definição 1.2.7. Sejam $\langle X, d\rangle$ e $\left\langle X^{\prime}, d^{\prime}\right\rangle$ espaços pré-métricos e $\varphi: X \rightarrow X^{\prime}$ uma função. Diremos que:

(i) $\varphi$ é uniformemente contínua segundo $d$ e $d^{\prime}$ se valer a seguinte condição: para todo $\varepsilon \in \mathbb{R}^{+}$, existe um $\delta \in \mathbb{R}^{+}$tal que, para quaisquer $x, y \in X$, se $d(x, y)<\delta$, então $d^{\prime}(\varphi(x), \varphi(y))<\varepsilon$.

(ii) $\varphi$ é não expansiva segundo $d$ e $d^{\prime}$ se valer a seguinte condição: para quaisquer $x, y \in X, d^{\prime}(\varphi(x), \varphi(y)) \leqslant d(x, y)$.

(iii) $\varphi$ preserva distância segundo $d$ e $d^{\prime}$ se valer a seguinte condição: para quaisquer $x, y \in X, d^{\prime}(\varphi(x), \varphi(y))=d(x, y)$.

(iv) $\varphi$ é uma imersão isométrica segundo $d$ e $d^{\prime}$ se valerem as seguintes condições:

(1) $\varphi$ é injetora.

(2) $\varphi$ preserva distância segundo $d$ e $d^{\prime}$. 
(v) $\varphi$ é uma isometria segundo $d$ e $d^{\prime}$ se valerem as seguintes condições:

(1) $\varphi$ é bijetora.

(2) $\varphi$ preserva distância segundo $d$ e $d^{\prime}$.

Teorema 1.2.8. Dados espaços pré-métricos $\langle X, d\rangle$ e $\left\langle X^{\prime}, d^{\prime}\right\rangle$ e uma função $\varphi: X \rightarrow X^{\prime}$, considere as seguintes asserções:

(a) $\varphi$ preserva distância segundo $d$ e $d^{\prime}$.

(b) $\varphi$ é não expansiva segundo $d$ e $d^{\prime}$.

(c) $\varphi$ é uniformemente contínua segundo $d$ e $d^{\prime}$.

(d) $\varphi$ é contínua com respeito a $\tau_{d} e \tau_{d^{\prime}}$.

Então:

(i) (a) implica (b).

(ii) (b) implica (c).

Suponha que d seja uma hemimétrica sobre X. Então:

(iii) (c) implica $(d)$.

Suponha que d seja uma pseudométrica sobre $X$ e que $\left\langle X, \tau_{d}\right\rangle$ seja compacto. Então:

(iv) (c) é equivalente a $(d)$.

Comentário 1.2.9. Dados um espaço pré-métrico $\langle X, d\rangle$ tal que $X$ é não vazio e um subconjunto não vazio $A$ de $X$, pode-se considerar a função $d^{A}: X \rightarrow \mathbb{R}$ que é definida pondo-se, para todo $x \in X$,

$$
d^{A}(x):=d(x, A)
$$

Note que:

(i) (1) $A \subseteq\left(d^{A}\right)^{-1}[\{0\}]$.

(2) $d^{X}$ é a função identicamente nula de $X$ em $\mathbb{R}$.

Mostra-se que:

(ii) (1) $d^{\bar{A}^{\tau_{d}}} \leqslant d^{A}$. 
(2) Se $d$ for uma pseudométrica sobre $X$, então $d^{\bar{A}^{\tau_{d}}}=d^{A}$.

Dado um $a \in X$, denotaremos por $d^{a}$ a função $d^{\{a\}}$. Note que:

(iii) Para todo $x \in X, d^{a}(x)=d(x, a)$.

Usando o item (ii), tem-se que:

(iv) (1) $d^{\overline{\{a\}}^{\tau_{d}}} \leqslant d^{a}$.

(2) Se $d$ for uma pseudométrica sobre $X$, então $d^{\overline{\{a\}}^{\tau_{d}}}=d^{a}$.

Mostra-se que:

(v) (1) Se $d$ satisfizer a condição de simetria, então
(1) $\left(d^{A}\right)^{-1}[\{0\}] \subseteq \bar{A}^{\tau_{d}}$.
(2) $\left(d^{a}\right)^{-1}[\{0\}] \subseteq \overline{\{a\}}^{\tau_{d}}$.

(2) Se $d$ for uma pseudométrica sobre $X$, então

(1) $\left(d^{A}\right)^{-1}[\{0\}]=\bar{A}^{\tau_{d}}$.

(2) $\left(d^{a}\right)^{-1}[\{0\}]=\overline{\{a\}}^{\tau_{d}}$.

Mostra-se também que:

(vi) Sobre 2, existe uma ultra-hemimétrica $d$ que é uma dimétrica, que não satisfaz a condição de simetria e tal que

(1) para todo $k<2, d^{\overline{\{1\}}^{\tau_{d}}}(k) \neq d^{\{1\}}(k)$.

(2) $\left(d^{0}\right)^{-1}[\{0\}] \nsubseteq \overline{\{0\}}^{\tau_{d}}$.

(vii) Sobre 3, existe uma pré-métrica $d$ satisfazendo a condição de simetria que não é uma pseudométrica e tal que $\left(d^{1}\right)^{-1}[\{0\}] \neq \overline{\{1\}}^{\tau_{d}}$.

Suponha que $d$ seja uma pseudométrica sobre $X$. Em virtude do Comentário 1.2.2(iv), tem-se que:

(viii) (1) $d^{A}$ é não expansiva segundo $d$ e $d_{2}$.

(2) $d^{a}$ é não expansiva segundo $d$ e $d_{2}$.

A proposição a seguir justifica por que a condição (c) da Definição 1.2.1 é chamada de condição $T_{0}$ e estabelece, em termos topológicos, condições necessárias e suficientes para que uma pseudométrica seja uma métrica. 
Proposição 1.2.10. Seja $\langle X, d\rangle$ um espaço pré-métrico. Então:

(i) Se $\left\langle X, \tau_{d}\right\rangle$ for $T_{0}$, então d satisfaz a condição $T_{0}$.

Suponha que d seja uma hemimétrica sobre X. Então:

(ii) d é uma dimétrica sobre $X$ se, e somente se, $\left\langle X, \tau_{d}\right\rangle$ for $T_{0}$.

Suponha que d seja uma pseudométrica sobre X. Então:

(iii) São equivalentes:

(1) d é uma métrica sobre X.

(2) $\left\langle X, \tau_{d}\right\rangle$ é de Tychonoff.

(3) $\left\langle X, \tau_{d}\right\rangle$ é regular.

(4) $\left\langle X, \tau_{d}\right\rangle$ é de Hausdorff.

(5) $\left\langle X, \tau_{d}\right\rangle$ é $T_{1}$.

(6) $\left\langle X, \tau_{d}\right\rangle$ é $T_{0}$.

Comentário 1.2.11. Dados espaços pré-métricos $\langle X, d\rangle$ e $\left\langle X^{\prime}, d^{\prime}\right\rangle$, considere a função $d \otimes d^{\prime}:\left(X \times X^{\prime}\right) \times\left(X \times X^{\prime}\right) \rightarrow \mathbb{R}$ que é definida pondo-se, para quaisquer $x, y \in X \mathrm{e}$ quaisquer $x^{\prime}, y^{\prime} \in X^{\prime}$,

$$
\left(d \otimes d^{\prime}\right)\left(\left\langle x, x^{\prime}\right\rangle,\left\langle y, y^{\prime}\right\rangle\right):=d(x, y)+d^{\prime}\left(x^{\prime}, y^{\prime}\right)
$$

Note que:

(i) $d \otimes d^{\prime}$ é uma pré-métrica sobre $X \times X^{\prime}$.

A pré-métrica $d \otimes d^{\prime}$ será chamada de a pré-métrica produto de $d$ e $d^{\prime}$ (sobre $X \times X^{\prime}$ ). $\mathrm{O}$ espaço pré-métrico $\left\langle X \times X^{\prime}, d \otimes d^{\prime}\right\rangle$ será chamado de o espaço produto de $\langle X, d\rangle$ e $\left\langle X^{\prime}, d^{\prime}\right\rangle$ e denotado por $\langle X, d\rangle \otimes\left\langle X^{\prime}, d^{\prime}\right\rangle$. Mostra-se facilmente que:

(ii) (1) Se $d$ for uma hemimétrica sobre $X$ e $d^{\prime}$ for uma hemimétrica sobre $X^{\prime}$, então $d \otimes d^{\prime}$ é uma hemimétrica sobre $X \times X^{\prime}$.

(2) Se $d$ e $d^{\prime}$ satisfizerem a condição $T_{0}$, então $d \otimes d^{\prime}$ satisfaz a condição $T_{0}$.

(3) Se $d$ e $d^{\prime}$ satisfizerem a condição de não degeneração, então $d \otimes d^{\prime}$ satisfaz a condição de não degeneração. 
(4) Se $d$ e $d^{\prime}$ satisfizerem a condição de simetria, então $d \otimes d^{\prime}$ satisfaz a condição de simetria.

Mostra-se que:

(iii) Sobre 2, existe uma ultramétrica $d$ tal que $d \otimes d$ não é uma ultra-hemimétrica sobre $2 \times 2$.

Vê-se facilmente que:

(iv) Para todo $x \in X$, todo $x^{\prime} \in X^{\prime}$ e quaisquer $\varepsilon, \varepsilon^{\prime} \in \mathbb{R}^{+}$,

(1) $B_{d \otimes d^{\prime}}\left(\left\langle x, x^{\prime}\right\rangle, \min \left\{\varepsilon, \varepsilon^{\prime}\right\}\right) \subseteq B_{d}(x, \varepsilon) \times B_{d^{\prime}}\left(x^{\prime}, \varepsilon^{\prime}\right)$.

(2) $B_{d}(x, \varepsilon) \times B_{d^{\prime}}\left(x^{\prime}, \varepsilon^{\prime}\right) \subseteq B_{d \otimes d^{\prime}}\left(\left\langle x, x^{\prime}\right\rangle, \varepsilon+\varepsilon^{\prime}\right)$.

Com o uso do item (iv) e do Comentário 1.2.5(vi)(1), é corriqueiro mostrar que:

(v) (1) $\tau_{d} \otimes \tau_{d^{\prime}} \subseteq \tau_{d \otimes d^{\prime}}$

(2) Se $d$ for uma hemimétrica sobre $X$ ou equivalente a $d_{1, X}$ e $d^{\prime}$ for uma hemimétrica sobre $X^{\prime}$ ou equivalente a $d_{1, X^{\prime}}$, então $\tau_{d} \otimes \tau_{d^{\prime}}=\tau_{d \otimes d^{\prime}}$.

Mostra-se que:

(vi) Se $d$ for uma pseudométrica sobre $X$, então $d$ é não expansiva segundo $d \otimes d$ e $d_{2}$.

(vii) Se $d$ for uma pseudométrica sobre $X$ ou equivalente a $d_{1, X}$, então $d$ é contínua com respeito a $\tau_{d} \otimes \tau_{d}$ e $\tau_{2}$.

Mostra-se também que:

(vii) Sobre $\mathbb{R}$, existe uma ultra-hemimétrica $d$ que é uma quase métrica, que não é uma pseudométrica e tal que $d$ não é não expansiva segundo $d \otimes d$ e $d_{2}$.

(ix) Sobre 2, existe uma ultra-hemimétrica $d$ que é uma dimétrica, que não é uma pseudométrica nem é equivalente a $d_{1,2}$ e tal que $d$ não é contínua com respeito a $\tau_{d} \otimes \tau_{d}$ e $\tau_{2}$

No que segue, é apresentada uma construção de espaço pré-métrico quociente que generaliza a bem conhecida construção de um espaço métrico a partir de um espaço pseudométrico. 
Comentário 1.2.12. Dados um espaço pré-métrico $\langle X, d\rangle$ e uma relação binária $R$ sobre $X$, será dito que $d$ é invariante por sucessão segundo $R$ se valer a seguinte condição: para quaisquer $x, y, z, w \in X$, se $x R z$ e $y R w$, então $d(x, y)=d(z, w)$. Suponha que $R$ seja uma relação de equivalência sobre $X$ e que $d$ seja invariante por sucessão segundo $R$. É claro que:

(i) Para quaisquer $x, y, z, w \in X$, se $[x]_{R}=[z]_{R}$ e $[y]_{R}=[w]_{R}$, então

$$
d(x, y)=d(z, w)
$$

Logo, pode-se considerar a função $d / R: X / R \times X / R \rightarrow \mathbb{R}$ que é definida pondo-se, para quaisquer $x, y \in X$,

$$
d / R\left([x]_{R},[y]_{R}\right):=d(x, y)
$$

Note que:

(ii) $d / R$ é uma pré-métrica sobre $X / R$.

A pré-métrica $d / R$ será chamada de a pré-métrica quociente induzida por $d$ sobre $X / R$. O espaço pré-métrico $\langle X / R, d / R\rangle$ será chamado de o espaço quociente de $\langle X, d\rangle$ por $R$ e denotado por $\langle X, d\rangle / R$. Lembrando que $q_{R}$ denota a função quociente para $\langle X, R\rangle$ (cf. Comentário 1.1.8), tem-se que:

(iii) $q_{R}$ preserva distância segundo $d \mathrm{e}^{d / R}$.

Mostra-se facilmente que:

(iv) (1) Se $d$ for uma hemimétrica sobre $X$, então $d / R$ é uma hemimétrica sobre $X / R$.

(2) Se $d$ for uma ultra-hemimétrica sobre $X$, então $d / R$ é uma ultra-hemimétrica sobre $X / R$.

(3) Se $d$ satisfizer a condição $T_{0}$, então

(1) $d / R$ satisfaz a condição $T_{0}$.

(2) $q_{R}$ é uma isometria segundo $d \mathrm{e}^{d / R}$.

(4) Se $d$ satisfizer a condição de não degeneração, então $d / R$ satisfaz a condição de não degeneração.

(5) Se $d$ satisfizer a condição de simetria, então $d / R$ satisfaz a condição de simetria. 
Dados um espaço pré-métrico $\left\langle X^{\prime}, d^{\prime}\right\rangle$ e uma função $\varphi: X \rightarrow X^{\prime}$, considere a relação binária $R_{\varphi}^{d^{\prime}}$ sobre $X$ que é definida pela seguinte sentença:

$$
\text { para quaisquer } x, y \in X, x R_{\varphi}^{d^{\prime}} y \text { se, e só se, } d^{\prime}(\varphi(x), \varphi(y))=0 \text {. }
$$

A relação binária $R_{\varphi}^{d^{\prime}}$ será chamada de a relação induzida por $d^{\prime}$ e $\varphi$ (sobre $X$ ). Verifica-se facilmente que:

(v) (1) $R_{\varphi}^{d^{\prime}}$ é reflexiva sobre $X$.

(2) $d^{\prime}$ ser uma hemimétrica sobre $X^{\prime}$ é condição suficiente para $R_{\varphi}^{d^{\prime}}$ ser uma pré-ordem sobre $X$.

(3) $d^{\prime}$ ser uma pseudométrica sobre $X^{\prime}$ é condição suficiente para $R_{\varphi}^{d^{\prime}}$ ser uma relação de equivalência sobre $X$.

A relação binária $R_{\mathrm{id}_{X}}^{d}$ será chamada de a relação induzida por $d$ (sobre $X$ ) e denotada por $R^{d}$. Sendo assim, $R^{d}$ é dada pela seguinte sentença:

$$
\text { para quaisquer } x, y \in X, x R^{d} y \text { se, e somente se, } d(x, y)=0 \text {. }
$$

Mostra-se que:

(vi) Sobre 3, existe uma pré-métrica $d$ satisfazendo a condição de simetria que não é uma hemimétrica e tal que $R^{d}$ não é uma pré-ordem sobre 3 .

(vii) Sobre 2, existe uma ultra-hemimétrica $d$ que é uma dimétrica, que não é uma pseudométrica e tal que $R^{d}$ não é uma relação de equivalência sobre 2 .

É fácil ver que:

(viii) (1) Se $\varphi$ for não expansiva segundo $d$ e $d^{\prime}$, então $R^{d} \subseteq R_{\varphi}^{d^{\prime}}$.

(2) Se $\varphi$ preservar distância segundo $d$ e $d^{\prime}$, então $R_{\varphi}^{d^{\prime}}=R^{d}$.

Também é fácil ver que:

(ix) Se $X$ não for vazio nem for unitário, então

(1) $R^{d_{0, X}} \nsubseteq R^{d_{1, X}}$.

(2) $R^{d_{1, X}} \neq R^{d_{0, X}}$.

Mostra-se que:

(x) Se $d$ for uma pseudométrica sobre $X$, então 
(1) $d$ é invariante por sucessão segundo $R^{d}$.

(2) $d / R^{d}$ é uma métrica sobre $X / R^{d}$.

Suponha que $d$ seja uma pseudométrica sobre $X$. A métrica $d / R^{d}$ será chamada de a métrica quociente induzida por $d$ (sobre $X / R^{d}$ ). O espaço métrico $\langle X, d\rangle / R^{d}$ é chamado de a reflexão métrica de $\langle X, d\rangle$ em [HK2015, p. 78].

O teorema a seguir é um resultado análogo ao Primeiro Teorema do Isomorfismo para álgebras universais (v. [BuS1981, cap. 2, seç. 6]).

Teorema 1.2.13. Sejam $\langle X, d\rangle$ e $\left\langle X^{\prime}, d^{\prime}\right\rangle$ espaços pseudométricos e $\varphi: X \rightarrow X^{\prime}$ uma função tais que $\varphi$ preserva distância segundo $d$ e $d^{\prime}$ e $\left(d^{\prime}\right)_{\operatorname{im}(\varphi)}$ é uma métrica sobre $\operatorname{im}(\varphi)$. Então:

(i) Existe uma única imersão isométrica $\psi: X / R^{d} \rightarrow X^{\prime}$ segundo $d / R^{d}$ e d' tal que

$$
\psi \circ q_{R^{d}}=\varphi
$$

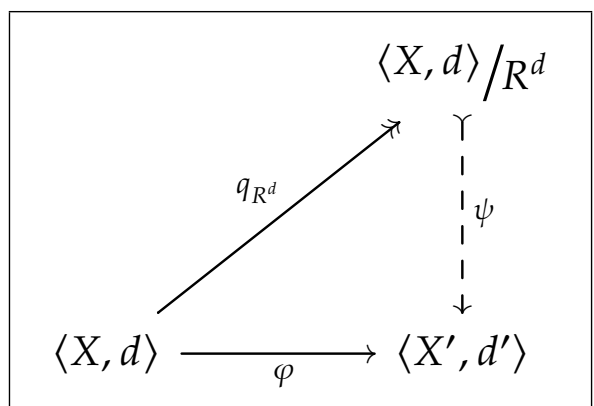

Diagrama i.1

(ii) Se $\varphi$ for sobrejetora, então

(1) d' é uma métrica sobre $X^{\prime}$.

(2) $\langle X, d\rangle / R^{d}$ é isométrico a $\left\langle X^{\prime}, d^{\prime}\right\rangle$.

Definição 1.2.14. Seja $I$ um conjunto. Sejam $\langle X, d\rangle$ um espaço pré-métrico, $\varphi: I \rightarrow X$ uma função e $A \subseteq X$. Diremos que:

(i) $d$ é limitada se valer a seguinte condição: existe um $\delta \in \mathbb{R}^{+}$tal que $\operatorname{im}(d) \subseteq[0, \delta]$. Se $d$ for limitada, diremos que $\langle X, d\rangle$ é limitado.

(ii) A é um limitado segundo $d$ se $d_{A}$ for limitada.

(iii) $\varphi$ é limitada segundo $d$ se o conjunto $\operatorname{im}(\varphi)$ for um limitado segundo $d$. 
Suponha que $X$ seja não vazio. Para qualquer subconjunto não vazio $B$ de $X$, se $B$ for um limitado segundo $d$, o número real $\sup \left(\operatorname{im}\left(d_{B}\right)\right)$ será chamado de o diâmetro de $B$ segundo $d$ e denotado por $\operatorname{diam}_{d}(B)$ (ou, simplesmente, por diam(B)).

Proposição 1.2.15. Dados um espaço pré-métrico $\langle X, d\rangle$ e um $A \subseteq X$, considere as seguintes asserções:

(a) A é um limitado segundo d.

(b) Para todo $B \subseteq A$, B é um limitado segundo d.

(c) Para todo $B \subseteq X$, se $B$ for um limitado segundo d, então o conjunto $A \cup B$ é um limitado segundo $d$.

(d) $A=\emptyset$ ou existem um $x \in X$ e um $\delta \in \mathbb{R}^{+}$tais que $A \subseteq B_{d}[x, \delta]$.

(e) $\left\langle A,\left(\tau_{d}\right)_{A}\right\rangle$ é compacto.

Então:

(i) (a) é equivalente $a(b)$.

(ii) (a) implica (d).

(iii) (c) implica (a).

Suponha que d seja uma hemimétrica sobre X. Então:

(iv) (a) é equivalente a (c).

Suponha que d seja uma pseudométrica sobre X. Então:

(v) (a) é equivalente a (d).

(vi) (e) implica (a).

Proposição 1.2.16. Dados um conjunto $I$, um espaço pré-métrico $\langle X, d\rangle$ e uma função $\varphi: I \rightarrow X$, considere as seguintes asserções:

(a) $\varphi$ é limitada segundo d.

(b) Para todo conjunto $Z$ e toda função $\zeta: Z \rightarrow I, \varphi \circ \zeta$ é limitada segundo d. 
(c) Para toda função $\zeta: I \rightarrow X$, se $\zeta$ for limitada segundo d, então existe $u m \delta \in \mathbb{R}^{+}$tal que $\{d(\varphi(i), \zeta(i)): i \in I\} \subseteq[0, \delta]$

Então:

(i) (a) é equivalente $a(b)$.

Suponha que d seja uma hemimétrica sobre X. Então:

(ii) (a) implica (c).

Suponha que d seja uma pseudométrica sobre X. Então:

(iii) (a) é equivalente a (c).

O teorema a seguir é o Teorema de Heine-Borel da Análise Real (v. [V1995]).

Teorema 1.2.17. Sejam $\lambda, v \in \mathbb{R}$ tais que $\lambda \leqslant v$. Então:

(i) $\left\langle[\lambda, v],\left(\tau_{2}\right)_{[\lambda, v]}\right\rangle$ é compacto.

(ii) Para todo $A \subseteq X$, se $A$ for um fechado em $\left\langle\mathbb{R}, \tau_{2}\right\rangle$ que é um limitado segundo $d_{2}$, então $\left\langle A,\left(\tau_{2}\right)_{A}\right\rangle$ é compacto.

Definição 1.2.18. Sejam $\langle X, d\rangle$ um espaço pré-métrico tal que $X$ é não vazio e $\left\langle x_{n}\right\rangle_{n<\omega}$ uma sequência em $X$. Diremos que $\left\langle x_{n}\right\rangle_{n<\omega}$ é de Cauchy segundo $d$ se valer a seguinte condição: para todo $\varepsilon \in \mathbb{R}^{+}$, existe um $k<\omega$ tal que, para quaisquer $n, m \in \omega \backslash k$, $d\left(x_{n}, x_{m}\right)<\varepsilon$.

Proposição 1.2.19. Dados um espaço pré-métrico $\langle X, d\rangle$ tal que $X$ é não vazio, uma sequência s em X e um $a \in X$, considere as seguintes asserções:

(a) A sequência $d^{a}$ o s converge para 0 com respeito a $\tau_{2}$.

(b) Para todo $\varepsilon \in \mathbb{R}^{+}$, s está eventualmente em $B_{d}(a, \varepsilon)$.

(c) A sequência s converge para a com respeito $a \tau_{d}$.

(d) Existe uma subsequência $t$ de s tal que t converge para a com respeito $a \tau_{d}$.

Então:

(i) (a) é equivalente a (b). 
(ii) (a) implica (c).

(iii) (c) implica (d).

Suponha que d seja uma hemimétrica sobre X. Então:

(iv) (a) é equivalente a (c).

Suponha que d seja uma hemimétrica sobre X e que s seja de Cauchy segundo d. Então:

(v) (c) é equivalente a $(d)$.

Proposição 1.2.20. Dados um espaço pré-métrico $\langle X, d\rangle$ tal que X é não vazio, uma sequência s em $X$ e um $A \subseteq X$ tais que $\operatorname{im}(s) \subseteq A$, considere as seguintes asserções:

(a) A sequência s é de Cauchy segundo d.

(b) A sequência s é de Cauchy segundo $d_{A}$.

(c) A sequência s é limitada segundo d.

(d) A sequência s é limitada segundo $d_{A}$.

(e) A sequência s é convergente com respeito a $\tau_{d}$.

Então:

(i) (a) é equivalente a (b).

(ii) (c) é equivalente a $(d)$.

Suponha que d seja uma hemimétrica sobre X. Então:

(iii) (a) implica (c).

(iv) (e) implica (a).

Teorema 1.2.21. Dados um espaço pré-métrico $\langle X, d\rangle$ tal que $X$ é não vazio e uma sequência $\left\langle x_{n}\right\rangle_{n<\omega}$ em $X$, considere as seguintes asserções:

(a) A sequência $\left\langle x_{n}\right\rangle_{n<\omega}$ é de Cauchy segundo d.

(b) Para todo $n<\omega, \max \left\{d\left(x_{n}, x_{n+1}\right), d\left(x_{n+1}, x_{n}\right)\right\}<2^{-(n+1)}$. 
(c) Existe uma subsequência $\left\langle x_{n_{k}}\right\rangle_{k<\omega}$ de $\left\langle x_{n}\right\rangle_{n<\omega}$ tal que, para todo $k<\omega$,

$$
\max \left\{d\left(x_{n_{k}}, x_{n_{k+1}}\right), d\left(x_{n_{k+1}}, x_{n_{k}}\right)\right\}<2^{-(k+1)} .
$$

Então:

(i) (a) implica (c).

(ii) (b) implica (c).

Suponha que d seja uma hemimétrica sobre X. Então:

(iii) (b) implica (a).

Definição 1.2.22. Seja $\langle X, d\rangle$ um espaço pré-métrico. Diremos que:

(i) $d$ é de Heine-Borel se valer a seguinte condição: para todo $x \in X$ e todo $\varepsilon \in \mathbb{R}^{+}$, $\left\langle B_{d}[x, \varepsilon],\left(\tau_{d}\right)_{B_{d}[x, \varepsilon]}\right\rangle$ é compacto. Se $d$ for de Heine-Borel, diremos que $\langle X, d\rangle$ é de Heine-Borel. ${ }^{7}$

(ii) $d$ é completa se valer a seguinte condição: para toda sequência $s$ em $X$, se $s$ for de Cauchy segundo $d$, então $s$ é convergente com respeito a $\tau_{d}$. Se $d$ for completa, diremos que $\langle X, d\rangle$ é completo.

Proposição 1.2.23. Dado um espaço pré-métrico $\langle X, d\rangle$, considere as seguintes asserções:

(a) dé de Heine-Borel.

(b) d é completa.

(c) $\left\langle X, \tau_{d}\right\rangle$ é compacto.

(d) Para todo $A \subseteq X$, se $A$ for um fechado em $\left\langle X, \tau_{d}\right\rangle$ que é um limitado segundo $d$, então $\left\langle A,\left(\tau_{d}\right)_{A}\right\rangle$ é compacto.

Então:

(i) (a) implica $(d)$.

${ }^{7}$ Esta expressão é utilizada, no contexto dos espaços métricos, em [WJ1987]. No entanto, é comum a utilização das expressões métrica própria e espaço métrico próprio em lugar das expressões métrica de Heine-Borel e espaço métrico de Heine-Borel, respectivamente. 
Suponha que d seja uma hemimétrica sobre X. Então:

(ii) (a) implica (b).

Suponha que d seja uma pseudométrica sobre X. Então:

(iii) (a) é equivalente a (d).

(iv) (c) implica (a).

Com o uso dos itens (iii) e (i) do Teorema 1.2.21 e da Proposição 1.2.19(v), é fácil concluir o seguinte

Corolário 1.2.24. Seja $\langle X, d\rangle$ um espaço hemimétrico. Então, são equivalentes:

(i) dé completa.

(ii) Para toda sequência $\left\langle x_{n}\right\rangle_{n<\omega}$ em $X$, se, para todo $n<\omega$,

$$
\max \left\{d\left(x_{n}, x_{n+1}\right), d\left(x_{n+1}, x_{n}\right)\right\}<2^{-(n+1)},
$$

então $\left\langle x_{n}\right\rangle_{n<\omega}$ é convergente com respeito a $\tau_{d}$.

Teorema 1.2.25. Dados um espaço pré-métrico $\langle X, d\rangle$ e um $A \subseteq X$, considere as seguintes asserções:

(a) $d_{A}$ é completa.

(b) A é um sequencialmente fechado em $\left\langle X, \tau_{d}\right\rangle$.

(c) A é um fechado em $\left\langle X, \tau_{d}\right\rangle$.

Então:

(i) Se d for completa, então

(1) (b) implica (a).

(2) (c) implica (a).

(ii) Se d for uma métrica sobre X, então

(1) (a) implica (b).

(2) sob $\mathbf{A C}_{\omega},(a)$ implica (c). 
Teorema 1.2.26. Dados espaços pré-métricos $\langle X, d\rangle$ e $\left\langle X^{\prime}, d^{\prime}\right\rangle$ tais que $X$ e $X^{\prime}$ são não vazios, uma função uniformemente contínua $\varphi: X \rightarrow X^{\prime}$ segundo d e $d^{\prime}$ e uma sequência s em $X$, considere as seguintes asserções:

(a) A sequência s é de Cauchy segundo d.

(b) A sequência $\varphi$ o s é de Cauchy segundo d'.

(c) d é completa.

(d) d'é completa.

Então:

(i) (a) implica (b).

Suponha que $\varphi$ preserve distância segundo $d$ e d'. Então:

(ii) (a) é equivalente a (b).

Suponha que exista uma sobrejeção de X em $X^{\prime}$ que preserva distância segundo d e d'. Então:

(iii) (d) implica (c).

(iv) Sob $\mathbf{A C}_{\omega}$, (c) é equivalente a (d).

Suponha que d seja uma pseudométrica sobre X e considere a seguinte asserção:

(e) $d / R^{d}$ é completa.

Então:

(v) (e) implica (c).

(vi) Sob $\mathbf{A C}_{\omega},\left(\right.$ c) é equivalente a (e). ${ }^{s}$

No que segue, é introduzida a definição de pseudonorma sobre um espaço vetorial sobre algum subcorpo de $\mathbb{C}$ e são apresentados comentários referentes a essa definição e estabelecidos resultados análogos a outros já apresentados nesta seção.

${ }^{8}$ É interessante destacar que, em $\mathbf{Z F}, \mathbf{A C}{ }_{\omega}$ é equivalente à asserção para todo espaço pseudométrico $\langle X, d\rangle$, dé completa se, e somente se, $d / R^{d}$ for completa, como está afirmado na Proposição 3.1 de [Gut2008] e no Teorema 5 de [HK2015]. 
Definição 1.2.27. Seja $\mathbb{K}$ um subcorpo de $\mathbb{C}$. Sejam $X$ um espaço vetorial sobre $\mathbb{K}$ e $\|\cdot\|: X \rightarrow \mathbb{R}$ uma função. Considere as seguintes condições:

(a) (não negatividade) Para todo $x \in X, 0 \leqslant\|x\|$.

(b) (positividade definida) Para todo $x \in X \backslash\{0\}, 0<\|x\|$.

(c) (subaditividade) Para quaisquer $x, y \in X,\|x+y\| \leqslant\|x\|+\|y\|$.

(d) (condição não arquimediana) Para quaisquer $x, y \in X$,

$$
\|x+y\| \leqslant \max \{\|x\|,\|y\|\}
$$

Denote por $|\cdot|_{\mathbb{C}}$ a função módulo dos números complexos (i.e., a função de $\mathbb{C}$ em $\mathbb{R}$ que é definida pondo-se, para quaisquer $x, y \in \mathbb{R}$,

$$
\left.|x+y i|_{\mathbb{C}}:=\sqrt{x^{2}+y^{2}}\right)
$$

Considere a seguinte condição:

(e) (homogeneidade absoluta) Para todo $x \in X$ e todo $\lambda \in \mathbb{K},\|\lambda x\|=|\lambda|_{\mathbb{C}}\|x\|$.

Diremos que:

(i) $\|\cdot\|$ é uma pseudonorma sobre $X$ se valerem as condições $(a),(c)$ e $(e)$. Se $\|\cdot\|$ for uma pseudonorma sobre $X$, diremos que o par $\langle X,\|\cdot\|\rangle$ é um espaço pseudonormado (sobre $\mathbb{K}$ ).

(ii) $\|\cdot\|$ é uma ultrapseudonorma sobre $X$ se valerem as condições $(a),(d)$ e $(e)$. Se $\|\cdot\|$ for uma ultrapseudonorma sobre $X$, diremos que o par $\langle X,\|\cdot\|\rangle$ é um espaço ultrapseudonormado (sobre $\mathbb{K}$ ).

(iii) $\|\cdot\|$ é uma norma sobre $X$ se $\|\cdot\|$ for uma pseudonorma sobre $X$ e valer a condição (b). Se $\|\cdot\|$ for uma norma sobre $X$, diremos que o par $\langle X,\|\cdot\|\rangle$ é um espaço normado (sobre $\mathbb{K}$ ).

(iv) $\|\cdot\|$ é uma ultranorma sobre $X$ se $\|\cdot\|$ for uma ultrapseudonorma sobre $X$ e valer a condição $(b)$. Se $\|\cdot\|$ for uma ultranorma sobre $X$, diremos que o par $\langle X,\|\cdot\|\rangle$ é um espaço ultranormado (sobre $\mathbb{K}$ ).

Suponha que $\|\cdot\|$ seja uma pseudonorma sobre $X$. Para qualquer $x \in X$, o número real $\|x\|$ será chamado de o comprimento de $x$ (segundo $\|\cdot\|$ ). 
Comentário 1.2.28. Dados um subcorpo $\mathbb{K}$ de $\mathbb{C}$, um espaço vetorial $X$ sobre $\mathbb{K}$ e uma função $\|\cdot\|: X \rightarrow \mathbb{R}$, note que:

(i) (1) Se $\|\cdot\|$ satisfizer a condição de positividade definida, então $\|\cdot\|^{-1}[\{0\}] \subseteq\{0\}$.

(2) Se $\|\cdot\|$ satisfizer a condição de homogeneidade absoluta, então $\|0\|=0$.

Também note que:

(ii) Sobre $X$,

(1) $\|\cdot\|$ é uma norma se, e somente se, $\|\cdot\|$ for uma pseudonorma e satisfizer a condição de positividade definida.

(2) $\|\cdot\|$ é uma uma ultranorma se, e somente se, $\|\cdot\|$ for uma ultrapseudonorma e satisfizer a condição de positividade definida.

É fácil ver que:

(iii) Sobre $X$,

(1) se $\|\cdot\|$ for uma ultrapseudonorma, então $\|\cdot\|$ é uma pseudonorma.

(2) se $\|\cdot\|$ for uma ultranorma, então $\|\cdot\|$ é uma norma.

Mostra-se que:

(iv) (1) $\|\cdot\|$ é uma pseudonorma sobre $X$ se, e somente se, $\|\cdot\|$ satisfizer a condição de subaditividade e de homogeneidade absoluta.

(2) $\|\cdot\|$ é uma norma sobre $X$ se, e somente se, $\|\cdot\|$ satisfizer a condição de positividade definida, de subaditividade e de homogeneidade absoluta.

Agora, considere a função $d_{\|\cdot\|}: X \times X \rightarrow \mathbb{R}$ que é definida pondo-se, para quaisquer $x, y \in X$,

$$
d_{\|\cdot\|}(x, y):=\|x-y\|
$$

Mostra-se facilmente que:

(v) (1) $\|\cdot\|$ ser uma pseudonorma sobre $X$ é condição suficiente para $d_{\|\cdot\|}$ ser uma pseudométrica sobre o conjunto $X$.

(2) $\|\cdot\|$ ser uma ultrapseudonorma sobre $X$ é condição suficiente para $d_{\|\cdot\|}$ ser uma ultrapseudométrica sobre o conjunto $X$. 
Suponha que $\|\cdot\|$ seja uma pseudonorma sobre $X$. A pseudométrica $d_{\|\cdot\|}$ será chamada de a pseudométrica induzida por $\|\cdot\|$ (sobre o conjunto $X$ ). A topologia $\tau_{d_{\|\cdot\|}}$ será chamada de a topologia induzida por $\|\cdot\|$ (sobre o conjunto $X$ ) e denotada por $\tau_{\|\cdot\|}$. Mostra-se que:

(vi) São equivalentes:

(1) $\|\cdot\|$ é uma norma sobre $X$.

(2) $d_{\|\cdot\|}$ é uma métrica sobre o conjunto $X$.

(3) $\{0\}$ é um fechado em $\left\langle X, \tau_{\|\cdot\|}\right\rangle$.

Usando o Comentário 1.2.2 (iv), conclui-se que:

(vii) $\|\cdot\|$ é não expansiva segundo $d_{\|\cdot\|}$ e $d_{2}$.

Denote por $\|\cdot\|_{0, X}$ a função identicamente nula do conjunto $X$ em $\mathbb{R}$ e note que:

(viii) (1) $d_{\|\cdot\|_{0, X}}=d_{0, X}$.

(2) (1) $\|\cdot\|_{0, X}$ é uma ultrapseudonorma sobre $X$.

(2) $\|\cdot\|_{0, X}$ é uma norma sobre $X$ se, e somente se, $X=\{0\}$.

A ultrapseudonorma $\|\cdot\|_{0, X}$ será chamada de a pseudonorma nula de $X$. Considerando $\mathbb{R}$ munido de sua estrutura canônica de espaço vetorial sobre $\mathbb{R}$ e denotando por $\mathbb{R} \mathbb{R}$ tal espaço vetorial, tem-se que:

(ix) $|\cdot|$ é uma norma sobre $\mathbb{R} \mathbb{R}$.

Dado um subespaço vetorial $A$ de $X$, denote por $\|\cdot\|_{A}$ a restrição $\|\cdot\| \uparrow A$ e note que:

(x) (1) $\|\cdot\|_{A}$ é uma pseudonorma sobre $A$.

(2) (1) $d_{\|\cdot\|_{A}}=\left(d_{\|\cdot\|}\right)_{A}$.

(2) $\tau_{\|\cdot\|_{A}}=\left(\tau_{\|\cdot\|}\right)_{A}$.

(3) Se $\|\cdot\|$ for uma norma sobre $X$, então $\|\cdot\|_{A}$ é uma norma sobre $A$.

A pseudonorma $\|\cdot\|_{A}$ será chamada de a pseudonorma induzida por $\|\cdot\|$ sobre $A$.

Definição 1.2.29. Seja $\mathbb{K}$ um subcorpo de $\mathbb{C}$. Sejam $\langle X,\|\cdot\|\rangle$ e $\left\langle X^{\prime},\|\cdot\|^{\prime}\right\rangle$ espaços pseudonormados sobre $\mathbb{K}$ e $\Phi: X \rightarrow X^{\prime}$ uma função. Diremos que:

(i) $\Phi$ é não expansiva segundo $\|\cdot\|$ e $\|\cdot\|^{\prime}$ se valer a seguinte condição: para todo $x \in X,\|\Phi(x)\|^{\prime} \leqslant\|x\|$. 
(ii) $\Phi$ preserva comprimento segundo $\|\cdot\|$ e $\|\cdot\|^{\prime}$ se valer a seguinte condição: para todo $x \in X,\|\Phi(x)\|^{\prime}=\|x\|$.

(iii) $\Phi$ é um monomorfismo isométrico segundo $\|\cdot\|$ e $\|\cdot\|^{\prime}$ se valerem as seguintes condições:

(1) $\Phi$ é um monomorfismo linear.

(2) $\Phi$ preserva comprimento segundo $\|\cdot\|$ e $\|\cdot\|^{\prime}$.

(iv) $\Phi$ é um isomorfismo isométrico segundo $\|\cdot\|$ e $\|\cdot\|^{\prime}$ se valerem as seguintes condições:

(1) $\Phi$ é um isomorfismo linear.

(2) $\Phi$ preserva comprimento segundo $\|\cdot\|$ e $\|\cdot\|^{\prime}$.

Comentário 1.2.30. Dados um subcorpo $\mathbb{K}$ de $\mathbb{C}$, um espaço pseudonormado $\langle X,\|\cdot\|\rangle$ sobre $\mathbb{K}$ e um subconjunto não vazio $A$ de $X$, considere a função $\|\cdot\|^{A}: X \rightarrow \mathbb{R}$ que é definida pondo-se, para todo $x \in X$,

$$
\|x\|^{A}:=\left(d_{\|\cdot\|}\right)^{A}(x)
$$

Dado um $a \in X$, denotaremos por $\|\cdot\|^{a}$ a função $\|\cdot\|^{\{a\}}$. Note que:

(i) (1) (1) Para todo $x \in X,\|x\|^{A}=\inf \{\|x-z\|: z \in A\}$.

(2) Se $0 \in A$, então $\|\cdot\|^{A}$ é não expansiva segundo $\|\cdot\|$ e $|\cdot|$.

(2) (1) Para todo $x \in X,\|x\|^{a}=\|x-a\|$.

(2) $\|\cdot\|^{0}=\|\cdot\|$.

Em virtude do Comentário 1.2.9(ii) (2), tem-se que:

(ii) (1) $\|\cdot\|^{\bar{A}^{\tau} \cdot \cdot \|}=\|\cdot\|^{A}$.

(2) $\|\cdot\|^{\overline{\{a\}}^{\tau_{\|\cdot\|}}}=\|\cdot\|^{a}$.

Em virtude do Comentário 1.2.9(v)(2), tem-se que:

(iii) (1) (1) $\left(\|\cdot\|^{A}\right)^{-1}[\{0\}]=\bar{A}^{\tau_{\|\cdot\|}}$.

(2) $\|\cdot\|^{A}=\|\cdot\|_{0, X}$ se, e somente se, $A$ for um denso em $\left\langle X, \tau_{\|\cdot\|}\right\rangle$.

(2) (1) $\left(\|\cdot\|^{a}\right)^{-1}[\{0\}]=\overline{\{a\}}^{\tau}\|\cdot\|$.

(2) $\|\cdot\|^{a}=\|\cdot\|_{0, X}$ se, e somente se, $\{a\}$ for um denso em $\left\langle X, \tau_{\|\cdot\|}\right\rangle$. 
Suponhamos que $A$ seja um subespaço vetorial de $X$. Mostra-se que:

(iv) (1) $\|\cdot\|^{A}$ é uma pseudonorma sobre $X$.

(2) Se $\|\cdot\|^{A}$ for uma norma sobre $X$, então $A=\{0\}$.

A pseudonorma $\|\cdot\|^{A}$ será chamada de a pseudonorma da distância a $A$ induzida por $\|\cdot\|$ (sobre o conjunto $X$ ). Usando o item (iv) (1), é fácil verificar que:

(v) O conjunto $\bar{A}^{\tau\|\cdot\|}$ é um subespaço vetorial de $X$.

Denote por $\equiv_{A}$ a relação de congruência módulo $A$ sobre $X$ (i.e., a relação de equivalência sobre o conjunto $X$ que é definida pela seguinte sentença:

$$
\text { para quaisquer } \left.x, y \in X, x \equiv_{A} y \text { se, e só se, } x-y \in A\right) \text {. }
$$

É fácil concluir que:

(vi) (1) Para todo $x \in X,[x]_{\equiv_{A}}=x+A$.

(2) $\equiv_{\bar{A}}^{\tau_{\|\cdot\|}}=R^{d_{\|\cdot\|^{A}}}$.

Mostra-se que:

(vii) (1) Para quaisquer $x, y \in X$, se $x \equiv_{A} y$, então $\|x\|^{A}=\|y\|^{A}$.

(2) $d_{\|\cdot\|^{A}}$ é invariante por sucessão segundo $\equiv_{A}$.

Considere o conjunto quociente $X / \equiv_{A}$ munido de sua estrutura canônica de espaço vetorial sobre $\mathbb{K}$. Tal espaço vetorial será chamado de o espaço quociente de $X$ por $A$ e denotado por $X / A$. Em virtude dos itens (vi) (1) e (vii) (1), pode-se considerar a função $\|\cdot\| / A: X / \equiv_{A} \rightarrow \mathbb{R}$ que é definida pondo-se, para todo $x \in X$,

$$
\|x+A\| / A:=\|x\|^{A} .
$$

Usando o item (vi), conclui-se que:

(viii) (1) $d_{\|\cdot\|_{A}}=d_{\|\cdot\|^{A}} / \equiv_{A}$.

(2) $d_{\|\cdot\| / \bar{A}^{\tau_{\|\cdot\|}}}=d_{\|\cdot\|^{A}} / R^{d_{\|\cdot\|^{A}}}$.

Mostra-se que:

(ix) $\|\cdot\| / A$ é uma pseudonorma sobre $X / A$.

(x) São equivalentes: 
(1) $\|\cdot\| / A$ é uma norma sobre $X / A$.

(2) $d_{\|\cdot\|^{A}} / \equiv_{A}$ é uma métrica sobre $X / \equiv_{A}$.

(3) $A$ é um fechado em $\left\langle X, \tau_{\|\cdot\|}\right\rangle$.

Caso $A$ seja um fechado em $\left\langle X, \tau_{\|\cdot\|}\right\rangle$, a função $\|\cdot\| / A$ será chamada de a norma quociente induzida por $\|\cdot\|$ sobre $X / A$. Neste caso, o par $\langle X / A,\|\cdot\| / A\rangle$ será chamado de o espaço quociente de $\langle X,\|\cdot\|\rangle$ por $A$ e denotado por $\langle X,\|\cdot\|\rangle / A$.

Note que não vale em geral a recíproca da implicação nos itens (iv) (2) e (vii) (1) do Comentário 1.2.30, mesmo que se considere, respectivamente, ultrapseudonormas e subespaços vetoriais fechados. De fato: considere a potência cartesiana $\mathbb{R}^{2}$ munida de sua estrutura canônica de espaço vetorial sobre $\mathbb{R}$ e denote por $\mathbb{R}^{2}$ tal espaço vetorial. Sabe-se que, sobre $\mathbb{R}^{2}$, a ultrapseudonorma $\|\cdot\|_{0, \mathbb{R}^{2}}$ não é uma norma (cf. Comentário 1.2.28(viii) (2) (2)). Contudo, $\left(\|\cdot\|_{0, \mathbb{R}^{2}}\right)^{0}=\|\cdot\|_{0, \mathbb{R}^{2}}$ (cf. Comentário 1.2.30(i) (2) (2)). Além disso, note que o produto cartesiano $\mathbb{R} \times\{0\}$ é um subespaço vetorial de $\mathbb{R}^{2}$. Denote por $\|\cdot\|_{2}$ a norma euclidiana sobre $\mathbb{R}^{2}$ (i.e., a função de $\mathbb{R}^{2}$ em $\mathbb{R}$ que é definida pondo-se, para quaisquer $x, y \in \mathbb{R}$,

$$
\left.\|\langle x, y\rangle\|_{2}:=\sqrt{x^{2}+y^{2}}\right)
$$

É fácil ver que, para quaisquer $x, y \in \mathbb{R}$, se $\langle x, y\rangle \notin \mathbb{R} \times\{0\}$, então

$$
|y| \in \mathbb{R}^{+} \text {e } B_{d_{\|\cdot\|_{2}}}(\langle x, y\rangle,|y|) \subseteq \mathbb{R}^{2} \backslash(\mathbb{R} \times\{0\}) .
$$

Então, $\mathbb{R} \times\{0\}$ é um fechado em $\left\langle\mathbb{R}^{2}, \tau_{\|\cdot\|_{2}}\right\rangle$. Como $\left(\|\cdot\|_{2}\right)^{\mathbb{R} \times\{0\}}$ satisfaz a condição de homogeneidade absoluta (cf. Comentário 1.2.30 (iv) (1)), tem-se que:

$$
\left(\|\langle 0,-1\rangle\|_{2}\right)^{\mathbb{R} \times\{0\}}=\left(\|-\langle 0,1\rangle\|_{2}\right)^{\mathbb{R} \times\{0\}}=\left(\|\langle 0,1\rangle\|_{2}\right)^{\mathbb{R} \times\{0\}} .
$$

Contudo, já que $\langle 0,-2\rangle \notin \mathbb{R} \times\{0\}$, tem-se que $\langle 0,-1\rangle \equiv_{\mathbb{R} \times\{0\}}\langle 0,1\rangle$.

O teorema a seguir é uma generalização nossa de um resultado análogo ao já citado Primeiro Teorema do Isomorfismo para álgebras universais e que é utilizado implicitamente na segunda parte da prova da Proposição 2.1 de [S1978].

Teorema 1.2.31. Seja $\mathbb{K}$ um subcorpo de $\mathbb{C}$. Sejam $\langle X,\|\cdot\|\rangle$ e $\left\langle X^{\prime},\|\cdot\|^{\prime}\right\rangle$ espaços pseudonormados sobre $\mathbb{K}$, A um subespaço vetorial de $X$ e $\Phi: X \rightarrow X^{\prime}$ uma função tais que $\Phi$ preserva comprimento segundo $\|\cdot\|^{A}$ e $\|\cdot\|^{\prime}$ e $\left(\|\cdot\|^{\prime}\right)_{\mathrm{im}(\Phi)}$ é uma norma sobre $\mathrm{im}(\Phi)$. Então:

(i) Se $\Phi$ for uma transformação linear, então existe um único um monomorfismo isométrico $\Psi: X / \bar{A}^{\tau_{\|\cdot\|}} \rightarrow X^{\prime}$ segundo $\|\cdot\| / \bar{A}^{\tau_{\|\cdot\|}}$ e $\|\cdot\|^{\prime}$ tal que, para todo $x \in X$,

$$
\Psi\left(x+\bar{A}^{\tau_{\|\cdot\|}}\right)=\Phi(x) \text {. }
$$




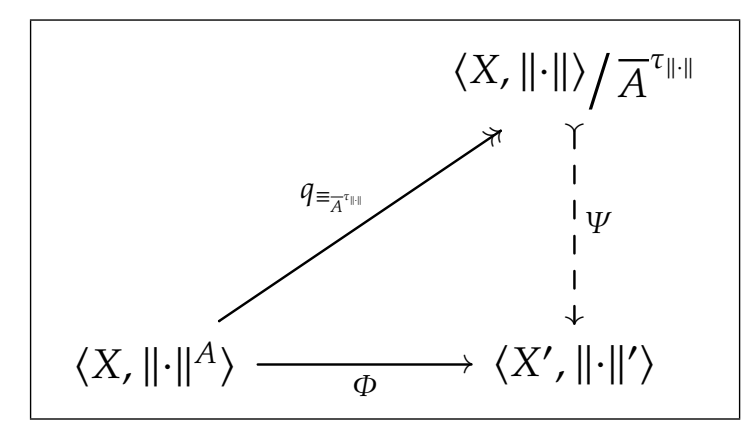

Diagrama i.2

(ii) Se $\Phi$ for um epimorfismo linear, então

(1) $\|\cdot\|^{\prime}$ é uma norma sobre $X^{\prime}$.

(2) $\langle X,\|\cdot\|\rangle / \bar{A}^{\tau_{\|\cdot\|}}$ é isometricamente isomorfo a $\left\langle X^{\prime},\|\cdot\|^{\prime}\right\rangle$.

Definição 1.2.32. Seja $\mathbb{K}$ um subcorpo de $\mathbb{C}$. Seja $\langle X,\|\cdot\|\rangle$ um espaço pseudonormado sobre $\mathbb{K}$. Diremos que:

(i) $\|\cdot\|$ é completa se $d_{\|\cdot\|}$ for completa. Se $\|\cdot\|$ for completa, diremos que $\langle X,\|\cdot\|\rangle$ é completo.

(ii) $\langle X,\|\cdot\|\rangle$ é de Banach se valerem as seguintes condições:

(1) $\|\cdot\|$ é uma norma sobre $X$.

(2) $\|\cdot\|$ é completa.

Uma consequência imediata do Comentário 1.2.28 (x) (2) (1) e do Teorema 1.2.25 é o seguinte

Corolário 1.2.33. Dados um subcorpo $\mathbb{K}$ de $\mathbb{C}$, um espaço pseudonormado $\langle X,\|\cdot\|\rangle$ sobre $\mathbb{K}$ e um subespaço vetorial $A$ de $X$, considere as seguintes asserções:

(a) $\|\cdot\|_{A}$ é completa.

(b) A é um sequencialmente fechado em $\left\langle X, \tau_{\|\cdot\|}\right\rangle$.

(c) A é um fechado em $\left\langle X, \tau_{\|\cdot\|}\right\rangle$.

Então:

(i) Se $\|\cdot\|$ for completa, então 
(1) (b) implica (a).

(2) (c) implica (a).

(ii) Se $\|\cdot\|$ for uma norma sobre $X$, então

(1) (a) implica (b).

(2) sob $\mathbf{A C}_{\omega},(a)$ implica (c).

Definição 1.2.34. Sejam $\langle\mathbb{K}, \sigma\rangle$ um corpo topológico, $X$ um espaço vetorial sobre o corpo $\mathbb{K}$ e $\tau$ uma topologia sobre o conjunto $X$. Diremos que o par $\langle X, \tau\rangle$ é um espaço vetorial topológico sobre $\langle\mathbb{K}, \sigma\rangle$ se valerem as seguintes condições:

(i) A adição sobre $X$ é contínua com respeito a $\tau \otimes \tau$ e $\tau$.

(ii) A multiplicação por escalar de $\mathbb{K}$ sobre $X$ é contínua com respeito a $\sigma \otimes \tau$ e $\tau$.

Comentário 1.2.35. Dado um subcorpo $\mathbb{K}$ de $\mathbb{C}$, considere $\mathbb{C}$ munido de sua estrutura canônica de espaço vetorial sobre $\mathbb{K}$ e denote por ${ }_{K} \mathbb{C}$ tal espaço vetorial. Note que:

(i) (1) $\mathbb{K}$ é um subespaço vetorial de $\mathbb{K} \mathbb{C}$.

(2) $|\cdot|_{\mathbb{C}}$ é uma norma sobre ${ }_{\mathbb{K}} \mathbb{C}$.

Denote por $\mathbb{K}_{\mathbb{K}} \mathbb{K}$ o conjunto $\mathbb{K}$ munido de sua estrutura de subespaço vetorial de $\mathbb{K}_{\mathbb{C}} \mathbb{\text { . }}$ Em virtude do item (i) (2) e do Comentário 1.2.28 (x) (3), tem-se que:

(ii) $\left(|\cdot|_{\mathbb{C}}\right)_{\mathbb{K}}$ é uma norma sobre $\mathbb{K} \mathbb{K}$.

A norma $\left(|\cdot|_{\mathbb{C}}\right)_{\mathbb{K}}$ será denotada por $|\cdot|_{\mathbb{K}}$. A métrica $d_{|\cdot|_{K}}$ e a topologia $\tau_{|\cdot|_{K}}$ serão denotadas, respectivamente, por $d_{\mathbb{K}}$ e por $\tau_{\mathbb{K}}$. É imediato concluir que:

(iii) Se $\mathbb{K}$ for um subcorpo de $\mathbb{R}$, então

(1) (1) $|\cdot|_{\mathbb{K}}=|\cdot| \uparrow \mathbb{K} \times \mathbb{K}$.

(2) $|\cdot|_{\mathbb{R}}=|\cdot|$.

(2) $(1) d_{\mathbb{K}}=\left(d_{2}\right)_{\mathbb{K}}$.

(2) $d_{\mathbb{R}}=d_{2}$.

(3) (1) $\tau_{\mathbb{K}}=\left(\tau_{2}\right)_{\mathbb{K}}$.

(2) $\tau_{\mathbb{R}}=\tau_{2}$. 
Mostra-se que:

(iv) $\mathrm{O} \operatorname{par}\left\langle\mathbb{K}, \tau_{\mathbb{K}}\right\rangle$ é um corpo topológico.

(v) Para todo espaço pseudonormado $\langle X,\|\cdot\|\rangle$ sobre $\mathbb{K}$, o par $\left\langle X, \tau_{\|\cdot\|}\right\rangle$ é um espaço vetorial topológico sobre $\left\langle\mathbb{K}, \tau_{\mathbb{K}}\right\rangle$.

Encerraremos esta seção com a apresentação das definições de espaços de funções contínuas e de resultados já conhecidos sobre esses espaços normados.

Comentário 1.2.36 ([Rud1987, cap. 3], [Meg1998, cap. 1, seç. 2]). Dados um conjunto X e uma topologia $\tau$ sobre $X$, considere os seguintes conjuntos:

$$
\begin{aligned}
C(X, \tau) & :=\left\{\zeta \in \mathbb{R}^{X}: \zeta \text { é contínua com respeito a } \tau \text { e } \tau_{2}\right\} \\
& \text { e } \\
C_{0}(X, \tau) & :=\left\{\zeta \in C(X, \tau): \forall \varepsilon \in \mathbb{R}^{+}\left(\left\langle|\zeta|^{-1}\left[\left[\varepsilon,+\infty[], \tau_{|\zeta|^{-1}[[\varepsilon,+\infty[]}\right\rangle \text { é compacto }\right)\right\} .\right.\right.
\end{aligned}
$$

Denote por $\operatorname{Bor}(\mathbb{R})$ a $\sigma$-álgebra de Borel sobre $\left\langle\mathbb{R}, \tau_{2}\right\rangle$ (i.e., a $\sigma$-álgebra sobre $\mathbb{R}$ que é gerada por $\tau_{2}$ ). Dadas uma $\sigma$-álgebra $\Sigma$ sobre $X$ e uma medida não negativa $\mu$ definida em $\Sigma$ tal que $\mu$ é $\sigma$-aditiva, também considere os seguintes conjuntos:

$$
\begin{aligned}
\mathscr{M}(X, \Sigma) & :=\left\{\zeta \in \mathbb{R}^{X}: \zeta \text { é mensurável com respeito a } \Sigma \text { e Bor }(\mathbb{R})\right\} \\
& \text { e } \\
\mathscr{L}_{\infty}(X, \Sigma, \mu) & :=\left\{\zeta \in \mathscr{M}(X, \Sigma): \exists \delta \in \mathbb{R}^{+}\left(\mu\left(|\zeta|^{-1}[] \delta,+\infty[]\right)=0\right)\right\} .
\end{aligned}
$$

É claro que:

(i) (1) $C(X, \mathcal{P}(X))=\mathbb{R}^{X}$.

(2) $\mathscr{M}(X, \mathcal{P}(X))=\mathbb{R}^{X}$.

Denote por \# a medida de contagem definida em $\mathcal{P}(X)$. Os conjuntos $C_{0}(X, \mathcal{P}(X))$ e $\mathscr{L}_{\infty}\left(X, \mathcal{P}(X)\right.$,\#) serão denotados, respectivamente, por $c_{0}(X)$ e por $\ell_{\infty}(X)$. Os conjuntos $c_{0}(\omega)$ e $\ell_{\infty}(\omega)$ serão denotados, respectivamente, por $c_{0}$ e por $\ell_{\infty}$. Com o uso do item $(i)$ acima, dos itens (i) (1) e (i) (5) da Proposição 1.1.17, dos itens (i) e (iv) da Proposição 1.2.19 e da Proposição 1.2.15 (v), é corriqueiro mostrar que:

(ii) (1) (1) $c_{0}(X)=\left\{\zeta \in \mathbb{R}^{X}: \forall \varepsilon \in \mathbb{R}^{+}\left(|\zeta|^{-1}[[\varepsilon,+\infty[]\right.\right.$ é finito $)\}$.

(2) $c_{0}=\left\{s \in \mathbb{R}^{\omega}: s\right.$ converge para 0 com respeito a $\left.\tau_{2}\right\}$.

(2) $\ell_{\infty}(X)=\left\{\zeta \in \mathbb{R}^{X}: \zeta\right.$ é limitada segundo $\left.d_{2}\right\}$.

Considere o conjunto $C_{b}(X, \tau):=C(X, \tau) \cap \ell_{\infty}(X)$. Usando o item (i) (1), tem-se que: 
(iii) $C_{b}(X, \mathcal{P}(X))=\ell_{\infty}(X)$.

Com a utilização do resultado que afirma que a imagem por uma função contínua de um espaço topológico compacto é um subespaço topológico compacto do espaço contradomínio dessa função e da Proposição 1.2.15 (vi) e usando o item (ii) (2), conclui-se que:

(iv) Se $\langle X, \tau\rangle$ for compacto, então

(1) $C(X, \tau) \subseteq \ell_{\infty}(X)$.

(2) $C(X, \tau)=C_{b}(X, \tau)$.

Mostra-se que:

(v) (1) $C_{0}(X, \tau) \subseteq C_{b}(X, \tau)$.

(2) $c_{0}(X) \subseteq \ell_{\infty}(X)$.

Agora, suponha que $X$ seja não vazio. Note que a potência cartesiana $\mathbb{R}^{X}$ é um espaço vetorial sobre $\mathbb{R}$ quando munida da adição e da multiplicação por escalar de $\mathbb{R}$ que são definidas coordenada a coordenada - e o seu vetor nulo é a função identicamente nula de $X$ em $\mathbb{R}$. Denote por ${ }_{\mathbb{R}} \mathbb{R}^{X}$ tal espaço vetorial. Prova-se que $C_{0}(X, \tau), \mathscr{L}_{\infty}(X, \Sigma, \mu)$ e $C_{b}(X, \tau)$ são subespaços vetoriais de $\mathbb{R}_{\mathbb{R}} \mathbb{R}$. Denote por ${ }_{\mathbb{R}} C_{0}(X, \tau)$, por $\mathbb{R}_{\mathbb{R}} \mathscr{L}_{\infty}(X, \Sigma, \mu)$ e por ${ }_{\mathbb{R}} C_{b}(X, \tau)$, respectivamente, os conjuntos $C_{0}(X, \tau), \mathscr{L}_{\infty}(X, \Sigma, \mu)$ e $C_{b}(X, \tau)$ munidos de suas estruturas de subespaço vetorial de $\mathbb{R}^{X}$. É claro que:

(vi) $C_{b}(X, \tau)$ é um subespaço vetorial de $\mathbb{R}_{\mathbb{R}}(X)$.

Usando o item (v), tem-se que:

(vii) (1) $C_{0}(X, \tau)$ é um subespaço vetorial de ${ }_{\mathbb{R}} C_{b}(X, \tau)$.

(2) $c_{0}(X)$ é um subespaço vetorial de ${ }_{\mathbb{R}} \ell_{\infty}(X)$.

Considere a função $\|\cdot\|_{\infty, \mu}: \mathscr{L}_{\infty}(X, \Sigma, \mu) \rightarrow \mathbb{R}$ que é definida pondo-se, para toda função $\xi \in \mathscr{L}_{\infty}(X, \Sigma, \mu)$,

$$
\|\xi\|_{\infty, \mu}:=\inf \left\{\delta \in \mathbb{R}^{+}: \mu\left(|\xi|^{-1}[] \delta,+\infty[]\right)=0\right\}
$$

Prova-se que $\|\cdot\|_{\infty, \mu}$ é uma pseudonorma sobre $\mathbb{R}_{\infty} \mathscr{L}_{\infty}(X, \Sigma, \mu)$ que é completa. Denote

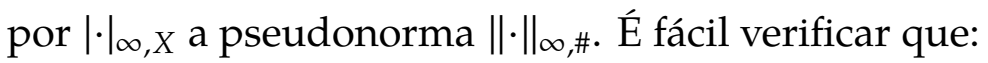

(viii) (1) (1) Para todo $\xi \in \ell_{\infty}(X),|\xi|_{\infty, X}=\sup \{|\xi(x)|: x \in X\}$.

(2) $|\cdot|_{\infty, X}$ é uma norma sobre ${ }_{\mathbb{R}} \ell_{\infty}(X)$. 
(2) O espaço normado $\left\langle\ell_{\infty}(X),|\cdot|_{\infty, X}\right\rangle$ é de Banach.

Em virtude do item (viii) (1) (2) e do Comentário 1.2.28 (x) (3), tem-se que:

(ix) (1) $\left(|\cdot|_{\infty, X}\right)_{C_{b}(X, \tau)}$ é uma norma sobre $\mathbb{R}_{\mathbb{R}} C_{b}(X, \tau)$.

(2) $\left(|\cdot|_{\infty, X}\right)_{\mathcal{C}_{0}(X)}$ é uma norma sobre $\mathbb{R}_{\mathbb{R}} \mathcal{C}_{0}(X)$.

Prova-se que $C_{b}(X, \tau)$ é um sequencialmente fechado em $\left\langle\ell_{\infty}(X), \tau_{|\cdot|_{\infty, X}}\right\rangle$ e que $c_{0}(X)$ é um fechado em $\left\langle\ell_{\infty}(X), \tau_{|\cdot|_{\infty, X}}\right\rangle$. Usando o Corolário 1.2.33(i), tem-se que:

(x) (1) O espaço normado $\left\langle C_{b}(X, \tau),\left(|\cdot|_{\infty, X}\right)_{C_{b}(X, \tau)}\right\rangle$ é de Banach.

(2) O espaço normado $\left\langle c_{0}(X),\left(|\cdot|_{\infty, X}\right)_{c_{0}(X)}\right\rangle$ é de Banach.

Comentário 1.2.37. Dados espaços topológicos $\langle X, \tau\rangle$ e $\left\langle X^{\prime}, \tau^{\prime}\right\rangle$ e um espaço pré-métrico $\langle M, d\rangle$, considere os seguintes conjuntos:

$$
\begin{aligned}
& C\left(X, \tau, X^{\prime}, \tau^{\prime}\right):=\left\{\zeta \in\left(X^{\prime}\right)^{X}: \zeta \text { é contínua com respeito a } \tau \text { e } \tau^{\prime}\right\} \\
& \text { e } \\
& C(X, \tau, M, d):=C\left(X, \tau, M, \tau_{d}\right) .
\end{aligned}
$$

Também considere os seguintes conjuntos:

$$
\begin{aligned}
\ell_{\infty}(X, M, d) & :=\left\{\zeta \in M^{X}: \zeta \text { é limitada segundo } d\right\} \\
& \text { e } \\
C_{b}(X, \tau, M, d) & :=C(X, \tau, M, d) \cap \ell_{\infty}(X, M, d) .
\end{aligned}
$$

É claro que:

(i) (1) $C\left(X, \tau, \mathbb{R}, d_{2}\right)=C(X, \tau)$.

(2) (1) $\ell_{\infty}\left(X, \mathbb{R}, d_{2}\right)=\ell_{\infty}(X)$.

(2) $C_{b}\left(X, \tau, \mathbb{R}, d_{2}\right)=C_{b}(X, \tau)$.

Em virtude da Proposição 1.2.16(i), dada uma função contínua $\gamma: X \rightarrow X^{\prime}$ com respeito a $\tau$ e $\tau^{\prime}$, pode-se considerar a função $\gamma_{d, \circ}: C_{b}\left(X^{\prime}, \tau^{\prime}, M, d\right) \rightarrow C_{b}(X, \tau, M, d)$ que é definida pondo-se, para toda função $\xi \in C_{b}\left(X^{\prime}, \tau^{\prime}, M, d\right)$,

$$
\gamma_{d, \circ}(\xi):=\xi \circ \gamma
$$

Mostra-se que:

(ii) (1) Se $\gamma$ for sobrejetora, então $\gamma_{d, o}$ é injetora. 
(2) Se $\gamma$ for um homeomorfismo com respeito a $\tau$ e $\tau^{\prime}$, então

(1) $\gamma_{d, o}$ é bijetora e

(2) $\left(\gamma_{d, o}\right)^{-1}=\left(\gamma^{-1}\right)_{d, o}$.

Suponha que $d$ seja uma hemimétrica sobre $X$ e que $X$ seja não vazio. Em virtude da Proposição 1.2.16 (ii), pode-se considerar a função

$$
d_{\infty, X}: C_{b}(X, \tau, M, d) \times C_{b}(X, \tau, M, d) \rightarrow \mathbb{R}
$$

que é definida pondo-se, para quaisquer $\xi, \xi^{\prime} \in C_{b}(X, \tau, M, d)$,

$$
d_{\infty, X}\left(\xi, \xi^{\prime}\right):=\sup \left\{d\left(\xi(x), \xi^{\prime}(x)\right): x \in X\right\}
$$

Mostra-se que:

(iii) (1) $d_{\infty, X}$ é uma hemimétrica sobre $C_{b}(X, \tau, M, d)$.

(2) $d$ ser uma pseudométrica sobre $X$ é condição suficiente para $d_{\infty, X}$ ser uma pseudométrica sobre $C_{b}(X, \tau, M, d)$.

(3) d ser uma métrica sobre $X$ é condição suficiente para $d_{\infty, X}$ ser uma métrica sobre $C_{b}(X, \tau, M, d)$.

Suponha que $X^{\prime}$ seja não vazio. Mostra-se também que:

(iv) (1) Se $\gamma$ for uma sobrejeção, então $\gamma_{d, o}$ é uma imersão isométrica segundo $d_{\infty, X^{\prime}}$ e $d_{\infty, X}$.

(2) $\gamma$ ser um homeomorfismo com respeito a $\tau$ e $\tau^{\prime}$ é condição suficiente para $\gamma_{d, \circ}$ ser uma isometria segundo $d_{\infty, X^{\prime}}$ e $d_{\infty, X}$.

Agora, dados um subcorpo $\mathbb{K}$ de $\mathbb{C}$ e um espaço pseudonormado $\langle N,\|\cdot\|\rangle$ sobre $\mathbb{K}$, os conjuntos $\ell_{\infty}\left(X, N, d_{\|\cdot\| \|}\right)$ e $C\left(X, \tau, N, d_{\|\cdot\|}\right)$ serão denotados, respectivamente, por $\ell_{\infty}(X, N,\|\cdot\|)$ e por $C(X, \tau, N,\|\cdot\|)$. O conjunto $C_{b}\left(X, \tau, N, d_{\|\cdot\|}\right)$ e a função $\gamma_{d_{\|\cdot\|,} \text { serão }}$ denotados, respectivamente, por $C_{b}(X, \tau, N,\|\cdot\|)$ e por $\gamma_{\|\cdot\|, \circ}$ É claro que:

(v) (1) $C(X, \tau, \mathbb{R},|\cdot|)=C(X, \tau)$.

(2) (1) $\ell_{\infty}(X, \mathbb{R},|\cdot|)=\ell_{\infty}(X)$.

(2) $C_{b}(X, \tau, \mathbb{R},|\cdot|)=C_{b}(X, \tau)$.

Considere os conjuntos $C_{b}(X, \tau, N,\|\cdot\|)$ e $C_{b}\left(X^{\prime}, \tau^{\prime}, N,\|\cdot\|\right)$ munidos de suas estruturas de subespaço vetorial de $\mathbb{R}^{X}$ (v. Comentário 1.2.36). É fácil ver que: 
(vi) $\gamma_{\|\cdot\|, o}$ é uma transformação linear.

Considere a função $\|\cdot\|_{\infty, X}: C_{b}(X, \tau, N,\|\cdot\|) \rightarrow \mathbb{R}$ que é definida pondo-se, para todo $\xi \in C_{b}(X, \tau, N,\|\cdot\|)$,

$$
\|\xi\|_{\infty, X}:=\sup \{\|\xi(x)\|: x \in X\}
$$

Note que:

(vii) $d_{\|\cdot\|_{\infty, X}}=\left(d_{\|\cdot\|}\right)_{\infty, X}$

Mostra-se que:

(viii) (1) $\|\cdot\|_{\infty, X}$ é uma pseudonorma sobre $C_{b}(X, \tau, N,\|\cdot\|)$.

(2) $\|\cdot\|$ ser uma norma sobre $X$ é condição suficiente para $\|\cdot\|_{\infty, X}$ ser uma norma sobre $C_{b}(X, \tau, N,\|\cdot\|)$.

Usando o item (iv), conclui-se que:

(ix) (1) Se $\gamma$ for uma sobrejeção, então $\gamma_{\|\cdot\|, o}$ é um monomorfismo isométrico segundo $\|\cdot\|_{\infty, X^{\prime}}$ e $\|\cdot\|_{\infty, X}$

(2) $\gamma$ ser um homeomorfismo com respeito a $\tau$ e $\tau^{\prime}$ é condição suficiente para $\gamma_{\|\cdot\|, \circ}$ ser um isomorfismo isométrico segundo $\|\cdot\|_{\infty, X^{\prime}}$ e $\|\cdot\|_{\infty, X} \cdot$ 


\section{CAPÍTULO 2}

\section{Ultraprodutos métricos e normados}

Neste capítulo, são estabelecidas, em ZF ou em particulares extensões próprias de ZF, generalizações de definições e resultados que são encontrados nas referências adotadas sobre ultraprodutos de espaços métricos ou sobre ultraprodutos de espaços normados e de Banach. Para estabelecer essas generalizações, é introduzida a definição de conjunto finitamente distal com respeito a uma família de pré-métricas.

\subsection{Convergência com respeito a filtros}

Nesta seção, são estabelecidas generalizações de definições e resultados clássicos sobre convergência de redes e filtros, que são comumente estabelecidos no contexto ingênuo ${ }^{1}$ dos espaços topológicos.

Definição 2.1.1. Sejam $I$ um conjunto não vazio e $\mathcal{F}$ uma subfamília não vazia de $\mathcal{P}(I)$. Sejam $X$ um conjunto não vazio, $\left\langle x_{i}\right\rangle_{i \in I}$ uma família de pontos de $X, A \subseteq X$ e $a \in X$. Diremos que:

(i) $\left\langle x_{i}\right\rangle_{i \in I}$ está $\mathcal{F}$-eventualmente em $A$ se $\left\{i \in I: x_{i} \in A\right\} \in \mathcal{F}$. Se $\left\langle x_{i}\right\rangle_{i \in I}$ estiver $\mathcal{F}$ eventualmente em $\{a\}$, diremos que $\left\langle x_{i}\right\rangle_{i \in I}$ é $\mathcal{F}$-eventualmente constante de valor $a$.

\footnotetext{
${ }^{1}$ Lembrar que este termo é uma tradução da palavra francesa naïve, que teóricos conjuntistas utilizam com a conotação de algo despreocupado ou pouco cauteloso em relação ao rigor lógico da Teoria Axiomática dos Conjuntos.
} 
(ii) $\left\langle x_{i}\right\rangle_{i \in I}$ é $\mathcal{F}$-eventualmente constante se valer a seguinte condição: existe um $x \in X$ tal que $\left\langle x_{i}\right\rangle_{i \in I}$ é $\mathcal{F}$-eventualmente constante de valor $x$.

(iii) $\left\langle x_{i}\right\rangle_{i \in I}$ está $\mathcal{F}$-frequentemente em $A$ se valer a seguinte condição: não é verdade que $\left\langle x_{i}\right\rangle_{i \in I}$ está $\mathcal{F}$-eventualmente em $X \backslash A$.

(iv) $\left\langle x_{i}\right\rangle_{i \in I}$ é $\mathcal{F}$-universal (em $X$ ) se valer a seguinte condição: para todo $B \subseteq X$, se $\left\langle x_{i}\right\rangle_{i \in I}$ estiver $\mathcal{F}$-frequentemente em $B$, então $\left\langle x_{i}\right\rangle_{i \in I}$ está $\mathcal{F}$-eventualmente em $B$.

Comentário 2.1.2. Dados um conjunto não vazio $I$, subfamílias não vazias $\mathcal{F}$ e $\mathcal{G}$ de $\mathcal{P}(I)$ tais que $\mathcal{F} \subseteq \mathcal{G}$, um conjunto não vazio $X$, uma família $\left\langle x_{i}\right\rangle_{i \in I}$ de pontos de $X$, $A \subseteq X$ e um $a \in X$, é imediato concluir que:

(i) (1) $\left\langle x_{i}\right\rangle_{i \in I}$ estar $\mathcal{F}$-eventualmente em $A$ é condição suficiente para $\left\langle x_{i}\right\rangle_{i \in I}$ estar $\mathcal{G}$-eventualmente em $A$.

(2) $\left\langle x_{i}\right\rangle_{i \in I} \operatorname{ser} \mathcal{F}$-eventualmente constante de valor $a$ é condição suficiente para $\left\langle x_{i}\right\rangle_{i \in I}$ ser $\mathcal{G}$-eventualmente constante de valor $a$.

(3) $\left\langle x_{i}\right\rangle_{i \in I}$ estar $\mathcal{G}$-frequentemente em $A$ é condição suficiente para $\left\langle x_{i}\right\rangle_{i \in I}$ estar $\mathcal{F}$-frequentemente em $A$.

Usando os itens (i) (1) e (i) (3), conclui-se que:

(ii) Se $\left\langle x_{i}\right\rangle_{i \in I}$ for $\mathcal{F}$-universal em $X$, então $\left\langle x_{i}\right\rangle_{i \in I}$ é $\mathcal{G}$-universal em $X$.

Lembre-se que, para cada $i \in I, \mathcal{U}(i)$ denota o ultrafiltro principal sobre $I$ em $i$ (cf. Comentário 1.1.2). Suponha que $\mathcal{F}$ seja um filtro sobre $I$. É fácil verificar que:

(iii) (1) Se $\left\langle x_{i}\right\rangle_{i \in I}$ for constante de valor $a$, então $\left\langle x_{i}\right\rangle_{i \in I}$ é $\mathcal{F}$-eventualmente constante de valor $a$.

(2) Para todo $j \in I$, se $\mathcal{F}=\mathcal{U}(j)$, então $\left\langle x_{i}\right\rangle_{i \in I}$ é $\mathcal{F}$-eventualmente constante de valor $x_{j}$.

(iv) (1) Se $\left\langle x_{i}\right\rangle_{i \in I}$ for $\mathcal{F}$-eventualmente constante, então $\left\langle x_{i}\right\rangle_{i \in I}$ é $\mathcal{F}$-universal em $X$.

(2) Se $\left\langle x_{i}\right\rangle_{i \in I}$ for constante, então $\left\langle x_{i}\right\rangle_{i \in I}$ é $\mathcal{F}$-universal em $X$.

(v) (1) $\operatorname{Se}\left\langle x_{i}\right\rangle_{i \in I}$ estiver $\mathcal{F}$-eventualmente em $A$, então $\left\langle x_{i}\right\rangle_{i \in I}$ está $\mathcal{F}$-frequentemente em $A$.

(2) Se $\mathcal{F}$ for um ultrafiltro sobre $I$, então $\left\langle x_{i}\right\rangle_{i \in I}$ está $\mathcal{F}$-eventualmente em $A$ se, e somente se, $\left\langle x_{i}\right\rangle_{i \in I}$ estiver $\mathcal{F}$-frequentemente em $A$. 
Usando o item (v) (2), conclui-se que:

(vi) Se $\mathcal{F}$ for um ultrafiltro sobre $I$, então $\left\langle x_{i}\right\rangle_{i \in I}$ é $\mathcal{F}$-universal em $X$.

Dados um conjunto não vazio $X^{\prime}$, uma função $\varphi: X \rightarrow X^{\prime}$, um $A^{\prime} \subseteq X^{\prime}$ e um $B \subseteq X$, é corriqueiro mostrar que:

(vii) (1) (1) Se $\varphi[A] \subseteq A^{\prime}$ e $\left\langle x_{i}\right\rangle_{i \in I}$ estiver $\mathcal{F}$-eventualmente em $A$, então a família $\left\langle\varphi\left(x_{i}\right)\right\rangle_{i \in I}$ está $\mathcal{F}$-eventualmente em $A^{\prime}$.

(2) Se $\varphi[X \backslash A] \subseteq X^{\prime} \backslash A^{\prime}$ e a família $\left\langle\varphi\left(x_{i}\right)\right\rangle_{i \in I}$ estiver $\mathcal{F}$-frequentemente em $A^{\prime}$, então $\left\langle x_{i}\right\rangle_{i \in I}$ está $\mathcal{F}$-frequentemente em $A$.

(2) Se $A \subseteq B$, então

(1) $\left\langle x_{i}\right\rangle_{i \in I}$ estar $\mathcal{F}$-eventualmente em $A$ é condição suficiente para $\left\langle x_{i}\right\rangle_{i \in I}$ estar $\mathcal{F}$-eventualmente em $B$.

(2) $\left\langle x_{i}\right\rangle_{i \in I}$ estar $\mathcal{F}$-frequentemente em $A$ é condição suficiente para $\left\langle x_{i}\right\rangle_{i \in I}$ estar $\mathcal{F}$-frequentemente em $B$.

(3) (1) Se $\left\langle x_{i}\right\rangle_{i \in I}$ estiver $\mathcal{F}$-eventualmente em $A$ ou estiver $\mathcal{F}$-eventualmente em $B$, então $\left\langle x_{i}\right\rangle_{i \in I}$ está $\mathcal{F}$-eventualmente em $A \cup B$.

(2) Se $\left\langle x_{i}\right\rangle_{i \in I}$ estiver $\mathcal{F}$-frequentemente em $A$ e estiver $\mathcal{F}$-frequentemente em $B$, então $\left\langle x_{i}\right\rangle_{i \in I}$ está $\mathcal{F}$-frequentemente em $A \cap B$.

É fácil verificar que:

(viii) (1) $\left\langle x_{i}\right\rangle_{i \in I}$ está $\mathcal{F}$-eventualmente em $A \cap B$ se, e somente se, $\left\langle x_{i}\right\rangle_{i \in I}$ estiver $\mathcal{F}$ eventualmente em $A$ e estiver $\mathcal{F}$-eventualmente em $B$.

(2) $\left\langle x_{i}\right\rangle_{i \in I}$ está $\mathcal{F}$-frequentemente em $A \cup B$ se, e somente se, $\left\langle x_{i}\right\rangle_{i \in I}$ estiver $\mathcal{F}$-frequentemente em $A$ ou estiver $\mathcal{F}$-frequentemente em $B$.

Suponha que $\left\{x_{i}: i \in I\right\} \subseteq A$. É imediato concluir que:

(ix) (1) $\left\langle x_{i}\right\rangle_{i \in I}$ está $\mathcal{F}$-eventualmente em $A$.

(2) $\left\langle x_{i}\right\rangle_{i \in I}$ está $\mathcal{F}$-frequentemente em $A$.

Usando os itens (ix)(2), (viii) (3) (2) e (viii) (2) (1), mostra-se facilmente que:

$(x)\left\langle x_{i}\right\rangle_{i \in I}$ é $\mathcal{F}$-universal em $X$ se, e somente se, $\left\langle x_{i}\right\rangle_{i \in I}$ for $\mathcal{F}$-universal em $A$.

O teorema a seguir fornece uma caracterização de maximalidade de filtros por meio da propriedade de ser universal com respeito a esses filtros. 
Teorema 2.1.3. Sejam I um conjunto não vazio e $\mathcal{F}$ um filtro sobre I. Então, são equivalentes:

(i) $\mathcal{F}$ é um ultrafiltro.

(ii) Para todo conjunto não vazio X e toda família $\left\langle x_{i}\right\rangle_{i \in I}$ de pontos de $X,\left\langle x_{i}\right\rangle_{i \in I}$ é $\mathcal{F}$-universal em X.

Prova. $(i) \Longrightarrow($ ii $)$ : É imediato do Comentário 2.1.2(vi).

(ii) $\Longrightarrow(i)$ : Fixe arbitrariamente um $A \subseteq I$. Denote por $\chi_{A}$ a função característica do conjunto $A$ definida em $I$. Assim, tem-se que:

$$
A=\left\{i \in I: \chi_{A}(i) \in\{1\}\right\}=\left\{i \in I: \chi_{A}(i) \in 2 \backslash 1\right\} .
$$

Suponha que $A \notin \mathcal{F}$. Então, $\chi_{A}$ está $\mathcal{F}$-frequentemente no natural 1. Suponha também que (ii) valha. Sendo assim, $\chi_{A}$ é $\mathcal{F}$-universal em $X$. Logo, $\chi_{A}$ é $\mathcal{F}$-eventualmente constante de valor 0 , o que implica que

$$
I \backslash A=\left\{i \in I: \chi_{A}(i) \in 1\right\} \in \mathcal{F} .
$$

Portanto, como $A \subseteq I$ foi fixado arbitrariamente, conclui-se que (i) vale.

Definição 2.1.4. Sejam $I$ um conjunto não vazio e $\mathcal{F}$ uma subfamília não vazia de $\mathcal{P}(I)$. Sejam $\langle X, \tau\rangle$ um espaço topológico tal que $X$ é não vazio, $\left\langle x_{i}\right\rangle_{i \in I}$ uma família de pontos de $X$ e $a \in X$. Diremos que:

(i) $\left\langle x_{i}\right\rangle_{i \in I} \mathcal{F}$-converge para a com respeito a $\tau$ (ou em $\langle X, \tau\rangle$ ) se valer a seguinte condição: para toda vizinhança $V$ de $x$ em $\langle X, \tau\rangle,\left\langle x_{i}\right\rangle_{i \in I}$ está $\mathcal{F}$-eventualmente em $V$. Se $\left\langle x_{i}\right\rangle_{i \in I} \mathcal{F}$-convergir para $a$ com respeito a $\tau$, diremos que $a$ é um $\mathcal{F}$-limite de $\left\langle x_{i}\right\rangle_{i \in I}$ com respeito a $\tau$ (ou em $\langle X, \tau\rangle$ ).

(ii) $\left\langle x_{i}\right\rangle_{i \in I}$ é $\mathcal{F}$-convergente com respeito a $\tau$ (ou em $\langle X, \tau\rangle$ ) se valer a seguinte condição: existe um $x \in X$ tal que $x$ é um $\mathcal{F}$-limite de $\left\langle x_{i}\right\rangle_{i \in I}$ com respeito a $\tau$.

(iii) $\left\langle x_{i}\right\rangle_{i \in I}$ é $\mathcal{F}$-divergente com respeito a $\tau$ se $\left\langle x_{i}\right\rangle_{i \in I}$ não for $\mathcal{F}$-convergente com respeito a $\tau$.

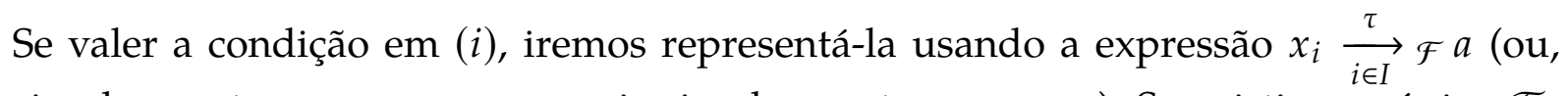
simplesmente, $x_{i} \underset{i \in I}{\longrightarrow} \mathcal{F} a$ ou, mais simplesmente, $x_{i} \rightarrow \mathcal{F} a$ ). Se existir um único $\mathcal{F}$ limite de $\left\langle x_{i}\right\rangle_{i \in I}$ com respeito a $\tau$, iremos denotá-lo por $\mathcal{F}$ - $\lim _{i \in I}^{\tau} x_{i}$ (ou, simplesmente, por $\mathcal{F}-\lim _{i \in I} x_{i}$ ou, mais simplesmente, por $\left.\mathcal{F}-\lim x_{i}\right)$. 
Comentário 2.1.5. Dados um conjunto não vazio $I$, subfamílias não vazias $\mathcal{F}$ e $\mathcal{G}$ de $\mathcal{P}(I)$ tais que $\mathcal{F} \subseteq \mathcal{G}$, um espaço topológico $\langle X, \tau\rangle$ tal que $X$ é não vazio, uma família $\left\langle x_{i}\right\rangle_{i \in I}$ de pontos de $X$ e um $a \in X$, é imediato concluir que:

(i) Se $x_{i} \underset{i \in I}{\stackrel{\tau}{\longrightarrow}} \mathcal{F} a$, então $x_{i} \underset{i \in I}{\stackrel{\tau}{\longrightarrow}} \mathcal{G} a$.

Dado um $A \subseteq X$ tal que $\left\{x_{n}: n<\omega\right\} \subseteq A$, é fácil verificar que:

(ii) Se $a \in A$, então $x_{i} \underset{i \in I}{\stackrel{\tau}{\longrightarrow}} \mathcal{F} a$ se, e somente se, $x_{i} \underset{i \in I}{\stackrel{\tau_{A}}{\longrightarrow}} \mathcal{F} a$.

Suponha que $\mathcal{F}$ seja um filtro sobre $I$. É fácil ver que:

(iii) Se $x_{i} \underset{i \in I}{\stackrel{\tau}{\longrightarrow}} \mathcal{F} a$, então $a \in \bar{A}^{\tau}$.

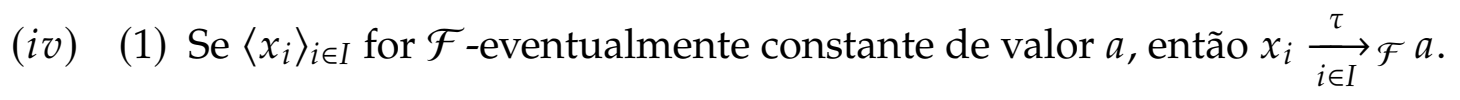

(2) Se $\left\langle x_{i}\right\rangle_{i \in I}$ for constante de valor $a$, então $x_{i} \underset{i \in I}{\stackrel{\tau}{\longrightarrow}} \mathcal{F} a$.

Lembre-se que, para cada $i \in I, \mathcal{U}(i)$ denota o ultrafiltro principal sobre $I$ em $i$ (cf. Comentário 1.1.2). Em virtude do item (iv)(1) e do Comentário 2.1.2(iii)(2), tem-se que:

(v) Para todo $j \in I$, se $\mathcal{F}=\mathcal{U}(j)$, então $x_{i} \underset{i \in I}{\stackrel{\tau}{\longrightarrow}} \mathcal{F} x_{j}$.

Lembre-se que $\mathcal{N}_{\tau}(a)$ denota o filtro das vizinhanças de $a$ em $\langle X, \tau\rangle$ (cf. Comentário 1.1.2). Usando o Comentário 2.1.2 (vii) (2) (1), mostra-se facilmente que:

(vi) São equivalentes:

(1) $x_{i} \underset{i \in I}{\stackrel{\tau}{\longrightarrow}} \mathcal{F} a$.

(2) Existe uma base $\mathcal{B}$ para $\tau$ tal que, para todo $B \in \mathcal{N}_{\tau}(a) \cap \mathcal{B},\left\langle x_{i}\right\rangle_{i \in I}$ está $\mathcal{F}$ eventualmente em $B$.

(3) Para toda base $\mathcal{B}$ para $\tau$ e todo $B \in \mathcal{N}_{\tau}(a) \cap \mathcal{B},\left\langle x_{i}\right\rangle_{i \in I}$ está $\mathcal{F}$-eventualmente em $B$.

Com o uso dos Comentários 1.2.5 (vi) (1) e 2.1.2 (vii) (2) (1), conclui-se facilmente o corolário a seguir, que estabelece caracterizações de convergência de famílias de pontos em espaços hemimétricos com respeito a filtros.

Corolário 2.1.6. Dados um conjunto não vazio $I$, um filtro $\mathcal{F}$ sobre $I$, um espaço pré-métrico $\langle X, d\rangle$ tal que $X$ é não vazio, uma família $\left\langle x_{i}\right\rangle_{i \in I}$ de pontos de $X$ e um a $\in X$, considere as seguintes asserções: 
(a) A família $\left\langle d\left(x_{i}, a\right)\right\rangle_{i \in I} \mathcal{F}$-converge para 0 com respeito a $\tau_{2}$.

(b) Para todo $\varepsilon \in \mathbb{R}^{+},\left\langle x_{i}\right\rangle_{i \in I}$ está $\mathcal{F}$-eventualmente em $B_{d}(a, \varepsilon)$.

(c) A família $\left\langle x_{i}\right\rangle_{i \in I} \mathcal{F}$-converge para a com respeito a $\tau_{d}$.

Então:

(i) (a) é equivalente a (b).

(ii) (b) implica (c).

Suponha que d seja uma hemimétrica sobre $X$ ou equivalente a $d_{1, X}$. Então:

(iii) (b) é equivalente a (c).

Note que não vale em geral a recíproca da implicação no item ( $i$ i) do corolário acima. De fato: denote por $c_{1}$ a sequência em 3 que é constante de valor 1 e lembre-se que Cofin $(\omega)$ denota o filtro de Fréchet sobre $\omega$ (cf. Comentário 1.1.2). Considerando a prémétrica $d_{\min }$ sobre 3 (cf. Comentário 1.2.2), tem-se que $\tau_{d_{\min }}=\{\emptyset, 3\}$ (em virtude dos itens (iii) (1) e (ix) (1) do Comentário 1.2.5). Logo, para todo $k<3, c_{1}$ Cofin $(\omega)$-converge para $k$ com respeito a $\tau_{d_{\text {min }}}$. Em particular, $c_{1} \operatorname{Cofin}(\omega)$-converge para 2 com respeito a $\tau_{d_{\text {min }}}$. Contudo, não é verdade que $c_{1}$ está Cofin $(\omega)$-eventualmente em $B_{d_{\text {min }}}(2,1)$, pois $B_{d_{\min }}(2,1)=\{0,2\}$ (cf. Comentário 1.2.5(vii)(2) (3)), o que implica que

$$
\left\{n<\omega: c_{1}(n) \in B_{d_{\min }}(2,1)\right\}=\emptyset \notin \operatorname{Cofin}(\omega)
$$

No que segue, é garantido que a definição de convergência de famílias de pontos com respeito a filtros generaliza as definições usuais de convergência de redes e de filtros.

Comentário 2.1.7. Dado um conjunto dirigido $\langle\Delta, \leqslant\rangle$, considere a seguinte família:

$$
\mathcal{I}_{\leqslant}:=\{[\delta, \rightarrow[\leqslant: \delta \in \Delta\}
$$

Verifica-se corriqueiramente que:

(i) $\mathcal{I}_{\leqslant}$tem a PIF.

Suponha que $\Delta$ seja não vazio. Então, pode-se considerar o filtro gerado $\left(\left(\mathcal{I}_{\leqslant}\right)^{\bigcap}\right)^{\subseteq}$ (v. Comentário 1.1.3). Denote por $\mathcal{F}_{\leqslant}$o filtro $\left(\left(\mathcal{I}_{\leqslant}\right)^{\bigcap}\right)^{\subseteq}$. Dados um conjunto não vazio $X$, uma rede $\left\langle x_{\delta}\right\rangle_{\delta \in \Delta}$ em $X$, um $A \subseteq X$ e um $a \in X$, é corriqueiro mostrar que: 
(ii) $\left\langle x_{\delta}\right\rangle_{\delta \in \Delta}$ estar eventualmente em $A$ é condição necessária e suficiente para $\left\langle x_{\delta}\right\rangle_{\delta \in \Delta}$ estar $\mathcal{F}_{\leqslant}$-eventualmente em $A$.

Usando o item (ii), tem-se que:

(iii) $\left\langle x_{\delta}\right\rangle_{\delta \in \Delta}$ ser eventualmente constante de valor $a$ é condição necessária e suficiente para $\left\langle x_{\delta}\right\rangle_{\delta \in \Delta} \operatorname{ser} \mathcal{F}_{\leqslant}$-eventualmente constante de valor $a$.

(iv) $\left\langle x_{\delta}\right\rangle_{\delta \in \Delta}$ estar frequentemente em $A$ é condição necessária e suficiente para $\left\langle x_{\delta}\right\rangle_{\delta \in \Delta}$ estar $\mathcal{F}_{\leqslant}$-frequentemente em $A$.

Usando os itens (ii) e (iv), conclui-se que:

(v) $\left\langle x_{\delta}\right\rangle_{\delta \in \Delta}$ ser universal em $X$ é condição necessária e suficiente para $\left\langle x_{\delta}\right\rangle_{\delta \in \Delta}$ ser $\mathcal{F}_{\leqslant}$-universal em $X$.

Dados uma topologia $\tau$ sobre $X$ e um filtro $\mathcal{F}$ sobre $X$, é fácil verificar que:

(vi) $a$ é um limite de $\mathcal{F}$ com respeito a $\tau$ se, e somente se, $a$ for um $\mathcal{F}$-limite de id $X$ com respeito a $\tau$.

Usando o item (ii), conclui-se que:

(vii) $x_{\delta} \underset{\delta \in \Delta}{\stackrel{\tau}{\longrightarrow}} a$ se, e somente se, $x_{\delta} \underset{\delta \in \Delta}{\stackrel{\tau}{\longrightarrow}} \mathcal{F}_{\leqslant} a$.

Afirmamos que:

(viii) $\mathcal{F}_{\leqslant_{\omega}}=\operatorname{Cofin}(\omega)$.

De fato: fixe arbitrariamente um $B \subseteq \omega$. Por um lado, suponha que $B \in \mathcal{F}_{\leqslant_{\omega}}$. Fixe então um subconjunto não vazio e finito $A$ de $\omega$ tal que $\bigcap_{n \in A}[n, \omega[\subseteq B$. Considere o natural $m(A):=\max (A)$. Logo, $[m(A), \omega[\subseteq B$, o que equivale a $\omega \backslash B \subseteq m(A)$. Como $m(A)$ é um ordinal finito, segue que $\omega \backslash B$ é finito, o que equivale a $B \in \operatorname{Cofin}(\omega)$.

Por outro lado, suponha que $B \in \operatorname{Cofin}(\omega)$. Caso $B=\omega$, é claro que $B \in \mathcal{F}_{\leqslant \omega}$. Caso contrário, tem-se que o conjunto $\omega \backslash B$ é não vazio e finito. Logo, pode-se considerar o natural $m(B):=\max (\omega \backslash B)$. Tome um $n \in[m(B)+1, \omega[$ qualquer. Como $m(B)<n$, então $n \notin \omega \backslash B$, o que equivale a $n \in B$. Já que $n \in[m(B)+1, \omega$ [ foi tomado qualquer, segue que $\left[m(B)+1, \omega\left[\subseteq B\right.\right.$, o que implica que $B \in \mathcal{F}_{\leqslant \omega}$.

Com o uso dos itens (vii) e (viii) do Comentário 2.1.7, do Comentário 2.1.5 (i) e da Proposição 1.1.5, é fácil concluir o seguinte 
Corolário 2.1.8. Sejam $\langle\Delta, \leqslant\rangle$ um conjunto dirigido e $\langle X, \tau\rangle$ um espaço topológico tais que $\Delta$ $e X$ são não vazios e $a \in X$. Então:

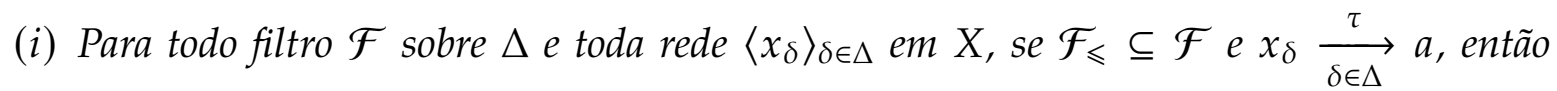
$x_{\delta} \underset{\delta \in \Delta}{\stackrel{\tau}{\longrightarrow}} \mathcal{F} a$.

(ii) Para todo ultrafiltro livre $\mathcal{U}$ sobre $\omega$ e toda sequência $\left\langle x_{n}\right\rangle_{n<\omega}$ em $X$, se $x_{n} \underset{n<\omega}{\stackrel{\tau}{\longrightarrow}}$ a, então $x_{n} \underset{n<\omega}{\stackrel{\tau}{\longrightarrow}} \mathcal{U} a$.

O exemplo a seguir garante que, em modelos de $\mathbf{Z F}+\mathbf{U T}$, é falsa a recíproca da implicação no item (ii) do corolário acima.

Exemplo 2.1.9. Sob UT, existem um ultrafiltro livre $\mathcal{U}$ sobre $\omega$, um espaço topológico $\langle X, \tau\rangle T_{0}$ tal que $X$ é não vazio e finito, uma sequência $s$ em $X$ e um $a \in X$ tais que $a$ é um $\mathcal{U}$-limite de $s$ que não é um limite de $s$ em $\langle X, \tau\rangle .^{2}$

Com efeito: denotando por $E$ e por $\chi_{E}$, respectivamente, o conjunto dos naturais pares e a função característica de $E$ definida em $\omega$, note que $\left\{n<\omega: \chi_{E}(n) \notin\{1\}\right\}=\omega \backslash E$. Denote por $S(2)$ o espaço topológico $\langle 2,\{\emptyset,\{1\}, 2\}\rangle$ (v. Comentário 1.2 .5 (viii) (1)). Já que o conjunto $\{1\}$ é um aberto em $S(2)$ e o conjunto $\omega \backslash E$ é infinito, tem-se que não é verdade que $\chi_{E}$ converge para 1 em $S(2)$. Como $\omega \backslash E \in \mathcal{P}(\omega) \backslash \operatorname{Cofin}(\omega)$, segue que a família Cofin $(\omega) \cup\{E\}$ tem a PIF (cf. Comentário 1.1.3(vi)(3)).

Assuma UT: fixe um ultrafiltro $\mathcal{U}$ sobre $\omega$ tal que $\operatorname{Cofin}(\omega) \cup\{E\} \subseteq \mathcal{U}$ (v. Teorema 1.1.6). Logo, $\mathcal{U}$ é livre (v. Proposição 1.1.5). É claro que:

$$
\begin{aligned}
\left\{n<\omega: \chi_{E}(n) \in\{1\}\right\} & =E \in \mathcal{U} \\
& \mathrm{e} \\
\left\{n<\omega: \chi_{E}(n) \in 2\right\} & =\omega \in \mathcal{U} .
\end{aligned}
$$

Já que os conjuntos $\{1\}$ e 2 são as únicas vizinhanças abertas de 1 em $S(2)$, conclui-se que $\chi_{E} \mathcal{U}$-converge para 1 em $S(2)$.

No que segue, são dadas caracterizações das definições de continuidade pontual,

\footnotetext{
${ }^{2}$ É interessante destacar que não é possível obter este exemplo em ZF, pois, no modelo $\mathcal{M} 27$ (i.e., o primeiro modelo de Pincus-Solovay), é verdadeira a asserção
}

$$
\text { para todo conjunto não vazio I e todo ultrafiltro } \mathcal{U} \text { sobre } I, \mathcal{U} \text { é um ultrafiltro principal, }
$$


de compacidade e de espaço topológico de Hausdorff por meio da convergência de famílias de pontos com respeito a filtros e ultrafiltros.

Teorema 2.1.10. Dados espaços topológicos $\langle X, \tau\rangle$ e $\left\langle X^{\prime}, \tau^{\prime}\right\rangle$ tais que $X$ e $X^{\prime}$ são não vazios, uma função $\gamma: X \rightarrow X^{\prime}$ e um $a \in X$, considere as seguintes asserções:

(a) $\gamma$ é contínua em a com respeito a $\tau$ e $\tau^{\prime}$.

(b) Dados um conjunto não vazio I, um filtro $\mathcal{F}$ sobre I e uma família $\left\langle x_{i}\right\rangle_{i \in I}$ de pontos de $X$, se $\left\langle x_{i}\right\rangle_{i \in I} \mathcal{F}$-convergir para a com respeito a $\tau$, então a família $\left\langle\gamma\left(x_{i}\right)\right\rangle_{i \in I} \mathcal{F}$-converge para $\gamma(a)$ com respeito a $\tau^{\prime}$.

Então:

(i) (a) implica (b).

(ii) Sob AC, (a) é equivalente a (b).

Suponha que $\langle X, \tau\rangle$ tenha caráter enumerável. Então:

(iii) Sob $\mathbf{A C}_{\omega}$, (a) é equivalente a (b).

Prova. $(i)$ : Fixe arbitrariamente um conjunto não vazio $I$, um filtro $\mathcal{F}$ sobre $I$, uma família $\left\langle x_{i}\right\rangle_{i \in I}$ de pontos de $X$ tal que $\left\langle x_{i}\right\rangle_{i \in I} \mathcal{F}$-converge para $a$ com respeito a $\tau$ e uma vizinhança $V^{\prime}$ de $\gamma(a)$ em $\left\langle X^{\prime}, \tau^{\prime}\right\rangle$. Suponha que $(a)$ valha. Então, o conjunto $\gamma^{-1}\left[V^{\prime}\right]$ é uma vizinhança de $a$ em $\langle X, \tau\rangle$. Então, $\left\langle x_{i}\right\rangle_{i \in I}$ está $\mathcal{F}$-eventualmente em $\gamma^{-1}\left[V^{\prime}\right]$. Já que $\gamma\left[\gamma^{-1}\left[V^{\prime}\right]\right] \subseteq V^{\prime}$, conclui-se que a família $\left\langle\gamma\left(x_{i}\right)\right\rangle_{i \in I}$ está $\mathcal{F}$-eventualmente em $V^{\prime}$ (cf. Comentário 2.1.2(vii)(1)(1)). Como se fixou arbitrariamente a vizinhança $V^{\prime}$ de $\gamma(a)$ em $\left\langle X^{\prime}, \tau^{\prime}\right\rangle$, segue que $\left\langle\gamma\left(x_{i}\right)\right\rangle_{i \in I} \mathcal{F}$-converge para $\gamma(a)$ com respeito a $\tau^{\prime}$. Portanto, já que se fixou arbitrariamente o conjunto não vazio $I$, o filtro $\mathcal{F}$ sobre $I$ e a família $\left\langle x_{i}\right\rangle_{i \in I}$ de pontos de $X$, conclui-se que $(b)$ vale.

(ii) e (iii) : Por $(i),(a)$ implica $(b)$. Por contraposição, provaremos que $(b)$ implica (a): suponha que (a) não valha. Fixe uma vizinhança $W$ de $\gamma(a)$ em $\left\langle X^{\prime}, \tau^{\prime}\right\rangle$ tal que, para todo $U \in \mathcal{N}_{\tau}(a)$, o conjunto $\gamma[U] \backslash W$ é não vazio, o que implica que o conjunto $A_{U}:=U \backslash \gamma^{-1}[W]$ é não vazio. Tome uma subfamília não vazia $\mathcal{A}$ qualquer de $\mathcal{N}_{\tau}(a)$. Considere então a família $\mathcal{A}_{W}:=\left\{A_{U}: U \in \mathcal{A}\right\}$.

Assuma $\mathbf{A C}$ ou, se $\mathcal{A}$ for enumerável, assuma $\mathbf{A C}_{\omega}$ : fixe uma função escolha $\phi$ para $\mathcal{A}_{W}$ (i.e., uma função de $\mathcal{A}_{W}$ em $\cup \mathcal{A}_{W}$ tal que, para todo $U \in \mathcal{A}$, 


$$
\left.\phi\left(A_{U}\right) \in A_{U}\right)
$$

Considere a relação binária $\leqslant$ sobre $\mathcal{A}$ que é definida pela seguinte sentença:

$$
\text { para quaisquer } U, V \in \mathcal{A}, V \leqslant U \text { se, e só se, } U \subseteq V \text {. }
$$

É fácil ver que $\langle\mathcal{A}, \leqslant\rangle$ é um conjunto dirigido. Notando que $\cup \mathcal{A}_{W} \subseteq X$, considere a rede $\left\langle x_{U}\right\rangle_{U \in \mathcal{A}}$ em $X$ que é definida pondo-se, para todo $U \in \mathcal{A}$,

$$
x_{U}:=\phi\left(A_{U}\right)
$$

Note que, para todo $U \in \mathcal{A}, \gamma\left(x_{U}\right) \notin W$. Logo, não é verdade que $\left\langle\gamma\left(x_{U}\right)\right\rangle_{U \in \mathcal{A}}$ converge para $\gamma(a)$ com respeito a $\tau^{\prime}$. Lembre-se que $\mathcal{F}_{\leqslant}$denota o filtro gerado $\left(\left(\mathcal{I}_{\leqslant}\right)^{\bigcap}\right)^{\subseteq}$ (cf. Comentário 2.1.7). Assim, tem-se que não é verdade que $\left\langle\gamma\left(x_{U}\right)\right\rangle_{U \in \mathcal{A}} \mathcal{F}_{\leqslant}$-converge para $\gamma(a)$ com respeito a $\tau^{\prime}$ (cf. Comentário 2.1.7(vii)). Suponha que se possa fixar uma base local $\mathcal{B}$ para $\tau$ em $a$ tal que $\mathcal{B} \subseteq \mathcal{A}$. Sendo assim, é fácil concluir que, para toda vizinhança $V$ de $a$ em $\langle X, \tau\rangle$, existe um $B \in \mathcal{B}$ tal que, para todo $U \in \mathcal{A}$, se $B \leqslant U$, então $x_{U} \in U \subseteq V$. Então, tem-se que $\left\langle x_{U}\right\rangle_{U \in \mathcal{A}}$ converge para $a$ com respeito a $\tau$. Logo, $\left\langle x_{U}\right\rangle_{U \in \mathcal{A}} \mathcal{F}_{\leqslant}$-converge para $a$ com respeito a $\tau$. Há dois casos a considerar:

- Caso $\langle X, \tau\rangle$ tenha caráter enumerável, fixe uma base local $\mathcal{B}$ para $\tau$ em a que é enumerável e tome $\mathcal{A}:=\mathcal{B}$.

- Caso contrário, tome $\mathcal{A}:=\mathcal{N}_{\tau}(a)$ e note que a família $\mathcal{N}_{\tau}(a) \cap \tau$ é uma base local para $\tau \operatorname{em} a$.

Portanto, conclui-se que $(b)$ não vale.

Teorema 2.1.11. Dado um espaço topológico $\langle X, \tau\rangle$ tal que Xé não vazio, considere as seguintes asserções:

(a) $\langle X, \tau\rangle$ é compacto.

(b) Dados um conjunto não vazio I, um ultrafiltro $\mathcal{U}$ sobre I e uma família $\left\langle x_{i}\right\rangle_{i \in I}$ de pontos de $X$, tem-se que $\left\langle x_{i}\right\rangle_{i \in I}$ é $\mathcal{U}$-convergente com respeito a $\tau$.

Então:

(i) (a) implica (b).

(ii) Sob UT, (a) é equivalente a (b). 
Prova. (i) : Por contraposição, provaremos que $(a)$ implica $(b)$ : suponha que $(b)$ não valha. Fixe um conjunto não vazio $I$, um ultrafiltro $\mathcal{U}$ sobre $I$ e uma família $\left\langle x_{i}\right\rangle_{i \in I}$ de pontos de $X$ tal que $\left\langle x_{i}\right\rangle_{i \in I}$ é $\mathcal{U}$-divergente com respeito a $\tau$. Considere a seguinte família:

$$
C:=\left\{V \in \tau:\left\langle x_{i}\right\rangle_{i \in I} \text { está } \mathcal{U} \text {-eventualmente em } X \backslash V\right\} .
$$

Mostraremos que $C$ é uma cobertura de $X$ : é claro que $\bigcup C \subseteq X$. Tome um $a \in X$ qualquer. Fixe uma vizinhança $W$ de $a$ em $\langle X, \tau\rangle$ tal que não é verdade que $\left\langle x_{i}\right\rangle_{i \in I}$ está $\mathcal{U}$-eventualmente em $W$, o que equivale a dizer que $\left\langle x_{i}\right\rangle_{i \in I}$ está $\mathcal{U}$-frequentemente em $X \backslash W$. Assim, tem-se que $\left\langle x_{i}\right\rangle_{i \in I}$ está $\mathcal{U}$-eventualmente em $X \backslash W$ (cf. Comentário 2.1.2(v) (2)). Fixe um $V \in \tau$ tal que $a \in V \subseteq W$. Já que $X \backslash W \subseteq X \backslash V$, tem-se que $\left\langle x_{i}\right\rangle_{i \in I}$ está $\mathcal{U}$-eventualmente em $X \backslash V$ (cf. Comentário 2.1.2(vii) (2) (1)). Logo, $V \in C$. Como se tomou $a \in X$ qualquer, então $X \subseteq \cup C$. Agora, para cada $A \subseteq X$, considere o conjunto $J_{A}:=\left\{i \in I: x_{i} \in A\right\}$. Note que $J_{X}=I$ e que, para todo $\mathcal{A} \subseteq \mathcal{P}(I), J \cup \mathcal{A}=\bigcup_{A \in \mathcal{A}} J_{A}$. Note também que, para toda vizinhança $V$ de $a$ em $\langle X, \tau\rangle, J_{V} \notin \mathcal{U}$, o que implica que $I \backslash J_{V} \in \mathcal{U}$. Suponha que se possa fixar uma subfamília finita $C^{\prime}$ de $C$ que é uma cobertura de $X$. Já que $X$ é não vazio, segue que $C^{\prime}$ é não vazia. Considere a família $\mathcal{J}_{C^{\prime}}:=\left\{I \backslash J_{V}: V \in C^{\prime}\right\}$. É claro que $\mathcal{J}_{C^{\prime}}$ é uma subfamília não vazia e finita de $\mathcal{U}$. Assim, tem-se que:

$$
\emptyset=I \backslash I=I \backslash J_{X}=I \backslash J \cup C^{\prime}=I \backslash \bigcup_{V \in C^{\prime}} J_{V}=\bigcap_{V \in C^{\prime}}\left(I \backslash J_{V}\right)=\cap \mathcal{J}_{C^{\prime}} \in \mathcal{U},
$$

uma contradição. Portanto, conclui-se que (a) não vale.

(ii) : Por $(i),(a)$ implica $(b)$. Por contraposição, provaremos que $(b)$ implica $(a)$ : suponha que (a) não valha. Fixe uma família não vazia $\mathcal{G}$ de fechados em $\langle X, \tau\rangle$ tal que $\mathcal{G}$ tem a PIF e $\cap \mathcal{G}=\emptyset$.

Assuma UT: fixe um ultrafiltro $\mathcal{U}$ sobre $X$ tal que $\mathcal{G} \subseteq \mathcal{U}$ (v. Teorema 1.1.6). Suponha que $\mathrm{id}_{X}$ seja $\mathcal{U}$-convergente com respeito a $\tau$. Fixe então um $a \in X$ tal que $\mathrm{id}_{X} \mathcal{U}$ converge para $a$ com respeito a $\tau$, o que equivale a dizer que $a$ é um limite de $\mathcal{U}$ com respeito a $\tau$ (cf. Comentário 2.1.7(vi)). Assim, tem-se que $\mathcal{N}_{\tau}(a) \subseteq \mathcal{U}$. Como $a \notin \bigcap \mathcal{G}$, fixe um $F \in \mathcal{G}$ tal que $a \notin F$. Então, tem-se que $F \in \mathcal{U}$ e que $a \in X \backslash F$. Já que $X \backslash F \in \tau$, segue que $X \backslash F \in \mathcal{N}_{\tau}(a)$, o que implica que $X \backslash F \in \mathcal{U}$. Logo,

$$
\emptyset=F \cap(X \backslash F) \in \mathcal{U}
$$

uma contradição. Portanto, conclui-se que (b) não vale. 
Teorema 2.1.12. Seja $\langle X, \tau\rangle$ um espaço topológico tal que Xé não vazio. Então, são equivalentes:

(i) $\langle X, \tau\rangle$ é de Hausdorff.

(ii) Dados um conjunto não vazio I, um filtro $\mathcal{F}$ sobre I e uma família $\left\langle x_{i}\right\rangle_{i \in I}$ de pontos de $X$, se $\left\langle x_{i}\right\rangle_{i \in I}$ for $\mathcal{F}$-convergente com respeito a $\tau$, então existe um único $\mathcal{F}$-limite de $\left\langle x_{i}\right\rangle_{i \in I}$ com respeito $a \tau$.

Prova. $(i) \Longrightarrow($ ii $)$ : Provaremos por redução ao absurdo: suponha que $(i)$ valha e que (ii) não valha. Fixe um conjunto não vazio $I$, um filtro $\mathcal{F}$ sobre $I$ e uma família $\left\langle x_{i}\right\rangle_{i \in I}$ de pontos de $X$ tal que $\left\langle x_{i}\right\rangle_{i \in I}$ é $\mathcal{F}$-convergente com respeito a $\tau$ e não é verdade que existe um único $\mathcal{F}$-limite de $\left\langle x_{i}\right\rangle_{i \in I}$ com respeito a $\tau$. Fixe então $a, b \in X$ tais que $a \neq b$ e $\left\langle x_{i}\right\rangle_{i \in I}$ $\mathcal{F}$-converge para $a$ e $\mathcal{F}$-converge para $b$ com respeito a $\tau$. Fixe agora uma vizinhança $V$ de $a$ e uma vizinhança $W$ de $b$ em $\langle X, \tau\rangle$ tais que $V \cap W=\emptyset$. Então, $\left\langle x_{i}\right\rangle_{i \in I}$ está $\mathcal{F}$-eventualmente em $V$ e está $\mathcal{F}$-eventualmente em $W$ e, por conseguinte, $\left\langle x_{i}\right\rangle_{i \in I}$ está $\mathcal{F}$-eventualmente em $\emptyset$ (cf. Comentário 2.1.2(viii) (1)). Logo,

$$
\emptyset=\left\{i \in I: x_{i} \in \emptyset\right\} \in \mathcal{F},
$$

uma contradição.

$(i i) \Longrightarrow(i)$ : Provaremos por contraposição: suponha que (i) não valha. Fixe $a, b \in X$ tais que $a \neq b$ e,

(*) para todo $U \in \mathcal{N}_{\tau}(a) \cap \tau$ e todo $V \in \mathcal{N}_{\tau}(b) \cap \tau$, o conjunto $U \cap V$ é não vazio.

Considere a família $\mathcal{N}:=\left(\mathcal{N}_{\tau}(a) \cup \mathcal{N}_{\tau}(b)\right) \cap \tau$. Note que $\mathcal{N}$ é não vazia (pois $X \in \mathcal{N}$ ). Tome uma subfamília não vazia e finita $\mathcal{A}$ qualquer de $\mathcal{N}$. Há dois casos a considerar:

- Caso $\mathcal{A} \in \mathcal{N}_{\tau}(a) \cap \tau$ ou $\mathcal{A} \in \mathcal{N}_{\tau}(b) \cap \tau$, é claro que $a \in \bigcap \mathcal{A}$ ou $b \in \cap \mathcal{A}$.

- Caso contrário, fixe $\mathcal{A}^{\prime} \subseteq \mathcal{N}_{\tau}(a) \cap \tau$ e $\mathcal{A}^{\prime \prime} \subseteq \mathcal{N}_{\tau}(b) \cap \tau$ tais que $\mathcal{A}=\mathcal{A}^{\prime} \cup \mathcal{A}^{\prime \prime}$. Já que $\mathcal{A}^{\prime}, \mathcal{A}^{\prime \prime} \subseteq \mathcal{A}$, tem-se que $\cap \mathcal{A}^{\prime} \in \mathcal{N}_{\tau}(a) \cap \tau$ e que $\cap \mathcal{A}^{\prime \prime} \in \mathcal{N}_{\tau}(b) \cap \tau$. Como $\cap \mathcal{A}=\left(\cap \mathcal{A}^{\prime}\right) \cap\left(\cap \mathcal{A}^{\prime \prime}\right)$, conclui-se usando $(*)$ que o conjunto $\cap \mathcal{A}$ é não vazio.

Já que a subfamília não vazia e finita $\mathcal{A}$ de $\mathcal{N}$ foi tomada qualquer, segue que $\mathcal{N}$ tem a PIF. Então, pode-se considerar o filtro $\mathcal{F}(\mathcal{N})$ sobre $X$ (v. Comentário 1.1.3). Como $\mathcal{N} \subseteq \mathcal{F}(\mathcal{N})$, é óbvio que $\mathcal{N}_{\tau}(a) \cap \tau \subseteq \mathcal{F}(\mathcal{N})$ e que $\mathcal{N}_{\tau}(a) \cap \tau \subseteq \mathcal{F}(\mathcal{N})$, o que implica que $\mathcal{N}_{\tau}(a) \subseteq \mathcal{F}(\mathcal{N})$ e que $\mathcal{N}_{\tau}(a) \subseteq \mathcal{F}(\mathcal{N})$. Logo, $a$ e $b$ são limites de $\mathcal{F}(\mathcal{N})$ com respeito a 
$\tau$, o que equivale a dizer que $\operatorname{id}_{X} \mathcal{F}(\mathcal{N})$-converge para $a$ e $\mathcal{F}(\mathcal{N})$-convergir para $b$ com respeito a $\tau$ (cf. Comentário 2.1.7 (vi)). Portanto, conclui-se que (ii) não vale.

Com o uso do Comentário 2.1.5 (i) e dos Teoremas 2.1.11 e 2.1.12, é fácil concluir o seguinte

Corolário 2.1.13. Dado um espaço topológico $\langle X, \tau\rangle$ tal que $X$ é não vazio, considere as seguintes asserções:

(a) $\langle X, \tau\rangle$ é compacto e de Hausdorff.

(b) Dados um conjunto não vazio I, um ultrafiltro $\mathcal{U}$ sobre I e uma família $\left\langle x_{i}\right\rangle_{i \in I}$ de pontos de $X$, tem-se que existe um único $\mathcal{U}$-limite de $\left\langle x_{i}\right\rangle_{i \in I}$ com respeito a $\tau$.

Então:

(i) (a) implica (b).

(ii) Sob UT, (a) é equivalente a (b).

Com o uso do Comentário 2.1.5 (ii) e dos Teoremas 2.1.11(i) e 2.1.12, é fácil concluir o seguinte

Corolário 2.1.14. Dados um conjunto não vazio $I$, um ultrafiltro $\mathcal{U}$ sobre $I$, um espaço topológico $\langle X, \tau\rangle$ de Hausdorff tal que $X$ é não vazio e uma família $\left\langle x_{i}\right\rangle_{i \in I}$ de pontos de $X$, se existir um $A \subseteq X$ tal que $\left\{x_{i}: i \in I\right\} \subseteq A e\left\langle A, \tau_{A}\right\rangle$ é compacto, então existe um único $\mathcal{U}$-limite de $\left\langle x_{i}\right\rangle_{i \in I}$ com respeito a $\tau$.

Note que não vale em geral a recíproca da implicação no corolário acima. De fato: denote por $D(\omega)$ o espaço topológico $\langle\omega, \mathcal{P}(\omega)\rangle$. É claro que $D(\omega)$ é de Hausdorff. Lembrando que $\mathcal{U}(0)$ denota o ultrafiltro principal sobre $\omega$ em 0 (cf. Comentário 1.1.2), tem-se que 0 é o único $\mathcal{U}(0)$-limite de $\mathrm{id}_{\omega}$ em $D(\omega)$ (cf. Comentário 2.1.5 (v) e Teorema 2.1.12). É claro que, para todo $A \subseteq \omega$, se $\left\{\operatorname{id}_{\omega}(n): n<\omega\right\} \subseteq A$, então $A=\omega$. Contudo, $D(\omega)$ não é compacto (cf. Proposição 1.1.17 (i) (5)).

O próximo corolário é imediato do Corolário 2.1.8 e do Teorema 2.1.12.

Corolário 2.1.15. Sejam $\langle\Delta, \leqslant\rangle$ um conjunto dirigido e $\langle X, \tau\rangle$ um espaço topológico de Hausdorff tais que $\Delta$ e $X$ são não vazios. Então: 
(i) Para todo filtro $\mathcal{F}$ sobre $\Delta$ e toda rede $\left\langle x_{\delta}\right\rangle_{\delta \in \Delta} e m X$, se $\mathcal{F} \leqslant \subseteq \mathcal{F} e\left\langle x_{\delta}\right\rangle_{\delta \in \Delta}$ for convergente com respeito a $\tau$, então $\mathcal{F}-\lim _{\delta \in \Delta}^{\tau} x_{\delta}=\lim _{\delta \in \Delta}^{\tau} x_{\delta}$.

(ii) Para todo ultrafiltro livre $\mathcal{U}$ sobre $\omega$ e toda sequência $\left\langle x_{n}\right\rangle_{n<\omega}$ em $X$, se $\left\langle x_{n}\right\rangle_{n<\omega}$ for convergente com respeito a $\tau$, então $\mathcal{U}-\lim _{n<\omega}^{\tau} x_{n}=\underset{n<\omega}{\tau} x_{n}$.

Note que, sob UT, a implicação no Corolário 2.1.14 e o item (ii) do Corolário 2.1.15 não valem em geral para espaços topológicos que não são de Hausdorff, mesmo que tais espaços sejam compactos. De fato: denote por $E$, por $\chi_{E}$ e por $S(2)$, respectivamente, o conjunto dos naturais pares, a função característica de $E$ definida em $\omega$ e o espaço topológico $\langle 2,\{\emptyset,\{1\}, 2\}\rangle$. No Exemplo 2.1.9, viu-se que, sob UT, existe um ultrafiltro livre $\mathcal{U}$ sobre $\omega$ tal que $\chi_{E} \mathcal{U}$-converge para 1 em $S(2)$. Como a única vizinhança aberta de 0 em $S(2)$ é o natural 2, então $\chi_{E} \mathcal{U}$-converge para 0 em $S(2)$. Além disso, tem-se que $S(2)$ é compacto (por ser finito) e não é de Hausdorff (pois qualquer vizinhança dos naturais 0 e 1 em $S(2)$ tem interseção não vazia).

O teorema a seguir é uma generalização nossa do resultado clássico que afirma que, em um produto cartesiano, a composição da projeção em uma coordenada com uma rede universal também é uma rede universal nessa coordenada.

Teorema 2.1.16. Dados um conjunto não vazio $I$, um filtro $\mathcal{F}$ sobre $I$, um conjunto não vazio $L$, uma família $\left\{X_{l}: l \in L\right\}$ de conjuntos tal que o produto cartesiano $\prod_{l \in L} X_{l}$ é não vazio e uma família $\left\langle\xi_{i}\right\rangle_{i \in I}$ de pontos de $\prod_{l \in L} X_{l}$, se $\left\langle\xi_{i}\right\rangle_{i \in I}$ for $\mathcal{F}$-universal em $\prod_{l \in L} X_{l}$, então, para todo $l \in L$, a família $\left\langle\xi_{i}(l)\right\rangle_{i \in I}$ é $\mathcal{F}$-universal em $X_{l}$.

Prova. Fixe arbitrariamente um $m \in L$. Tome um $A \subseteq X_{m}$ qualquer. Para cada $l \in L$, considere o seguinte conjunto:

$$
A_{l}:=\left\{\begin{aligned}
X_{l} \backslash A, & \text { se } l=m \\
X_{l}, & \text { caso contrário. }
\end{aligned}\right.
$$

Denote por $p_{m}$ a projeção $p_{L, X_{l}, m}$ (v. Comentário 1.1.23). É claro que:

$$
p_{m}\left[\prod_{l \in L} A_{l}\right] \subseteq A_{m}=X_{m} \backslash A .
$$

Além disso, tem-se que:

$$
p_{m}\left[\left(\prod_{l \in L} X_{l}\right) \backslash\left(\prod_{l \in L} A_{l}\right)\right] \subseteq A .
$$


Mostraremos que (**) vale: tome um $z \in p_{m}\left[\left(\prod_{l \in L} X_{l}\right) \backslash\left(\prod_{l \in L} A_{l}\right)\right]$ qualquer. Fixe um $\zeta \in\left(\prod_{l \in L} X_{l}\right) \backslash\left(\prod_{l \in L} A_{l}\right)$ tal que $z=\zeta(m)$. Fixe agora um $n \in L$ tal que $\zeta(n) \notin A_{n}$. Segue de $n \neq m$ que $A_{n}=X_{n}$, uma contradição. Então, $n=m$. Logo, $z \notin A_{m}=X_{m} \backslash A, \mathrm{o}$ que equivale a $z \in A$. Já que $z \in p_{m}\left[\left(\prod_{l \in L} X_{l}\right) \backslash\left(\prod_{l \in L} A_{l}\right)\right]$ foi tomado qualquer, segue que (**) vale. Agora, suponha que a família $\left\langle\xi_{i}(m)\right\rangle_{i \in I}$ esteja $\mathcal{F}$-frequentemente em $A$. Em virtude do Comentário 2.1.2(vii) (1)(2), conclui-se usando (*) que a família $\left\langle\xi_{i}\right\rangle_{i \in I}$ está $\mathcal{F}$-frequentemente em $\left(\prod_{l \in L} X_{l}\right) \backslash\left(\prod_{l \in L} A_{l}\right)$. Suponha que $\left\langle\xi_{i}\right\rangle_{i \in I}$ seja $\mathcal{F}$-universal em $\prod_{l \in L} X_{l}$. Assim, tem-se que $\left\langle\xi_{i}\right\rangle_{i \in I}$ está $\mathcal{F}$-eventualmente em $\left(\prod_{l \in L} X_{l}\right) \backslash\left(\prod_{l \in L} A_{l}\right)$. Em virtude do Comentário 2.1.2(vii) (1) (1), conclui-se usando $(* *)$ que $\left\langle\xi_{i}(m)\right\rangle_{i \in I}$ está $\mathcal{F}$ eventualmente em $A$. Como se tomou $A \subseteq X_{m}$ qualquer, então $\left\langle\xi_{i}(m)\right\rangle_{i \in I}$ é $\mathcal{F}$-universal em $X_{m}$. Portanto, já que $m \in L$ foi fixado arbitrariamente, conclui-se que, para todo $l \in L,\left\langle\xi_{i}(l)\right\rangle_{i \in I}$ é $\mathcal{F}$-universal em $X_{l}$.

O exemplo a seguir garante que não vale em geral a recíproca da implicação no Teorema 2.1.16.

Exemplo 2.1.17. Existem um conjunto não vazio e finito $X$ e uma sequência $\left\langle\xi_{n}\right\rangle_{n<\omega}$ em $X^{\omega}$ que é Cofin $(\omega)$-convergente em $\langle X, \mathcal{P}(X)\rangle^{\omega}$ tais que $\left\langle\xi_{n}\right\rangle_{n<\omega}$ não é Cofin $(\omega)$ universal em $X^{\omega}$ e, para todo $k<\omega$, a sequência $\left\langle\xi_{n}(k)\right\rangle_{n<\omega}$ é $\operatorname{Cofin}(\omega)$-universal em $\langle X, \mathcal{P}(X)\rangle$.

Com efeito: denote por $E$ e por $c_{1}$, respectivamente, o conjunto dos naturais pares e a sequência em 2 que é constante de valor 1 . Para cada $n<\omega$, denote por $\chi_{\{n\}}$ a função característica do conjunto $\{n\}$ definida em $\omega$ e considere a seguinte sequência em 2 :

$$
\widehat{n}:=\left\{\begin{aligned}
\left\langle 1-\chi_{\{n\}}(k)\right\rangle_{k<\omega}, & \text { se } n \in E ; \\
c_{1}, & \text { caso contrário. }
\end{aligned}\right.
$$

Note que a sequência $\langle\widehat{n}\rangle_{n<\omega}$ em $2^{\omega}$ é tal que, para quaisquer $n, k<\omega$,

$$
\widehat{n}(k)= \begin{cases}0, & \text { se } k=n \text { e } n \in E \\ 1, & \text { caso contrário. }\end{cases}
$$

Assim, tem-se que, para todo $k<\omega$, a sequência $\langle\widehat{n}(k)\rangle_{n<\omega}$ em 2 é eventualmente constante de valor 1 , o que implica que $\langle\widehat{n}(k)\rangle_{n<\omega}$ é Cofin $(\omega)$-universal em 2 (em virtude 
dos itens (iii) e (viii) do Comentário 2.1.7 e do Comentário 2.1.2 (iv) (1)). Considere o conjunto $A:=\left\{s \in 2^{\omega}: \exists m<\omega(s(m)=0)\right\}$. É fácil ver que, para todo $n<\omega, \widehat{n} \in A$ se, e somente se, $n \in E$. Segue disto que:

$$
\begin{aligned}
\{n<\omega: \widehat{n} \in A\} & =\{n<\omega: n \in E\}=E \notin \operatorname{Cofin}(\omega) \\
& \text { e } \\
\left\{n<\omega: \widehat{n} \in 2^{\omega} \backslash A\right\} & =\{n<\omega: \widehat{n} \notin A\}=\{n<\omega: n \notin E\}= \\
& =\omega \backslash E \notin \operatorname{Cofin}(\omega) .
\end{aligned}
$$

Então, conclui-se que $\langle\widehat{n}\rangle_{n<\omega}$ não é Cofin $(\omega)$-universal em $2^{\omega}$. Agora, denote por $D(2)$ o espaço topológico $\langle 2, \mathcal{P}(2)\rangle$. Mostraremos que $\langle\widehat{n}\rangle_{n<\omega} \operatorname{Cofin}(\omega)$-converge para $c_{1}$ na potência topológica $D(2)^{\omega}$ : fixe arbitrariamente uma vizinhança canônica $V$ de $c_{1}$ em $D(2)^{\omega}$. Fixe então uma família de conjuntos $\left\{V_{k}: k<\omega\right\}$ tal que, para todo $k<\omega, V_{k} \subseteq 2$ e para a qual

$$
V=\prod_{k<\omega} V_{k} \text { e o conjunto } M:=\left\{k<\omega: V_{k} \neq 2\right\} \text { é finito. }
$$

Tome um $m \in \omega \backslash M$ qualquer. É óbvio que $V_{m}=2$, o que implica que $\widehat{m}(m)=0 \in V_{m}$. Note que, para todo $k \in \omega \backslash\{m\}$,

$$
\widehat{m}(k)=1=c_{1}(k) \in V_{k} .
$$

Logo, $\widehat{m} \in V$. Como se tomou $m \in \omega \backslash M$ qualquer, então $\omega \backslash M \subseteq\{n<\omega: \widehat{n} \in V\}$. Já que $\omega \backslash M \in \operatorname{Cofin}(\omega)$, segue que $\langle\widehat{n}\rangle_{n<\omega}$ está Cofin $(\omega)$-eventualmente em $V$. Portanto, como se fixou arbitrariamente a vizinhança canônica $V$ de $c_{1}$ em $D(2)^{\omega}$, conclui-se usando o Comentário 2.1.5 (vi) que $\langle\widehat{n}\rangle_{n<\omega} \operatorname{Cofin}(\omega)$-converge para $c_{1}$ em $D(2)^{\omega}$.

O teorema a seguir é uma generalização de uma bem conhecida caracterização de convergência de redes em espaços produtos por meio da convergência de suas redes coordenadas.

Teorema 2.1.18. Dados um conjunto não vazio $I$, um filtro $\mathcal{F}$ sobre $I$, um conjunto não vazio $L$, uma família $\left\{\left\langle X_{l}, \tau_{l}\right\rangle: l \in L\right\}$ de espaços topológicos tal que o produto cartesiano $\prod_{l \in L} X_{l}$ é não vazio, uma família $\left\langle\xi_{i}\right\rangle_{i \in I}$ de pontos de $\prod_{l \in L} X_{l}$ e um $\alpha \in \prod_{l \in L} X_{l},\left\langle\xi_{i}\right\rangle_{i \in I} \mathcal{F}$-converge para $\alpha$ com respeito a $\bigotimes_{l \in L} \tau_{l}$ se, e somente se, para todo $l \in L$, a família $\left\langle\xi_{i}(l)\right\rangle_{i \in I} \mathcal{F}$-convergir para $\alpha(l)$ com respeito $a \tau_{l}$.

Prova. Por um lado, suponha que a família $\left\langle\xi_{i}\right\rangle_{i \in I} \mathcal{F}$-convirja para $\alpha$ com respeito a 
$\bigotimes_{l \in L} \tau_{l}$. Com o uso do Comentário 1.1.23 (ii) (2) e do Teorema 2.1.10 (i) conclui-se que, para todo $l \in L$, a família $\left\langle\xi_{i}(l)\right\rangle_{i \in I} \mathcal{F}$-converge para $\alpha(l)$ com respeito a $\tau_{l}$.

Por outro lado, fixe arbitrariamente uma vizinhança canônica $V$ de $\alpha$ em $\prod_{l \in L}\left\langle X_{l}, \tau_{l}\right\rangle$. Fixe então uma família de conjuntos $\left\{V_{l}: l \in L\right\}$ tal que, para todo $l \in L, V_{l} \in \tau_{l}$ e para a qual

$$
V=\prod_{l \in L} V_{l} \text { e o conjunto } K:=\left\{l \in L: V_{l} \neq X_{l}\right\} \text { é finito. }
$$

Para cada $l \in L$, considere o conjunto $J_{l}:=\left\{i \in I: \xi_{i}(l) \in V_{l}\right\}$. Suponha que, para cada $l \in L$, a família $\left\langle\xi_{i}(l)\right\rangle_{i \in I} \mathcal{F}$-convirja para $\alpha(l)$ com respeito a $\tau_{l}$. Logo, para todo $l \in L$, $J_{l} \in \mathcal{F}$. Considere o seguinte conjunto:

$$
J_{K}:=\left\{\begin{array}{cl}
I, & \text { se } K=\emptyset \\
\bigcap_{l \in K} J_{l}, & \text { caso contrário. }
\end{array}\right.
$$

Como $\mathcal{F}$ é um filtro sobre $I$ e $K$ é finito, tem-se que $J_{K} \in \mathcal{F}$. Tome um $j \in J_{K}$ e um $m \in L$ quaisquer. Há dois casos a considerar:

- Caso $m \in K$, tem-se que $j \in J_{m}$.

- Caso contrário, tem-se que $V_{m}=X_{m}$.

Logo, $\xi_{j}(m) \in V_{m}$. Já que $m \in L$ foi tomado qualquer, tem-se que $\xi_{j} \in V$. Como se tomou $j \in J_{K}$ qualquer, então $J_{K} \subseteq\left\{i \in I: \xi_{i} \in V\right\}$. Já que $J_{K} \in \mathcal{F}$, segue que $\left\langle\xi_{i}\right\rangle_{i \in I}$ está $\mathcal{F}$-eventualmente em $V$. Portanto, como se fixou arbitrariamente a vizinhança canônica $V$ de $\alpha$ em $\prod_{l \in L}\left\langle X_{l}, \tau_{l}\right\rangle$, conclui-se usando o Comentário 2.1.5 (vi) que $\left\langle\xi_{i}\right\rangle_{i \in I} \mathcal{F}$-converge para $\alpha$ com respeito a $\bigotimes_{l \in L} \tau_{l}$.

O corolário a seguir é imediato do Comentário 1.1.23 (iii) e do Teorema 2.1.18.

Corolário 2.1.19. Dados um conjunto não vazio $I$, um filtro $\mathcal{F}$ sobre $I$, espaços topológicos $\langle X, \tau\rangle$ e $\left\langle X^{\prime}, \tau^{\prime}\right\rangle$ tais que $X$ e $X^{\prime}$ são não vazios, uma família $\left\langle x_{i}\right\rangle_{i \in I}$ de pontos de $X$, uma família $\left\langle x_{i}^{\prime}\right\rangle_{i \in I}$ de pontos de $X^{\prime}$, um $a \in X$ e um $a^{\prime} \in X^{\prime}$, a família $\left\langle\left\langle x_{i}, x_{i}^{\prime}\right\rangle\right\rangle_{i \in I} \mathcal{F}$-converge para $\left\langle a, a^{\prime}\right\rangle$ com respeito a $\tau \otimes \tau^{\prime}$ se, e somente se, $\left\langle x_{i}\right\rangle_{i \in I} \mathcal{F}$-convergir para a com respeito a $\tau$ e $\left\langle x_{i}^{\prime}\right\rangle_{i \in I}$ $\mathcal{F}$-convergir para $a^{\prime}$ com respeito a $\tau^{\prime}$.

O próximo corolário é imediato do Corolário 2.1.19 e do Teorema 2.1.10 (i). 
Corolário 2.1.20. Sejam I um conjunto não vazio e $\mathcal{F}$ um filtro sobre I. Sejam $\langle X, \tau\rangle,\left\langle X^{\prime}, \tau^{\prime}\right\rangle$ $e\left\langle X^{\prime \prime}, \tau^{\prime \prime}\right\rangle$ espaços topológicos tais que $X, X^{\prime}$ e $X^{\prime \prime}$ são não vazios, $\left\langle x_{i}\right\rangle_{i \in I}$ uma família de pontos de $X,\left\langle x_{i}^{\prime}\right\rangle_{i \in I}$ uma família de pontos de $X^{\prime}, a \in X$ e $a^{\prime} \in X^{\prime}$ tais que $\left\langle x_{i}\right\rangle_{i \in I} \mathcal{F}$-converge para a com respeito a $\tau$ e $\left\langle x_{i}^{\prime}\right\rangle_{i \in I} \mathcal{F}$-converge para a com respeito a $\tau^{\prime}$. Seja $\gamma: X \times X^{\prime} \rightarrow X^{\prime \prime}$ uma função contínua em $\left\langle a, a^{\prime}\right\rangle$ com respeito $a \tau \otimes \tau^{\prime}$ e $\tau^{\prime \prime}$. Então, a família $\left\langle\gamma\left(x_{i}, x_{i}^{\prime}\right)\right\rangle_{i \in I} \mathcal{F}$-converge para $\gamma\left(a, a^{\prime}\right)$ com respeito a $\tau^{\prime \prime}$.

O corolário a seguir é imediato do Comentário 1.2.35 (iv) e do Corolário 2.1.20.

Corolário 2.1.21. Sejam I um conjunto não vazio e $\mathcal{F}$ um filtro sobre I. Sejam $\mathbb{K}$ um subcorpo de $\mathbb{C} e\left\langle\lambda_{i}\right\rangle_{i \in I} e\left\langle v_{i}\right\rangle_{i \in I}$ famílias de pontos de $\mathbb{K}$ que são $\mathcal{F}$-convergentes com respeito a $\tau_{\mathbb{K}}$. Então:

(i) (1) A família $\left\langle\lambda_{i}+v_{i}\right\rangle_{i \in I}$ é convergente com respeito a $\tau_{\mathbb{K}} e$

(2) $\mathcal{F}-\lim _{i \in I}^{\tau_{\mathrm{K}}}\left(\lambda_{i}+v_{i}\right)=\left(\mathcal{F}-\lim _{i \in I}^{\tau_{\mathrm{K}}} \lambda_{i}\right)+\left(\mathcal{F}-\lim _{i \in I}^{\tau_{\mathrm{K}}} v_{i}\right)$

(ii) (1) A família $\left\langle\lambda_{i} v_{i}\right\rangle_{i \in I}$ é convergente com respeito a $\tau_{\mathbb{K}} e$

(2) $\mathcal{F}-\lim _{i \in I}^{\tau_{\mathrm{K}}}\left(\lambda_{i} v_{i}\right)=\left(\mathcal{F}-\lim _{i \in I}^{\tau_{\mathrm{K}}} \lambda_{i}\right)\left(\mathcal{F}-\lim _{i \in I}^{\tau_{\mathrm{K}}} v_{i}\right)$.

Suponha que $\left\{\mathcal{F}-\lim _{i \in I}^{\tau_{\mathrm{K}}} \lambda_{i}\right\} \cup\left\{\lambda_{i}: i \in I\right\} \subseteq \mathbb{K} \backslash\{0\}$. Então:

(iii) (1) A família $\left\langle\left(\lambda_{i}\right)^{-1}\right\rangle_{i \in I}$ é convergente com respeito $a\left(\tau_{\mathbb{K}}\right)_{\mathbb{K} \backslash\{0\}} e$

(2) $\mathcal{F}-\lim _{i \in I}^{\tau_{\mathrm{K}}}\left(\lambda_{i}\right)^{-1}=\left(\mathcal{F}-\lim _{i \in I}^{\tau_{\mathrm{K}}} \lambda_{i}\right)^{-1}$.

O próximo corolário generaliza os resultados nos itens (i) e (ii) do Corolário 2.1.21 e é imediato do Corolário 2.1.20.

Corolário 2.1.22. Sejam I um conjunto não vazio e $\mathcal{F}$ um filtro sobre I. Sejam $\langle\mathbb{K}, \sigma\rangle$ um corpo topológico, $\left\langle\lambda_{i}\right\rangle_{i \in I}$ uma família de pontos de $\mathbb{K}$ e $\lambda \in \mathbb{K}$ tais que $\left\langle\lambda_{i}\right\rangle_{i \in I} \mathcal{F}$-converge para $\lambda$ com respeito a $\sigma$. Sejam $\langle X, \tau\rangle$ um espaço vetorial topológico sobre $\langle\mathbb{K}, \sigma\rangle,\left\langle x_{i}\right\rangle_{i \in I}$ e $\left\langle y_{i}\right\rangle_{i \in I}$ famílias de pontos de $X$ e $a, b \in X$ tais que $\left\langle x_{i}\right\rangle_{i \in I} \mathcal{F}$-converge para a com respeito a $\tau$ e $\left\langle y_{i}\right\rangle_{i \in I}$ $\mathcal{F}$-converge para $b$ com respeito a $\tau$. Então:

(i) A família $\left\langle x_{i}+y_{i}\right\rangle_{i \in I} \mathcal{F}$-converge para $a+b$ com respeito $a \tau$.

(ii) A família $\left\langle\lambda_{i} x_{i}\right\rangle_{i \in I} \mathcal{F}$-converge para $\lambda$ a com respeito a $\tau$. 
O corolário a seguir é imediato do Corolário 2.1.20, dos Comentários 1.2.11 (vii) e 1.1.15 (iii) (2) e do Teorema 2.1.12.

Corolário 2.1.23. Sejam I um conjunto não vazio e $\mathcal{F}$ um filtro sobre I. Sejam $\langle X, d\rangle$ um espaço pseudométrico tal que X é não vazio, $\left\langle x_{i}\right\rangle_{i \in I} e\left\langle y_{i}\right\rangle_{i \in I}$ famílias de pontos de X e a,b $b X$ tais que $\left\langle x_{i}\right\rangle_{i \in I} \mathcal{F}$-converge para a e $\left\langle y_{i}\right\rangle_{i \in I} \mathcal{F}$-converge para $b$ com respeito a $\tau_{d}$. Então:

(i) A família $\left\langle d\left(x_{i}, y_{i}\right)\right\rangle_{i \in I} \mathcal{F}$-converge para $d(a, b)$ com respeito a $\tau_{2}$.

(ii) Se d for uma métrica sobre $X e\left\langle d\left(x_{i}, y_{i}\right)\right\rangle_{i \in I} \mathcal{F}$-convergir para 0 com respeito a $\tau_{2}$, então $a=b$.

Note que o resultado no item (i) do corolário acima não vale em geral para espaços hemimétricos, mesmo que tais espaços sejam ultra-hemimétricos. Note também que o resultado no item ( $i$ i) desse corolário não vale em geral para espaços pseudométricos, mesmo que tais espaços sejam ultrapseudométricos. De fato: denotando por $c_{1}$ a sequência em 2 que é constante de valor 1 e considerando a ultra-hemimétrica $d_{\text {sec }}$ sobre 2 (cf. Comentário 1.2.2), tem-se que $c_{1} \operatorname{Cofin}(\omega)$-converge para 1 com respeito a $\tau_{d_{\mathrm{sec}}}$ (cf. Comentário 2.1.5(iv) (2)). Lembrando que $\tau_{d_{\mathrm{sec}}}=\{\emptyset,\{1\}, 2\}$ (cf. Comentário 1.2.5(viii)(1)), tem-se que a única vizinhança aberta de 0 em $\left\langle 2, \tau_{d_{\text {sec }}}\right\rangle$ é o natural 2. Como $\left\{n<\omega: c_{1}(n) \in 2\right\}=\omega$, segue que $c_{1} \operatorname{Cofin}(\omega)$-converge para 0 com respeito a $\tau_{d_{\mathrm{sec}}}$. Denotando por 0 a sequência nula em $\mathbb{R}$, é óbvio que $\left\langle d_{\mathrm{sec}}\left(c_{1}(n), c_{1}(n)\right)\right\rangle_{n<\omega}=0$ e, por conseguinte,

$$
\operatorname{Cofin}(\omega)-\lim _{n<\omega} d_{\mathrm{sec}}\left(c_{1}(n), c_{1}(n)\right)=0 \neq 1=d_{\mathrm{sec}}(0,1)
$$

Agora, considerando a ultrapseudométrica $d_{0,2}$ sobre 2 (cf. Comentário 1.2.2), é claro que:

$$
\left\langle d_{0,2}\left(c_{1}(n), c_{1}(n)\right)\right\rangle_{n<\omega}=\left(d_{0,2}\right)^{0} \circ c_{1}=\left(d_{0,2}\right)^{1} \circ c_{1}=0,
$$

o que implica que

$$
\begin{aligned}
\operatorname{Cofin}(\omega)-\lim _{n<\omega} d_{0,2}\left(c_{1}(n), c_{1}(n)\right) & =\operatorname{Cofin}(\omega)-\lim _{n<\omega} d_{0,2}\left(c_{1}(n), 0\right)= \\
& =\operatorname{Cofin}(\omega)-\lim _{n<\omega} d_{0,2}\left(c_{1}(n), 1\right)=0 .
\end{aligned}
$$

Logo, $c_{1}$ Cofin $(\omega)$-converge para 0 e $c_{1} \operatorname{Cofin}(\omega)$-converge para 1 com respeito a $\tau_{d_{0,2}}$ (em virtude dos itens (i) e (ii) do Corolário 2.1.6).

No que segue, é introduzida uma definição que generaliza a definição clássica de dominação entre funções reais. 
Comentário 2.1.24. Dados um conjunto não vazio $I$, uma subfamília não vazia $\mathcal{F}$ de $\mathcal{P}(I)$, subconjuntos não vazios $J$ e $J^{\prime}$ de $I$ tais $J \subseteq J^{\prime}$, um conjunto pré-ordenado $\langle X, \leqslant\rangle$ tal que $X$ é não vazio e funções $\xi: J \rightarrow X$ e $\xi^{\prime}: J^{\prime} \rightarrow X$, se $\left\{i \in J: \xi(i) \leqslant \xi^{\prime}(i)\right\} \in \mathcal{F}$, será dito que $\xi$ é $\mathcal{F}$-dominada por $\xi^{\prime}$. Sobre a potência cartesiana $X^{I}$, considere a relação binária $\leqslant_{\mathcal{F}}$ que é definida pela seguinte sentença:

para quaisquer funções $\xi, \zeta \in X^{I}, \xi \leqslant \mathcal{F} \zeta$ se, e só se, $\xi$ for $\mathcal{F}$-dominada por $\zeta$.

Suponha que $\mathcal{F}$ seja um filtro sobre $I$. Verifica-se facilmente que:

(i) $\leqslant \mathcal{F}$ é uma pré-ordem sobre $X^{I}$.

A pré-ordem $\leqslant \mathcal{F}$ será chamada de a relação de $\mathcal{F}$-dominação sobre $X^{I}$. É fácil ver que:

(ii) Para quaisquer funções $\xi, \zeta \in X^{I}$,

(1) $\xi \leqslant \zeta$ se, e somente se, $\xi \leqslant\{I\} \zeta$.

(2) se $\xi \leqslant \zeta$, então $\xi \leqslant \mathcal{F} \zeta$.

Para cada $n<\omega$, denote por $\chi_{\{n\}}$ a função característica do conjunto $\{n\}$ definida em $\omega$. Lembre-se que, para cada $n<\omega, \mathcal{U}(n)$ denota o ultrafiltro principal sobre $\omega$ em $n$ (cf. Comentário 1.1.2). É fácil ver que:

(iii) Para todo $k<\omega$,

(1) $\chi_{\{k\}} \leqslant \mathcal{U}(k+1) \chi_{\{k+1\}}$

(2) $\chi_{\{k\}} \nless \chi_{\{k+1\}}$.

Suponha que $\leqslant$ seja uma ordem parcial sobre $X$. Dado um $a \in X$, é fácil concluir que:

(iv) Para quaisquer funções $\xi, \zeta \in X^{I}$, se $\xi \leqslant \mathcal{F} \zeta$, então

(1) $\zeta$ estar $\mathcal{F}$-eventualmente em $] \leftarrow, a[\leqslant$ é condição suficiente para $\xi$ estar $\mathcal{F}$ eventualmente em $] \leftarrow, a[\leqslant \cdot$

(2) $\xi$ estar $\mathcal{F}$-eventualmente em $] a, \rightarrow[\leqslant$ é condição suficiente para $\zeta$ estar $\mathcal{F}$ eventualmente em $] a, \rightarrow[\leqslant \cdot$

Também é fácil concluir que:

(v) Para quaisquer funções $\xi, \zeta \in X^{I}, \xi \leqslant \mathcal{F} \zeta$ e $\zeta \leqslant_{\mathcal{F}} \xi$ se, e somente se, $\xi \equiv_{\mathcal{F}} \zeta$.

(vi) Para todo $k<\omega$, 
(1) (1) $\chi_{\{k\}} \equiv \mathcal{U}(k+2) \chi_{\{k+1\}}$.

(2) $\chi_{\{k\}} \neq \chi_{\{k+1\}}$.

(2) $\leqslant \mathcal{U}(k+2)$ não é uma ordem parcial sobre $\omega$.

Suponha que $\leqslant$ seja uma ordem total sobre $X$ e que $\mathcal{F}$ seja um ultrafiltro sobre $I$. Vê-se facilmente que:

(vii) $\leqslant \mathcal{F}$ é uma pré-ordem total sobre $X^{I}$.

Encerraremos esta seção com uma prova do teorema a seguir e uma nota sobre os resultados nesse teorema, que são generalizações nossas de resultados clássicos sobre convergência de redes em $\mathbb{R}$ com respeito a $\tau_{2}$.

Teorema 2.1.25. Sejam I um conjunto não vazio e $\mathcal{F}$ um filtro sobre I. Sejam $\langle X, \leqslant\rangle$ um conjunto totalmente ordenado tal que X é não vazio, $\left\langle x_{i}\right\rangle_{i \in I} e\left\langle y_{i}\right\rangle_{i \in I}$ famílias de pontos de $X$ que são $\mathcal{F}$-convergentes com respeito a $\tau_{\leqslant} e\left\langle z_{i}\right\rangle_{i \in I}$ uma família de pontos de $X$. Então:

(i) Se X for um denso segundo $\leqslant e\left\langle x_{i}\right\rangle_{i \in I} \leqslant \mathcal{F}\left\langle y_{i}\right\rangle_{i \in I}$, então $\mathcal{F}-\lim _{i \in I} x_{i} \leqslant \mathcal{F}-\lim _{i \in I} y_{i}$.

(ii) Se $\mathcal{F}-\lim _{i \in I}^{\tau_{\leqslant}} y_{i}=\mathcal{F}-\lim _{i \in I}^{\tau_{\leqslant}} x_{i},\left\langle x_{i}\right\rangle_{i \in I} \leqslant \mathcal{F}\left\langle z_{i}\right\rangle_{i \in I} e\left\langle z_{i}\right\rangle_{i \in I} \leqslant \mathcal{F}\left\langle y_{i}\right\rangle_{i \in I}$, então

(1) $\left\langle z_{i}\right\rangle_{i \in I}$ é $\mathcal{F}$-convergente com respeito $a \tau_{\leqslant} e$

(2) $\mathcal{F}-\lim _{i \in I}^{\tau_{\leqslant}} z_{i}=\mathcal{F}-\lim _{i \in I}^{\tau_{\leqslant}} x_{i}$

Prova. (i) : Provaremos por redução ao absurdo: suponha que não seja verdade que $\mathcal{F}-\lim _{i \in I}^{\tau_{\leqslant}} x_{i} \leqslant \mathcal{F}-\lim _{i \in I}^{\tau_{\leqslant}} y_{i}$. Sendo assim, $\mathcal{F}-\lim _{i \in I}^{\tau_{\leqslant}} y_{i}<\mathcal{F}-\lim _{i \in I}^{\tau_{\leqslant}} x_{i}$ (pois $\leqslant$ é uma pré-ordem total sobre $X)$. Suponha que $X$ seja um denso segundo $\leqslant$. Fixe um $a \in] \mathcal{F}-\lim _{i \in I} y_{i}, \mathcal{F}-\lim _{i \in I}^{\tau_{\leqslant}} x_{i}[\leqslant$ Então, $] \leftarrow, a\left[\leqslant\right.$ é uma vizinhança de $\mathcal{F}-\lim _{i \in I}^{\tau_{\leqslant}} y_{i}$ e $] a, \rightarrow\left[\leqslant\right.$ é uma vizinhança de $\mathcal{F}$ - $\lim _{i \in I}^{\tau_{\leqslant}} x_{i}$ em $\left\langle X, \tau_{\leqslant}\right\rangle$, o que implica que $\left\langle y_{i}\right\rangle_{i \in I}$ está $\mathcal{F}$-eventualmente em $] \leftarrow, a\left[\leqslant\right.$ e que $\left\langle x_{i}\right\rangle_{i \in I}$ está $\mathcal{F}$-eventualmente em $] a, \rightarrow\left[\leqslant \cdot\right.$ Como $\left\langle x_{i}\right\rangle_{i \in I} \leqslant \mathcal{F}\left\langle y_{i}\right\rangle_{i \in I}$, tem-se que $\left\langle x_{i}\right\rangle_{i \in I}$ está $\mathcal{F}$ eventualmente em $] \leftarrow, a[\leqslant($ cf. Comentário 2.1.24(iv) (1)). Além disso, tem-se que:

$$
] \leftarrow, a[\leqslant \cap] a, \rightarrow[\leqslant=\emptyset .
$$

(pois $\leqslant$ é uma ordem parcial sobre $X$ ). Então, $\left\langle x_{i}\right\rangle_{i \in I}$ está $\mathcal{F}$-eventualmente em $\emptyset$ (cf. Comentário 2.1.2(viii) (1)), o que equivale a

$$
\emptyset=\left\{i \in I: x_{i} \in \emptyset\right\} \in \mathcal{F},
$$


uma contradição.

(ii) (1) e (ii) (2) : Fixe arbitrariamente um $B \in \mathcal{N}_{\tau_{\leqslant}}\left(\mathcal{F}-\lim _{i \in I}^{\tau_{\leqslant}} x_{i}\right) \cap \mathcal{B}_{\leqslant}$. Assim, tem-se que $\left\langle x_{i}\right\rangle_{i \in I}$ está $\mathcal{F}$-eventualmente em $B$. Como $\mathcal{F}-\lim _{i \in I}^{\tau_{\leqslant}} y_{i}=\mathcal{F}-\lim _{i \in I}^{\tau_{\leqslant}} x_{i}$, note que $\left\langle y_{i}\right\rangle_{i \in I}$ está $\mathcal{F}$-eventualmente em $B$. Há quatro casos a considerar (v. Comentário 1.1.15):

- Caso $B=X$, é claro que $\left\langle z_{i}\right\rangle_{i \in I}$ está $\mathcal{F}$-eventualmente em $B$.

- Caso possamos fixar um $a \in X$ tal que $B=] \leftarrow, a\left[\leqslant\right.$, é claro que $\left\langle y_{i}\right\rangle_{i \in I}$ está $\mathcal{F}$ eventualmente em $] \leftarrow, a\left[\leqslant \cdot\right.$ Como $\left\langle z_{i}\right\rangle_{i \in I} \leqslant \mathcal{F}\left\langle y_{i}\right\rangle_{i \in I}$, tem-se então que $\left\langle z_{i}\right\rangle_{i \in I}$ está $\mathcal{F}$-eventualmente em $B$.

- Caso possamos fixar um $a \in X$ tal que $B=] a, \rightarrow\left[{ }_{\leqslant}\right.$é claro que $\left\langle x_{i}\right\rangle_{i \in I}$ está $\mathcal{F}$ eventualmente em $] a, \rightarrow\left[\leqslant \cdot\right.$ Como $\left\langle x_{i}\right\rangle_{i \in I} \leqslant \mathcal{F}\left\langle z_{i}\right\rangle_{i \in I}$, tem-se então que $\left\langle z_{i}\right\rangle_{i \in I}$ está $\mathcal{F}$-eventualmente em $B$ (cf. Comentário 2.1.24(iv) (2)).

- Caso possamos fixar um $a, b \in X$ tais que $B=] a, b\left[{ }_{\leqslant}\right.$é imediato concluir que $B=] \leftarrow, b[\leqslant \cap] a, \rightarrow\left[\leqslant \cdot\right.$ Logo, $\left\langle y_{i}\right\rangle_{i \in I}$ está $\mathcal{F}$-eventualmente em $] \leftarrow, b\left[\leqslant \mathrm{e}\left\langle x_{i}\right\rangle_{i \in I}\right.$ está $\mathcal{F}$-eventualmente em $] a, \rightarrow[\leqslant$. Com os mesmos argumentos dados nos dois casos anteriores, conclui-se que $\left\langle z_{i}\right\rangle_{i \in I}$ está $\mathcal{F}$-eventualmente em $] \leftarrow, b[\leqslant$ e que $\left\langle z_{i}\right\rangle_{i \in I}$ está $\mathcal{F}$-eventualmente em $] a, \rightarrow\left[\leqslant\right.$, o que equivale a dizer que $\left\langle z_{i}\right\rangle_{i \in I}$ está $\mathcal{F}$-eventualmente em $B$.

Portanto, como $B \in \mathcal{B}_{\leqslant} \cap \mathcal{N}_{\tau_{\leqslant}}\left(\mathcal{F}-\lim _{i \in I}^{\tau_{\leqslant}} x_{i}\right)$ foi fixado arbitrariamente, conclui-se usando o Comentário 2.1.5 (vi) que $\left\langle z_{i}\right\rangle_{i \in I} \mathcal{F}$-converge para $\mathcal{F}-\lim _{i \in I}^{\tau} x_{i}$ com respeito a $\tau_{\leqslant}$.

Note que não vale em geral a recíproca das implicações nos itens do Teorema 2.1.25. De fato: considere a sequência $s:=\left\langle(-1)^{n} 2^{-n}\right\rangle_{n<\omega}$ em $\mathbb{R}$ e denote por 0 a sequência nula em $\mathbb{R}$. Como $\lim _{n<\omega} s(n)=0$, então $s$ é Cofin $(\omega)$-convergente com respeito a $\tau_{2} \mathrm{e}$

$$
\operatorname{Cofin}(\omega)-\lim _{n<\omega} s(n)=\operatorname{Cofin}(\omega)-\lim _{n<\omega} 0(n)
$$

(em virtude dos itens (vii) e (viii) do Comentário 2.1.7 e do Comentário 2.1.5(iv)(2)). Denotando por $E$ o conjunto dos naturais pares, tem-se que:

$$
\{n<\omega: 0 \leqslant s(n)\}=E \notin \operatorname{Cofin}(\omega),
$$

o que equivale a $0 \nless{ }_{\operatorname{Cofin}}(\omega) s$. 


\subsection{Ultraprodutos de espaços pseudométricos e espaços pseudonormados}

Nesta seção, é apresentada uma generalização das definições de ultraproduto de espaços métricos e de ultraproduto de espaços normados e de Banach. Além disso, são estabelecidas generalizações de resultados demonstrados na literatura a que tivemos acesso ou (aparentemente) folclóricos.

Definição 2.2.1. Seja $I$ um conjunto não vazio. Sejam $\left\{\left\langle X_{i}, d_{i}\right\rangle: i \in I\right\}$ uma família de espaços pré-métricos tal que o produto cartesiano $\prod_{i \in I} X_{i}$ é não vazio, $A \subseteq \prod_{i \in I} X_{i} \mathrm{e}$ $\alpha, \beta \in \prod_{i \in I} X_{i}$. Seja $\langle X, d\rangle$ um espaço pré-métrico tal que $X$ é não vazio. Diremos que:

(i) $\alpha$ está finitamente distante de $\beta$ com respeito a $\left\{d_{i}: i \in I\right\}$ se valer a seguinte condição: existe um $\delta \in \mathbb{R}^{+}$tal que $\left\{d_{i}(\alpha(i), \beta(i)): i \in I\right\} \subseteq[0, \delta]$.

(ii) A é finitamente distal com respeito a $\left\{d_{i}: i \in I\right\}$ se valerem as seguintes condições:

(1) $A$ é não vazio.

(2) Para quaisquer $\xi, \xi^{\prime} \in A$, $\xi$ está finitamente distante de $\xi^{\prime}$ com respeito a $\left\{d_{i}: i \in I\right\}$.

Suponhamos que, para cada $i \in I,\left\langle X_{i}, d_{i}\right\rangle=\langle X, d\rangle$. Diremos que:

(iii) $\alpha$ está finitamente distante de $\beta$ segundo $d$ se $\alpha$ estiver finitamente distante de $\beta$ com respeito a $\left\{d_{i}: i \in I\right\}$.

(iv) $A$ é finitamente distal segundo $d$ se $A$ for finitamente distal com respeito a $\left\{d_{i}: i \in I\right\}$.

Comentário 2.2.2. Dados conjuntos não vazios $I$ e $X$, para cada $x \in X$, denotaremos por $c_{I, X, x}$ a função constante de valor $x$ de $I$ em $X$. Dado um subconjunto não vazio $A$ de $X^{I}$, se $\left\{c_{I, X, x}: x \in X\right\} \subseteq A$, será dito que $A$ contém qualquer constante em $X$. Dada uma pré-métrica $d$ sobre $X$, note que:

(i) A família $\left\{c_{I, X, x}: x \in X\right\}$ é finitamente distal segundo $d$. 
Dadas famílias $\left\{X_{i}: i \in I\right\}$ e $\left\{X_{i}^{\prime}: i \in I\right\}$ de conjuntos tais que os produtos cartesianos $\prod_{i \in I} X_{i}$ e $\prod_{i \in I} X_{i}^{\prime}$ são não vazios e uma família $\left\{\varphi_{i}: i \in I\right\}$ de funções tais que, para todo $i \in I, \varphi_{i}$ é uma função de $X_{i}$ em $X_{i}^{\prime}$, considere a função $\prod_{i \in I} \varphi_{i}: \prod_{i \in I} X_{i} \rightarrow \prod_{i \in I} X_{i}^{\prime}$ que é definida da seguinte maneira: para todo $\xi \in \prod_{i \in I} X_{i},\left(\prod_{i \in I} \varphi_{i}\right)(\xi)$ é a função de $I$ em $\bigcup_{i \in I} X_{i}^{\prime}$ que é definida pondo-se, para todo $j \in I$,

$$
\left(\left(\prod_{i \in I} \varphi_{i}\right)(\xi)\right)(j):=\varphi_{j}(\xi(j))
$$

A função $\prod_{i \in I} \varphi_{i}$ será chamada de a função produto de $\left\{\varphi_{i}: i \in I\right\}$. Dada uma família $\left\{d_{i}: i \in I\right\}$ de funções tais que, para todo $i \in I, d_{i}$ é uma pré-métrica sobre $X_{i}$, é imediato concluir que:

(ii) Para todo $B \subseteq \prod_{i \in I} X_{i}$, se $B$ for finitamente distal com respeito a $\left\{d_{i}: i \in I\right\}$, então, para todo $C \subseteq B$, se $C$ for não vazio, então $C$ é finitamente distal com respeito a $\left\{d_{i}: i \in I\right\}$.

Dada uma família $\left\{d_{i}^{\prime}: i \in I\right\}$ de funções tais que, para todo $i \in I$, $d_{i}^{\prime}$ é uma pré-métrica sobre $X_{i}^{\prime}$, suponha que, para cada $i \in I, \varphi_{i}$ seja não expansiva segundo $d_{i}$ e $d_{i}^{\prime}$. Sendo assim, é fácil verificar que:

(iii) Para todo $B \subseteq \prod_{i \in I} X_{i}$, se $B$ for finitamente distal com respeito a $\left\{d_{i}: i \in I\right\}$, então $\left(\prod_{i \in I} \varphi_{i}\right)[B]$ é finitamente distal com respeito a $\left\{d_{i}^{\prime}: i \in I\right\}$.

Agora, considere a relação binária $R_{I, d_{i}}^{<\infty}$ sobre $\prod_{i \in I} X_{i}$ que é definida pela seguinte sentença:

para quaisquer $\xi, \xi^{\prime} \in \prod_{i \in I} X_{i}, \xi R_{I, d_{i}}^{<\infty} \xi^{\prime}$ se, e só se, $\xi$ estiver finitamente distante de $\xi^{\prime}$ com respeito a $\left\{d_{i}: i \in I\right\}$.

Verifica-se facilmente que:

(iv) (1) $R_{I, d_{i}}^{<\infty}$ é reflexiva sobre $\prod_{i \in I} X_{i}$. 
(2) Se, para todo $i \in I, d_{i}$ for uma hemimétrica sobre $X_{i}$, então $R_{I, d_{i}}^{<\infty}$ é uma pré-ordem sobre $\prod_{i \in I} X_{i}$.

(3) Se, para todo $i \in I, d_{i}$ for uma pseudométrica sobre $X_{i}$, então $R_{I, d_{i}}^{<\infty}$ é uma relação de equivalência sobre $\prod_{i \in I} X_{i}$.

Afirmamos que:

(v) Sobre $\mathbb{R}$,

(1) existe uma pré-métrica $d$ satisfazendo a condição de não degeneração e de simetria que não é uma hemimétrica e tal que $R_{\omega, d}^{<\infty}$ não é uma pré-ordem sobre a potência cartesiana $\mathbb{R}^{\omega}$.

(2) existe uma ultra-hemimétrica $d$ que é uma quase métrica, que não é uma pseudométrica e tal que $R_{\omega, d}^{<\infty}$ não é uma relação de equivalência sobre a potência cartesiana $\mathbb{R}^{\omega}$.

De fato: denote por 0 a sequência nula em $\mathbb{R}$ e considere as sequências $s:=\langle n\rangle_{n<\omega} \mathrm{e}$ $t:=\langle-n\rangle_{n<\omega}$ em $\mathbb{R}$. Então:

(1) : Basta considerar a pré-métrica $d^{\wedge}$ sobre $\mathbb{R}$ (v. Comentário 1.2.2(vii)(1)) e notar que:

$$
\begin{aligned}
& \left\{d^{\wedge}(s(n), 0(n)): n<\omega\right\}=\left\{d^{\wedge}(0(n), t(n)): n<\omega\right\}=2 \subseteq[0,1] \\
& \text { e } \\
& \left\{d^{\wedge}(s(n), t(n)): n<\omega\right\}=\omega \backslash\{1\},
\end{aligned}
$$

o que implica que $s R_{\omega, d^{\wedge}}^{<\infty} 0$ e $0 R_{\omega, d^{\wedge}}^{<\infty} t$ e que não é verdade que $s R_{\omega, d^{\wedge}}^{<\infty} t$ (pois, para todo $\left.\varepsilon \in \mathbb{R}^{+}, \omega \backslash\{1\} \nsubseteq[0, \varepsilon]\right)$.

(2) : Basta considerar a ultra-hemimétrica $d^{\rightarrow}$ sobre $\mathbb{R}$ (v. Comentário 1.2.2(vii) (2)) e notar que:

$$
\begin{aligned}
& \left\{d^{\rightarrow}(0(n), s(n)): n<\omega\right\}=2 \subseteq[0,1] \\
& \text { e } \\
& \left\{d^{\rightarrow}(s(n), 0(n)): n<\omega\right\}=\omega \backslash\{1\},
\end{aligned}
$$

o que implica que $0 R_{\omega, d^{\rightarrow}}^{<\infty} s$ e que não é verdade que $s R_{\omega, d^{\rightarrow}}^{<\infty} 0$.

Agora, suponha que $d$ seja uma pseudométrica sobre $X$ e que, para cada $i \in I, d_{i}$ seja 
uma pseudométrica sobre $X_{i}$. Para cada $\xi \in \prod_{i \in I} X_{i}$, a classe de equivalência $[\xi]_{R_{I, d_{i}}^{<\infty}}$ será denotada por $\ell_{\infty, \xi}\left(I, X_{i}, d_{i}\right)$ (ou, simplesmente, por $\ell_{\infty, \xi}\left(I, d_{i}\right)$ ). Assim, tem-se que:

(vi) A família $\left\{\ell_{\infty, \zeta}\left(I, X_{i}, d_{i}\right): \zeta \in \prod_{i \in I} X_{i}\right\}$ é uma partição de $\prod_{i \in I} X_{i}$.

(vii) (1) Para todo $\xi \in \prod_{i \in I} X_{i}$

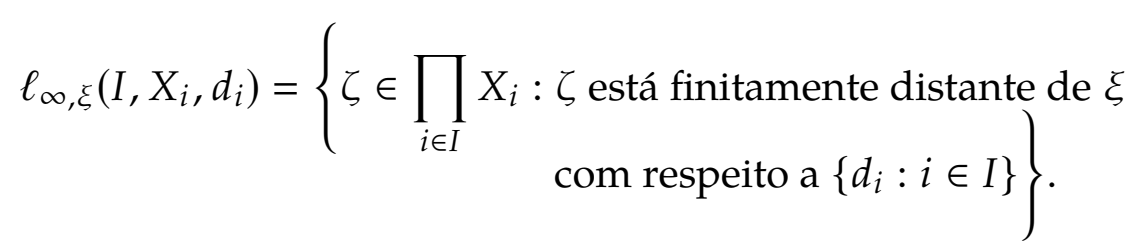

(2) Para quaisquer $\xi, \xi^{\prime} \in \prod_{i \in I} X_{i}, \ell_{\infty, \xi}\left(I, X_{i}, d_{i}\right)=\ell_{\infty, \xi^{\prime}}\left(I, X_{i}, d_{i}\right)$ se, e somente se, $\xi$ estiver finitamente distante de $\xi^{\prime}$ com respeito a $\left\{d_{i}: i \in I\right\}$.

(viii) (1) Para todo $\xi \in \prod_{i \in I} X_{i}$,

(1) $\ell_{\infty, \xi}\left(I, X_{i}, d_{i}\right)$ é finitamente distal com respeito a $\left\{d_{i}: i \in I\right\}$.

(2) Para todo $B \subseteq \prod_{i \in I} X_{i}$, se $\xi \in B$ e $B$ for finitamente distal com respeito a $\left\{d_{i}: i \in I\right\}$, então $B \subseteq \ell_{\infty, \xi}\left(I, X_{i}, d_{i}\right)$.

(2) $\prod_{i \in I} X_{i}$ é finitamente distal com respeito a $\left\{d_{i}: i \in I\right\}$ se, e somente se, para todo $\xi \in \prod_{i \in I} X_{i}, \ell_{\infty, \xi}\left(I, X_{i}, d_{i}\right)=\prod_{i \in I} X_{i}$

Suponha que, para cada $i \in I$, $d_{i}^{\prime}$ seja uma pseudométrica sobre $X_{i}^{\prime}$. Em virtude dos itens (iii) e (viii) (1) (2), tem-se que:

(ix) Para todo $\xi \in \prod_{i \in I} X_{i},\left(\prod_{i \in I} \varphi_{i}\right)\left[\ell_{\infty, \xi}\left(I, X_{i}, d_{i}\right)\right] \subseteq \ell_{\infty},\left(\prod_{i \in I} \varphi_{i}\right)(\xi)\left(I, X_{i}^{\prime}, d_{i}^{\prime}\right)$.

Denote por 0 a função identicamente nula de $I$ em $\mathbb{R}$. Com o uso da Proposição 1.2.15(v) e do Comentário 1.2.36 (ii) (2), é corriqueiro mostrar que:

(x) $\ell_{\infty, 0}\left(I, \mathbb{R}, d_{2}\right)=\ell_{\infty}(I)$. 
Comentário 2.2.3. Dados um conjunto não vazio $I$, uma família $\left\{\left\langle X_{i}, d_{i}\right\rangle: i \in I\right\}$ de espaços pré-métricos tal que o produto cartesiano $\prod_{i \in I} X_{i}$ é não vazio, um subconjunto $A$ de $\prod_{i \in I} X_{i}$ que é finitamente distal com respeito a $\left\{d_{i}: i \in I\right\}$ e $\alpha, \beta \in A$, fixe um $\delta \in \mathbb{R}^{+}$tal que $\left\{d_{i}(\alpha(i), \beta(i)): i \in I\right\} \subseteq[0, \delta]$. Sabe-se que $\left\langle[0, \delta],\left(\tau_{2}\right)_{[0, \delta]}\right\rangle$ é compacto (v. Teorema 1.2.17(ii)). Dado um ultrafiltro $\mathcal{U}$ sobre $I$, tem-se então que existe um único $\mathcal{U}$-limite da família $\left\langle d_{i}(\alpha(i), \beta(i))\right\rangle_{i \in I}$ com respeito a $\tau_{2}$ (cf. Corolário 2.1.14). Logo, podem ser consideradas as funções $\left(d_{i}\right)_{I, \infty, A},\left(d_{i}\right)_{I, \mathcal{U}, A}: A \times A \rightarrow \mathbb{R}$ que são definidas pondo-se, para quaisquer $\xi, \xi^{\prime} \in A$,

$$
\begin{aligned}
&\left(d_{i}\right)_{I, \infty, A}\left(\xi, \xi^{\prime}\right):=\sup \left\{d_{i}\left(\xi(i), \xi^{\prime}(i)\right): i \in I\right\} \\
& \mathrm{e} \\
&\left(d_{i}\right)_{I, \mathcal{U}, A}\left(\xi, \xi^{\prime}\right):=\mathcal{U}-\lim d_{i}\left(\xi(i), \xi^{\prime}(i)\right) .
\end{aligned}
$$

Com a utilização da monotonicidade da função supremo para uma família de funções com o mesmo domínio e limitadas superiormente no corpo ordenado $\mathbb{R}$ e com o uso dos Comentários 2.1.5 (iv) (2) e 2.1.24 (ii) (2) e do Teorema 2.1.25 (i), conclui-se que:

(i) (1) $\left(d_{i}\right)_{I, \infty, A}$ é uma pré-métrica sobre $A$.

(2) $\left(d_{i}\right)_{I, \mathcal{U}, A}$ é uma pré-métrica sobre $A$.

Para cada $j \in I$, denote por $p_{j}$ a projeção $p_{I, X_{i}, j}$ (v. Comentário 1.1.23). Lembre-se que, para cada $i \in I,\left(d_{i}\right)_{p_{i}[A]}$ denota a pré-métrica induzida por $d_{i}$ sobre $p_{i}[A]$ (cf. Comentário 1.2.6). Com a utilização da monotonicidade e da subaditividade da função supremo para uma família de funções com o mesmo domínio e limitadas superiormente no corpo ordenado $\mathbb{R}$ e com o uso do item (i), do Comentário 2.1.24 (ii) (2), do Teorema 2.1.25 (i) e do Corolário 2.1.21 (i), conclui-se que:

(ii) (1) Se, para todo $i \in I,\left(d_{i}\right)_{p_{i}[A]}$ for uma hemimétrica sobre $p_{i}[A]$, então

(1) $\left(d_{i}\right)_{I, \infty, A}$ é uma hemimétrica sobre $A$.

(2) $\left(d_{i}\right)_{I, \mathcal{U}, A}$ é uma hemimétrica sobre $A$.

(2) Se, para todo $i \in I,\left(d_{i}\right)_{p_{i}[A]}$ for uma pseudométrica sobre $p_{i}[A]$, então

(1) $\left(d_{i}\right)_{I, \infty, A}$ é uma pseudométrica sobre $A$.

(2) $\left(d_{i}\right)_{I, \mathcal{U}, A}$ é uma pseudométrica sobre $A$.

Verifica-se corriqueiramente que:

(iii) (1) Se, para todo $i \in I,\left(d_{i}\right)_{p_{i}[A]}$ for uma dimétrica sobre $p_{i}[A]$, então $\left(d_{i}\right)_{I, \infty, A}$ é uma dimétrica sobre $A$. 
(2) Se, para todo $i \in I,\left(d_{i}\right)_{p_{i}[A]}$ for uma quase métrica sobre $p_{i}[A]$, então $\left(d_{i}\right)_{I, \infty, A}$ é uma quase métrica sobre $A$.

Com a utilização da monotonicidade segundo a ordem lexográfica e da continuidade com respeito a $\tau_{2} \otimes \tau_{2}$ e $\tau_{2}$ da função máximo do corpo ordenado $\mathbb{R}$ e com o uso do item $(i)$, do Comentário 2.1.24 (ii) (2), do Teorema 2.1.25 (i) e do Corolário 2.1.20, conclui-se que:

(iv) (1) Se, para todo $i \in I,\left(d_{i}\right)_{p_{i}[A]}$ for uma ultra-hemimétrica sobre $p_{i}[A]$, então

(1) $\left(d_{i}\right)_{I, \infty, A}$ é uma ultra-hemimétrica sobre $A$.

(2) $\left(d_{i}\right)_{I, \mathcal{U}, A}$ é uma ultra-hemimétrica sobre $A$.

(2) Se, para todo $i \in I,\left(d_{i}\right)_{p_{i}[A]}$ for uma ultrapseudométrica sobre $p_{i}[A]$, então

(1) $\left(d_{i}\right)_{I, \infty, A}$ é uma ultrapseudométrica sobre $A$.

(2) $\left(d_{i}\right)_{I, \mathcal{U}, A}$ é uma ultrapseudométrica sobre $A$.

Com o uso dos itens (ii) (2) (1), (iii) (2) e (iv) (2) (1) e do Comentário 1.2.2 (i), conclui-se que:

(v) (1) Se, para todo $i \in I,\left(d_{i}\right)_{p_{i}[A]}$ for uma métrica sobre $p_{i}[A]$, então $\left(d_{i}\right)_{I, \infty, A}$ é uma métrica sobre $A$.

(2) Se, para todo $i \in I,\left(d_{i}\right)_{p_{i}[A]}$ for uma ultramétrica sobre $p_{i}[A]$, então $\left(d_{i}\right)_{I, \infty, A}$ é uma ultramétrica sobre $A$.

Suponha que, para cada $i \in I, d_{i}$ seja uma pseudométrica sobre $X_{i}$. Para cada $\xi \in \prod_{i \in I} X_{i}$, sempre que for conveniente, as pseudométricas $\left(d_{i}\right)_{I, \infty, \ell_{\infty, \xi}\left(I, X_{i}, d_{i}\right)}$ e $\left(d_{i}\right)_{I, \mathcal{U}, \ell_{\infty, \xi}\left(I, X_{i}, d_{i}\right)}$ serão denotadas, respectivamente, por $\left(d_{i}\right)_{I, \infty, \xi}$ e por $\left(d_{i}\right)_{I, \mathcal{U}, \xi}$. Usando o Comentário 2.2.2(vii) (2), tem-se que:

(vi) Para quaisquer $\xi, \xi^{\prime} \in \prod_{i \in I} X_{i}$, se $\ell_{\infty, \xi}\left(I, X_{i}, d_{i}\right)=\ell_{\infty, \xi^{\prime}}\left(I, X_{i}, d_{i}\right)$, então

(1) $\left(d_{i}\right)_{I, \infty, \xi}=\left(d_{i}\right)_{I, \infty, \xi^{\prime}}$

(2) $\left(d_{i}\right)_{I, \mathcal{U}, \xi}=\left(d_{i}\right)_{I, \mathcal{U}, \xi^{\prime}}$.

Dado um espaço pré-métrico $\langle X, d\rangle$ tal que $X$ é não vazio, caso tenhamos, para cada $i \in I,\left\langle X_{i}, d_{i}\right\rangle=\langle X, d\rangle$, denotaremos por $d_{\infty, A}$ e por $d_{\mathcal{U}, A}$, respectivamente, as pré-métricas $\left(d_{i}\right)_{I, \infty, A}$ e $\left(d_{i}\right)_{I, \mathcal{U}, A}$. Lembrando que $\ell_{\infty}=\ell_{\infty, 0}\left(\omega, \mathbb{R}, d_{2}\right)$ (v. Comentário 2.2.2 $(x)$ ), afirmamos que:

(vii) Para todo ultrafiltro livre $\mathcal{U}$ sobre $\omega$, a pseudométrica $\left(d_{2}\right) \mathcal{U}, 0$ sobre $\ell_{\infty}$ não é uma quase métrica sobre $\ell_{\infty}$. 
De fato: considerando a sequência $s:=\left\langle 2^{-n}\right\rangle_{n<\omega}$ em $\mathbb{R}$, tem-se que $s \in \ell_{\infty}$ e que $s \neq 0$. Suponha que se possa fixar um ultrafiltro livre $\mathcal{U}$ sobre $\omega$. Em virtude do Corolário 2.1.15(ii), tem-se que:

$$
\left(d_{2}\right) \mathcal{U}, 0(s, 0)=\mathcal{U}-\lim d_{2}(s(n), 0(n))=\mathcal{U}-\lim |s(n)|=\mathcal{U}-\lim s(n)=\lim s(n)
$$

Já que $\lim s(n)=0$, conclui-se que $\left(d_{2}\right) \mathcal{U}, 0(s, 0)=0$.

Comentário 2.2.4. Dados um conjunto não vazio $I$, uma família $\left\{\left\langle X_{i}, d_{i}\right\rangle: i \in I\right\}$ de espaços pseudométricos tal que o produto cartesiano $\prod_{i \in I} X_{i}$ é não vazio, lembre-se que, para cada $j \in I, p_{j}$ está denotando a projeção $p_{I, X_{i}, j}$ (v. Comentário 1.1.23). Dado um $A \subseteq \prod_{i \in I} X_{i}$, é claro que, para todo $i \in I,\left(d_{i}\right)_{p_{i}[A]}$ é uma pseudométrica sobre $p_{i}[A](\mathrm{v}$. Comentário 1.2.6). Suponha que $A$ seja finitamente distal com respeito a $\left\{d_{i}: i \in I\right\}$. Dado um ultrafiltro $\mathcal{U}$ sobre $I$, sabe-se que $\left(d_{i}\right)_{I, \mathcal{U}, A}$ é uma pseudométrica sobre $A$ (cf. Comentário 2.2.3 (ii) (2) (2)). Lembre-se que $R^{\left(d_{i}\right)_{I, \mathcal{U}, A}}$ denota a relação induzida por $\left(d_{i}\right)_{I, \mathcal{U}, A}$ sobre $A$ (cf. Comentário 1.2.12). Para cada $\xi \in A$, a classe de equivalência $[\xi]_{R^{\left(d_{i}\right), u, A}}$ será denotada por $c_{0, \mathcal{U}, \xi, A}\left(I, X_{i}, d_{i}\right)$ (ou, simplesmente, por $c_{0, \mathcal{U}, \xi, A}\left(I, d_{i}\right)$ ). Assim, tem-se que:

(i) A família $\left\{c_{0, \mathcal{U}, \zeta, A}\left(I, X_{i}, d_{i}\right): \zeta \in A\right\}$ é uma partição de $A$.

(ii) (1) Para todo $\xi \in A, c_{0, \mathcal{U}, \xi, A}\left(I, X_{i}, d_{i}\right)=\left\{\zeta \in A: \mathcal{U}-\lim d_{i}(\zeta(i), \xi(i))=0\right\}$.

(2) Para quaisquer $\xi, \xi^{\prime} \in A, c_{0, \mathcal{U}, \xi, A}\left(I, X_{i}, d_{i}\right)=c_{0, \mathcal{U}, \xi^{\prime}, A}\left(I, X_{i}, d_{i}\right)$ se, e somente se, $\mathcal{U}-\lim d_{i}\left(\xi(i), \xi^{\prime}(i)\right)=0$.

(iii) Para todo $\xi \in A, c_{0, \mathcal{U}, \xi, A}\left(I, X_{i}, d_{i}\right)=\left(\left(\left(d_{i}\right)_{I, \mathcal{U}, A}\right)^{\xi}\right)^{-1}[\{0\}]$.

Para cada $\xi \in \prod_{i \in I} X_{i}$, sempre que for conveniente, o conjunto $c_{0, \mathcal{U}, \xi, \ell_{\infty, \xi}\left(I, X_{i}, d_{i}\right)}\left(I, X_{i}, d_{i}\right)$ será denotado por $c_{0, \mathcal{U}, \xi}\left(I, X_{i}, d_{i}\right)$ (ou, simplesmente, por $c_{0, \mathcal{U}, \xi}\left(I, d_{i}\right)$ ). Lembre-se que, para cada $n<\omega, \mathcal{U}(n)$ denota o ultrafiltro principal sobre $\omega$ em $n$ (cf. Comentário 1.1.2). Afirmamos que:

(iv) Para todo $k<\omega$,

(1) $c_{0} \nsubseteq \mathcal{c}_{0, \mathcal{U}(k), 0}\left(\omega, \mathbb{R}, d_{2}\right)$.

(2) $c_{0, \mathcal{U}(k), 0}\left(\omega, \mathbb{R}, d_{2}\right) \nsubseteq c_{0}$. 
De fato: fixe arbitrariamente um $k<\omega$. Com o uso dos Comentários 2.1.5 (v) e 2.2.2 (x), conclui-se que:

$$
c_{0, \mathcal{U}(k), 0}\left(\omega, \mathbb{R}, d_{2}\right)=\left\{\zeta \in \ell_{\infty}: \zeta(k)=0\right\} .
$$

(1) : Considerando a sequência $s:=\left\langle 2^{-n}\right\rangle_{n<\omega}$ em $\mathbb{R}$, tem-se que $s \in c_{0}$. Como $0<s(k)$, tem-se também que $s \notin c_{0, \mathcal{U}(k), 0}\left(\omega, \mathbb{R}, d_{2}\right)$.

(2) : Denote por $\chi_{\{k\}}$ a função característica do conjunto $\{k\}$ definida em $\omega$ e considere a sequência $t:=\left\langle 1-\chi_{\{k\}}(n)\right\rangle_{n<\omega}$ em $\mathbb{R}$. Sendo assim, $t \in c_{0, \mathcal{U}(k), 0}\left(\omega, \mathbb{R}, d_{2}\right)$. Já que $t$ é eventualmente constante de valor 1 , tem-se que $t \notin c_{0}$ (em virtude da Proposição 1.1.18 (iii) e do Teorema 1.1.20(i)).

Agora, suponhamos que I seja infinito. Afirmamos que:

(v) Para todo ultrafiltro livre $\mathcal{U}$ sobre $I, c_{0}(I) \subseteq c_{0, \mathcal{U}, 0}\left(I, \mathbb{R}, d_{2}\right)$.

(vi) Sob UT, existe um ultrafiltro livre $\mathcal{U}$ sobre $\omega$ tal que $c_{0} \neq c_{0, \mathcal{U}, 0}\left(\omega, \mathbb{R}, d_{2}\right)$.

Com efeito:

(v) : Lembre-se que Cofin $(I)$ denota o filtro de Fréchet sobre I (cf. Comentário 1.1.2). Usando os itens (ii) (1) (1) e (v) (2) do Comentário 1.2.36, tem-se que:

$$
\begin{aligned}
c_{0}(I)=\left\{\zeta \in \ell_{\infty}(I):\right. & \forall \varepsilon \in \mathbb{R}^{+}\left(\langle|\zeta(i)|\rangle_{i \in I} \text { está Cofin }(I)\right. \text {-eventualmente } \\
& \text { em }[0, \varepsilon[)\}= \\
=\left\{\zeta \in \ell_{\infty}(I):\right. & \forall \varepsilon \in \mathbb{R}^{+}\left(\langle|\zeta(i)|\rangle_{i \in I} \text { está Cofin }(I)\right. \text {-eventualmente } \\
& \text { em }]-\varepsilon, \varepsilon[)\}= \\
=\left\{\zeta \in \ell_{\infty}(I):\right. & \forall \varepsilon \in \mathbb{R}^{+}\left(\left\langle d_{2}(\zeta(i), 0(i))\right\rangle_{i \in I}\right. \text { está Cofin(I)-eventualmente } \\
& \text { em } \left.\left.B_{d_{2}}(0, \varepsilon)\right)\right\} .
\end{aligned}
$$

Em virtude do Corolário 2.1.6 (i), tem-se então que:

$$
c_{0}(I)=\left\{\zeta \in \ell_{\infty}(I): \operatorname{Cofin}(I)-\lim d_{2}(\zeta(i), 0(i))=0\right\} .
$$

Suponha que se possa fixar um ultrafiltro livre $\mathcal{U}$ sobre $I$. Com o uso da Proposição 1.1.5 e dos Comentários 2.1.5 (i) e 2.2.2 (x), conclui-se que $c_{0}(I) \subseteq c_{0, \mathcal{U}, 0}\left(I, \mathbb{R}, d_{2}\right)$.

(vi) : Denotando por $E$ e por $\chi_{E}$, respectivamente, o conjunto dos naturais pares e a função característica de $E$ definida em $\omega$, considere a sequência $s:=\left\langle 1-\chi_{E}(n)\right\rangle_{n<\omega}$ em $\mathbb{R}$. Então, a subsequência $\langle s(2 k+1)\rangle_{k<\omega}$ de $s$ é constante de valor 1 , o que implica que $s \notin c_{0}($ v. Proposição 1.1.18(i)).

Assuma UT: com os mesmos argumentos dados no Exemplo 2.1.9 para usar UT, fixe um ultrafiltro livre $\mathcal{U}$ sobre $\omega$ tal que $E \in \mathcal{U}$. Note que, para toda vizinhança aberta $V$ 
de 0 em $\left\langle\mathbb{R}, \tau_{2}\right\rangle, E \subseteq\{n<\omega: s(n) \in V\}$, o que implica que s está $\mathcal{U}$-eventualmente em $V$. Logo,

$$
\left(d_{2}\right) \mathcal{U}, 0(s, 0)=\mathcal{U}-\lim d_{2}(s(n), 0(n))=\mathcal{U}-\lim |s(n)|=\mathcal{U}-\lim s(n)=0
$$

e, por conseguinte, $s \in c_{0, \mathcal{U}, 0}\left(\omega, \mathbb{R}, d_{2}\right)$.

A proposição a seguir é imediata dos Comentários 1.2.9 (v) (2) (2) e 2.2.4 (iii).

Proposição 2.2.5. Sejam I um conjunto não vazio e $\mathcal{U}$ um ultrafiltro sobre I. Sejam $\left\{\left\langle X_{i}, d_{i}\right\rangle: i \in I\right\}$ uma família de espaços pseudométricos tal que o produto cartesiano $\prod_{i \in I} X_{i} e ́$ não vazio e A um subconjunto de $\prod_{i \in I} X_{i}$ que é finitamente distal com respeito a $\left\{d_{i}: i \in I\right\}$. Então:

(i) Para todo $\xi \in A$,

(1) $c_{0, \mathcal{U}, \xi, A}\left(I, X_{i}, d_{i}\right)=\overline{\{\xi\}}^{\tau}\left(d_{i}\right)_{I}, U_{, A}$.

(2) $c_{0, \mathcal{U}, \xi, A}\left(I, X_{i}, d_{i}\right)$ é um fechado em $\left\langle A, \tau_{\left(d_{i}\right)_{I, \mathcal{U}, A}}\right\rangle$.

(ii) Para todo $\xi \in \prod_{i \in I} X_{i}$

(1) $c_{0, \mathcal{U}, \xi}\left(I, X_{i}, d_{i}\right)=\overline{\{\xi\}}^{\tau}\left(d_{i}\right)_{1, \mathcal{u}, \xi}$.

(2) $c_{0, \mathcal{U}, \xi}\left(I, X_{i}, d_{i}\right)$ é um fechado em $\left\langle\ell_{\infty, \xi}\left(I, X_{i}, d_{i}\right), \tau_{\left(d_{i}\right)_{I, \mathcal{u}, \xi}}\right\rangle$.

Teorema 2.2.6. Seja I um conjunto não vazio. Sejam $\left\{\left\langle X_{i}, d_{i}\right\rangle: i \in I\right\}$ uma família de espaços pseudométricos tal que o produto cartesiano $\prod_{i \in I} X_{i}$ é não vazio e A um subconjunto não vazio de $\prod_{i \in I} X_{i}$. Seja $\delta \in \mathbb{R}^{+}$. Então:

(i) $S e \bigcup_{i \in I} \operatorname{im}\left(\left(d_{i}\right)_{p_{i}[A]}\right) \subseteq[0, \delta]$, então

(1) A é finitamente distal com respeito $a\left\{d_{i}: i \in I\right\}$.

(2) (1) $\left(d_{i}\right)_{I, \mathcal{U}, A} / R^{\left(d_{i}\right)_{I, \mathcal{U}, A}}$ é limitada.

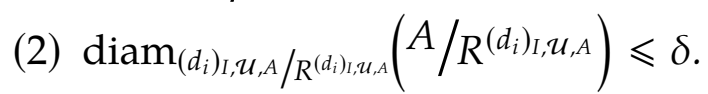

Suponha que A seja finitamente distal com respeito a $\left\{d_{i}: i \in I\right\}$ e que, para cada $i \in I,\left(d_{i}\right)_{p_{i}[A]}$ seja uma métrica sobre $p_{i}[A]$. Então: 
(ii) Se $\bigcup_{i \in I} \operatorname{im}\left(\left(d_{i}\right)_{p_{i}[A]}\right) \subseteq\{0\} \cup[\delta,+\infty[$, então

(1) (1) para todo $\xi \in A, c_{0, \mathcal{U}, \xi, A}\left(I, X_{i}, d_{i}\right)=[\xi]_{(\equiv \mathcal{U})_{A}}$.

(2) $R^{\left(d_{i}\right)_{I, \mathcal{U}, A}}=(\equiv \mathcal{U})_{A}$.

(2) (1) $\operatorname{im}\left(\left(d_{i}\right)_{I, \mathcal{U}, A} / R^{\left(d_{i}\right)_{I}, \mathcal{U}, A}\right) \subseteq\{0\} \cup[\delta,+\infty[$.

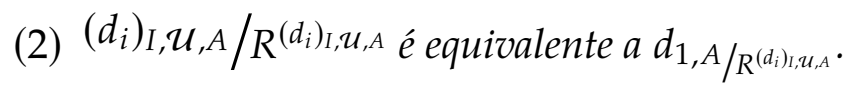

Prova. Inicialmente, lembre-se que, para cada $j \in I, p_{j}$ está denotando a projeção $p_{I, X_{i}, j}$ (v. Comentário 1.1.23).

(i) : Suponha que $\bigcup_{i \in I} \operatorname{im}\left(\left(d_{i}\right)_{p_{i}[A]}\right) \subseteq[0, \delta]$. Fixe arbitrariamente $\xi, \zeta \in A$.

(1) : Basta notar que:

$$
\left\{d_{i}(\xi(i), \zeta(i)): i \in I\right\} \subseteq \bigcup_{i \in I} \operatorname{im}\left(\left(d_{i}\right)_{p_{i}[A]}\right) .
$$

Como $\xi, \zeta \in A$ foram fixados arbitrariamente, segue que $A$ é finitamente distal com respeito a $\left\{d_{i}: i \in I\right\}$.

(2) (1) e (2) (2) : Lembre-se que $c_{I, \mathbb{R}, \delta}$ denota a função constante de valor $\delta$ de $I$ em $\mathbb{R}$ (cf. Comentário 2.2.2). Usando (*), conclui-se que $\left\langle d_{i}(\xi(i), \zeta(i))\right\rangle_{i \in I} \leqslant c_{I, \mathbb{R}, \delta}$, o que implica que $\left\langle d_{i}(\xi(i), \zeta(i))\right\rangle_{i \in I} \leqslant \mathcal{U} c_{I, \mathbb{R}, \delta}$ (cf. Comentário 2.1.24(ii) (2)). Logo,

$$
\begin{aligned}
\left(d_{i}\right)_{I, \mathcal{U}, A} / R^{\left(d_{i}\right)_{I, \mathcal{U}, A}}\left(c_{0, \mathcal{U}, \xi, A}\left(I, d_{i}\right), c_{0, \mathcal{U}, \zeta, A}\left(I, d_{i}\right)\right) & =\left(d_{i}\right)_{I, \mathcal{U}, A}(\xi, \zeta)= \\
& =\mathcal{U}-\lim d_{i}(\xi(i), \zeta(i)) \leqslant \\
& \leqslant \mathcal{U}-\lim c_{I, \mathbb{R}, \delta}=\delta
\end{aligned}
$$

(em virtude do Teorema 2.1.25 (i) e do Comentário 2.1.5(iv)(2)). Como $\xi, \zeta \in A$ foram fixados arbitrariamente, segue que $\left(d_{i}\right)_{I, \mathcal{U}, A} / R^{\left(d_{i}\right)_{I, \mathcal{U}, A}}$ é limitada e que

$$
\operatorname{diam}_{\left(d_{i}\right)_{I, \mathcal{U}, A} / R^{\left(d_{i}\right)_{1}, \mathcal{u}, A}}\left(A / R^{\left(d_{i}\right)_{I}, \mathcal{U}, A}\right) \leqslant \delta .
$$

(ii) : Suponha que $A$ seja finitamente distal com respeito a $\left\{d_{i}: i \in I\right\}$ e que, para cada $i \in I,\left(d_{i}\right)_{p_{i}[A]}$ seja uma métrica sobre $p_{i}[A]$. Suponha também que

$$
\bigcup_{i \in I} \operatorname{im}\left(\left(d_{i}\right)_{p_{i}[A]}\right) \subseteq\{0\} \cup[\delta,+\infty[.
$$


Fixe arbitrariamente $\xi, \zeta \in A$.

(1) (1) : Por um lado, suponha que $\zeta \in \mathcal{c}_{0, \mathcal{U}, \xi, A}\left(I, d_{i}\right)$. Sendo assim,

$$
\mathcal{U}-\lim d_{i}(\zeta(i), \xi(i))=0
$$

(cf. Comentário 2.2.4 (ii) (1)). Como $\left.B_{d_{2}}(0, \delta)=\right]-\delta, \delta[$ e, para todo $i \in I$,

$$
d_{i}(\zeta(i), \xi(i))=d_{2}\left(d_{i}(\zeta(i), \xi(i)), 0\right),
$$

segue que $\left\langle d_{i}(\zeta(i), \xi(i))\right\rangle_{i \in I}$ está $\mathcal{U}$-eventualmente em $]-\delta, \delta[$ (cf. Corolário 2.1.6(i)). Com o uso de $(*)$ e do Comentário 2.1.2 (ix) (1), conclui-se que:

$$
\left\langle d_{i}(\zeta(i), \xi(i))\right\rangle_{i \in I} \text { está } \mathcal{U} \text {-eventualmente em }\{0\} \cup[\delta,+\infty[\text {. }
$$

Como $]-\delta, \delta\left[\cap\left(\{0\} \cup\left[\delta,+\infty[)=\{0\}\right.\right.\right.$, segue que $\left\langle d_{i}(\zeta(i), \xi(i))\right\rangle_{i \in I}$ é $\mathcal{U}$-eventualmente constante de valor 0 (cf. Comentário 2.1.2 (viii) (1)). Notando que

$$
\left\{i \in I: d_{i}(\xi(i), \zeta(i)) \in\{0\}\right\}=\{i \in I: \zeta(i)=\xi(i)\},
$$

tem-se que $\zeta \equiv \mathcal{U} \xi$. Lembrando que $(\equiv \mathcal{U})_{A}$ denota a relação induzida por $\equiv \mathcal{U}$ sobre $A$ (cf. Comentário 1.1.8), tem-se então que $\zeta \in[\xi]_{(\equiv \mathcal{U})_{A}}$.

Por outro lado, suponha que $\zeta \in[\xi]_{(\equiv \mathcal{U})_{A}}$. Sendo assim, $\zeta \equiv \mathcal{U} \xi$. Usando $(\dagger)$, conclui-se que $\left\langle d_{i}(\zeta(i), \xi(i))\right\rangle_{i \in I}$ é $\mathcal{U}$-eventualmente constante de valor 0 . Segue disto que, para todo $\varepsilon \in \mathbb{R}^{+},\left\langle d_{i}(\zeta(i), \xi(i))\right\rangle_{i \in I}$ está $\mathcal{U}$-eventualmente em $]-\varepsilon, \varepsilon\left[=B_{d_{2}}(0, \varepsilon)\right.$. Logo,

$$
\mathcal{U}-\lim d_{i}(\zeta(i), \xi(i))=\mathcal{U}-\lim d_{2}\left(d_{i}(\zeta(i), \xi(i)), 0\right)=0,
$$

o que implica que $\zeta \in c_{0, \mathcal{U}, \xi, A}\left(I, d_{i}\right)$.

(1) (2) : É imediato de (1) (1).

(2) (1) : Suponha que $\left(d_{i}\right)_{I, \mathcal{U}, A} / R^{\left(d_{i}\right)_{I, \mathcal{U}, A}}\left(c_{0, \mathcal{U}, \xi, A}\left(I, d_{i}\right), c_{0, \mathcal{U}, \zeta, A}\left(I, d_{i}\right)\right) \neq 0$. Assim, tem-se

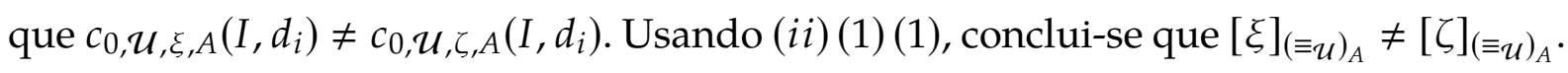
Logo, não é verdade que $\xi \equiv \mathcal{U} \zeta$. Usando $(\dagger)$, conclui-se que $\left\langle d_{i}(\xi(i), \zeta(i))\right\rangle_{i \in I}$ não é $\mathcal{U}$ eventualmente constante de valor 0 , o que equivale a dizer que $\left\langle d_{i}(\xi(i), \zeta(i))\right\rangle_{i \in I}$ está $\mathcal{U}$ frequentemente em $\mathbb{R} \backslash\{0\}$. Já que $\mathcal{U}$ é um ultrafiltro sobre $I$, segue que $\left\langle d_{i}(\xi(i), \zeta(i))\right\rangle_{i \in I}$ está $\mathcal{U}$-eventualmente em $\mathbb{R} \backslash\{0\}$ (cf. Comentário 2.1.2(v) (2)). Usando (**), conclui-se que $\left\langle d_{i}(\xi(i), \zeta(i))\right\rangle_{i \in I}$ está $\mathcal{U}$-eventualmente em $(\mathbb{R} \backslash\{0\}) \cap(\{0\} \cup[\delta,+\infty[)=[\delta,+\infty[$, o que equivale a $c_{I, \mathbb{R}, \delta} \leqslant \mathcal{U}\left\langle d_{i}(\xi(i), \zeta(i))\right\rangle_{i \in I}$. Então,

$$
\begin{aligned}
\delta=\mathcal{U}-\lim c_{I, \mathbb{R}, \delta} & \leqslant \mathcal{U}-\lim d_{i}(\xi(i), \zeta(i))=\left(d_{i}\right)_{I, \mathcal{U}, A}(\xi, \zeta)= \\
& =\left(d_{i}\right)_{I, \mathcal{U}, A} / R^{\left(d_{i}\right)_{I, \mathcal{U}, A}}\left(c_{0, \mathcal{U}, \xi, A}\left(I, d_{i}\right), c_{0, \mathcal{U}, \zeta, A}\left(I, d_{i}\right)\right) .
\end{aligned}
$$


Portanto, conclui-se que im $\left(\left(d_{i}\right)_{I, \mathcal{U}, A} / R^{\left(d_{i}\right)_{I, \mathcal{U}, A}}\right) \subseteq\{0\} \cup[\delta,+\infty[$.

(2) (2) : Segue imediatamente de (2) (1), do Comentário 1.2.12(x) (2) e do Comentário $1.2 .5(i v)(2)$.

Para estabelecermos os próximos resultados desta seção, iremos generalizar nossa definição de conjunto finitamente distal na seguinte

Definição 2.2.7. Sejam $I$ um conjunto não vazio e $\mathcal{U}$ um ultrafiltro sobre $I$. Sejam $\left\{\left\langle X_{i}, d_{i}\right\rangle: i \in I\right\}$ uma família de espaços pseudométricos tal que o produto cartesiano $\prod_{i \in I} X_{i}$ é não vazio e $A \subseteq \prod_{i \in I} X_{i}$. Seja $\langle X, d\rangle$ um espaço pseudométrico tal que $X$ é não vazio. Diremos que:

(i) $A$ é sup-finitamente distal com respeito a $\left\{d_{i}: i \in I\right\}$ se valerem as seguintes condições:

(1) A é finitamente distal com respeito a $\left\{d_{i}: i \in I\right\}$.

(2) Para todo $\zeta \in A$, existe uma função $\Lambda: \ell_{\infty, \zeta}\left(I, X_{i}, d_{i}\right) \rightarrow A$ tal que, para todo $\eta \in \ell_{\infty, \zeta}\left(I, X_{i}, d_{i}\right)$ e todo $\eta^{\prime} \in A$,

$$
\left(d_{i, \infty, A}\left(\Lambda(\eta), \eta^{\prime}\right) \leqslant\left(d_{i}\right)_{I, \infty, \zeta}\left(\eta, \eta^{\prime}\right) .\right.
$$

(ii) $A$ é $\mathcal{U}$-finitamente distal com respeito a $\left\{d_{i}: i \in I\right\}$ se valerem as seguintes condições:

(1) A é finitamente distal com respeito a $\left\{d_{i}: i \in I\right\}$.

(2) Para todo $\zeta \in A$, existe uma função $\Phi: \ell_{\infty, \zeta}\left(I, X_{i}, d_{i}\right) \rightarrow A$ tal que, para todo $\eta \in \ell_{\infty, \zeta}\left(I, X_{i}, d_{i}\right)$ e todo $\eta^{\prime} \in A$,

$$
\left(d_{i}\right)_{I, \mathcal{U}, A}\left(\Phi(\eta), \eta^{\prime}\right) \leqslant\left(d_{i}\right)_{I, \mathcal{U}, \zeta}\left(\eta, \eta^{\prime}\right)
$$

(iii) $A$ é $\mathcal{U}$-sup-finitamente distal com respeito a $\left\{d_{i}: i \in I\right\}$ se valerem as seguintes condições:

(1) A é finitamente distal com respeito a $\left\{d_{i}: i \in I\right\}$.

(2) Para todo $\zeta \in A$, existe uma função $\Psi: \ell_{\infty, \zeta}\left(I, X_{i}, d_{i}\right) \rightarrow A$ tal que, para todo $\eta \in \ell_{\infty, \zeta}\left(I, X_{i}, d_{i}\right)$ e todo $\eta^{\prime} \in A$,

$$
\begin{gathered}
\left(d_{i}\right)_{I, \infty, A}\left(\Psi(\eta), \eta^{\prime}\right) \leqslant\left(d_{i}\right)_{I, \infty, \zeta}\left(\eta, \eta^{\prime}\right) \\
\mathrm{e} \\
\left(d_{i}\right)_{I, \mathcal{U}, A}\left(\Psi(\eta), \eta^{\prime}\right) \leqslant\left(d_{i}\right)_{I, \mathcal{U}, \zeta}\left(\eta, \eta^{\prime}\right) .
\end{gathered}
$$


Suponhamos que, para cada $i \in I,\left\langle X_{i}, d_{i}\right\rangle=\langle X, d\rangle$. Diremos que:

(iv) $A$ é sup-finitamente distal segundo $d$ se $A$ for sup-finitamente distal com respeito a $\left\{d_{i}: i \in I\right\}$.

(v) $A$ é $\mathcal{U}$-finitamente distal segundo $d$ se $A$ for $\mathcal{U}$-finitamente distal com respeito a $\left\{d_{i}: i \in I\right\}$.

(vi) $A$ é $\mathcal{U}$-sup-finitamente distal segundo $d$ se $A$ for $\mathcal{U}$-sup-finitamente distal com respeito a $\left\{d_{i}: i \in I\right\}$.

Proposição 2.2.8. Sejam I um conjunto não vazio e $\mathcal{U}$ um ultrafiltro sobre I. Sejam $\left\{\left\langle X_{i}, d_{i}\right\rangle: i \in I\right\}$ uma família de espaços pseudométricos tal que o produto cartesiano $\prod_{i \in I} X_{i}$ é não vazio e $A \subseteq \prod_{i \in I} X_{i}$. Então:

(i) Se A for $\mathcal{U}$-sup-finitamente distal com respeito a $\left\{d_{i}: i \in I\right\}$, então

(1) A é sup-finitamente distal com respeito a $\left\{d_{i}: i \in I\right\}$.

(2) A é $\mathcal{U}$-finitamente distal com respeito a $\left\{d_{i}: i \in I\right\}$.

(ii) Para todo $\xi \in \prod_{i \in I} X_{i}$

(1) $\{\xi\}$ é $\mathcal{U}$-sup-finitamente distal com respeito $a\left\{d_{i}: i \in I\right\}$.

(2) $\ell_{\infty, \xi}\left(I, X_{i}, d_{i}\right)$ é $\mathcal{U}$-sup-finitamente distal com respeito a $\left\{d_{i}: i \in I\right\}$.

Prova. (i) e (ii) (1) : São óbvios.

(ii) (2) : Fixe arbitrariamente um $\xi \in \prod_{i \in I} X_{i}$. Sabe-se que $\ell_{\infty, \xi}\left(I, d_{i}\right)$ é finitamente distal com respeito a $\left\{d_{i}: i \in I\right\}$ (v. Comentário 2.2.2(viii)(1)(1)). Fixe arbitrariamente um $\zeta \in \ell_{\infty, \xi}\left(I, d_{i}\right)$. Lembre-se que $\ell_{\infty, \zeta}\left(I, d_{i}\right)=\ell_{\infty, \xi}\left(I, d_{i}\right)$ (v. Comentário 2.2.2(vii)), o que implica que $\left(d_{i}\right)_{I, \infty, \zeta}=\left(d_{i}\right)_{I, \infty, \xi}$ e que $\left(d_{i}\right)_{I, \mathcal{U}, \zeta}=\left(d_{i}\right)_{I, \mathcal{U}, \xi}$ (cf. Comentário 2.2.3(vi)). Logo, para todo $\eta \in \ell_{\infty, \zeta}\left(I, d_{i}\right)$ e todo $\eta^{\prime} \in \ell_{\infty, \xi}\left(I, d_{i}\right)$,

$$
\begin{aligned}
\left(d_{i}\right)_{I, \infty, \xi}\left(\operatorname{id}_{\ell_{\infty} \zeta\left(I, d_{i}\right)}(\eta), \eta^{\prime}\right) & =\left(d_{i}\right)_{I, \infty, \zeta}\left(\eta, \eta^{\prime}\right) \\
& \mathrm{e} \\
\left(d_{i}\right)_{I, \mathcal{U}, \xi}\left(\operatorname{id}_{\ell_{\infty, \zeta}\left(I, d_{i}\right)}(\eta), \eta^{\prime}\right) & =\left(d_{i}\right)_{I, \mathcal{U}, \zeta}\left(\eta, \eta^{\prime}\right) .
\end{aligned}
$$


Em vista dos resultados na Proposição 2.2.8, é natural que façamos as seguintes perguntas, para as quais ainda não temos uma resposta:

Questão 2.1. Existem um conjunto não vazio $I$, um ultrafiltro $\mathcal{U}$ sobre $I$, uma família $\left\{\left\langle X_{i}, d_{i}\right\rangle: i \in I\right\}$ de espaços pseudométricos tal que o produto cartesiano $\prod_{i \in I} X_{i}$ é não vazio e um $A \subseteq \prod_{i \in I} X_{i}$ tais que

(a) $A$ é sup-finitamente distal e $\mathcal{U}$-finitamente distal com respeito a $\left\{d_{i}: i \in I\right\}$ e não é $\mathcal{U}$-sup-finitamente distal com respeito a $\left\{d_{i}: i \in I\right\}$ ?

(b) $A$ é $\mathcal{U}$-sup-finitamente distal com respeito a $\left\{d_{i}: i \in I\right\}$ e $A$ não é unitário nem é verdade que existe um $\zeta \in \prod_{i \in I} X_{i}$ tal que $A=\ell_{\infty, \zeta}\left(I, X_{i}, d_{i}\right)$ ?

Os resultados no teorema a seguir são generalizações nossas de outros que estão demonstrados no contexto ingênuo ${ }^{3}$ dos espaços métricos completos.

Teorema 2.2.9. Seja I um conjunto não vazio. Sejam $\left\{\left\langle X_{i}, d_{i}\right\rangle: i \in I\right\}$ uma família de espaços pseudométricos completos tal que o produto cartesiano $\prod_{i \in I} X_{i}$ é não vazio e A um subconjunto de $\prod_{i \in I} X_{i}$ que é sup-finitamente distal com respeito a $\left\{d_{i}: i \in I\right\}$. Assumindo-se AC ou, supondo-se que, para cada $i \in I$, di seja uma métrica sobre $X_{i}$, tem-se que:

(i) $\left(d_{i}\right)_{I, \infty, A}$ é completa.

(ii) Para todo $\xi \in \prod_{i \in I} X_{i},\left(d_{i}\right)_{I, \infty, \xi \text { é completa. }}$

Prova. Suponha que se possa fixar uma sequência $s$ em $A$ tal que, para todo $i \in I$, a sequência $\langle(s(n))(i)\rangle_{n<\omega}$ em $X_{i}$ é convergente com respeito a $\tau_{d_{i}}$. Assim, tem-se que, para todo $i \in I$, o conjunto

$$
L_{i}:=\left\{x \in X_{i}:\langle(s(n))(i)\rangle_{n<\omega} \text { converge para } x \text { com respeito a } \tau_{d_{i}}\right\}
$$

\footnotetext{
${ }^{3}$ Lembrar que este termo é uma tradução da palavra francesa naïve, que teóricos conjuntistas utilizam com a conotação de algo despreocupado ou pouco cauteloso em relação ao rigor lógico da Teoria Axiomática dos Conjuntos.
} 
é não vazio. Considere então a família $\mathcal{L}_{s}:=\left\{L_{i}: i \in I\right\}$.

Assuma AC: fixe uma função escolha $\phi$ para $\mathcal{L}_{s}$ (i.e., uma função de $\mathcal{L}_{s}$ em $\cup \mathcal{L}_{s}$ tal que, para todo $i \in I$,

$$
\left.\phi\left(L_{i}\right) \in L_{i}\right)
$$

Considere a função $\xi_{s, \phi}: I \rightarrow \bigcup_{i \in I} X_{i}$ que é definida pondo-se, para todo $i \in I$,

$$
\xi_{s, \phi}(i):=\phi\left(L_{i}\right)
$$

É claro que $\xi_{s, \phi} \in \prod_{i \in I} X_{i}$ e que, para todo $i \in I,\langle(s(n))(i)\rangle_{n<\omega}$ converge para $\xi_{s, \phi}(i)$ com respeito a $\tau_{d_{i}}$.

Suponha que, para cada $i \in I, d_{i}$ seja uma métrica sobre $X_{i}$. Sendo assim, para todo $i \in I,\left\langle X_{i}, \tau_{d_{i}}\right\rangle$ é de Hausdorff (cf. Proposição 1.2.10(iii)), o que implica que existe um único limite de $\langle(s(n))(i)\rangle_{n<\omega}$ com respeito a $\tau_{d_{i}}$ (cf. Teorema 1.1.20(i)). Considere a função $\xi_{s}: I \rightarrow \bigcup_{i \in I} X_{i}$ que é definida pondo-se, para todo $i \in I$,

$$
\xi_{s}(i):=\lim _{n<\omega}(s(n))(i)
$$

É claro que $\xi_{s} \in \prod_{i \in I} X_{i}$ e que, para todo $i \in I,\langle(s(n))(i)\rangle_{n<\omega}$ converge para $\xi_{s}(i)$ com respeito a $\tau_{d_{i}}$.

(i) : Fixe arbitrariamente uma sequência $s$ em $A$ que é de Cauchy segundo $\left(d_{i}\right)_{I, \infty, A}$. Tome um $i \in I$ qualquer. Como, para quaisquer $n, m<\omega$,

$$
d_{i}((s(n))(i),(s(m))(i)) \leqslant\left(d_{i}\right)_{I, \infty, A}(s(n), s(m)),
$$

é imediato concluir que a sequência $\langle(s(n))(i)\rangle_{n<\omega}$ em $X_{i}$ é de Cauchy segundo $d_{i}$. Já que $d_{i}$ é completa, tem-se que $\langle(s(n))(i)\rangle_{n<\omega}$ é convergente com respeito a $\tau_{d_{i}}$.

Assuma AC ou suponha que, para cada $i \in I, d_{i}$ seja uma métrica sobre $X_{i}$ : fixe então um $\xi \in \prod_{i \in I} X_{i}$ tal que, para todo $i \in I,\langle(s(n))(i)\rangle_{n<\omega}$ converge para $\xi(i)$ com respeito a $\tau_{d_{i}}$. Como $s$ é limitada segundo $\left(d_{i}\right)_{I, \infty, A}$ (cf. Proposição 1.2.20(iii)), fixe um $\delta \in \mathbb{R}^{+}$tal que

$$
\operatorname{im}\left(\left(\left(d_{i}\right)_{I, \infty, A}\right)_{\operatorname{im}(s)}\right) \subseteq[0, \delta] .
$$

Tome um $i \in I$ qualquer. Já que a sequência $\left\langle d_{i}((s(n))(i), \xi(i))\right\rangle_{n<\omega}$ converge para 0 com respeito a $\tau_{2}$ (cf. Proposição 1.2.19(iv)), fixe um $k<\omega$ tal que

$$
d_{i}((s(k))(i), \xi(i))<1 .
$$


Então, tem-se que:

$$
\begin{aligned}
d_{i}(\xi(i),(s(0))(i)) & \leqslant d_{i}(\xi(i),(s(k))(i))+d_{i}((s(k))(i),(s(0))(i)) \leqslant \\
& \leqslant 1+\left(d_{i}\right)_{I, \infty, A}(s(k), s(0)) \leqslant 1+\delta .
\end{aligned}
$$

Logo,

$$
\left\{d_{i}(\xi(i),(s(0))(i)): i \in I\right\} \subseteq[0,1+\delta] .
$$

Assim, tem-se que $\xi$ está finitamente distante de $s(0)$ com respeito a $\left\{d_{i}: i \in I\right\}$, o que equivale a $\xi \in \ell_{\infty, s(0)}\left(I, d_{i}\right)$. Como $A$ é sup-finitamente distal com respeito a $\left\{d_{i}: i \in I\right\}$, fixe então uma função $\Lambda: \ell_{\infty, s(0)}\left(I, d_{i}\right) \rightarrow A$ que satisfaz a seguinte condição:

$$
\text { Para todo } n<\omega,\left(d_{i}\right)_{I, \infty, A}(\Lambda(\xi), s(n)) \leqslant\left(d_{i}\right)_{I, \infty, s(0)}(\xi, s(n)) \text {. }
$$

Tome um $\varepsilon \in \mathbb{R}^{+}$qualquer. Já que $s$ é de Cauchy segundo $\left(d_{i}\right)_{I, \infty, A}$, fixe um $k<\omega$ tal que, para todo $n, m \in \omega \backslash k$,

$$
\left(d_{i}\right)_{I, \infty, A}(s(n), s(m))<\frac{1}{4} \varepsilon .
$$

Tome um $i \in I$ qualquer. Como a sequência $\left\langle d_{i}((s(n))(i), \xi(i))\right\rangle_{n<\omega}$ converge para 0 com respeito a $\tau_{2}$, fixe um $l<\omega$ tal que

$$
d_{i}((s(l+k))(i), \xi(i))<\frac{1}{4} \varepsilon .
$$

Tome um $n \in \omega \backslash k$ qualquer. Assim, tem-se que:

$$
\begin{aligned}
d_{i}((s(n))(i), \xi(i)) & \leqslant d_{i}((s(n))(i),(s(l+k))(i))+d_{i}((s(l+k))(i), \xi(i)) \leqslant \\
& \leqslant\left(d_{i}\right)_{I, \infty, A}(s(n), s(l+k))+\frac{1}{4} \varepsilon \leqslant \frac{1}{4} \varepsilon+\frac{1}{4} \varepsilon=\frac{1}{2} \varepsilon .
\end{aligned}
$$

Logo,

$$
\left(d_{i}\right)_{I, \infty, s(0)}(\xi, s(n))=\left(d_{i}\right)_{I, \infty, s(0)}(s(n), \xi) \leqslant \frac{1}{2} \varepsilon<\varepsilon .
$$

Usando $(*)$, tem-se então que:

$$
\left(d_{i}\right)_{I, \infty, A}(s(n), \Lambda(\xi))=\left(d_{i}\right)_{I, \infty, A}(\Lambda(\xi), s(n)) \leqslant\left(d_{i}\right)_{I, \infty, s(0)}(\xi, s(n))<\varepsilon .
$$

Então, a sequência $\left(\left(d_{i}\right)_{I, \infty, A}\right)^{\Lambda(\xi)} \circ s$ converge para 0 com respeito a $\tau_{2}$, o que equivale a dizer que $s$ converge para $\Lambda(\xi)$ com respeito a $\tau_{\left(d_{i}\right)_{I, \infty, A}}$ (cf. Proposição 1.2.19(iv)). Portanto, conclui-se que $\left(d_{i}\right)_{I, \infty, A}$ é completa.

(ii) : Segue imediatamente dos itens (ii) (2) e (i) (1) da Proposição 2.2.8 e de (i) (1).

O próximo teorema é análogo ao Teorema 2.2.9, mas, na literatura a que tivemos acesso, sequer encontramos a sua versão restrita aos espaços métricos . 
Teorema 2.2.10. Sejam I um conjunto não vazio e $\mathcal{U}$ um ultrafiltro sobre I. Sejam $\left\{\left\langle X_{i}, d_{i}\right\rangle: i \in I\right\}$ uma família de espaços pseudométricos completos tal que o produto cartesiano $\prod_{i \in I} X_{i}$ é não vazio e A um subconjunto de $\prod_{i \in I} X_{i}$ que é $\mathcal{U}$-finitamente distal com respeito a $\left\{d_{i}: i \in I\right\}$. Assumindo-se AC ou, supondo-se que, para cada $i \in I, d_{i}$ seja uma métrica sobre $X_{i}$, tem-se que:

(i) $\left(d_{i}\right)_{I, \mathcal{U}, A}$ é completa.

(ii) Para todo $\xi \in \prod_{i \in I} X_{i},\left(d_{i}\right)_{I, \mathcal{U}, \xi e ́ \text { completa. }}$

Prova. Suponha que se possa fixar um $J \subseteq I$ e uma sequência s em $A$ tais que, para todo $i \in J$, a sequência $\langle(s(n))(i)\rangle_{n<\omega}$ em $X_{i}$ é convergente com respeito a $\tau_{d_{i}}$. Assim, tem-se que, para todo $i \in J$, o conjunto

$$
L_{i}:=\left\{x \in X_{i}:\langle(s(n))(i)\rangle_{n<\omega} \text { converge para } x \text { com respeito a } \tau_{d_{i}}\right\}
$$

é não vazio. Considere então a família $\mathcal{L}_{S, J}:=\left\{L_{i}: i \in J\right\}$.

Assuma AC: fixe uma função escolha $\phi$ para $\mathcal{L}_{S, J}$ (i.e., uma função de $\mathcal{L}_{s, J}$ em $\bigcup \mathcal{L}_{S, J}$ tal que, para todo $i \in J$,

$$
\left.\phi\left(L_{i}\right) \in L_{i}\right)
$$

Considere a função $\xi_{s, \phi}: J \rightarrow \bigcup_{i \in J} X_{i}$ que é definida pondo-se, para todo $i \in J$,

$$
\xi_{s, \phi}(i):=\phi\left(L_{i}\right)
$$

É claro que $\xi_{s, \phi} \in \prod_{i \in J} X_{i}$ e que, para todo $i \in J,\langle(s(n))(i)\rangle_{n<\omega}$ converge para $\xi_{s, \phi}(i)$ com respeito a $\tau_{d_{i}}$.

Suponha que, para cada $i \in I, d_{i}$ seja uma métrica sobre $X_{i}$. Sendo assim, para todo $i \in I,\left\langle X_{i}, \tau_{d_{i}}\right\rangle$ é de Hausdorff, o que implica que existe um único limite de $\langle(s(n))(i)\rangle_{n<\omega}$ com respeito a $\tau_{d_{i}}$. Considere a função $\xi_{s}: J \rightarrow \bigcup_{i \in J} X_{i}$ que é definida pondo-se, para todo $i \in J$,

$$
\xi_{s}(i):=\lim _{n<\omega}(s(n))(i)
$$

É claro que $\xi_{s} \in \prod_{i \in J} X_{i}$ e que, para todo $i \in J,\langle(s(n))(i)\rangle_{n<\omega}$ converge para $\xi_{s}(i)$ com respeito a $\tau_{d_{i}}$.

(i) : Fixe arbitrariamente uma sequência s em $A$ tal que, para todo $n<\omega$,

$$
\left(d_{i}\right)_{I, \mathcal{U}, A}(s(n), s(n+1))<2^{-(n+1)} \text {. }
$$


Para cada $n<\omega$, considere o seguinte conjunto:

$$
J_{n}:=\bigcap_{k<n+1}\left\{i \in I: d_{i}((s(k))(i),(s(k+1))(i))<2^{-(k+1)}\right\} .
$$

É claro que:

$$
\text { Para quaisquer } n, m<\omega \text {, se } n \leqslant m \text {, então } J_{m} \subseteq J_{n} \text {. }
$$

Além disso, tem-se que:

$$
\text { Para todo } n<\omega, J_{n} \in \mathcal{U} \text {. }
$$

Mostraremos que (**) vale: suponha que se possa fixar um $m<\omega$ tal que $J_{m} \notin \mathcal{U}$. Como $\mathcal{U}$ é um filtro sobre $I$, fixe um $l<m+1$ tal que

$$
\left\{i \in I: d_{i}((s(l))(i),(s(l+1))(i))<2^{-(l+1)}\right\} \notin \mathcal{U}
$$

Já que $\mathcal{U}$ é um ultrafiltro sobre $I$, segue que:

$$
\left\{i \in I: 2^{-(l+1)} \leqslant d_{i}((s(l))(i),(s(l+1))(i))\right\} \in \mathcal{U}
$$

Lembrando que $c_{I, \mathbb{R}, 2^{-(l+1)}}$ denota a função constante de valor $2^{-(l+1)}$ de $I$ em $\mathbb{R}$ (cf. Comentário 2.2.2), tem-se que $c_{I, \mathbb{R}, 2^{-(l+1)}} \leqslant \mathcal{U}\left\langle d_{i}((s(l))(i),(s(l+1))(i))\right\rangle_{i \in I}$. Logo,

$$
2^{-(l+1)}=\mathcal{U}-\lim c_{I, \mathbb{R}, 2^{-(l+1)}} \leqslant \mathcal{U}-\lim d_{i}((s(l))(i),(s(l+1))(i))=\left(d_{i}\right)_{I, \mathcal{U}, A}(s(l), s(l+1))
$$

(em virtude do Teorema 2.1.25 (i) e do Comentário 2.1.5(iv)(2)), uma contradição.

Para cada $i \in I$, considere o conjunto $M_{i}:=\left\{n<\omega: i \in J_{n} \backslash J_{n+1}\right\}$. Assim, tem-se que:

$$
\text { Para todo } i \in I, M_{i}=\emptyset \text { ou } M_{i} \text { é unitário. }
$$

Mostraremos que (†) vale: suponha que se possa fixar um $j \in I$ tal que $M_{j}$ não é vazio nem é unitário. Fixe então $k, l<\omega$ tais que $k \neq l, j \in J_{k} \backslash J_{k+1}$ e $j \in J_{l} \backslash J_{l+1}$. Tome $n_{k, l}:=\min \{k, l\}$ e $m_{k, l}:=\max \{k, l\}$. Como $n_{k, l}<m_{k, l}$, então $n_{k, l}+1 \leqslant m_{k, l}$. Usando (*), tem-se que $J_{m_{k, l}} \subseteq J_{n_{k, l}+1}$, uma contradição (pois $j \in J_{m_{k, l}}$ e $j \notin J_{n_{k, l}+1}$ ).

Tome um $i \in I$ qualquer. Suponha que $i \in J_{0}$ e que $M_{i}=\emptyset$. Suponha que se possa fixar um $n<\omega$ tal que $i \in J_{n}$. Já que $M_{i}=\emptyset$, segue que $i \notin J_{n}$ ou $i \in J_{n+1}$. Logo, $i \in J_{n+1}$. Por indução finita, conclui-se que, para todo $n<\omega, i \in J_{n}$. Então, tem-se que:

$$
\text { Para todo } i \in I \text {, se } i \in J_{0} \text { e } M_{i}=\emptyset \text {, então } i \in \bigcap_{n<\omega} J_{n} \text {. }
$$


Considere agora os conjuntos $J_{\mathrm{sng}}:=\left\{i \in I: M_{i}\right.$ é unitário $\}$ e $J_{\omega}:=\bigcap_{n<\omega} J_{n}$. É claro que $\left(I \backslash J_{0}\right) \cap J_{\omega}=\emptyset$. Note que $J_{\text {sng }} \cap J_{\omega}=\emptyset$. Como, para todo $n<\omega, J_{n} \subseteq J_{0}$, segue que $\left(I \backslash J_{0}\right) \cap J_{\text {sng }}=\emptyset$. Com o uso de $(\dagger)$ e $(\dagger+)$, conclui-se que:

$$
I=\left(I \backslash J_{0}\right) \cup J_{\text {sng }} \cup J_{\omega} \text {. }
$$

Para cada $i \in J_{\mathrm{sng}}$, denote por $n_{i}$ o único elemento de $M_{i}$. Tome um $i \in J_{\omega}$ qualquer. Assim, tem-se que, para todo $n<\omega$,

$$
d_{i}((s(n))(i),(s(n+1))(i))<2^{-(n+1)} .
$$

Logo, a sequência $\langle(s(n))(i)\rangle_{n<\omega}$ em $X_{i}$ é de Cauchy segundo $d_{i}$ (cf. Teorema 1.2.21 (iii)). Já que $d_{i}$ é completa, tem-se que $\langle(s(n))(i)\rangle_{n<\omega}$ é convergente com respeito a $\tau_{d_{i}}$.

Assuma AC ou suponha que, para cada $i \in I, d_{i}$ seja uma métrica sobre $X_{i}$ : fixe então um $\xi \in \prod_{i \in J_{\omega}} X_{i}$ tal que,

(†) $\quad$ para todo $i \in J_{\omega},\langle(s(n))(i)\rangle_{n<\omega}$ converge para $\xi(i)$ com respeito a $\tau_{d_{i}}$.

Agora, considere a função $\xi_{\omega}: I \rightarrow \bigcup_{i \in I} X_{i}$ que é definida pondo-se, para todo $i \in I$,

$$
\xi_{\omega}(i):=\left\{\begin{aligned}
(s(0))(i), & \text { se } i \in I \backslash J_{0} \\
\left(s\left(n_{i}+1\right)\right)(i), & \text { se } i \in J_{\mathrm{sng}} \\
\xi(i), & \text { se } i \in J_{\omega} .
\end{aligned}\right.
$$

É claro que $\xi_{\omega} \in \prod_{i \in J} X_{i}$. Além disso, tem-se que:

$$
\text { Para todo } n<\omega, J_{n} \subseteq\left\{i \in I: d_{i}\left((s(n))(i), \xi_{\omega}(i)\right) \leqslant 2^{-n+1}\right\}
$$

Mostraremos que (ł‡) vale: fixe arbitrariamente um $n<\omega$ e um $i \in J_{n}$. Como $J_{n} \subseteq J_{0}$ ， então $i \notin I \backslash J_{0}$. Há dois casos a considerar:

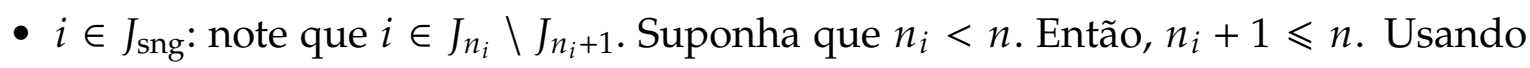
(*), conclui-se que $J_{n} \subseteq J_{n_{i}+1}$, uma contradição. Logo, $n \leqslant n_{i}$. Já que, para todo $k<n_{1}+1$,

$$
d_{i}((s(k))(i),(s(k+1))(i))<2^{-(k+1)}=\left(2^{-1}\right)^{k+1}
$$

tem-se que:

$$
\begin{aligned}
d_{i}\left((s(n))(i), \xi_{\omega}(i)\right) & =d_{i}\left((s(n))(i),\left(s\left(n_{i}+1\right)\right)(i)\right)<\left(1-2^{-1}\right)^{-1}\left(2^{-1}\right)^{n+1}=2^{-n}< \\
& <2^{-n+1} .
\end{aligned}
$$

(cf. Proposição 1.2.3). 
- $i \in J_{\omega}$ : usando (†), fixe um $k<\omega$ tal que

$$
d_{i}\left((s(k+n))(i), \xi_{\omega}(i)\right)=d_{i}((s(k+n))(i), \xi(i))<2^{-n} .
$$

Agora, note que, para todo $m<\omega$,

$$
d_{i}((s(m))(i),(s(m+1))(i))<2^{-(m+1)}=\left(2^{-1}\right)^{m+1} .
$$

Então, tem-se que:

$$
d_{i}((s(n))(i),(s(k+n))(i))<\left(1-2^{-1}\right)^{-1}\left(2^{-1}\right)^{n+1}=2^{-n}
$$

(cf. Corolário 1.2.4). Logo,

$$
\begin{aligned}
d_{i}\left((s(n))(i), \xi_{\omega}(i)\right) & \leqslant d_{i}((s(n))(i),(s(k+n))(i))+d_{i}\left((s(k+n))(i), \xi_{\omega}(i)\right)< \\
& <2^{-n}+2^{-n}=2^{-n+1} .
\end{aligned}
$$

Usando (†‡), conclui-se que:

$$
\begin{aligned}
& \left\{d_{i}\left(\xi_{\omega}(i),(s(0))(i)\right): i \in I\right\}=\left\{d_{i}\left((s(0))(i), \xi_{\omega}(i)\right): i \in I\right\}= \\
= & \left\{d_{i}\left((s(0))(i), \xi_{\omega}(i)\right): i \in J_{0}\right\} \cup\left\{d_{i}\left((s(0))(i), \xi_{\omega}(i)\right): i \in I \backslash J_{0}\right\}= \\
= & {\left[0,2^{-0+1}\right] \cup\{0\} \subseteq[0,2] . }
\end{aligned}
$$

Então, $\xi_{\omega}$ está finitamente distante de $s(0)$ com respeito a $\left\{d_{i}: i \in I\right\}$, o que equivale a $\xi_{\omega} \in \ell_{\infty, s(0)}\left(I, d_{i}\right)$. Como $A$ é $\mathcal{U}$-finitamente distal com respeito a $\left\{d_{i}: i \in I\right\}$, fixe então uma função $\Phi: \ell_{\infty, s(0)}\left(I, d_{i}\right) \rightarrow A$ que satisfaz a seguinte condição:

$$
\text { Para todo } n<\omega,\left(d_{i}\right)_{I, \mathcal{U}, A}\left(\Phi\left(\xi_{\omega}\right), s(n)\right) \leqslant\left(d_{i}\right)_{I, \mathcal{U}, s(0)}\left(\xi_{\omega}, s(n)\right) \text {. }
$$

Fixe arbitrariamente um $n<\omega$. Já que $\mathcal{U}$ é um filtro sobre $I$, conclui-se com o uso de (**) e (†‡) que:

$$
\left\{i \in I: d_{i}\left((s(n))(i), \xi_{\omega}(i)\right) \leqslant 2^{-n+1}\right\} \in \mathcal{U},
$$

o que equivale a $\left\langle d_{i}\left((s(n))(i), \xi_{\omega}(i)\right)\right\rangle_{i \in I} \leqslant \mathcal{U} c_{I, \mathbb{R}, 2^{-n+1}}$. Assim, tem-se que:

$$
\left(d_{i}\right)_{I, \mathcal{U}, s(0)}\left(s(n), \xi_{\omega}\right)=\mathcal{U}-\lim d_{i}\left((s(n))(i), \xi_{\omega}(i)\right) \leqslant \mathcal{U}-\lim c_{I, \mathbb{R}, 2^{-n+1}}=2^{-n+1} .
$$

Usando (\#), tem-se então que:

$$
\left(d_{i}\right)_{I, \mathcal{U}, A}\left(\Phi\left(\xi_{\omega}\right), s(n)\right) \leqslant 2^{-n+1} .
$$

Tome um $\varepsilon \in \mathbb{R}^{+}$qualquer. Utilizando a propriedade arquimediana do corpo ordenado $\mathbb{R}$, fixe um $k<\omega$ tal que $2^{-k+1}<\varepsilon$. Logo, para todo $n \in \omega \backslash k$,

$$
\left(d_{i}\right)_{I, \mathcal{U}, A}\left(s(n), \Phi\left(\xi_{\omega}\right)\right)=\left(d_{i}\right)_{I, \mathcal{U}, A}\left(\Phi\left(\xi_{\omega}\right), s(n)\right) \leqslant 2^{-n+1} \leqslant 2^{-k+1}<\varepsilon
$$


Então, a sequência $\left(\left(d_{i}\right)_{I, \mathcal{U}, A}\right)^{\Phi\left(\xi_{\omega}\right)} \circ s$ converge para 0 com respeito a $\tau_{2}$, o que equivale a dizer que $s$ converge para $\Phi\left(\xi_{\omega}\right)$ com respeito a $\tau_{\left(d_{i}\right)_{I, \mathcal{U}} A}$ (cf. Proposição 1.2.19(iv)). Em virtude do Corolário 1.2.24, conclui-se que $\left(d_{i}\right)_{I, \mathcal{U}, A}$ é completa.

(ii) : Segue imediatamente dos itens (ii) (2) e (i) (2) da Proposição 2.2.8 e de (i) (1).

Vale salientar que a prova do item (i) do Teorema 2.2.10 foi inspirada no esboço de prova da Proposição 5.3 de [B $\left.{ }^{+} 2008\right]$.

O teorema a seguir tem dois resultados análogos aos da Proposição 2.2.5, os quais são generalizações nossas de outros que estão demonstrados no contexto dos espaços normados.

Teorema 2.2.11. Sejam I um conjunto não vazio e $\mathcal{U}$ um ultrafiltro sobre I. Sejam $\left\{\left\langle X_{i}, d_{i}\right\rangle: i \in I\right\}$ uma família de espaços pseudométricos tal que o produto cartesiano $\prod_{i \in I} X_{i}$ é não vazio e A um subconjunto de $\prod_{i \in I} X_{i}$ que é $\mathcal{U}$-sup-finitamente distal com respeito a $\left\{d_{i}: i \in I\right\}$. Então:

(i) (1) Para quaisquer $\zeta, \zeta^{\prime} \in A,\left(d_{i}\right)_{I, \mathcal{U}, A}\left(\zeta, \zeta^{\prime}\right)=\left(\left(d_{i}\right)_{I, \infty, A}\right)^{\mathcal{c}_{0}, \mathcal{U}, \zeta^{\prime}, A}\left(I, X_{i}, d_{i}\right)(\zeta)$.

(2) Para todo $\xi \in \prod_{i \in I} X_{i}$ e quaisquer $\zeta, \zeta^{\prime} \in \ell_{\infty, \xi}\left(I, X_{i}, d_{i}\right)$,

$$
\left(d_{i}\right)_{I, \mathcal{U}, \xi}\left(\zeta, \zeta^{\prime}\right)=\left(\left(d_{i}\right)_{I, \infty, \xi}\right)^{c_{0, \mathcal{U}, \zeta^{\prime}}\left(I, X_{i}, d_{i}\right)}(\zeta)
$$

(ii) (1) Para todo $\zeta \in A, c_{0, \mathcal{U}, \zeta, A}\left(I, X_{i}, d_{i}\right)$ é um fechado em $\left\langle A, \tau_{\left(d_{i}\right)_{I, \infty, A}}\right\rangle$.

(2) Para todo $\xi \in \prod_{i \in I} X_{i}, c_{0, \mathcal{U}, \xi}\left(I, X_{i}, d_{i}\right)$ é um fechado em $\left\langle\ell_{\infty, \xi}\left(I, X_{i}, d_{i}\right), \tau_{\left(d_{i}\right)_{I, \infty, \xi}}\right\rangle$.

Prova. (i) (1) : Fixe arbitrariamente $\zeta, \zeta^{\prime} \in A$. Para cada $\varepsilon^{\prime} \in \mathbb{R}^{+}$e cada $\eta \in A$, considere os seguintes conjuntos:

$$
\begin{aligned}
J_{\varepsilon^{\prime}} & :=\left\{i \in I: d_{i}\left(\zeta(i), \zeta^{\prime}(i)\right) \in\right]\left(d_{i}\right)_{I, \mathcal{U}, A}\left(\zeta, \zeta^{\prime}\right)-\frac{1}{2} \varepsilon^{\prime},\left(d_{i}\right)_{I, \mathcal{U}, A}\left(\zeta, \zeta^{\prime}\right)+\frac{1}{2} \varepsilon^{\prime}[\} \\
& \mathrm{e} \\
J_{\eta, \varepsilon^{\prime}} & :=\left\{i \in I: d_{i}\left(\eta(i), \zeta^{\prime}(i)\right) \in\left[0, \frac{1}{2} \varepsilon^{\prime}[\} .\right.\right.
\end{aligned}
$$

Tome um $\varepsilon \in \mathbb{R}^{+}$qualquer. Note que $J_{\varepsilon} \in \mathcal{U}$. Tome um $\eta \in c_{0, \mathcal{U}, \zeta^{\prime}, A}\left(I, d_{i}\right)$ qualquer. Já que

$$
J_{\eta, \varepsilon}=\left\{i \in I: d_{i}(\eta(i), \zeta(i)) \in\right]-\frac{1}{2} \varepsilon, \frac{1}{2} \varepsilon[\}
$$


então $J_{\eta, \varepsilon} \in \mathcal{U}$. Como $\mathcal{U}$ é um filtro sobre $I$, então $J_{\varepsilon} \cap J_{\eta, \varepsilon} \in \mathcal{U}$ e, por conseguinte, o conjunto $J_{\varepsilon} \cap J_{\eta, \varepsilon}$ é não vazio. Fixe um $j \in J_{\varepsilon} \cap J_{\eta, \varepsilon}$. Logo,

$$
\begin{aligned}
\left(d_{i}\right)_{I, \mathcal{U}, \xi}\left(\zeta, \zeta^{\prime}\right)-\varepsilon & =\left(\left(d_{i}\right)_{I, \mathcal{U}, \xi}\left(\zeta, \zeta^{\prime}\right)-\frac{1}{2} \varepsilon\right)-\frac{1}{2} \varepsilon<d_{j}\left(\zeta(j), \zeta^{\prime}(j)\right)-d_{j}\left(\eta(j), \zeta^{\prime}(j)\right) \leqslant \\
& \leqslant\left(d_{j}(\zeta(j), \eta(j))+d_{j}\left(\eta(j), \zeta^{\prime}(j)\right)\right)-d_{j}\left(\eta(j), \zeta^{\prime}(j)\right)= \\
& =d_{j}(\zeta(j), \eta(j)) \leqslant\left(d_{i}\right)_{I, \infty, \xi}(\zeta, \eta) .
\end{aligned}
$$

Já que $\eta \in c_{0, \mathcal{U}, \zeta^{\prime}, A}\left(I, d_{i}\right)$ foi tomado qualquer, segue que:

$(*) \quad\left(d_{i}\right)_{I, \mathcal{U}, A}\left(\zeta, \zeta^{\prime}\right)-\varepsilon \leqslant\left(d_{i}\right)_{I, \infty, A}\left(\zeta, c_{0, \mathcal{U}, \zeta^{\prime}, A}\left(I, d_{i}\right)\right)=\left(\left(d_{i}\right)_{I, \infty, A}\right)^{\mathcal{C}_{0, \mathcal{U}, \zeta^{\prime}, A}\left(I, d_{i}\right)}(\zeta)$.

Considere a função $\zeta_{\varepsilon}: I \rightarrow \bigcup_{i \in I} X_{i}$ que é definida pondo-se, para todo $i \in I$,

$$
\zeta_{\varepsilon}(i):= \begin{cases}\zeta^{\prime}(i), & \text { se } i \in J_{\varepsilon} \\ \zeta(i), & \text { caso contrário. }\end{cases}
$$

É claro que $\zeta_{\varepsilon} \in \prod_{i \in I} X_{i}$. Tome $\delta_{\zeta, \zeta^{\prime}}:=\left(d_{i}\right)_{I, \infty, A}\left(\zeta, \zeta^{\prime}\right)+1$. Sendo assim, $\delta_{\zeta, \zeta^{\prime}} \in \mathbb{R}^{+} \mathrm{e}$

$$
\begin{aligned}
\left\{d_{i}\left(\zeta_{\varepsilon}(i), \zeta^{\prime}(i)\right): i \in I\right\} & =\left\{d_{i}\left(\zeta_{\varepsilon}(i), \zeta^{\prime}(i)\right): i \in J_{\varepsilon}\right\} \cup\left\{d_{i}\left(\zeta_{\varepsilon}(i), \zeta^{\prime}(i)\right): i \in I \backslash J_{\varepsilon}\right\}= \\
& =\{0\} \cup\left\{d_{i}\left(\zeta(i), \zeta^{\prime}(i)\right): i \in I \backslash J_{\varepsilon}\right\} \subseteq\left[0, \delta_{\zeta, \zeta^{\prime}}\right]
\end{aligned}
$$

Então, $\zeta_{\varepsilon}$ está finitamente distante de $\zeta^{\prime}$ com respeito a $\left\{d_{i}: i \in I\right\}$, o que equivale a $\zeta_{\varepsilon} \in \ell_{\infty, \zeta^{\prime}}\left(I, d_{i}\right)$. Tome um $\varepsilon^{\prime} \in \mathbb{R}^{+}$qualquer. Note que $J_{\varepsilon} \subseteq J_{\zeta_{\varepsilon}, \varepsilon^{\prime}}$. Como $\mathcal{U}$ é um filtro sobre $I$, segue que $J_{\zeta_{\varepsilon}, \varepsilon^{\prime}} \in \mathcal{U}$, o que equivale a dizer que a família $\left\langle d_{i}\left(\zeta_{\varepsilon}(i), \zeta^{\prime}(i)\right)\right\rangle_{i \in I}$ está $\mathcal{U}$-eventualmente em $]-\varepsilon^{\prime}, \varepsilon^{\prime}\left[=B_{d_{2}}\left(0, \varepsilon^{\prime}\right)\right.$. Já que $\varepsilon^{\prime} \in \mathbb{R}^{+}$foi tomado qualquer, tem-se que:

$$
\mathcal{U}-\lim d_{i}\left(\zeta_{\varepsilon}(i), \zeta^{\prime}(i)\right)=\mathcal{U}-\lim d_{2}\left(d_{i}\left(\zeta_{\varepsilon}(i), \zeta^{\prime}(i)\right), 0\right)=0
$$

(cf. Corolário 2.1.6 (i)), o que implica que

$$
\left(d_{i}\right)_{I, \mathcal{U}, \zeta^{\prime}}\left(\zeta_{\varepsilon}, \zeta^{\prime}\right)=0
$$

Como $A$ é finitamente distal com respeito a $\left\{d_{i}: i \in I\right\}$, tem-se que $\zeta$ está finitamente distante de $\zeta^{\prime}$ com respeito a $\left\{d_{i}: i \in I\right\}$, o que equivale a $\zeta \in \ell_{\infty, \zeta^{\prime}}\left(I, d_{i}\right)$. Além disso, tem-se que:

$$
\begin{aligned}
\left\{d_{i}\left(\zeta_{\varepsilon}(i), \zeta(i)\right): i \in I\right\} & =\left\{d_{i}\left(\zeta(i), \zeta_{\varepsilon}(i)\right): i \in I\right\}= \\
& =\left\{d_{i}\left(\zeta(i), \zeta_{\varepsilon}(i)\right): i \in J_{\varepsilon}\right\} \cup\left\{d_{i}\left(\zeta(i), \zeta_{\varepsilon}(i)\right): i \in I \backslash J_{\varepsilon}\right\}= \\
& =\left\{d_{i}\left(\zeta(i), \zeta^{\prime}(i)\right): i \in J_{\varepsilon}\right\} \cup\{0\} \subseteq\left[0,\left(d_{i}\right)_{I, \mathcal{U}, A}\left(\zeta, \zeta^{\prime}\right)+\varepsilon[,\right.
\end{aligned}
$$


o que implica que

$$
\left(d_{i}\right)_{I, \infty, \zeta^{\prime}}\left(\zeta_{\varepsilon}, \zeta^{\prime}\right) \leqslant\left(d_{i}\right)_{I, \mathcal{U}, A}\left(\zeta, \zeta^{\prime}\right)+\varepsilon
$$

Já que $A$ é $\mathcal{U}$-sup-finitamente distal com respeito a $\left\{d_{i}: i \in I\right\}$, fixe então uma função $\Psi: \ell_{\infty, \zeta^{\prime}}\left(I, d_{i}\right) \rightarrow A$ que satisfaz as seguintes condições:

$$
\begin{gathered}
\left(d_{i}\right)_{I, \infty, A}\left(\Psi\left(\zeta_{\varepsilon}\right), \zeta\right) \leqslant\left(d_{i}\right)_{I, \infty, \zeta^{\prime}}\left(\zeta_{\varepsilon}, \zeta\right) \\
\left(d_{i}\right)_{I, \mathcal{U}, A}\left(\Psi\left(\zeta_{\varepsilon}\right), \zeta^{\prime}\right) \leqslant\left(d_{i}\right)_{I, \mathcal{U}, \zeta^{\prime}}\left(\zeta_{\varepsilon}, \zeta^{\prime}\right) .
\end{gathered}
$$

Com o uso de $(\dagger)$ e $(\ddagger \ddagger)$, conclui-se que $\left(d_{i}\right)_{I, \mathcal{U}, A}\left(\Psi\left(\zeta_{\varepsilon}\right), \zeta^{\prime}\right)=0$, o que equivale a

$$
\Psi\left(\zeta_{\varepsilon}\right) \in c_{0, \mathcal{U}, \zeta^{\prime}}\left(I, d_{i}\right)
$$

Com o uso de $(\dagger+)$ e $(\ddagger)$, conclui-se que:

$$
\left(d_{i}\right)_{I, \mathcal{U}, A}\left(\Psi\left(\zeta_{\varepsilon}\right), \zeta^{\prime}\right) \leqslant\left(d_{i}\right)_{I, \mathcal{U}, A}\left(\zeta, \zeta^{\prime}\right)+\varepsilon
$$

Logo,

$(* *) \quad\left(\left(d_{i}\right)_{I, \infty, A}\right)^{\mathcal{c}_{0, \mathcal{U}, \zeta^{\prime}, A}\left(I, d_{i}\right)}(\zeta)=\left(d_{i}\right)_{I, \infty, A}\left(\zeta, c_{0, \mathcal{U}, \zeta^{\prime}, A}\left(I, d_{i}\right)\right) \leqslant\left(d_{i}\right)_{I, \mathcal{U}, A}\left(\zeta, \zeta^{\prime}\right)+\varepsilon$.

Em virtude de $(*)$ e $(* *)$, tem-se que:

$$
\left|\left(\left(d_{i}\right)_{I, \infty, A}\right)^{c_{0}, \mathcal{U}, \zeta^{\prime}, A}\left(I, d_{i}\right)(\zeta)-\left(d_{i}\right)_{I, \mathcal{U}, A}\left(\zeta, \zeta^{\prime}\right)\right| \leqslant \varepsilon
$$

Portanto, como se tomou $\varepsilon \in \mathbb{R}^{+}$qualquer, conclui-se que:

$$
\left(d_{i}\right)_{I, \mathcal{U}, A}\left(\zeta, \zeta^{\prime}\right)=\left(\left(d_{i}\right)_{I, \infty, A}\right)^{\mathcal{C}_{0}, \mathcal{U}, \zeta^{\prime}, A}\left(I, X_{i}, d_{i}\right)(\zeta)
$$

(i) (2) : Segue imediatamente da Proposição 2.2.8 (ii) (2) e de (i) (1).

(ii)(2) : Em virtude de (i)(1) e do Comentário 1.2.9(v)(2)(1), tem-se que, para todo $\zeta \in A$,

$$
\begin{aligned}
\overline{c_{0, \mathcal{U}, \zeta, A}\left(I, d_{i}\right)} \tau^{\tau_{\left(d_{i}\right)_{I, \infty, A}}} & =\left(\left(\left(d_{i}\right)_{I, \infty, A}\right)^{c_{0, \mathcal{U}, \zeta, A}\left(I, d_{i}\right)}\right)^{-1}[\{0\}]= \\
& =\left\{\eta \in A:\left(\left(d_{i}\right)_{I, \infty, A}\right)^{\mathcal{C}_{0, \mathcal{U}, \zeta, A}\left(I, d_{i}\right)}(\eta)=0\right\}= \\
& =\left\{\eta \in A:\left(d_{i}\right)_{I, \mathcal{U}, A}(\eta, \zeta)=0\right\}=c_{0, \mathcal{U}, \zeta, A}\left(I, d_{i}\right) .
\end{aligned}
$$

(ii) (2) : É imediato de (ii) (1).

A definição a seguir é uma generalização da definição de ultraproduto de espaços métricos, que se encontra, por exemplo, em [B+2008, p. 338-339]. 
Definição 2.2.12. Sejam $I$ um conjunto não vazio e $\mathcal{U}$ um ultrafiltro sobre $I$. Sejam $\left\{\left\langle X_{i}, d_{i}\right\rangle: i \in I\right\}$ uma família de espaços pseudométricos tal que o produto cartesiano $\prod_{i \in I} X_{i}$ é não vazio, $A$ um subconjunto de $\prod_{i \in I} X_{i}$ que é finitamente distal com respeito a $\left\{d_{i}: i \in I\right\}$ e $\alpha \in \prod_{i \in I} X_{i}$. Seja $\langle X, d\rangle$ um espaço pseudométrico tal que $X$ é não vazio. Denotaremos por $\prod_{I, d_{i}, \mathcal{U}, A} X_{i}$ o conjunto $A / R^{\left(d_{i}\right)_{I, \mathcal{U}, A}}$ e por $\bigotimes_{I, \mathcal{U}, A} d_{i}$ a métrica $\left(d_{i}\right)_{I, \mathcal{U}, A} / R^{\left(d_{i}\right)_{I, \mathcal{U}, A}}$. O espaço métrico $\left\langle A,\left(d_{i}\right)_{I, \mathcal{U}, A}\right\rangle / R^{\left(d_{i}\right)_{I}, \mathcal{U}, A}$ será denotado por $\prod_{I, \mathcal{U}, A}\left\langle X_{i}, d_{i}\right\rangle$. Diremos que:

(i) $\prod_{I, \mathcal{U}, A}\left\langle X_{i}, d_{i}\right\rangle$ é o ultraproduto (métrico) de $\left\{\left\langle X_{i}, d_{i}\right\rangle: i \in I\right\}$ com respeito a $\mathcal{U}$ e ancorado em $A$.

Denotaremos por $\prod_{I, d_{i}, \mathcal{U}, \alpha} X_{i}$ o conjunto $\prod_{I, d_{i}, \mathcal{U}, \ell_{\infty, \alpha}\left(I, X_{i}, d_{i}\right)} X_{i}$ e por $\bigotimes_{I, \mathcal{U}, \alpha} d_{i}$ a métrica $\bigotimes_{I, \mathcal{U}, \ell_{\infty, \alpha}\left(I, X_{i}, d_{i}\right)} d_{i}$. Oespaço métrico $\prod_{I, \mathcal{U}, \ell_{\infty, \alpha}\left(I, X_{i}, d_{i}\right)}\left\langle X_{i}, d_{i}\right\rangle$ será denotado por $\prod_{I, \mathcal{U}, \alpha}\left\langle X_{i}, d_{i}\right\rangle$. Diremos que:

(ii) $\prod_{I, \mathcal{U}, \alpha}\left\langle X_{i}, d_{i}\right\rangle$ é o ultraproduto (métrico) de $\left\{\left\langle X_{i}, d_{i}\right\rangle: i \in I\right\}$ com respeito a $\mathcal{U}$ e ancorado em $\alpha$.

Suponhamos que, para cada $i \in I,\left\langle X_{i}, d_{i}\right\rangle=\langle X, d\rangle$. Denotaremos por $X^{I, d}, \mathcal{U}, A$ (ou, simplesmente, por $X^{d, \mathcal{U}, A}$ ) o conjunto $\prod_{I, d_{i}, \mathcal{U}, A} X_{i}$ e por $d^{I, \mathcal{U}, A}$ (ou, simplesmente, por $\left.d^{\mathcal{U}}, A\right)$ a métrica $\bigotimes_{I, \mathcal{U}, A} d_{i}$. O espaço métrico $\prod_{I, \mathcal{U}, A}\left\langle X_{i}, d_{i}\right\rangle$ será denotado por $\langle X, d\rangle^{I, \mathcal{U}, A}$ (ou, simplesmente, por $\langle X, d\rangle^{\mathcal{U}, A}$ ). Diremos que:

(iii) $\langle X, d\rangle^{I, \mathcal{U}, A}$ é a ultrapotência (métrica) de $\langle X, d\rangle$ com respeito a $\mathcal{U}$ e ancorada $\operatorname{em} A$.

Denotaremos por $X^{I, d, \mathcal{U}, \alpha}$ (ou, simplesmente, por $\left.X^{d, \mathcal{U}, \alpha}\right)$ o conjunto $X^{I, d, \mathcal{U}, \ell_{\infty, \alpha}\left(I, X_{i}, d_{i}\right)}$ e por $d^{I, \mathcal{U}, \alpha}$ (ou, simplesmente, por $\left.d^{\mathcal{U}, \alpha}\right)$ a métrica $d^{I, \mathcal{U}, \ell_{\infty, \alpha}\left(I, X_{i}, d_{i}\right)}$. O espaço métrico $\langle X, d\rangle^{I, \mathcal{U}, \ell_{\infty, \alpha}(I, X, d)}$ será denotado por $\langle X, d\rangle^{I, \mathcal{U}, \alpha}$ (ou, simplesmente, por $\langle X, d\rangle^{\mathcal{U}, \alpha}$ ). Diremos que:

(iv) $\langle X, d\rangle^{I, \mathcal{U}, \alpha}$ é a ultrapotência (métrica) de $\langle X, d\rangle$ com respeito a $\mathcal{U}$ e ancorada em $\alpha$. 
Com o uso do Teorema 2.2.6 e do Comentário 1.2.12 (x) (2), conclui-se facilmente o corolário a seguir, que é uma generalização nossa do resultado (aparentemente) folclórico que afirma que

o conjunto suporte do ultraproduto de uma família de espaços métricos discretos pontuados é o ultraproduto da família de todos os conjuntos suportes desses espaços.

Corolário 2.2.13. Sejam I um conjunto não vazio e $\mathcal{U}$ um ultrafiltro sobre I. Sejam $\left\{X_{i}: i \in I\right\}$ uma família de conjuntos tal que o produto cartesiano $\prod_{i \in I} X_{i}$ é não vazio e A um subconjunto não vazio de $\prod_{i \in I} X_{i}$. Então:

(i) (1) A é finitamente distal com respeito a $\left\{d_{1, X_{i}}: i \in I\right\}$.

(2) $\prod_{I, \mathcal{U}, A}\left\langle X_{i}, d_{1, X_{i}}\right\rangle=\left\langle A /(\equiv \mathcal{U})_{A}, d_{1, A} /(\equiv \mathcal{u})_{A}\right\rangle$.

(ii) Para todo $\xi \in \prod_{i \in I} X_{i}$

(1) $\ell_{\infty, \xi}\left(I, X_{i}, d_{1, X_{i}}\right)=\prod_{i \in I} X_{i}$

(2) $\prod_{I, \mathcal{U}, \xi}\left\langle X_{i}, d_{1, X_{i}}\right\rangle=\left\langle\prod_{i \in I} X_{i} / \mathcal{U}, d_{1,} \prod_{i \in I} x_{i} / \mathcal{U}\right\rangle$.

O enunciado do teorema a seguir é obtido por uma simples adaptação do resultado (aparentemente) folclórico que afirma que

o ultraproduto de uma família de espaços métricos pontuados com respeito a um ultrafiltro principal sobre o conjunto de índices dessa família é isométrico a um desses espaços.

Teorema 2.2.14. Dados um conjunto não vazio $I$, um $j \in I$ e uma família $\left\{\left\langle X_{i}, d_{i}\right\rangle: i \in I\right\}$ de espaços pseudométricos tal que o produto cartesiano $\prod_{i \in I} X_{i}$ é não vazio, se $d_{j}$ for uma métrica sobre $X_{j}$, então, para todo $\xi \in \prod_{i \in I} X_{i}, \prod_{I, \mathcal{U}(j), \xi}\left\langle X_{i}, d_{i}\right\rangle$ é isométrico a $\left\langle X_{j}, d_{j}\right\rangle$.

Prova. Inicialmente, lembre-se que $p_{j}$ está denotando a projeção $p_{I, X_{i}, j}$ (v. Comentário 1.1.23) e que $\mathcal{U}(j)$ denota o ultrafiltro principal sobre $I$ em $j$ (cf. Comentário 1.1.2). 
Fixe arbitrariamente um $\xi \in \prod_{i \in I} X_{i}$. Considere a função $\varphi:=p_{j}\left\lceil\ell_{\infty, \xi}\left(I, d_{i}\right)\right.$. Então, para quaisquer $\zeta, \zeta^{\prime} \in \ell_{\infty, \xi}\left(I, d_{i}\right)$,

$$
\left(d_{i}\right)_{I, \mathcal{U}(j), \xi}\left(\zeta, \zeta^{\prime}\right)=\mathcal{U}-\lim d_{i}\left(\zeta(i), \zeta^{\prime}(i)\right)=d_{j}\left(\zeta(j), \zeta^{\prime}(j)\right)=d_{j}\left(\varphi(\zeta), \varphi\left(\zeta^{\prime}\right)\right)
$$

(v. Comentário 2.1.5 (v)). Logo, $\varphi$ preserva distância segundo $\left(d_{i}\right)_{I, \mathcal{U}(j), \xi}$ e $d_{j}$. Tome um $x \in X_{j}$ qualquer. Considere agora a função $\xi_{x}: I \rightarrow \bigcup_{i \in I} X_{i}$ que é definida pondo-se, para todo $i \in I$,

$$
\xi_{x}(i):=\left\{\begin{aligned}
x, & \text { se } i=j \\
\xi(i), & \text { caso contrário. }
\end{aligned}\right.
$$

É claro que $\xi_{x} \in \prod_{i \in I} X_{i}$. Tome $\delta_{x}:=d_{j}(x, \xi(j))+1$. Sendo assim, $\delta_{x} \in \mathbb{R}^{+} \mathrm{e}$

$$
\begin{aligned}
\left\{d_{i}\left(\xi_{x}(i), \xi(i)\right): i \in I\right\} & =\left\{d_{j}\left(\xi_{x}(j), \xi(j)\right)\right\} \cup\left\{d_{i}\left(\xi_{x}(i), \xi(i)\right): i \in I \backslash\{j\}\right\}= \\
& =\left\{d_{j}(x, \xi(j))\right\} \cup\{0\} \subseteq\left[0, \delta_{x}\right] .
\end{aligned}
$$

Então, $\xi_{x}$ está finitamente distante de $\xi$ com respeito a $\left\{d_{i}: i \in I\right\}$, o que equivale a $\xi_{x} \in \ell_{\infty, \xi}\left(I, d_{i}\right)$. Note que $\varphi\left(\xi_{x}\right)=x$. Já que $x \in X_{j}$ foi tomado qualquer, tem-se que $\varphi$ é sobrejetora. Portanto, supondo-se que $d_{j}$ seja uma métrica sobre $X_{j}$, conclui-se usando o Teorema 1.2.13(ii) (2) que $\prod_{I, \mathcal{U}(j), \xi}\left\langle X_{i}, d_{i}\right\rangle$ é isométrico a $\left\langle X_{j}, d_{j}\right\rangle$.

O próximo teorema é uma generalização nossa do resultado (aparentemente) folclórico que afirma que

um espaço métrico pontuado é isométrico a cada uma de suas ultrapotências se a topologia induzida por sua métrica for compacta.

Teorema 2.2.15. Sejam I um conjunto não vazio e $\mathcal{U}$ um ultrafiltro sobre I. Sejam $\langle X, d\rangle$ um espaço métrico de Heine-Borel tal que X é não vazio e A um subconjunto de $X^{I}$ que é finitamente distal segundo d e que contém qualquer constante em X. Então:

(i) Para todo $\xi \in A$, existe um único $\mathcal{U}$-limite de $\xi$ com respeito a $\tau_{d}$.

(ii) (1) $\langle X, d\rangle^{I, \mathcal{U}, A}$ é isométrico $a\langle X, d\rangle$.

(2) Para todo $x \in X,\langle X, d\rangle, \mathcal{U}, c_{I, X, x}$ é isométrico $a\langle X, d\rangle$.

Prova. (i) : Como $X$ é não vazio, fixe um $a \in X$. Lembre-se que, para cada $x \in X, c_{I, X, x}$ denota a função constante de valor $a$ de $I$ em $X$ (cf. Comentário 2.2.2). Já que $A$ contém 
qualquer constante em $X$, então $c_{I, X, a} \in A$. Fixe arbitrariamente um $\xi \in A$. Como $A$ é finitamente distal segundo $d$, tem-se que $c_{I, X, a}$ está finitamente distante de $\xi$ segundo d. Fixe um $\delta \in \mathbb{R}^{+}$tal que

$$
\left\{d\left(c_{I, X, a}(i), \xi(i)\right): i \in I\right\} \subseteq[0, \delta] .
$$

É claro que:

$$
\{d(\xi(i), a): i \in I\}=\{d(a, \xi(i)): i \in I\}=\left\{d\left(c_{I, X, a}(i), \xi(i)\right): i \in I\right\} .
$$

Logo, $\{\xi(i): i \in I\} \subseteq B_{d}[a, \delta]$. Como $\langle X, d\rangle$ é próprio, tem-se que $\left\langle B_{d}[a, \delta],\left(\tau_{d}\right)_{B_{d}[a, \delta]}\right\rangle$ é compacto. Como $\left\langle X, \tau_{d}\right\rangle$ é de Hausdorff (cf. Proposição 1.2.10 (iii)), então existe um único $\mathcal{U}$-limite de $\xi$ com respeito a $\tau_{d}$ (cf. Corolário 2.1.14).

(ii)(1) : Em virtude de (i), pode-se considerar a função $\varphi: A \rightarrow X$ que é definida pondo-se, para todo $\xi \in A$,

$$
\varphi(\xi):=\mathcal{U}-\lim _{i \in I}^{\tau_{d}} \xi(i)
$$

Para quaisquer $\xi, \xi^{\prime} \in A$, a família $\left\langle d\left(\xi(i), \xi^{\prime}(i)\right)\right\rangle_{i \in I} \mathcal{U}$-converge para $d\left(\varphi(\xi), \varphi\left(\xi^{\prime}\right)\right)$ com respeito a $\tau_{2}$ (cf. Corolário 2.1.23(i)), o que implica que

$$
d \mathcal{U}, A\left(\xi, \xi^{\prime}\right)=\mathcal{U}-\lim _{i \in I} d\left(\xi(i), \xi^{\prime}(i)\right)=d\left(\varphi(\xi), \varphi\left(\xi^{\prime}\right)\right) .
$$

Então, $\varphi$ preserva distância segundo $d_{\mathcal{U}, A}$ e $d$. Lembre-se que $\left\{c_{I, X, x}: x \in X\right\} \subseteq A$ e note que, para todo $x \in X, \varphi\left(c_{I, X, x}\right)=x$ (cf. Comentário 2.1.5(iv) (2)). Logo, $\varphi$ é sobrejetora. Portanto, como $d$ é uma métrica sobre $X$, conclui-se usando o Teorema 1.2.13 (ii) (2) que $\langle X, d\rangle^{I, \mathcal{U}, A}$ é isométrico a $\langle X, d\rangle$.

(ii) (2) : Segue imediatamente dos itens (i) e (viii) (1) do Comentário 2.2.2 e de (ii) (1).

Uma consequência imediata dos Teoremas 2.2.10 e 1.2.26(vi) é o seguinte

Corolário 2.2.16. Sejam I um conjunto não vazio e $\mathcal{U}$ um ultrafiltro sobre I. Sejam $\left\{\left\langle X_{i}, d_{i}\right\rangle: i \in I\right\}$ uma família de espaços pseudométricos completos tal que o produto cartesiano $\prod_{i \in I} X_{i}$ é não vazio e A um subconjunto de $\prod_{i \in I} X_{i}$ que é $\mathcal{U}$-finitamente distal com respeito a $\left\{d_{i}: i \in I\right\}$. Assumindo-se AC ou, assumindo-se $\mathbf{A C}_{\omega}$ e supondo-se que, para cada $i \in I, d_{i}$ seja uma métrica sobre $X_{i}$, tem-se que:

(i) $\prod_{I, \mathcal{U}, A}\left\langle X_{i}, d_{i}\right\rangle$ é completo.

(ii) Para todo $\xi \in \prod_{i \in I} X_{i}, \prod_{I, \mathcal{U}, \xi}\left\langle X_{i}, d_{i}\right\rangle$ é completo. 
Teorema 2.2.17. Sejam I um conjunto não vazio e $\mathcal{U}$ um ultrafiltro sobre I. Sejam $\left\{\left\langle X_{i}, d_{i}\right\rangle: i \in I\right\}$ e $\left\{\left\langle X_{i}^{\prime}, d_{i}^{\prime}\right\rangle: i \in I\right\}$ famílias de espaços pseudométricos tais que os produtos cartesianos $\prod_{i \in I} X_{i}$ e $\prod_{i \in I} X_{i}^{\prime}$ são não vazios e A um subconjunto de $\prod_{i \in I} X_{i}$ que é finitamente distal com respeito a $\left\{d_{i}: i \in I\right\}$. Seja $\left\{\varphi_{i}: i \in I\right\}$ uma família de funções tais que, para todo $i \in I, \varphi_{i}$ é uma função de $X_{i}$ em $X_{i}^{\prime}$ que preserva distância segundo $d_{i}$ e $d_{i}^{\prime}$. Então:

(i) $\prod_{I, \mathcal{U}, A}\left\langle X_{i}, d_{i}\right\rangle$ é isométrico a $\prod_{I, \mathcal{U},\left(\prod_{i \in I} \varphi_{i}\right)[A]}\left\langle X_{i}^{\prime}, d_{i}^{\prime}\right\rangle$.

(ii) Supondo-se que, para cada $i \in I, \varphi_{i}$ seja uma isometria segundo $d_{i}$ e $d_{i}^{\prime}$ ou, assumindo-se AC e supondo-se que, para cada $i \in I, \varphi_{i}$ seja uma sobrejeção, tem-se que, para todo $\xi \in \prod_{i \in I} X_{i}$
(1) $\left(\prod_{i \in I} \varphi_{i}\right)\left[\ell_{\infty, \xi}\left(I, X_{i}, d_{i}\right)\right]=\ell_{\infty},\left(\prod_{i \in I} \varphi_{i}\right)(\xi)\left(I, X_{i}^{\prime}, d_{i}^{\prime}\right)$
(2) $\prod_{I, \mathcal{U}, \xi}\left\langle X_{i}, d_{i}\right\rangle$ é isométrico a $\prod_{I, \mathcal{U},\left(\prod_{i \in I} \varphi_{i}\right)(\xi)}\left\langle X_{i}^{\prime}, d_{i}^{\prime}\right\rangle$.

Prova. (i) : Considere a função $\varphi: A \rightarrow \prod \quad X_{i}^{\prime}$ que é definida pondo-se, para todo $\xi \in A$,

$$
I, d_{i}^{\prime}, \mathcal{U},\left(\prod_{i \in I} \varphi_{i}\right)[A]
$$

$$
\varphi(\xi):=\mathcal{c}_{0, \mathcal{U}}\left(\prod_{i \in I} \varphi_{i}\right)(\xi),\left(\prod_{i \in I} \varphi_{i}\right)_{[A]}\left(I, d_{i}^{\prime}\right) .
$$

É imediato desta definição que $\varphi$ é sobrejetora. Como, para todo $i \in I, \varphi_{i}$ preserva distância segundo $d_{i}$ e $d_{i}^{\prime}$, tem-se que, para quaisquer $\xi, \xi^{\prime} \in A$,

$$
\begin{aligned}
\left(\bigotimes_{I, \mathcal{U},\left(\prod_{i \in I} \varphi_{i}\right)[A]} d_{i}^{\prime}\right)\left(\varphi(\xi), \varphi\left(\xi^{\prime}\right)\right) & =\left(d_{i}^{\prime}\right)_{I, \mathcal{U}}\left(\prod_{i \in I} \varphi_{i}\right)[A]\left(\left(\prod_{i \in I} \varphi_{i}\right)(\xi),\left(\prod_{i \in I} \varphi_{i}\right)\left(\xi^{\prime}\right)\right)= \\
& \left.=\mathcal{U}-\lim d_{j}^{\prime}\left(\left(\prod_{i \in I} \varphi_{i}\right)(\xi)\right)(j),\left(\left(\prod_{i \in I} \varphi_{i}\right)\left(\xi^{\prime}\right)\right)(j)\right)= \\
& =\mathcal{U}-\lim d_{j}^{\prime}\left(\varphi_{j}(\xi(j)), \varphi_{j}\left(\xi^{\prime}(j)\right)\right)= \\
& =\mathcal{U}-\lim d_{j}\left(\xi(j), \xi^{\prime}(j)\right)=\left(d_{i}\right)_{I, \mathcal{U}, A}\left(\xi, \xi^{\prime}\right) .
\end{aligned}
$$


Então, $\varphi$ preserva distância segundo $\left(d_{i}\right)_{I, \mathcal{U}, A}$ e $\bigotimes \quad d_{i}^{\prime}$. Portanto, já que $I, \mathcal{U},\left(\prod_{i \in I} \varphi_{i}\right)[A]$

$\bigotimes \quad d_{i}^{\prime}$ é uma métrica sobre $\prod X_{i}^{\prime}$, conclui-se usando o Teorema $I, \mathcal{U},\left(\prod_{i \in I} \varphi_{i}\right)[A] \quad I, d^{\prime}, \mathcal{U},\left(\prod_{i \in I} \varphi_{i}\right)[A]$

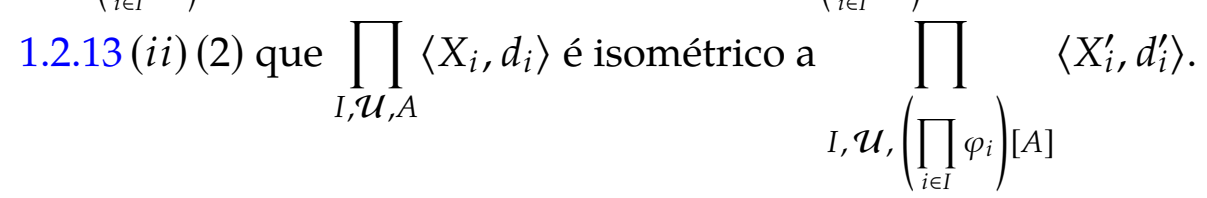

(ii)(1) : Tome um $\eta \in \prod_{i \in I} X_{i}^{\prime}$ qualquer. Suponha que, para cada $i \in I, \varphi_{i}$ seja uma sobrejeção. Sendo assim, para todo $i \in I$, o conjunto $A_{i}:=\left(\varphi_{i}\right)^{-1}[\{\eta(i)\}]$ é não vazio. Considere então a família $\mathcal{A}_{\eta}:=\left\{A_{i}: i \in I\right\}$.

Assuma AC: fixe uma função escolha $\phi$ para $\mathcal{A}_{\eta}$ (i.e., uma função de $\mathcal{A}_{\eta}$ em $\cup \mathcal{A}_{\eta}$ tal que, para todo $i \in I$,

$$
\left.\phi\left(A_{i}\right) \in A_{i}\right)
$$

Considere a função $\xi_{\eta, \phi}: I \rightarrow \bigcup_{i \in I} X_{i}$ que é definida pondo-se, para todo $i \in I$,

$$
\xi_{\eta, \phi}(i):=\phi\left(A_{i}\right)
$$

É claro que $\xi_{\eta, \phi} \in \prod_{i \in I} X_{i}$ e que $\left(\prod_{i \in I} \varphi_{i}\right)\left(\xi_{\eta, \phi}\right)=\eta$.

Suponha que, para cada $i \in I, \varphi_{i}$ seja uma bijeção. Considere a função $\xi_{\eta}: I \rightarrow \bigcup_{i \in I} X_{i}$ que é definida pondo-se, para todo $i \in I$,

$$
\xi_{\eta}(i):=\left(\varphi_{i}\right)^{-1}(\eta(i))
$$

É claro que $\xi_{\eta} \in \prod_{i \in I} X_{i}$ e que $\left(\prod_{i \in I} \varphi_{i}\right)\left(\xi_{\eta}\right)=\eta$.

Fixe arbitrariamente um $\xi \in \prod_{i \in I} X_{i}$. Já é sabido que

$$
\left(\prod_{i \in I} \varphi_{i}\right)\left[\ell_{\infty, \xi}\left(I, d_{i}\right)\right] \subseteq \ell_{\infty,}\left(\prod_{i \in I} \varphi_{i}\right)(\xi)\left(I, d_{i}^{\prime}\right)
$$


(v. Comentário 2.2.2 (ix)). Tome um $\eta \in \ell_{\infty},\left(\prod_{i \in I} \varphi_{i}\right)(\xi) \quad\left(I, d_{i}^{\prime}\right)$ qualquer. Assim, tem-se que $\eta$ está finitamente distante de $\left(\prod_{i \in I} \varphi_{i}\right)(\xi)$ com respeito a $\left\{d_{i}^{\prime}: i \in I\right\}$. Fixe um $\delta \in \mathbb{R}^{+}$tal que

$$
\left\{d_{i}^{\prime}\left(\eta(i),\left(\left(\prod_{i \in I} \varphi_{i}\right)(\xi)\right)(i)\right): i \in I\right\} \subseteq[0, \delta] .
$$

Fixe agora $\operatorname{um} \zeta \in \prod_{i \in I} X_{i}$ tal que $\left(\prod_{i \in I} \varphi_{i}\right)(\zeta)=\eta$. Já que, para todo $i \in I, \varphi_{i}$ preserva distância segundo $d_{i}$ e $d_{i}^{\prime}$, tem-se que:

$$
\begin{aligned}
\left\{d_{i}(\zeta(i), \xi(i)): i \in I\right\} & =\left\{d_{i}^{\prime}\left(\varphi_{i}(\zeta(i)), \varphi_{i}(\xi(i))\right): i \in I\right\}= \\
& \left.=\left\{d_{i}^{\prime}\left(\left(\left(\prod_{i \in I} \varphi_{i}\right)(\zeta)\right)(i), \int\left(\prod_{i \in I} \varphi_{i}\right)(\xi)\right)(i)\right): i \in I\right\}= \\
& =\left\{d_{i}^{\prime}\left(\eta(i),\left(\left(\prod_{i \in I} \varphi_{i}\right)(\xi)\right)(i)\right): i \in I\right\}
\end{aligned}
$$

Logo, $\zeta$ está finitamente distante de $\xi$ com respeito a $\left\{d_{i}: i \in I\right\}$, o que equivale a $\zeta \in \ell_{\infty, \xi}\left(I, d_{i}\right)$. Assim, tem-se que $\eta \in\left(\prod_{i \in I} \varphi_{i}\right)\left[\ell_{\infty, \xi}\left(I, d_{i}\right)\right]$.

(ii) (2) : Segue imediatamente do Comentário 2.2.2 (viii)(1)(1), de (i) e de (ii)(1).

No que segue, são apresentadas definições e comentários referentes a produtos de espaços pseudonormados sobre um mesmo subcorpo de $\mathbb{C}$ e estabelecidos resultados análogos a outros já apresentados nesta seção.

Definição 2.2.18. Sejam $I$ um conjunto não vazio e $\mathcal{U}$ um ultrafiltro sobre $I$. Seja $\mathbb{K}$ um subcorpo de $\mathbb{C}$. Sejam $\left\{\left\langle X_{i},\|\cdot\|_{i}\right\rangle: i \in I\right\}$ uma família de espaços pseudonormados sobre $\mathbb{K}, A \subseteq \prod_{i \in I} X_{i}$ e $\alpha, \beta \in \prod_{i \in I} X_{i}$. Seja $\langle X,\|\cdot\|\rangle$ um espaço pseudonormado sobre $\mathbb{K}$. Diremos que:

(i) $\alpha$ está finitamente distante de $\beta$ com respeito a $\left\{\|\cdot\|_{i}: i \in I\right\}$ se $\alpha$ estiver finitamente distante de $\beta$ com respeito a $\left\{d_{\|\cdot\|_{i}}: i \in I\right\}$.

(ii) A é finitamente distal com respeito a $\left\{\|\cdot\|_{i}: i \in I\right\}$ se $A$ for finitamente distal com respeito a $\left\{d_{\|\cdot\|_{i}}: i \in I\right\}$.

Suponhamos que, para cada $i \in I,\left\langle X_{i},\|\cdot\|_{i}\right\rangle=\langle X,\|\cdot\|\rangle$. Diremos que: 
(iii) $\alpha$ está finitamente distante de $\beta$ segundo $\|\cdot\|$ se $\alpha$ estiver finitamente distante de $\beta$ com respeito a $\left\{\|\cdot\|_{i}: i \in I\right\}$.

(iv) $A$ é finitamente distal segundo $\|\cdot\|$ se $A$ for finitamente distal com respeito a $\left\{\|\cdot\|_{i}: i \in I\right\}$.

Comentário 2.2.19. Dados um conjunto não vazio $I$, um subcorpo $\mathbb{K}$ de $\mathbb{C}$ e uma família $\left\{X_{i}: i \in I\right\}$ de espaços vetoriais sobre $\mathbb{K}$, note que o produto cartesiano $\prod_{i \in I} X_{i}$ é um espaço vetorial sobre $\mathbb{K}$ quando munido da adição e da multiplicação por escalar de $\mathbb{K}$ que são definidas coordenada a coordenada - e o seu vetor nulo é o elemento de $\prod_{i \in I} X_{i}$ denotado por 0 e tal que, para todo $i \in I, 0(i)$ é o vetor nulo de $X_{i}$. Denote por $\mathbb{K}\left(\prod_{i \in I} X_{i}\right)$ tal espaço vetorial. Dada uma família $\left\{\|\cdot\|_{i}: i \in I\right\}$ de funções tais que, para todo $i \in I,\|\cdot\|_{i}$ é uma pseudonorma sobre $X_{i}$, para cada $\xi \in \prod_{i \in I} X_{i}$, o conjunto $\ell_{\infty, \xi}\left(I, X_{i}, d_{\|\cdot\|_{i}}\right)$ será denotado por $\ell_{\infty, \xi}\left(I, X_{i},\|\cdot\|_{i}\right)$ (ou, simplesmente, por $\ell_{\infty, \xi}\left(I,\|\cdot\|_{i}\right)$ ). O conjunto $\ell_{\infty, 0}\left(I, X_{i},\|\cdot\|_{i}\right)$ será denotado por $\ell_{\infty}\left(I, X_{i},\|\cdot\|_{i}\right)$ (ou, simplesmente, por $\left.\ell_{\infty}\left(I,\|\cdot\|_{i}\right)\right)$. Usando o Comentário 2.2.2 $(x)$, tem-se que:

(i) $\ell_{\infty}(I, \mathbb{R},|\cdot|)=\ell_{\infty}(I)$.

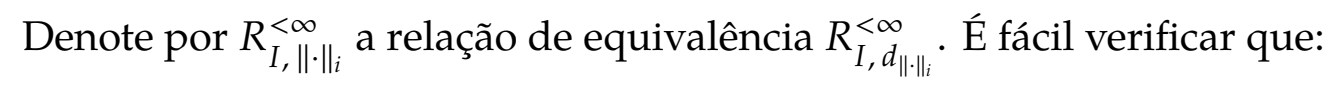

(ii) $R_{I,\|\cdot\|_{i}}^{<\infty}$ é uma relação de congruência sobre $\mathbb{K}\left(\prod_{i \in I} X_{i}\right)$ (i.e., vale a seguinte condição: para quaisquer $\xi, \xi^{\prime}, \zeta, \zeta^{\prime} \in \prod_{i \in I} X_{i}$ e todo $\lambda \in \mathbb{K}$, se $\xi R_{I,\|\cdot\|_{i}}^{<\infty} \xi^{\prime}$ e $\zeta R_{I,\|\cdot\|_{i}}^{<\infty} \zeta^{\prime}$, então $\xi+\zeta R_{I,\|\cdot\|_{i}}^{<\infty} \xi^{\prime}+\zeta^{\prime}$ e $\left.\lambda \xi R_{I,\|\cdot\|_{i}}^{<\infty} \lambda \xi^{\prime}\right)$.

Usando o item (ii), conclui-se que:

(iii) (1) $\ell_{\infty}\left(I, X_{i},\|\cdot\|_{i}\right)$ é um subespaço vetorial de $\mathrm{K}\left(\prod_{i \in I} X_{i}\right)$.

(2) Para todo $\xi \in \prod_{i \in I} X_{i}, \ell_{\infty, \xi}\left(I, X_{i},\|\cdot\|_{i}\right)=\xi+\ell_{\infty}\left(I, X_{i},\|\cdot\|_{i}\right)$.

Agora, dado um subconjunto $A$ de $\prod_{i \in I} X_{i}$ que é finitamente distal com respeito a $\left\{\|\cdot\|_{i}: i \in I\right\}$, para cada $\xi \in A$, o conjunto $c_{0, \mathcal{U}, \xi, A}\left(I, X_{i}, d_{\|\cdot\|_{i}}\right)$ será denotado por 
$\mathcal{c}_{0, \mathcal{U}, \xi, A}\left(I, X_{i},\|\cdot\|_{i}\right)$ (ou, simplesmente, por $\left.c_{0, \mathcal{U}, \xi, A}\left(I,\|\cdot\|_{i}\right)\right)$. Para cada $\xi \in \prod_{i \in I} X_{i}$, sempre que for conveniente, o conjunto $c_{0, \mathcal{U}, \xi, \ell_{\infty, \xi}\left(I, X_{i},\|\cdot\|_{i}\right)}\left(I, X_{i},\|\cdot\|_{i}\right)$ será denotado por $c_{0, \mathcal{U}, \xi}\left(I, X_{i},\|\cdot\|_{i}\right)$ (ou, simplesmente, por $c_{0, \mathcal{U}, \xi}\left(I,\|\cdot\|_{i}\right)$ ). O conjunto $c_{0, \mathcal{U}, 0}\left(I, X_{i},\|\cdot\|_{i}\right)$ será denotado por $\mathcal{c}_{0, \mathcal{U}}\left(I, X_{i},\|\cdot\|_{i}\right)$ (ou, simplesmente, por $\mathcal{c}_{0, \mathcal{U}}\left(I,\|\cdot\|_{i}\right)$ ). Suponha que $A$ seja um subespaço vetorial de $\mathbb{K}\left(\prod_{i \in I} X_{i}\right)$. Denote por ${ }_{\mathbb{K}} A$ o conjunto $A$ munido de sua estrutura de subespaço vetorial de $\mathrm{K}\left(\prod_{i \in I} X_{i}\right)$. É corriqueiro verificar que:

(iv) (1) $\mathcal{c}_{0, \mathcal{U}, 0, A}\left(I, X_{i},\|\cdot\|_{i}\right)$ é um subespaço vetorial de $\mathbb{K} A$.

(2) Para todo $\xi \in A, c_{0, \mathcal{U}, \xi, A}\left(I, X_{i},\|\cdot\|_{i}\right)=\xi+c_{0, \mathcal{U}, 0, A}\left(I, X_{i},\|\cdot\|_{i}\right)$.

Em virtude do item (iv) e do Comentário 2.2.2(viii)(1) (1), tem-se que:

(v) (1) $c_{0, \mathcal{U}}\left(I, X_{i},\|\cdot\|_{i}\right)$ é um subespaço vetorial de $\mathbb{K}_{\infty}\left(I, X_{i},\|\cdot\|_{i}\right)$.

(2) Para todo $\xi \in \ell_{\infty}\left(I, X_{i},\|\cdot\|_{i}\right), c_{0, \mathcal{U}, \xi}\left(I, X_{i},\|\cdot\|_{i}\right)=\xi+c_{0, \mathcal{U}}\left(I, X_{i},\|\cdot\|_{i}\right)$.

Agora, dadas uma família $\left\{X_{i}^{\prime}: i \in I\right\}$ de espaços vetoriais sobre $\mathbb{K}$ e uma família $\left\{\Phi_{i}: i \in I\right\}$ de funções tais que, para todo $i \in I, \Phi_{i}$ é uma transformação linear de $X_{i}$ em $X_{i}^{\prime}$, é fácil ver que:

(1) $\prod_{i \in I} \Phi_{i}$ é uma transformação linear.

(2) $\left(\prod_{i \in I} \Phi_{i}\right)[A]$ é um subespaço vetorial de $\mathbb{K}\left(\prod_{i \in I} X_{i}^{\prime}\right)$.

Em virtude do item (vi) (1) e do Comentário 2.2.2(ix), tem-se que:

(vii) $\left(\prod_{i \in I} \Phi_{i}\right)\left[\ell_{\infty}\left(I, X_{i},\|\cdot\|_{i}\right)\right] \subseteq \ell_{\infty}\left(I, X_{i}^{\prime}, d_{i}^{\prime}\right)$.

Comentário 2.2.20. Dados um conjunto não vazio $I$, um ultrafiltro $\mathcal{U}$ sobre $I$, um subcorpo $\mathbb{K}$ de $\mathbb{C}$, uma família $\left\{\left\langle X_{i},\|\cdot\|_{i}\right\rangle: i \in I\right\}$ de espaços pseudonormados sobre $\mathbb{K}$ e um subespaço vetorial $A$ de $\mathbb{K}\left(\prod_{i \in I} X_{i}\right)$ que é finitamente distal com respeito a $\left\{\|\cdot\|_{i}: i \in I\right\}$, considere as funções $\left(\|\cdot\|_{i}\right)_{I, \infty, A},\left(\|\cdot\|_{i}\right)_{I, \mathcal{U}, A}: A \rightarrow \mathbb{R}$ que são definidas pondo-se, para todo $\xi \in A$,

$$
\begin{aligned}
\left(\|\xi\|_{i}\right)_{I, \infty, A} & :=\left(d_{\|\cdot\|_{i}}\right)_{I, \infty, A}(\xi, 0) \\
& \mathrm{e} \\
\left(\|\xi\|_{i}\right)_{I, \mathcal{U}, A} & :=\left(d_{\|\cdot\|_{i}}\right)_{I, \mathcal{U}, A}(\xi, 0) .
\end{aligned}
$$


Note que:

(i) Para todo $\xi \in A$,

(1) $\left(\|\xi\|_{i, \infty, A}=\sup \left\{\|\xi(i)\|_{i}: i \in I\right\}\right.$.

(2) $\left(\|\xi\|_{i}\right)_{I, \mathcal{U}, A}=\mathcal{U}-\lim _{i \in I}\|\xi(i)\|_{i}$.

Usando o item (i), tem-se que:

(ii) (1) $d_{\left(\|\cdot\|_{i}\right)_{I, \infty, A}}=\left(d_{\|\cdot\|_{i}}\right)_{I, \infty, A}$.

(2) $d_{\left(\|\cdot\|_{i}\right)_{I, \mathcal{U}, A}}=\left(d_{\|\cdot\|_{i}}\right)_{I, \mathcal{U}, A}$.

Com a utilização da homogeneidade não negativa da função supremo para uma família de funções com o mesmo domínio e limitadas superiormente no corpo ordenado $\mathbb{R}$ e com o uso do item (ii), dos Comentários 1.2.28(v)(1), 2.2.3 (ii) (2) e 2.1.5(iv) (2) e do Corolário 2.1.21 (ii), conclui-se que:

(iii) (1) $\left(\|\cdot\|_{i}\right)_{I, \infty, A}$ é uma pseudonorma sobre $A$.

(2) $\left(\|\cdot\|_{i}\right)_{I, \mathcal{U}, A}$ é uma pseudonorma sobre $A$.

Lembre-se que, para cada $j \in I, p_{j}$ está denotando a projeção $p_{I, X_{i}, j}$ (v. Comentário 1.1.23). Note que:

(iv) Para todo $i \in I$,

(1) $p_{i}$ é uma transformação linear.

(2) $p_{i}[A]$ é um subespaço vetorial de $X_{i}$.

Lembre-se que, para cada $i \in I,\left(\|\cdot\|_{i}\right)_{p_{i}[A]}$ denota a pseudonorma induzida por $\|\cdot\|_{i}$ sobre $p_{i}[A]$ (cf. Comentário 1.2.28). Com o uso dos itens (i) (1) e (iii) (1) e do Comentário 1.2.28 (ii) (1), conclui-se que:

(v) Se, para todo $i \in I,\left(\|\cdot\|_{i}\right)_{p_{i}[A]}$ for uma norma sobre $p_{i}[A]$, então $\left(\|\cdot\|_{i}\right)_{I, \infty, A}$ é uma norma sobre $A$.

Com a utilização da homogeneidade não negativa da função supremo para uma família de funções com o mesmo domínio e limitadas superiormente no corpo ordenado $\mathbb{R}$ e com o uso do item (ii) acima, dos itens (v) (2) e (x)(2) (1) do Comentário 1.2.28, dos Comentários 2.2.3 (iv) (2) e 2.1.5 (iv) (2) e do Corolário 2.1.21 (ii), conclui-se que:

(vi) Se, para todo $i \in I,\left(\|\cdot\|_{i}\right)_{p_{i}[A]}$ for uma ultrapseudonorma sobre $p_{i}[A]$, então 
(1) $\left(\|\cdot\|_{i}\right)_{I, \infty, A}$ é uma ultrapseudonorma sobre $A$.

(2) $\left(\|\cdot\|_{i}\right)_{I, \mathcal{U}, A}$ é uma ultrapseudonorma sobre $A$.

Com o uso dos itens (v) e (vi) (1) acima e dos itens (ii) e (iii) (2) do Comentário 1.2.28, conclui-se que:

(vii) Se, para todo $i \in I,\left(\|\cdot\|_{i}\right)_{p_{i}[A]}$ for uma ultranorma sobre $p_{i}[A]$, então $\left(\|\cdot\|_{i}\right)_{I, \infty, A}$ é uma ultranorma sobre $A$.

Sempre que for conveniente, as pseudonormas $\left(\|\cdot\|_{i}\right)_{I, \infty, \ell_{\infty}\left(I, X_{i},\|\cdot\|_{i}\right)}$ e $\left(\|\cdot\|_{i}\right)_{I, \mathcal{U}, \ell_{\infty}\left(I, X_{i},\|\cdot\|_{i}\right)}$ serão denotadas, respectivamente, por $\left(\|\cdot\|_{i}\right)_{I, \infty}$ e por $\left(\|\cdot\|_{i}\right)_{I, \mathcal{U}}$. Dado um espaço pseudonormado $\langle X,\|\cdot\|\rangle$ sobre $\mathbb{K}$, caso tenhamos, para cada $i \in I,\left\langle X_{i},\|\cdot\|_{i}\right\rangle=\langle X,\|\cdot\|\rangle$, denotaremos por $\|\cdot\|_{\infty, A}$ e por $\|\cdot\|_{\mathcal{U}, A}$, respectivamente, as pseudonormas $\left(\|\cdot\|_{i}\right)_{I, \infty, A}$ e $\left(\|\cdot\|_{i}\right)_{I, \mathcal{U}, A} \cdot$

O corolário a seguir é imediato da Proposição 2.2.5 e dos Comentários 2.2.20 (ii) (2) e 1.2.28(v)(1).

Corolário 2.2.21. Sejam I um conjunto não vazio e $\mathcal{U}$ um ultrafiltro sobre I. Seja $\mathbb{K} u m$ subcorpo de $\mathbb{C}$. Sejam $\left\{\left\langle X_{i},\|\cdot\|_{i}\right\rangle: i \in I\right\}$ uma família de espaços pseudonormados sobre $\mathbb{K} e$ A um subespaço vetorial de $\mathbb{K}\left(\prod_{i \in I} X_{i}\right)$ que é finitamente distal com respeito a $\left\{\|\cdot\|_{i}: i \in I\right\}$. Então:

(i) Para todo $\xi \in A$,

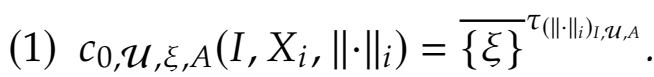

(2) $c_{0, \mathcal{U}, \xi, A}\left(I, X_{i},\|\cdot\|_{i}\right)$ é um fechado em $\left\langle A, \tau_{\left(\|\cdot\|_{i}\right)_{I, \mathcal{U}, A}}\right\rangle$.

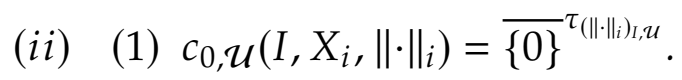

(2) $c_{0, \mathcal{U}}\left(I, X_{i},\|\cdot\|_{i}\right)$ é um fechado em $\left\langle\ell_{\infty}\left(I, X_{i},\|\cdot\|_{i}\right), \tau_{\left(\|\cdot\|_{i}\right)_{I, \mathcal{U}}}\right\rangle$.

Definição 2.2.22. Sejam $I$ um conjunto não vazio e $\mathcal{U}$ um ultrafiltro sobre $I$. Seja $\mathbb{K}$ um subcorpo de $\mathbb{C}$. Sejam $\left\{\left\langle X_{i},\|\cdot\|_{i}\right\rangle: i \in I\right\}$ uma família de espaços pseudonormados sobre $\mathbb{K}$ e $A \subseteq \prod_{i \in I} X_{i}$. Seja $\langle X,\|\cdot\|\rangle$ um espaço pseudonormado sobre $\mathbb{K}$. Diremos que:

(i) $A$ é sup-finitamente distal com respeito a $\left\{\|\cdot\|_{i}: i \in I\right\}$ se $A$ for sup-finitamente distal com respeito a $\left\{d_{\|\cdot\|_{i}}: i \in I\right\}$. 
(ii) $A$ é $\mathcal{U}$-finitamente distal com respeito a $\left\{\|\cdot\|_{i}: i \in I\right\}$ se $A$ for $\mathcal{U}$-finitamente distal com respeito a $\left\{d_{\|\cdot\|_{i}}: i \in I\right\}$.

(iii) $A$ é $\mathcal{U}$-sup-finitamente distal com respeito a $\left\{\|\cdot\|_{i}: i \in I\right\}$ se $A$ for $\mathcal{U}$-supfinitamente distal com respeito a $\left\{d_{\|\cdot\|_{i}}: i \in I\right\}$.

Suponhamos que, para cada $i \in I,\left\langle X_{i},\|\cdot\|_{i}\right\rangle=\langle X,\|\cdot\|\rangle$. Diremos que:

(iv) $A$ é sup-finitamente distal segundo $\|\cdot\|$ se $A$ for sup-finitamente distal com respeito a $\left\{\|\cdot\|_{i}: i \in I\right\}$.

(v) A é $\mathcal{U}$-finitamente distal segundo $\|\cdot\|$ se $A$ for $\mathcal{U}$-finitamente distal com respeito a $\left\{\|\cdot\|_{i}: i \in I\right\}$.

(vi) $A$ é $\mathcal{U}$-sup-finitamente distal segundo $\|\cdot\|$ se $A$ for $\mathcal{U}$-sup-finitamente distal com respeito a $\left\{\|\cdot\|_{i}: i \in I\right\}$.

Uma consequência imediata da Proposição 2.2.8 e do Comentário 1.2.28 (v) (1) é o seguinte

Corolário 2.2.23. Sejam I um conjunto não vazio e $\mathcal{U}$ um ultrafiltro sobre I. Seja $\mathbb{K} u m$ subcorpo de $\mathbb{C}$. Sejam $\left\{\left\langle X_{i},\|\cdot\|_{i}\right\rangle: i \in I\right\}$ uma família de espaços pseudonormados sobre $\mathbb{K} e$ $A \subseteq \prod_{i \in I} X_{i}$. Então:

(i) Se A for $\mathcal{U}$-sup-finitamente distal com respeito a $\left\{\|\cdot\|_{i}: i \in I\right\}$, então

(1) A é sup-finitamente distal com respeito a $\left\{\|\cdot\|_{i}: i \in I\right\}$.

(2) A é $\mathcal{U}$-finitamente distal com respeito a $\left\{\|\cdot\|_{i}: i \in I\right\}$.

(ii) Para todo $\xi \in \prod_{i \in I} X_{i}$

(1) $\{\xi\}$ é $\mathcal{U}$-sup-finitamente distal com respeito a $\left\{\|\cdot\|_{i}: i \in I\right\}$.

(2) $\ell_{\infty, \xi}\left(I, X_{i},\|\cdot\|_{i}\right)$ é $\mathcal{U}$-sup-finitamente distal com respeito a $\left\{\|\cdot\|_{i}: i \in I\right\}$.

O próximo corolário é imediato do Teorema 2.2.9, do Comentário 2.2.20 (ii) (1) e do Comentário 1.2.28 (v) (1) ou do Comentário 1.2.28(vi). 
Corolário 2.2.24. Seja I um conjunto não vazio. Seja $\mathbb{K}$ um subcorpo de $\mathbb{C}$. Sejam $\left\{\left\langle X_{i},\|\cdot\|_{i}\right\rangle: i \in I\right\}$ uma família de espaços pseudonormados sobre $\mathbb{K}$ que são completos e $A$ um subespaço vetorial de $\mathbb{K}\left(\prod_{i \in I} X_{i}\right)$ que é sup-finitamente distal com respeito a $\left\{\|\cdot\|_{i}: i \in I\right\}$. Assumindo-se AC ou, supondo-se que, para cada $i \in I,\|\cdot\|_{i}$ seja uma norma sobre $X_{i}$, tem-se que:

(i) $\left(\|\cdot\|_{i}\right)_{I, \infty, A}$ é completa.

(ii) $\left(\|\cdot\|_{i}\right)_{I, \infty}$ é completa.

O corolário a seguir é imediato do Teorema 2.2.10, do Comentário 2.2.20 (ii) (2) e do Comentário 1.2.28 (v) (1) ou do Comentário 1.2.28 (vi).

Corolário 2.2.25. Sejam I um conjunto não vazio e $\mathcal{U}$ um ultrafiltro sobre I. Seja $\mathbb{K} u m$ subcorpo de $\mathbb{C}$. Sejam $\left\{\left\langle X_{i},\|\cdot\|_{i}\right\rangle: i \in I\right\}$ uma família de espaços pseudonormados sobre $\mathbb{K}$ que são completos e A um subespaço vetorial de $\mathbb{K}\left(\prod_{i \in I} X_{i}\right)$ que é $\mathcal{U}$-finitamente distal com respeito $a\left\{\|\cdot\|_{i}: i \in I\right\}$. Assumindo-se AC ou, supondo-se que, para cada $i \in I,\|\cdot\|_{i}$ seja uma norma sobre $X_{i}$, tem-se que:

(i) $\left(\|\cdot\|_{i}\right)_{I, \mathcal{U}, A}$ é completa.

(ii) $\left(\|\cdot\|_{i}\right)_{I, \mathcal{u} \text { é completa. }}$

Em virtude do Comentário 1.2.30, o corolário a seguir é imediato do Teorema 2.2.11 e dos Comentários 2.2.20 (ii) e 1.2.28 (v) (1).

Corolário 2.2.26. Sejam I um conjunto não vazio e $\mathcal{U}$ um ultrafiltro sobre I. Seja $\mathbb{K} u m$ subcorpo de $\mathbb{C}$. Sejam $\left\{\left\langle X_{i},\|\cdot\|_{i}\right\rangle: i \in I\right\}$ uma família de espaços pseudonormados sobre $\mathbb{K}$ e A um subespaço vetorial de $\mathbb{K}\left(\prod_{i \in I} X_{i}\right)$ queé $\mathcal{U}$-sup-finitamente distal com respeito a $\left\{\|\cdot\|_{i}: i \in I\right\}$. Então:

(i) (1) $\left(\|\cdot\|_{i}\right)_{I, \mathcal{U}, A}=\left(\left(\|\cdot\|_{i}\right)_{I, \infty, A}\right)^{\mathcal{C}_{0, \mathcal{U}, 0, A}\left(I, X_{i},\|\cdot\|_{i}\right)}$.

(2) $\left(\|\cdot\|_{i}\right)_{I, \mathcal{U}}=\left(\left(\|\cdot\|_{i}\right)_{I, \infty}\right)^{c_{0, \mathcal{U}}\left(I, X_{i},\|\cdot\|_{i}\right)}$.

(ii) (1) $c_{0, \mathcal{U}, 0, A}\left(I, X_{i},\|\cdot\|_{i}\right)$ é um fechado em $\left\langle A, \tau_{\left(\|\cdot\|_{i}\right)_{I, \infty, A}}\right\rangle$.

(2) $c_{0, \mathcal{U}}\left(I, X_{i},\|\cdot\|_{i}\right)$ é um fechado em $\left\langle\ell_{\infty}\left(I, X_{i},\|\cdot\|_{i}\right), \tau_{\left(\|\cdot\|_{i}\right)_{I, \infty}}\right\rangle$. 
A definição a seguir é uma generalização da definição clássica de ultraproduto de espaços normados e de Banach reais ou complexos, que se encontra, por exemplo, em [DCK1972, p. 316], [S1978, p. 232], [Hei1980, p. 74-75] e [Avi 2013, p. 197-198].

Definição 2.2.27. Sejam $I$ um conjunto não vazio e $\mathcal{U}$ um ultrafiltro sobre $I$. Seja $\mathbb{K}$ um subcorpo de $\mathbb{C}$. Sejam $\left\{\left\langle X_{i},\|\cdot\|_{i}\right\rangle: i \in I\right\}$ uma família de espaços pseudonormados sobre $\mathbb{K}$ e $A$ um subespaço vetorial de $\mathbb{K}\left(\prod_{i \in I} X_{i}\right)$ que é $\mathcal{U}$-sup-finitamente distal com respeito a $\left\{\|\cdot\|_{i}: i \in I\right\}$. Seja $\langle X,\|\cdot\|\rangle$ um espaço pseudonormado sobre $\mathbb{K}$. Denotaremos por $\prod_{I,\|\cdot\|_{i}, \mathcal{U}, A} X_{i}$ o conjunto $A / c_{0, \mathcal{U}, 0, A}\left(I, X_{i},\|\cdot\|_{i}\right)$ e por $\bigotimes_{I, \mathcal{U}, A}\|\cdot\|_{i}$ a norma $\left(\|\cdot\|_{i}\right)_{I, \infty, A} / \mathcal{c}_{0, \mathcal{U}, 0, A}\left(I, X_{i},\|\cdot\|_{i}\right)$. O espaço normado $\left\langle A,\left(\|\cdot\|_{i}\right)_{I, \infty, A}\right\rangle / \mathcal{c}_{0, \mathcal{U}, 0, A}\left(I, X_{i},\|\cdot\|_{i}\right)$ será denotado por $\prod_{I, \mathcal{U}, A}\left\langle X_{i},\|\cdot\|_{i}\right\rangle$. Diremos que:

(i) $\prod_{I, \mathcal{U}, A}\left\langle X_{i},\|\cdot\|_{i}\right\rangle$ é o ultraproduto (normado) de $\left\{\left\langle X_{i},\|\cdot\|_{i}\right\rangle: i \in I\right\}$ com respeito a $\mathcal{U}$ e ancorado em $A$.

Denotaremos por $\prod_{I,\|\cdot\|_{i}, \mathcal{U}} X_{i}$ o conjunto $\prod_{I,\|\cdot\|_{i}, \mathcal{U}, \ell_{\infty}\left(I, X_{i},\|\cdot\|_{i}\right)} X_{i}$ e por $\bigotimes_{I, \mathcal{U}}\|\cdot\|_{i}$ a norma $I, \mathcal{U}, \ell_{\infty}\left(I, X_{i},\|\cdot\|_{i}\right)$ $\|\cdot\|_{i}$. O espaço normado $\prod_{I}\left\langle X_{i},\|\cdot\|_{i}\right\rangle$ será denotado por $\prod_{I, \mathcal{U}}\left\langle X_{i},\|\cdot\|_{i}\right\rangle$. Diremos que: $I, \mathcal{U}, \ell_{\infty}\left(I, X_{i},\|\cdot\|_{i}\right)$

(ii) $\prod_{I, \mathcal{U}}\left\langle X_{i},\|\cdot\|_{i}\right\rangle$ é o ultraproduto (normado) de $\left\{\left\langle X_{i},\|\cdot\|_{i}\right\rangle: i \in I\right\}$ com respeito a $\mathcal{U}$. Suponhamos que, para cada $i \in I,\left\langle X_{i},\|\cdot\|_{i}\right\rangle=\langle X,\|\cdot\|\rangle$. Denotaremos por $X^{I,\|\cdot\|, \mathcal{U}, A}$ (ou, simplesmente, por $X^{\|\cdot\|, \mathcal{U}, A}$ ) o conjunto $\prod_{I,\|\cdot\|_{i}, \mathcal{U}, A} X_{i}$ e por $\|\cdot\|^{I, \mathcal{U}, A}$ (ou, simplesmente, por $\left.\|\cdot\|^{\mathcal{U}, A}\right)$ a norma $\bigotimes_{I, \mathcal{U}, A}\|\cdot\|_{i}$. O espaço normado $\prod_{I, \mathcal{U}, A}\left\langle X_{i},\|\cdot\|_{i}\right\rangle$ será denotado por $\langle X,\|\cdot\|\rangle^{I, \mathcal{U}, A}$ (ou, simplesmente, por $\langle X,\|\cdot\|\rangle^{\mathcal{U}, A}$ ). Diremos que:

(iii) $\langle X,\|\cdot\|\rangle^{I, \mathcal{U}, A}$ é a ultrapotência (normada) de $\langle X,\|\cdot\|\rangle$ com respeito a $\mathcal{U}$ e ancorada em $A$.

Denotaremos por $X^{I,\|\cdot\|, \mathcal{U}}$ (ou, simplesmente, por $X^{\|\cdot\|, \mathcal{U}}$ ) o conjunto $X^{I,\|\cdot\|, \mathcal{U}, \ell_{\infty}\left(I, X_{i},\|\cdot\|_{i}\right)}$ e por $\|\cdot\|^{I, \mathcal{U}}$ (ou, simplesmente, por $\|\cdot\|^{\mathcal{U}}$ ) a norma $\|\cdot\|^{I, \mathcal{U}, \ell_{\infty}\left(I, X_{i},\|\cdot\|_{i}\right)}$. O espaço normado 
$\langle X,\|\cdot\|\rangle^{I, \mathcal{U}, \ell_{\infty}(I, X,\|\cdot\|)}$ será denotado por $\langle X,\|\cdot\|\rangle^{I, \mathcal{U}}$ (ou, simplesmente, por $\langle X,\|\cdot\|\rangle^{\mathcal{U}}$ ). Diremos que:

(iv) $\langle X,\|\cdot\|\rangle^{I, \mathcal{U}}$ é a ultrapotência de $\langle X,\|\cdot\|\rangle$ com respeito a $\mathcal{U}$.

Com o uso do Corolário 2.2.26 (i) (1) e dos Comentários 2.2.19 (iv) (2) e 2.2.20 (ii) (2), conclui-se facilmente o corolário a seguir, que é uma generalização nossa, em termos de métricas induzidas por uma norma, da igualdade que define ou caracteriza a norma de um ultraproduto de espaços normados e de Banach, que pode ser vista, por exemplo, em [DCK1972, p. 316], [Hei1980, p. 75] e [Avi ${ }^{+} 2013$, p. 198].

Corolário 2.2.28. Sejam I um conjunto não vazio e $\mathcal{U}$ um ultrafiltro sobre I. Seja $\mathbb{K} u m$ subcorpo de $\mathbb{C}$. Sejam $\left\{\left\langle X_{i},\|\cdot\|_{i}\right\rangle: i \in I\right\}$ uma família de espaços pseudonormados sobre $\mathbb{K}$ e A um subespaço vetorial de $\mathbb{K}\left(\prod_{i \in I} X_{i}\right)$ queé $\mathcal{U}$-sup-finitamente distal com respeito a $\left\{\|\cdot\|_{i}: i \in I\right\}$. Então:

(i) $d \bigotimes_{I, \mathcal{U}, A}\|\cdot\|_{i}=\bigotimes_{I, \mathcal{U}, A} d_{\|\cdot\|_{i}}$

(ii) $d_{I, \mathcal{U}}\|\cdot\|_{i}=\bigotimes_{I, \mathcal{U}, 0} d_{\|\cdot\|_{i}}$

Com o uso do Teorema 1.2.26 (vi), dos Corolários 2.2.23 (i) (2), 2.2.25 e 2.2.28 e do Comentário 2.2.20 (ii)(2), é fácil concluir o seguinte

Corolário 2.2.29. Sejam I um conjunto não vazio e $\mathcal{U}$ um ultrafiltro sobre I. Seja $\mathbb{K} u m$ subcorpo de $\mathbb{C}$. Sejam $\left\{\left\langle X_{i},\|\cdot\|_{i}\right\rangle: i \in I\right\}$ uma família de espaços pseudonormados sobre $\mathbb{K}$ que são completos e A um subespaço vetorial de $\mathbb{K}\left(\prod_{i \in I} X_{i}\right)$ que é $\mathcal{U}$-sup-finitamente distal com respeito a $\left\{\|\cdot\|_{i}: i \in I\right\}$. Assumindo-se $\mathbf{A C}$ ou, assumindo-se $\mathbf{A C}_{\omega}$ e supondo-se que, para cada $i \in I,\|\cdot\|_{i}$ seja uma norma sobre $X_{i}$, tem-se que:

(i) $\prod_{I, \mathcal{U}, A}\left\langle X_{i},\|\cdot\|_{i}\right\rangle$ é de Banach.

(ii) $\prod_{I, \mathcal{U}}\left\langle X_{i},\|\cdot\|_{i}\right\rangle$ é de Banach.

Encerraremos este capítulo com uma prova do teorema a seguir, que é uma versão do Teorema 2.2.17 para espaços pseudonormados sobre subcorpos quaisquer de $\mathbb{C}$. 
Teorema 2.2.30. Sejam I um conjunto não vazio e $\mathcal{U}$ um ultrafiltro sobre I. Seja $\mathbb{K}$ um subcorpo de C. Sejam $\left\{\left\langle X_{i},\|\cdot\|_{i}\right\rangle: i \in I\right\}$ e $\left\{\left\langle X_{i}^{\prime},\|\cdot\|_{i}^{\prime}\right\rangle: i \in I\right\}$ famílias de espaços pseudonormados sobre $\mathbb{K}$ e A um subespaço vetorial de $\mathbb{K}\left(\prod_{i \in I} X_{i}\right)$ que é $\mathcal{U}$-sup-finitamente distal com respeito a $\left\{\|\cdot\|_{i}: i \in I\right\}$. Seja $\left\{\Phi_{i}: i \in I\right\}$ uma família de funções tais que, para todo $i \in I, \Phi_{i}$ é uma transformação linear de $X_{i}$ em $X_{i}^{\prime}$ que preserva comprimento segundo $\|\cdot\|_{i}$ e $\|\cdot\|_{i}^{\prime}$ e $\left(\prod_{i \in I} \Phi_{i}\right)[A]$ é $\mathcal{U}$-sup-finitamente distal com respeito a $\left\{\|\cdot\|_{i}^{\prime}: i \in I\right\}$. Então:

(i) $\prod_{I, \mathcal{U}, A}\left\langle X_{i},\|\cdot\|_{i}\right\rangle$ é isometricamente isomorfo a $\prod_{I, \mathcal{U},\left(\prod_{i \in I} \Phi_{i}\right)[A]}\left\langle X_{i}^{\prime},\|\cdot\|_{i}^{\prime}\right\rangle$.

(ii) Supondo-se que, para cada $i \in I, \Phi_{i}$ seja um isomorfismo isométrico segundo $\|\cdot\|_{i}$ e $\|\cdot\|_{i}^{\prime}$ ou, assumindo-se AC e supondo-se que, para cada $i \in I, \Phi_{i}$ seja um epimorfismo linear, tem-se que:
(1) $\left(\prod_{i \in I} \Phi_{i}\right)\left[\ell_{\infty}\left(I, X_{i},\|\cdot\|_{i}\right)\right]=\ell_{\infty}\left(I, X_{i}^{\prime}, d_{i}^{\prime}\right)$.
(2) $\prod_{I, \mathcal{U}}\left\langle X_{i},\|\cdot\|_{i}\right\rangle$ é isometricamente isomorfo a $\prod_{I, \mathcal{U}}\left\langle X_{i}^{\prime},\|\cdot\|_{i}^{\prime}\right\rangle$.

Prova. (i) : Considere a função $\Phi: A \rightarrow \prod \quad X_{i}^{\prime}$ que é definida pondo-se, para todo $\xi \in A$,

$$
I,\|\cdot\|_{i}^{\prime}, \mathcal{U},\left(\prod_{i \in I} \Phi_{i}\right)[A]
$$

$$
\Phi(\xi):=\left(\prod_{i \in I} \Phi_{i}\right)(\xi)+\mathcal{c}_{0, \mathcal{U}, 0,}\left(\prod_{i \in I} \Phi_{i}\right)[A]{ }^{\left(I,\|\cdot\|_{i}^{\prime}\right)}
$$

É imediato desta definição que $\Phi$ é sobrejetora. Como $\prod_{i \in I} \Phi_{i}$ é uma transformação linear (cf. Comentário 2.2.19 (vi) (1)), é imediato também desta definição que $\Phi$ é uma transformação linear. Já que

$$
\begin{aligned}
\left(\|\cdot\|_{i}\right)_{I, \mathcal{U}, A} & =\left(\left(\|\cdot\|_{i}\right)_{I, \infty, A}\right)^{\mathcal{C}_{0}, \mathcal{U}, 0, A}\left(I,\|\cdot\|_{i}\right) \\
\mathrm{e} & \\
\left(\|\cdot\|_{i}^{\prime}\right)_{I, \mathcal{U},}\left(\prod_{i \in I} \Phi_{i}\right)_{[A]} & =\left(\left(\|\cdot\|_{i}^{\prime}\right)_{I, \infty,}\left(\prod_{i \in I} \Phi_{i}\right)_{[A]}\right)^{\mathcal{C}_{0, \mathcal{U}, 0,}}\left(\prod_{i \in I} \Phi_{i}\right)^{(A]}\left(I,\|\cdot\|_{i}^{\prime}\right)
\end{aligned}
$$


(cf. Corolário 2.2.26(i)(1)) e, para todo $i \in I, \Phi_{i}$ preserva comprimento segundo $\|\cdot\|_{i}$ e $\|\cdot\|_{i}^{\prime}$, tem-se que, para todo $\xi \in A$,

$$
\begin{aligned}
\bigotimes_{I, \mathcal{U},\left(\prod_{i \in I} \Phi_{i}\right)[A]}\|\Phi(\xi)\|_{i}^{\prime} & \left.=\left(\|\|\left(\prod_{i \in I} \Phi_{i}\right)(\xi) \|_{i}^{\prime}\right)_{I, \infty,\left(\prod_{i \in I} \Phi_{i}\right)[A]}\right)^{c_{0, \mathcal{U}, 0,}\left(\prod_{i \in I} \Phi_{i}\right)[A]}{ }^{\left(I,\|\cdot\|_{i}^{\prime}\right)}= \\
& \left.=\left(\left\|\left(\prod_{i \in I} \Phi_{i}\right)(\xi)\right\|_{i}^{\prime}\right)_{I, \mathcal{U},\left(\prod_{i \in I} \Phi_{i}\right){ }_{[A]}}=\mathcal{U}-\lim \|\left(\| \prod_{i \in I} \Phi_{i}\right)(\xi)\right)(j) \|_{j}^{\prime}= \\
& =\mathcal{U}-\lim \left\|\Phi_{j}(\xi(j))\right\|_{j}^{\prime}=\mathcal{U}-\lim \|\xi(j)\|_{j}=\left(\|\xi\|_{i}\right)_{I, \mathcal{U}, A}= \\
& =\left(\left(\|\xi\|_{i}\right)_{I, \infty, A}\right)^{c_{0, \mathcal{U}, 0, A}\left(I,\|\cdot\|_{i}\right)} .
\end{aligned}
$$

Então, $\Phi$ preserva comprimento segundo $\left(\left(\|\cdot\|_{i}\right)_{I, \infty, A}\right)^{c_{0, \mathcal{U}, 0, A}\left(I,\|\cdot\|_{i}\right)}$ e $\bigotimes \quad\|\cdot\|_{i}^{\prime}$.

$$
I, \mathcal{U},\left(\prod_{i \in I} \Phi_{i}\right)[A]
$$

Portanto, como $c_{0, \mathcal{U}, 0, A}\left(I, X_{i},\|\cdot\|_{i}\right)$ é um fechado em $\left\langle A, \tau_{\left(\|\cdot\|_{i}\right)_{I, \infty, A}}\right\rangle$ e $\bigotimes \quad\|\cdot\|_{i}^{\prime}$ é

$$
I, \mathcal{U},\left(\prod_{i \in I} \Phi_{i}\right)[A]
$$

uma norma sobre $\prod \quad X_{i}^{\prime}$, conclui-se usando o Teorema 1.2.31 (ii) (2) que $I,\|\cdot\|_{i}, \mathcal{U},\left(\prod_{i \in I} \Phi_{i}\right)[A]$

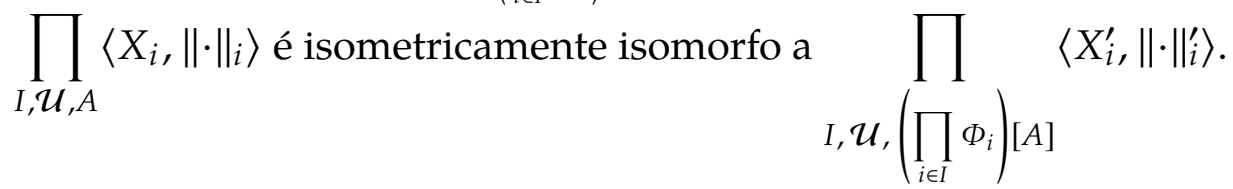

(ii) : Segue imediatamente dos itens (iii) (1) e (vi) (1) do Comentário 2.2.19, do Corolário 2.2.23 (ii), do Teorema 2.2.17(ii) (1) e de (i). 


\section{CAPÍTULO 3}

\section{Ultrassomas topológicas}

Neste capítulo, é apresentada, em ZF + UT, uma introdução detalhada à teoria das ultrassomas de espaços topológicos de Tychonoff. Além disso, é demonstrada, em ZFC, uma generalização da Proposição 2 de [Gur1988] no contexto dos espaços topológicos de Tychonoff.

\subsection{Ultrassomas de espaços topológicos de Tychonoff}

Nesta seção, é feita uma introdução à teoria dos espaços de Stone sobre conjuntos não vazios e à teoria da compactificação de Stone-Čech para estabelecer, em ZF + UT, a definição de ultrassoma de espaços topológicos de Tychonoff.

Definição 3.1.1. Sejam $\langle X, \tau\rangle$ e $\left\langle X^{\prime}, \tau^{\prime}\right\rangle$ espaços topológicos de Tychonoff e $\gamma: X \rightarrow X^{\prime}$ uma função. Considere as seguintes condições:

(a) $\left\langle X^{\prime}, \tau^{\prime}\right\rangle$ é compacto.

(b) $\gamma$ é uma imersão topológica com respeito a $\tau$ e $\tau^{\prime}$.

(c) O conjunto im $(\gamma)$ é um denso em $\left\langle X^{\prime}, \tau^{\prime}\right\rangle$.

(d) Para todo espaço topológico $\langle Z, \sigma\rangle$ compacto e de Tychonoff e toda função $\xi \in C(X, \tau, Z, \sigma)$, existe uma função $\zeta \in C\left(X^{\prime}, \tau^{\prime}, Z, \sigma\right)$ tal que

$$
\zeta \circ \gamma=\xi .
$$


Diremos que:

(i) $\left\langle X^{\prime}, \tau^{\prime}\right\rangle$ é um quase compactificado de $\langle X, \tau\rangle$ segundo $\gamma$ se valerem as condições (a) e $(b)$. Se $\left\langle X^{\prime}, \tau^{\prime}\right\rangle$ for um quase compactificado de $\langle X, \tau\rangle$ segundo $\gamma$, diremos que o terno $\left\langle X^{\prime}, \tau^{\prime}, \gamma\right\rangle$ é uma quase compactificação de $\langle X, \tau\rangle$.

(ii) $\left\langle X^{\prime}, \tau^{\prime}\right\rangle$ é um compactificado de $\langle X, \tau\rangle$ segundo $\gamma$ se $\left\langle X^{\prime}, \tau^{\prime}\right\rangle$ for um quase compactificado de $\langle X, \tau\rangle$ segundo $\gamma$ e valer a condição $(c)$. Se $\left\langle X^{\prime}, \tau^{\prime}\right\rangle$ for um compactificado de $\langle X, \tau\rangle$ segundo $\gamma$, diremos que o terno $\left\langle X^{\prime}, \tau^{\prime}, \gamma\right\rangle$ é uma compactificação de $\langle X, \tau\rangle$.

(iii) $\left\langle X^{\prime}, \tau^{\prime}\right\rangle$ é um compactificado maximal de $\langle X, \tau\rangle$ segundo $\gamma$ se $\left\langle X^{\prime}, \tau^{\prime}\right\rangle$ for um compactificado de $\langle X, \tau\rangle$ segundo $\gamma$ e valer a condição $(d)$. Se $\left\langle X^{\prime}, \tau^{\prime}\right\rangle$ for um compactificado maximal de $\langle X, \tau\rangle$ segundo $\gamma$, diremos que o terno $\left\langle X^{\prime}, \tau^{\prime}, \gamma\right\rangle$ é uma compactificação maximal de $\langle X, \tau\rangle$.

Comentário 3.1.2. Dado um espaço topológico $\langle X, \tau\rangle$ de Tychonoff, note que:

(i) Se $\langle X, \tau\rangle$ for compacto, então $\left\langle X, \tau, \mathrm{id}_{X}\right\rangle$ é uma compactificação maximal de $\langle X, \tau\rangle$.

Dados um espaço topológico $\left\langle X^{\prime}, \tau^{\prime}\right\rangle$ de Tychonoff e uma função $\gamma: X \rightarrow X^{\prime}$, denote por $\bar{\gamma} X$ o conjunto $\overline{\operatorname{im}(\gamma)} \tau^{\prime}$. Denote por $\bar{\gamma} \tau$ e por $\bar{\gamma}$, respectivamente, a topologia $\left(\tau^{\prime}\right)_{\bar{\gamma} X}$ e a correstrição $\gamma \downarrow \bar{\gamma} X$. Afirmamos que:

(ii) Se $\left\langle X^{\prime}, \tau^{\prime}, \gamma\right\rangle$ for uma quase compactificação de $\langle X, \tau\rangle$, então $\langle\bar{\gamma} X, \bar{\gamma} \tau, \bar{\gamma}\rangle$ é uma compactificação de $\langle X, \tau\rangle$.

De fato: lembre-se que $\operatorname{im}(\bar{\gamma})=\operatorname{im}(\varphi)$ (v. Comentário 1.1.12(ii)(2) (1)). Assim, tem-se que:

$$
\bar{\gamma} \lim (\bar{\gamma})=\gamma \lim (\gamma) \text { e }(\bar{\gamma} \tau)_{\operatorname{im}(\bar{\gamma})}=\left(\tau^{\prime}\right)_{\operatorname{im}(\gamma)}
$$

(cf. Comentário 1.1.12(vi)(1)). Supondo-se que $\gamma$ seja uma imersão topológica com respeito a $\tau$ e $\tau^{\prime}$, tem-se então que $\bar{\gamma}$ é uma imersão topológica com respeito a $\tau$ e $\bar{\gamma} \tau$. Além disso, tem-se que:

$$
\overline{\operatorname{im}(\bar{\gamma})}^{\bar{\gamma} \tau}=\overline{\operatorname{im}(\gamma)}^{\bar{\gamma} \tau}=\overline{\operatorname{im}(\gamma)}^{\tau^{\prime}} \cap \bar{\gamma} X=\bar{\gamma} X
$$

(v. Comentário 1.1.12(vii) (2)). Já que $\left\langle X^{\prime}, \tau^{\prime}\right\rangle$ é de Tychonoff, segue que $\langle\bar{\gamma} X, \bar{\gamma} \tau\rangle$ é de Tychonoff (cf. Proposição 1.1.16(v) (2)). Suponha que $\left\langle X^{\prime}, \tau^{\prime}\right\rangle$ seja compacto. Como $\bar{\gamma} X$ 
é um fechado em $\left\langle X^{\prime}, \tau^{\prime}\right\rangle$, então $\langle\bar{\gamma} X, \bar{\gamma} \tau\rangle$ é compacto (cf. Proposição 1.1.13(i)).

Suponha que $\left\langle X^{\prime}, \tau^{\prime}, \gamma\right\rangle$ seja uma compactificação maximal de $\langle X, \tau\rangle$. Com o uso dos itens $(v)(1)$ e (iv)(1) da Proposição 1.1.16 e da Proposição 1.1.21 (ii), é fácil verificar que:

(iii) Para todo espaço topológico $\langle Z, \sigma\rangle$ compacto e de Tychonoff e toda função $\xi \in C(X, \tau, Z, \sigma)$, existe uma única função $\zeta \in C\left(X^{\prime}, \tau^{\prime}, Z, \sigma\right)$ tal que

$$
\zeta \circ \gamma=\xi
$$

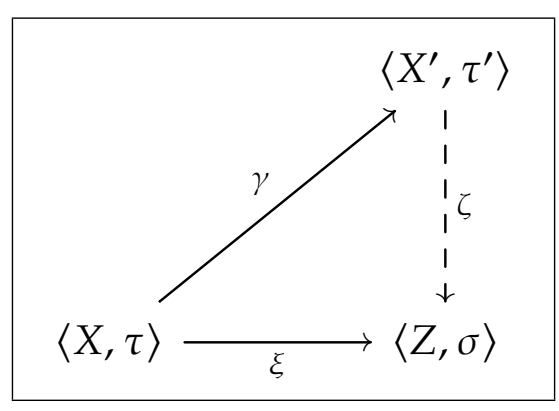

Diagrama iii.1

Dados um espaço topológico $\left\langle X^{\prime \prime}, \tau^{\prime \prime}\right\rangle$ de Tychonoff e uma função $\gamma^{\prime}: X \rightarrow X^{\prime \prime}$, suponha que $\left\langle X^{\prime \prime}, \tau^{\prime \prime}, \gamma^{\prime}\right\rangle$ seja uma compactificação maximal de $\langle X, \tau\rangle$. Afirmamos que:

(iv) Existe um único homeomorfismo $\zeta: X^{\prime} \rightarrow X^{\prime \prime}$ com respeito a $\tau^{\prime}$ e $\tau^{\prime \prime}$ tal que

$$
\zeta \circ \gamma=\gamma^{\prime}
$$

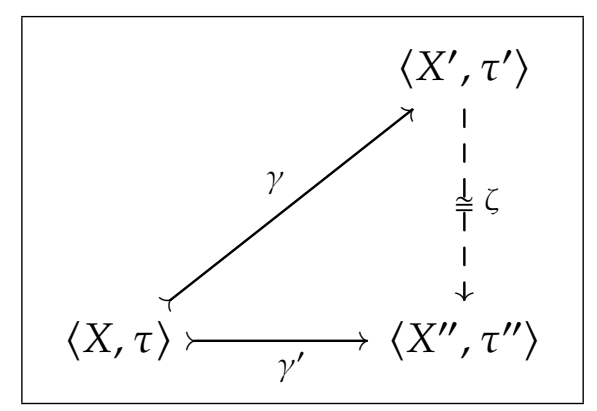

Diagrama iii.2

Com efeito: como $\gamma^{\prime}$ é contínua com respeito a $\tau$ e $\tau^{\prime \prime}$ e $\gamma$ é contínua com respeito a $\tau$ e $\tau^{\prime}$ (cf. Comentário 1.1.12 (viii) (2)), fixe então uma função contínua $\zeta: X^{\prime} \rightarrow X^{\prime \prime}$ com respeito a $\tau^{\prime}$ e $\tau^{\prime \prime}$ e uma função contínua $\zeta^{\prime}: X^{\prime \prime} \rightarrow X^{\prime}$ com respeito a $\tau^{\prime \prime}$ e $\tau^{\prime}$ tais que

$$
\zeta \circ \gamma=\gamma^{\prime} \text { e } \zeta^{\prime} \circ \gamma^{\prime}=\gamma
$$

Assim, tem-se que: 


$$
\begin{gathered}
\left(\zeta \circ \zeta^{\prime}\right) \circ \gamma^{\prime}=\zeta \circ\left(\zeta^{\prime} \circ \gamma^{\prime}\right)=\zeta \circ \gamma=\gamma^{\prime}=\operatorname{id}_{X^{\prime \prime}} \circ \gamma^{\prime} \\
\mathrm{e} \\
\left(\zeta^{\prime} \circ \zeta\right) \circ \gamma=\zeta^{\prime} \circ(\zeta \circ \gamma)=\zeta^{\prime} \circ \gamma^{\prime}=\gamma=\operatorname{id}_{X^{\prime}} \circ \gamma .
\end{gathered}
$$

Já que $\left\langle X^{\prime \prime}, \tau^{\prime \prime}\right\rangle$ e $\left\langle X^{\prime}, \tau^{\prime}\right\rangle$ são de Hausdorff (em virtude dos itens (v) (1) e (iv) (1) da Proposição 1.1.16), o conjunto $\operatorname{im}\left(\gamma^{\prime}\right)$ é um denso em $\left\langle X^{\prime \prime}, \tau^{\prime \prime}\right\rangle$ e o conjunto im $(\gamma)$ é um denso em $\left\langle X^{\prime}, \tau^{\prime}\right\rangle$, segue que:

$$
\zeta \circ \zeta^{\prime}=\mathrm{id}_{X^{\prime \prime}} \text { e } \zeta^{\prime} \circ \zeta=\mathrm{id}_{X^{\prime}}
$$

(cf. Proposição 1.1.21 (ii)), o que implica que $\zeta$ é um homeomorfismo com respeito a $\tau^{\prime}$ e $\tau^{\prime \prime}$. Além disso, note que, para toda função $\eta \in C\left(X^{\prime}, \tau^{\prime}, X^{\prime \prime}, \tau^{\prime \prime}\right)$, se $\eta \circ \gamma=\gamma^{\prime}$, então $\eta=\zeta$.

Teorema 3.1.3. Sejam $\langle X, \tau\rangle$ e $\left\langle X^{\prime}, \tau^{\prime}\right\rangle$ espaços topológicos de Tychonoff tais que $X$ e $X^{\prime}$ são não vazios e $\gamma: X \rightarrow X^{\prime}$ uma função tais que $\left\langle X^{\prime}, \tau^{\prime}, \gamma\right\rangle$ é uma compactificação maximal de $\langle X, \tau\rangle$. Então:

(i) Para toda função $\xi \in C_{b}(X, \tau)$, existe uma única função $\zeta \in C\left(X^{\prime}, \tau^{\prime}\right)$ tal que

$$
\zeta \circ \gamma=\xi
$$

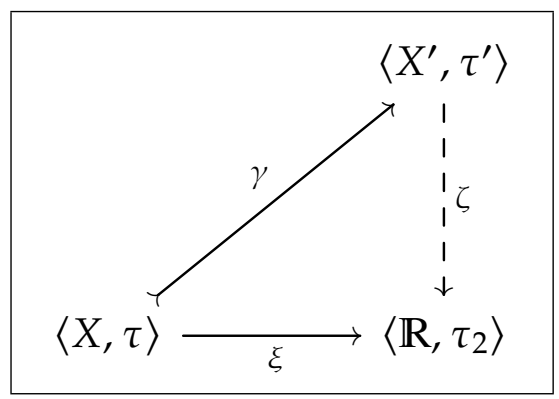

Diagrama iii.3

(ii) Existe um único isomorfismo isométrico $\Psi: C_{b}(X, \tau) \rightarrow C\left(X^{\prime}, \tau^{\prime}\right)$ segundo $|\cdot|_{\infty, X} e$ $|\cdot|_{\infty, X^{\prime}}$ tal que, para toda função $\xi \in C_{b}(X, \tau)$,

$$
\Psi(\xi) \circ \gamma=\xi
$$

Prova. (i) : Fixe arbitrariamente uma função contínua $\xi: X \rightarrow \mathbb{R}$ com respeito a $\tau$ e $\tau_{2}$ que é limitada segundo $d_{2}$. Como $X$ é não vazio, segue que o conjunto im( $\xi$ ) é não vazio. Fixe então um $\lambda \in \mathbb{R}$ e um $\delta \in \mathbb{R}^{+}$tais que $\operatorname{im}(\xi) \subseteq B_{d_{2}}[\lambda, \delta]=[\lambda-\delta, \lambda+\delta]$ (v. Proposição 1.2.15(ii)). Considere a função $\xi_{\lambda, \delta}:=\xi \downarrow[\lambda-\delta, \lambda+\delta]$. Sendo assim, $\xi_{\lambda, \delta}$ é contínua com respeito a $\tau$ e $\left(\tau_{2}\right)_{[\lambda-\delta, \lambda+\delta]}$. Sabe-se que $\left\langle[\lambda-\delta, \lambda+\delta],\left(\tau_{2}\right)_{[\lambda-\delta, \lambda+\delta]}\right\rangle$ é 


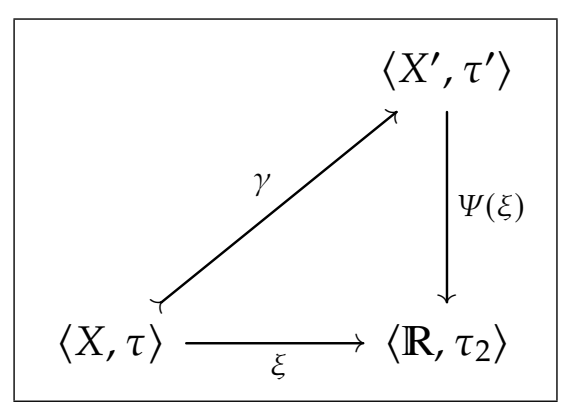

Diagrama iii.4

compacto (cf. Teorema 1.2.17 (i)). Além disso, tem-se que $\left\langle[\lambda-\delta, \lambda+\delta],\left(\tau_{2}\right)_{[\lambda-\delta, \lambda+\delta]}\right\rangle$ é de Tychonoff (em virtude do Comentário 1.2.5(ii) e das Proposições 1.2.10(iii) e 1.1.16(v)(2)). Fixe agora uma função contínua $\psi: X^{\prime} \rightarrow[\lambda-\delta, \lambda+\delta]$ com respeito a $\tau^{\prime}$ e $\left(\tau_{2}\right)_{[\lambda-\delta, \lambda+\delta]}$ tal que

$$
\psi \circ \gamma=\xi_{\lambda, \delta}
$$

Considere a função $\zeta_{\psi}: X^{\prime} \rightarrow \mathbb{R}$ que é definida pondo-se, para todo $x \in X^{\prime}$,

$$
\zeta_{\psi}(x):=\psi(x)
$$

É claro que $\operatorname{im}\left(\zeta_{\psi}\right)=\operatorname{im}(\psi)$ e que $\zeta_{\psi} \downarrow[\lambda-\delta, \lambda+\delta]=\psi$, o que implica que $\zeta_{\psi}$ é contínua com respeito a $\tau^{\prime}$ e $\tau_{2}$ (cf. Comentário 1.1.12(viii) (2) (1)). Note que:

$$
\zeta_{\psi} \circ \gamma=\xi
$$

Já que $\left\langle\mathbb{R}, \tau_{2}\right\rangle$ é de Hausdorff (cf. Comentário 1.1.15(iii)(2)) e o conjunto im( $\gamma$ ) é um denso em $\left\langle X^{\prime}, \tau^{\prime}\right\rangle$, segue que, para toda função $\eta \in C\left(X^{\prime}, \tau^{\prime}\right)$, se $\eta \circ \gamma=\xi$, então $\eta=\zeta_{\eta}$ (cf. Proposição 1.1.21 (ii)).

(ii) : Em virtude do item $(i)$, pode-se considerar a função $\Psi: C_{b}(X, \tau) \rightarrow C\left(X^{\prime}, \tau^{\prime}\right)$ que é definida da seguinte maneira: para toda função $\xi \in C_{b}(X, \tau), \Psi(\xi)$ é a única função contínua $\zeta: X^{\prime} \rightarrow \mathbb{R}$ com respeito a $\tau^{\prime}$ e $\tau_{2}$ tal que

$$
\zeta \circ \gamma=\xi
$$

Inicialmente, observe que: para toda função $H \in C\left(X^{\prime}, \tau^{\prime}\right)^{C_{b}(X, \tau)}$, se, para toda função $\xi \in C_{b}(X, \tau), H(\xi) \circ \gamma=\xi$, então $H=\Psi$. Tome funções $\xi, \xi^{\prime} \in C_{b}(X, \tau)$ e $\zeta \in C\left(X^{\prime}, \tau^{\prime}\right)$ quaisquer. Usando $(*)$, conclui-se que, se $\Psi(\xi)=\Psi\left(\xi^{\prime}\right)$, então

$$
\xi=\Psi(\xi) \circ \gamma=\Psi\left(\xi^{\prime}\right) \circ \gamma=\xi^{\prime} .
$$

Como $\gamma$ é contínua com respeito a $\tau$ e $\tau^{\prime}$ (cf. Comentário 1.1.12(x)(2)), tem-se que $\zeta \circ \gamma$ é contínua com respeito a $\tau$ e $\tau_{2}$. Já que $C\left(X^{\prime}, \tau^{\prime}\right)=C_{b}\left(X^{\prime}, \tau^{\prime}\right)$ (cf. Comentário 
1.2.36 (iv) (2)), tem-se que $\zeta \circ \gamma$ é limitada segundo $d_{2}$ (cf. Proposição 1.2.16(i)). Então, $\zeta \circ \gamma \in C_{b}(X, \tau) \cap \ell_{\infty}(X)=C_{b}(X, \tau)$. Usando $(*)$, conclui-se que:

$$
\Psi(\zeta \circ \gamma) \circ \gamma=\zeta \circ \gamma
$$

o que implica que $\Psi(\zeta \circ \gamma)=\zeta$. Como as funções $\xi, \xi^{\prime} \in C_{b}(X, \tau)$ e $\zeta \in C\left(X^{\prime}, \tau^{\prime}\right)$ foram tomadas quaisquer, segue que $\Psi$ é bijetora.

Agora, considere os conjuntos $C_{b}(X, \tau)$ e $C\left(X^{\prime}, \tau^{\prime}\right)$ munidos de suas estruturas canônicas de espaço vetorial sobre $\mathbb{R}$. Tome funções $\xi, \xi^{\prime} \in C_{b}(X, \tau)$ e um $\lambda \in \mathbb{R}$ quaisquer. Entáo, $\xi+\lambda \xi^{\prime} \in C_{b}(X, \tau)$. Usando $(*)$, conclui-se que:

$$
\begin{aligned}
\Psi\left(\xi+\lambda \xi^{\prime}\right) \circ \gamma=\xi+\lambda \xi^{\prime} & =\Psi(\xi) \circ \gamma+\lambda\left(\Psi\left(\xi^{\prime}\right) \circ \gamma\right)= \\
& =\Psi(\xi) \circ \gamma+\left(\lambda \Psi\left(\xi^{\prime}\right)\right) \circ \gamma= \\
& =\left(\Psi(\xi)+\lambda \Psi\left(\xi^{\prime}\right)\right) \circ \gamma .
\end{aligned}
$$

o que implica que $\Psi\left(\xi+\lambda \xi^{\prime}\right)=\Psi(\xi)+\lambda \Psi\left(\xi^{\prime}\right)$. Além disso, tem-se que:

$$
\{|\xi(x)|: x \in X\}=\{|(\Psi(\xi) \circ \gamma)(x)|: x \in X\}=\{|(\Psi(\xi))(\gamma(x))|: x \in X\},
$$

o que implica que

$$
\{|\xi(x)|: x \in X\}=\{|(\Psi(\xi))(z)|: z \in \operatorname{im}(\gamma)\}
$$

Então, tem-se que:

$$
|\xi|_{\infty, X}=\sup \{|\xi(x)|: x \in X\} \leqslant \sup \left\{|(\Psi(\xi))(z)|: z \in X^{\prime}\right\}=|\Psi(\xi)|_{\infty, X^{\prime}}
$$

Note que $|\Psi(\xi)|=|\cdot| \circ \Psi(\xi)$. Já que $|\cdot|$ é contínua com respeito a $\tau_{2}$ e $\tau_{2}$ (cf. Comentário $1.1 .15(i x))$, tem-se que $|\Psi(\xi)|$ é contínua com respeito a $\tau$ e $\tau_{2}$. Como $\overline{\operatorname{im}(\gamma)} \tau^{\prime}=X^{\prime}$, tem-se então que:

$$
|\Psi(\xi)|\left[X^{\prime}\right]=|\Psi(\xi)|\left[\overline{\operatorname{im}(\gamma)}^{\tau^{\prime}}\right] \subseteq \overline{|\Psi(\xi)|[\operatorname{im}(\gamma)}^{\tau_{2}}
$$

Usando (††) e (†), conclui-se que:

$$
\begin{aligned}
|\Psi(\xi)|_{\infty, X^{\prime}} & =\sup \left\{|(\Psi(\xi))(z)|: z \in X^{\prime}\right\}=\sup \left(|\Psi(\xi)|\left[X^{\prime}\right]\right) \leqslant \\
& \leqslant \sup \left(|\Psi(\xi)|[\operatorname{im}(\gamma)]^{\tau_{2}}\right)=\sup (|\Psi(\xi)|[\operatorname{im}(\gamma)])= \\
& =\sup \{|(\Psi(\xi))(z)|: z \in \operatorname{im}(\gamma)\}=\sup \{|\xi(x)|: x \in X\}=|\xi|_{\infty, X} .
\end{aligned}
$$


Logo, $|\Psi(\xi)|_{\infty, X^{\prime}}=|\xi|_{\infty, X}$. Já que as funções $\xi, \xi^{\prime} \in C_{b}(X, \tau)$ e $\lambda \in \mathbb{R}$ foram tomados quaisquer, então $\Psi$ é uma transformação linear que preserva comprimento segundo $|\cdot|_{\infty, X}$ e $|\cdot|_{\infty, X^{\prime}}$.

Portanto, $\Psi$ é um isomorfismo isométrico segundo $|\cdot|_{\infty, X}$ e $|\cdot|_{\infty, X^{\prime}}$

No que segue, são considerados os seguintes resultados: a Proposição 1.1.17 (i) (3) e o Teorema 1.1.24 (ii) (1).

Comentário 3.1.4. Dados um conjunto $I$, denotaremos por $D(I)$ o espaço topológico $\langle I, \mathcal{P}(I)\rangle$. Dados um espaço topológico $\left\langle X^{\prime}, \tau^{\prime}\right\rangle$ de Tychonoff e uma função $\gamma: I \rightarrow X^{\prime}$, suponha que $\left\langle X^{\prime}, \tau^{\prime}, \gamma\right\rangle$ seja uma quase compactificação de $D(I)$. Dada uma família $\left\{\left\langle X_{i}, \tau_{i}\right\rangle: i \in I\right\}$ de espaços topológicos de Tychonoff, um espaço topológico $\left\langle X^{\prime \prime}, \tau^{\prime \prime}\right\rangle$ de Tychonoff e uma função $\gamma^{\prime}: \sum_{i \in I} X_{i} \rightarrow X^{\prime \prime}$, suponha que $\left\langle X^{\prime \prime}, \tau^{\prime \prime}, \gamma^{\prime}\right\rangle$ seja uma compactificação maximal de $\sum_{i \in I}\left\langle X_{i}, \tau_{i}\right\rangle$. Lembre-se que $p_{I, X_{i}}$ denota a projeção de $\sum_{i \in I} X_{i}$ em I (cf. Comentário 1.1.22). Em virtude dos Comentários 1.1.12 (x) (2) e 1.1.22 (iii), tem-se que:

(i) A composição $\gamma \circ p_{I, X_{i}}$ é contínua com respeito a $\bigoplus_{i \in I} \tau_{i}$ e $\tau^{\prime}$.

Denote por $\mathcal{K}$ e por $\mathcal{K}^{\prime}$, respectivamente, os ternos $\left\langle X^{\prime}, \tau^{\prime}, \gamma\right\rangle$ e $\left\langle X^{\prime \prime}, \tau^{\prime \prime}, \gamma^{\prime}\right\rangle$. Em virtude

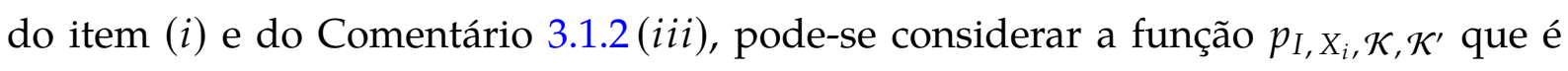
definida como a única função $\zeta \in C\left(X^{\prime \prime}, \tau^{\prime \prime}, X^{\prime}, \tau^{\prime}\right)$ tal que

$$
\zeta \circ \gamma^{\prime}=\gamma \circ p_{I, X_{i}}
$$

A função $p_{I, X_{i}}, \mathcal{K}, \mathcal{K}^{\prime}$ será chamada de o levantamento contínuo de $p_{I, X_{i}}$ com respeito a $\mathcal{K}$ e $\mathcal{K}^{\prime}$. Dada uma subfamília não vazia $\mathcal{F}$ de $\mathcal{P}(I)$, denote por $\sum_{I, \mathcal{F}, \mathcal{K}, \mathcal{K}^{\prime}} X_{i}$ o conjunto $\left(p_{I, X_{i}, \mathcal{K}, \mathcal{K}^{\prime}}\right)^{-1}\left[\bigcap_{J \in \mathcal{F}} \overline{\gamma[J]} \tau^{\tau^{\prime}}\right]$. Denote por $\bigoplus_{I, \mathcal{F}, \mathcal{K}, \mathcal{K}^{\prime}} \tau_{i}$ a topologia $\left(\tau^{\prime \prime}\right) \sum_{I, \mathcal{F}, \mathcal{K}, \mathcal{K}^{\prime}} X_{i} \cdot$ O espaço topológico $\left\langle\sum_{I, \mathcal{F}, \mathcal{K}, \mathcal{K}^{\prime}} X_{i}, \bigoplus_{I, \mathcal{F}, \mathcal{K}, \mathcal{K}^{\prime}} \tau_{i}\right\rangle$ será denotado por $\sum_{I, \mathcal{F}, \mathcal{K}, \mathcal{K}^{\prime}}\left\langle X_{i}, \tau_{i}\right\rangle$. Note que:

(ii) Se $\emptyset \in \mathcal{F}$, então $\sum_{I, \mathcal{F}, \mathcal{K}, \mathcal{K}^{\prime}} X_{i}=\emptyset$.

(iii) $\sum_{I, \mathcal{F}, \mathcal{K}, \mathcal{K}^{\prime}} X_{i}$ é um fechado em $\left\langle X^{\prime \prime}, \tau^{\prime \prime}\right\rangle$. 
Em virtude do item (iii) e da Proposição 1.1.13(i), tem-se que:

(iv) $\sum_{I, \mathcal{F}, \mathcal{K}, \mathcal{K}^{\prime}}\left\langle X_{i}, \tau_{i}\right\rangle$ é compacto.

Em virtude da Proposição 1.1.16 (v) (2), tem-se também que:

(v) $\sum_{I, \mathcal{F}, \mathcal{K}, \mathcal{K}^{\prime}}\left\langle X_{i}, \tau_{i}\right\rangle$ é de Tychonoff.

O teorema a seguir garante que a definição de ultrassoma topológica, que iremos apresentar mais à frente, não depende (a menos de homeomorfismos) da escolha que será feita para a compactificação maximal tanto da soma de uma família de espaços topológicos de Tychonoff quanto do espaço topológico discreto sobre o conjunto de índices dessa família.

Teorema 3.1.5. Sejam I um conjunto não vazio e $\mathcal{F}$ uma subfamília não vazia de $\mathcal{P}(I)$. Seja $\left\{\left\langle X_{i}, \tau_{i}\right\rangle: i \in I\right\}$ uma família de espaços topológicos de Tychonoff. Então, para quaisquer compactificações maximais $\mathcal{K}_{0}$ e $\mathcal{L}_{0}$ de $D(I)$ e quaisquer compactificações maximais $\mathcal{K}_{1}$ e $\mathcal{L}_{1}$ de $\sum_{i \in I}\left\langle X_{i}, \tau_{i}\right\rangle, \sum_{I, \mathcal{F}, \mathcal{K}_{0}, \mathcal{K}_{1}}\left\langle X_{i}, \tau_{i}\right\rangle$ é homeomorfo a $\sum_{I, \mathcal{F}, \mathcal{L}_{0}, \mathcal{L}_{1}}\left\langle X_{i}, \tau_{i}\right\rangle$.

Prova. Fixe arbitrariamente espaços topológicos $\left\langle X_{0}, \tau_{0}\right\rangle,\left\langle Z_{0}, \sigma_{0}\right\rangle,\left\langle X_{1}, \tau_{1}\right\rangle$ e $\left\langle Z_{1}, \sigma_{1}\right\rangle$ de Tychonoff e funções $\gamma_{0}: I \rightarrow X_{0}, \psi_{0}: I \rightarrow Z_{0}, \gamma_{1}: \sum_{i \in I} X_{i} \rightarrow X_{1}$ e $\psi_{1}: \sum_{i \in I} X_{i} \rightarrow Z_{1}$. Suponha que $\left\langle X_{0}, \tau_{0}, \gamma_{0}\right\rangle$ e $\left\langle Z_{0}, \sigma_{0}, \psi_{0}\right\rangle$ sejam compactificações maximais de $D(I)$ e que $\left\langle X_{1}, \tau_{1}, \gamma_{1}\right\rangle$ e $\left\langle Z_{1}, \sigma_{1}, \psi_{1}\right\rangle$ sejam compactificações maximais de $\sum_{i \in I}\left\langle X_{i}, \tau_{i}\right\rangle$. Denote por $\mathcal{K}_{0}$, por $\mathcal{L}_{0}$, por $\mathcal{K}_{1}$ e por $\mathcal{L}_{1}$, respectivamente, os ternos $\left\langle X_{0}, \tau_{0}, \gamma_{0}\right\rangle,\left\langle Z_{0}, \sigma_{0}, \psi_{0}\right\rangle$, $\left\langle X_{1}, \tau_{1}, \gamma_{1}\right\rangle$ e $\left\langle Z_{1}, \sigma_{1}, \psi_{1}\right\rangle$. Assim, tem-se que:

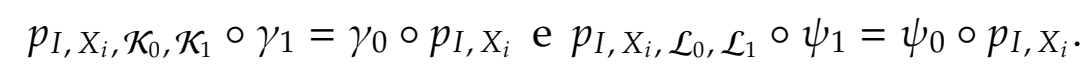

(v. Comentário 3.1.4). Fixe um homeomorfismo $\zeta_{0}: X_{0} \rightarrow Z_{0}$ com respeito a $\tau_{0}$ e $\sigma_{0}$ e um homeomorfismo $\zeta_{1}: X_{1} \rightarrow Z_{1}$ com respeito a $\tau_{1}$ e $\sigma_{1}$ tais que

$$
\zeta_{0} \circ \gamma_{0}=\psi_{0} \text { e } \zeta_{1} \circ \gamma_{1}=\psi_{1}
$$

(v. Comentário 3.1.2(iv)). Usando $(*)$ e $(* *)$, conclui-se que:

$$
\begin{aligned}
& \left(\zeta_{0} \circ p_{I, X_{i}}, \mathcal{K}_{0}, \mathcal{K}_{1}\right) \circ \gamma_{1}=\zeta_{0} \circ\left(p_{I, X_{i}}, \mathcal{K}_{0}, \mathcal{K}_{1} \circ \gamma_{1}\right)=\zeta_{0} \circ\left(\gamma_{0} \circ p_{I, X_{i}}\right)=\left(\zeta_{0} \circ \gamma_{0}\right) \circ p_{I, X_{i}}=
\end{aligned}
$$

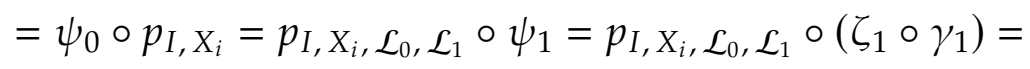

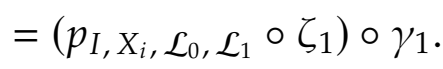


Já que $\left\langle Z_{0}, \sigma_{0}\right\rangle$ é de Hausdorff (em virtude dos itens (v)(1) e (iv) (1) da Proposição 1.1.16) e o conjunto $\operatorname{im}\left(\gamma_{1}\right)$ é um denso em $\left\langle X_{1}, \tau_{1}\right\rangle$, tem-se que:

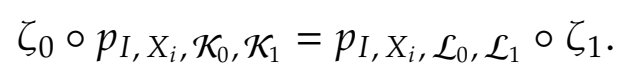

Como as funções $\zeta_{0}$ e $\zeta_{1}$ são bijetoras, tem-se então que:

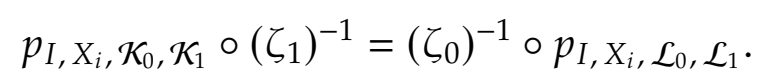

Tome um $J \in \mathcal{F}$ qualquer. Já que a função $\zeta_{0}$ é contínua e fechada com respeito a $\tau_{0}$ e $\sigma_{0}$, tem-se também que:

$$
\zeta_{0}\left[{\overline{\gamma_{0}[J]}}^{\tau_{0}}\right]={\overline{\zeta_{0}\left[\gamma_{0}[J]\right.}}^{\sigma_{0}}={\overline{\left(\zeta_{0} \circ \gamma_{0}\right)[J]}}^{\sigma_{0}}={\overline{\psi_{0}[J]}}^{\sigma_{0}}
$$

o que implica que

$$
\zeta_{0}\left[\bigcap_{J \in \mathcal{F}}{\overline{\gamma_{0}[J]}}^{\tau_{0}}\right]=\bigcap_{J \in \mathcal{F}}{\overline{\psi_{0}[J]}}^{\sigma_{0}}
$$

Usando (†) e (†t), conclui-se que:

$$
\begin{aligned}
& \zeta_{1}\left[\sum_{I, \mathcal{F}, \mathcal{K}_{0}, \mathcal{K}_{1}} X_{i}\right]=\left(\left(\zeta_{1}\right)^{-1}\right)^{-1}\left[\left(p_{I, X_{i}}, \mathcal{K}_{0}, \mathcal{K}_{1}\right)^{-1}\left[\bigcap_{J \in \mathcal{F}}{\overline{\gamma_{0}[J]}}^{\tau_{0}}\right]\right]= \\
& =\left(p_{I, X_{i}}, \mathcal{K}_{0}, \mathcal{K}_{1} \circ\left(\zeta_{1}\right)^{-1}\right)^{-1}\left[\bigcap_{J \in \mathcal{F}} \overline{\gamma_{0}[J]} \tau^{\tau_{0}}\right]=
\end{aligned}
$$

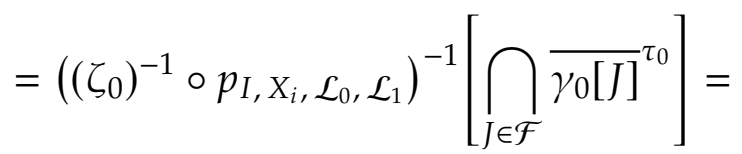

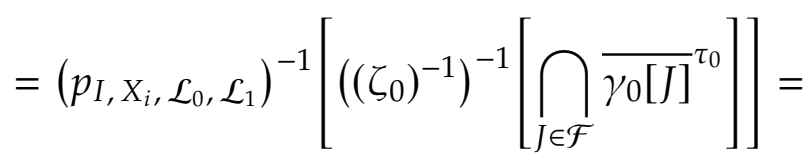

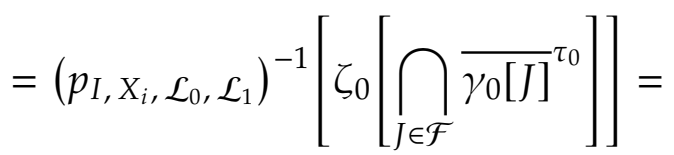

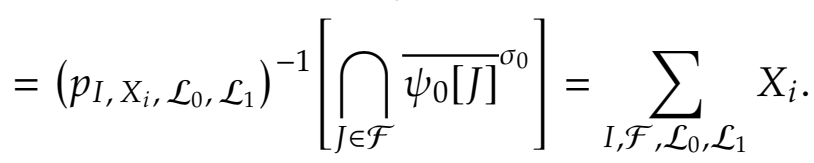

Então, tem-se que a função $\left(\zeta_{1} \uparrow \sum_{I, \mathcal{F}, \mathcal{K}_{0}, \mathcal{K}_{1}} X_{i}\right) \downarrow \sum_{I, \mathcal{F}, \mathcal{L}_{0}, \mathcal{L}_{1}} X_{i}$ é um homeomorfismo com respeito a $\bigoplus_{I, \mathcal{F}, \mathcal{K}_{0}, \mathcal{K}_{1}} \tau_{i}$ e $\bigoplus_{I, \mathcal{F}, \mathcal{L}_{0}, \mathcal{L}_{1}} \tau_{i}$ (cf. Comentário 1.1.12(ix)(2)). Portanto, conclui-se 
que $\sum_{I, \mathcal{F}, \mathcal{K}_{0}, \mathcal{K}_{1}}\left\langle X_{i}, \tau_{i}\right\rangle$ é homeomorfo a $\sum_{I, \mathcal{F}, \mathcal{L}_{0}, \mathcal{L}_{1}}\left\langle X_{i}, \tau_{i}\right\rangle$.

Vale salientar que a escrita da prova acima é de nossa autoria e que, na literatura a que tivemos acesso, não há menção alguma ao resultado no Teorema 3.1.5.

Comentário 3.1.6 ([BeS1969, p. 25-26], [CN1974, p. 24-25]). Dado um conjunto não vazio $I$, considere a seguinte família:

$$
\operatorname{Ult}(I):=\{\mathcal{U} \in \mathcal{P}(\mathcal{P}(I)): \mathcal{U} \text { é um ultrafiltro sobre } I\}
$$

Para cada $J \subseteq I$, considere a família $J^{\#}:=\{\mathcal{U} \in \operatorname{Ult}(I): J \in \mathcal{U}\}$. Para cada $\mathcal{A} \subseteq \mathcal{P}(I)$, considere a família $\mathcal{A}^{\#}:=\left\{J^{\#}: J \in \mathcal{A}\right\}$. Lembre-se que, para cada $i \in I, \mathcal{U}(i)$ denota o ultrafiltro principal sobre $I$ em $i$ (cf. Comentário 1.1.2). Note que:

(i) (1) Para todo $J \subseteq I$,

(1) $\{\mathcal{U}(i): i \in J\} \subseteq J^{\#}$.

(2) $J^{\#}=\emptyset$ se, e somente se, $J=\emptyset$.

(3) $J^{\#}=\operatorname{Ult}(I)$ se, e somente se, $J=I$.

(4) $(I \backslash J)^{\#}=\operatorname{Ult}(I) \backslash J^{\#}$.

(2) Para quaisquer $J, J^{\prime} \subseteq I$,

(1) $\left(J \cap J^{\prime}\right)^{\#}=J^{\#} \cap\left(J^{\prime}\right)^{\#}$.

(2) $\left(J \cup J^{\prime}\right)^{\#}=J^{\#} \cup\left(J^{\prime}\right)^{\#}$.

(3) $\left(J \backslash J^{\prime}\right)^{\#}=J^{\#} \backslash\left(J^{\prime}\right)^{\#}$.

(3) Para todo $\mathcal{A} \subseteq \mathcal{P}(I)$,

(1) $\cup \mathcal{A}^{\#}=\{\mathcal{U} \in \operatorname{Ult}(I): \mathcal{U} \cap \mathcal{A} \neq \emptyset\}$.

(2) se $\mathcal{A}$ for não vazio, então $\mathcal{A}^{\#}$ é não vazio e

$$
\cap \mathcal{A}^{\#}=\{\mathcal{U} \in \operatorname{Ult}(I): \mathcal{A} \subseteq \mathcal{U}\} .
$$

Para cada $\mathscr{A} \subseteq \mathcal{P}(\mathcal{P}(\mathcal{P}(I)))$, considere a família ${ }^{\#} \mathscr{A}:=\left\{J \in \mathcal{P}(I): J^{\#} \in \mathscr{A}\right\}$. Usando os itens $(i)$ (1) (2) e (i) (2) (1), é corriqueiro mostrar que:

(ii) (1) Para todo $\mathcal{A} \subseteq \mathcal{P}(I)$,

(1) $\mathcal{A}^{\#} \subseteq \mathcal{P}(\mathcal{P}(\mathcal{P}(I))) \mathrm{e}^{\#}\left(\mathcal{A}^{\#}\right)=\mathcal{A}$.

(2) se $\mathcal{A}$ tiver a PIF, então $\mathcal{A}^{\#}$ tem a PIF.

(2) Para todo $\mathscr{A} \subseteq \mathcal{P}(\mathcal{P}(\mathcal{P}(I)))$, se $\mathscr{A}$ tiver a PIF, então ${ }^{\#} \mathscr{A}$ tem a PIF. 
Com o uso dos itens (i) (1) (3) e (i) (2) (1) e do Comentário 1.1.10 (ii), conclui-se que:

(iii) $\mathcal{P}(I)^{\#}$ gera uma topologia sobre $\operatorname{Ult}(I)$.

A topologia gerada $\left(\mathcal{P}(I)^{\#}\right) \cup$ será chamada de a topologia de Stone sobre Ult $(I)$ e denotada por $\sigma(I)$. O espaço topológico $\langle\operatorname{Ult}(I), \sigma(I)\rangle$ será chamado de o espaço de Stone sobre $I$ e denotado por $S(I)$. Usando o Comentário 1.1.2(v), é fácil verificar que:

(iv) (1) Para todo $i \in I,\{i\}^{\#}=\{\mathcal{U}(i)\}$.

(2) Para todo $J \subseteq I,\{\mathcal{U}(i): i \in J\} \in \sigma(I)$.

(3) Para todo $\mathcal{U} \in \operatorname{Ult}(I), \mathcal{U}$ é um ponto isolado de $S(I)$ se, e somente se, $\mathcal{U}$ for um ultrafiltro principal.

Com o uso do item (i)(1) (4), do Comentário 1.1.3 (vii) e da Proposição 1.1.17(ii)(2), conclui-se que:

(v) (1) (1) Para todo $J \subseteq I, J^{\#}$ é um fechado em $S(I)$.

(2) $S(I)$ é zero-dimensional.

(2) (1) Para quaisquer $\mathcal{U}, \mathcal{U}^{\prime} \in \operatorname{Ult}(I)$, se $\mathcal{U} \neq \mathcal{U}^{\prime}$, então existe um $J \subseteq I$ tal que $\mathcal{U} \in J^{\#}$ e $\mathcal{U}^{\prime} \notin J^{\#}$.

(2) $S(I)$ é $T_{1}$.

(3) $S(I)$ é de Tychonoff.

Considere a função $\operatorname{prin}_{I}: I \rightarrow \operatorname{Ult}(I)$ que é definida pondo-se, para todo $i \in I$,

$$
\operatorname{prin}_{I}(i):=\mathcal{U}(i)
$$

Note que:

(vi) (1) $\operatorname{prin}_{I}$ é injetora.

(2) prin $_{I}$ é contínua com respeito a $\mathcal{P}(I)$ e $\sigma(I)$.

Em virtude dos itens (iv) (2) e (vi) e do Comentário 1.1.12 (x) (2), tem-se que:

(vii) (1) $\operatorname{prin}_{I}$ é aberta com respeito a $\mathcal{P}(I)$ e $\sigma(I)$.

(2) prin $_{I}$ é uma imersão topológica com respeito a $\mathcal{P}(I)$ e $\sigma(I)$.

Afirmamos que:

(viii) (1) Para todo $J \subseteq I, \overline{\operatorname{prin}}[I J]^{\sigma(I)}=J^{\#}$. 
(2) O conjunto im $\left(\operatorname{prin}_{I}\right)$ é um denso em $S(I)$.

De fato:

(1) : Fixe arbitrariamente um $J \subseteq I$. Usando os itens (i) (1) (1) e (v) (1) (1), conclui-se que ${\overline{\operatorname{prin}_{I}[J]}}^{\sigma(I)} \subseteq J^{\#}$. Tome um $\mathcal{U} \in J^{\#}$ qualquer e um $J^{\prime} \subseteq I$ tal que $\mathcal{U} \in\left(J^{\prime}\right)^{\#}$. Assim, tem-se que o conjunto $J^{\#} \cap\left(J^{\prime}\right)^{\#}$ é não vazio. Usando os itens (i) (2) (1) e (i) (1) (2), conclui-se que o conjunto $J \cap J^{\prime}$ é não vazio. Fixe um $j \in J \cap J^{\prime}$. Em virtude do item (i)(1)(1), tem-se que $\operatorname{prin}_{I}(j) \in\left(J^{\prime}\right)^{\#}$, o que implica que o conjunto $\left(J^{\prime}\right)^{\#} \cap \operatorname{prin}_{I}[J]$ é não vazio. Já que $\mathcal{P}(I)^{\#}$ é uma base para $\sigma(I)$, conclui-se usando o Comentário 1.1.10 (iii) (1) que $\mathcal{U} \in \overline{\operatorname{prin}}[I J]^{\sigma(I)}$. Como $\mathcal{U} \in J^{\#}$ foi tomado qualquer, então $J^{\#} \subseteq{\overline{\operatorname{prin}_{I}[J]}}^{\sigma(I)}$.

(2) : Usando (1) e o item (i) (1) (3), tem-se que:

$$
{\overline{\operatorname{im}\left(\operatorname{prin}_{I}\right)}}^{\sigma(I)}=\overline{\operatorname{prin}}[I]^{\sigma(I)}=I^{\#}=\operatorname{Ult}(I) .
$$

Afirmamos que:

(ix) Para todo espaço topológico $\langle Z, \sigma\rangle$ compacto e de Tychonoff e toda função $\xi \in Z^{I}$, existe uma função $\zeta \in C(\operatorname{Ult}(I), \sigma(I), Z, \sigma)$ tal que

$$
\zeta \circ \operatorname{prin}_{I}=\xi \text {. }
$$

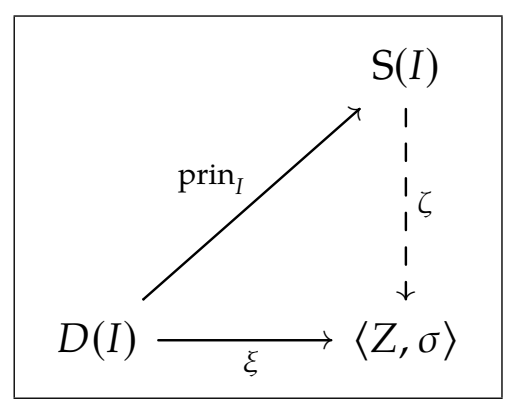

Diagrama iii.5

Com efeito: fixe arbitrariamente um espaço topológico $\langle Z, \sigma\rangle$ compacto e de Tychonoffe uma função $\xi: I \rightarrow Z$. Já que $\{\xi(i): i \in I\} \subseteq Z$ e $\langle Z, \sigma\rangle$ é de Hausdorff (em virtude dos itens $(v)(1)$ e (iv) (1) da Proposição 1.1.16), tem-se que existe um único $\mathcal{U}$-limite de $\xi$ com respeito a $\sigma$ (cf. Corolário 2.1.14). Logo, pode-se considerar a função $\zeta: \operatorname{Ult}(I) \rightarrow Z$ que é definida pondo-se, para todo $\mathcal{U} \in \operatorname{Ult}(I)$,

$$
\zeta(\mathcal{U}):=\mathcal{U}-\lim _{i \in I}^{\sigma} \xi(i)
$$

Como, para todo $j \in I, \mathcal{U}(j)-\lim _{i \in I}^{\sigma} \xi(i)=\xi(j)$ (cf. Comentário 2.1.5 (v)), segue que:

$$
\zeta \circ \operatorname{prin}_{I}=\xi
$$


Fixe arbitrariamente um $\mathcal{U} \in \operatorname{Ult}(I)$ e uma vizinhança aberta $V$ de $\zeta(\mathcal{U})$ em $\langle Z, \sigma\rangle$. Já que $\langle Z, \sigma\rangle$ é regular, $Z \backslash V$ é um fechado em $\langle Z, \sigma\rangle$ e $\zeta(\mathcal{U}) \notin Z \backslash V$, fixe $W, W^{\prime} \in \sigma$ tais que $\zeta(\mathcal{U}) \in W$,

$$
Z \backslash V \subseteq W^{\prime} \text { e } W \cap W^{\prime}=\emptyset
$$

Para cada $A \subseteq Z$, considere o conjunto $J_{A}:=\{i \in I: \xi(i) \in A\}$. Note que $J_{W} \in \mathcal{U}$, o que equivale a $\mathcal{U} \in\left(J_{W}\right)^{\#}$. Note também que, para quaisquer $A, A^{\prime} \subseteq Z, J_{Z \backslash A}=I \backslash J_{A}$ e $J_{A} \subseteq J_{A^{\prime}}$. Suponha que $\zeta\left[\left(J_{W}\right)^{\#}\right] \nsubseteq V$. Fixe então um $z \in \zeta\left[\left(J_{W}\right)^{\#}\right] \backslash Z$. Fixe agora um $\mathcal{U}^{\prime} \in\left(J_{W}\right)^{\#}$ tal que $z=\zeta\left(\mathcal{U}^{\prime}\right)$. Logo, $J_{W} \in \mathcal{U}^{\prime}$ e $\zeta\left(\mathcal{U}^{\prime}\right) \in Z \backslash V$. Usando $(*)$, conclui-se que:

$$
\zeta\left(\mathcal{U}^{\prime}\right) \in W^{\prime} \subseteq Z \backslash W
$$

o que implica que $J_{W^{\prime}} \in \mathcal{U}^{\prime}$ e que $J_{W^{\prime}} \subseteq J_{Z \backslash W}=I \backslash J_{W}$. Como $\mathcal{U}^{\prime}$ é um filtro sobre $I$, segue que $I \backslash J_{W} \in \mathcal{U}^{\prime}$. Então,

$$
\emptyset=J_{W} \cap\left(I \backslash J_{W}\right) \in \mathcal{U}^{\prime}
$$

uma contradição. Logo, $\zeta\left[\left(J_{W}\right)^{\#}\right] \subseteq V$. Já que foram fixados arbitrariamente $\mathcal{U} \in \operatorname{Ult}(I)$ e a vizinhança aberta $V$ de $\zeta(\mathcal{U})$ em $\langle Z, \sigma\rangle$, conclui-se que $\zeta$ é contínua com respeito a $\sigma(I)$ e $\sigma$.

Em virtude dos itens (v) (3), (vii) (2), (viii) (2) e (ix), tem-se que:

(x) Se S(I) for compacto, então $S(I)$ é um compactificado maximal de $D(I)$ segundo $\operatorname{prin}_{I}$.

Caso $S(I)$ seja compacto, o terno $\left\langle\operatorname{Ult}(I), \sigma(I), \operatorname{prin}_{I}\right\rangle$ será chamado de a compactificação usual de $D(I)$.

Teorema 3.1.7. Em ZF, são equivalentes:

(i) UT.

(ii) Para todo conjunto não vazio I, S(I) é compacto.

(iii) Para todo conjunto não vazio $I,\left\langle\mathrm{Ult}(I), \sigma(I)\right.$, prin $\left._{I}\right\rangle$ é uma compactificação maximal de $D(I)$.

Prova. $(i) \Longrightarrow($ ii $)$ : Provaremos por redução ao absurdo: suponha que $(i)$ valha e que (ii) não valha. Fixe um conjunto não vazio $I$ tal que $S(I)$ não é compacto. Assim, tem-se que, para toda base $\mathcal{B}$ para $\sigma(I)$, existe um $C \subseteq \mathcal{B}$ tal que $C$ é uma cobertura de Ult $(I)$ e 
toda subfamília finita de $C$ não é uma cobertura de Ult $(I)$ (v. Proposição 1.1.11). Já que $\mathcal{P}(I)^{\#}$ é uma base para $\sigma(I)$, fixe agora um $\mathscr{C} \subseteq \mathcal{P}(\mathrm{Ult}(I))$ tal que

$$
\mathscr{C} \subseteq \mathcal{P}(I)^{\#} \text { e } \mathscr{C} \text { é uma cobertura de } \operatorname{Ult}(I)
$$

Toda subfamília finita de $\mathscr{C}$ não é uma cobertura de Ult $(I)$.

Para cada $\mathscr{A} \subseteq \mathcal{P}(\mathrm{Ult}(I))$, considere a seguinte família:

$$
\mathscr{A}^{*}:=\{\operatorname{Ult}(I) \backslash \mathcal{A}: \mathcal{A} \in \mathscr{A}\} .
$$

Mostraremos que $\mathscr{C}^{*}$ tem a PIF: tome um $\mathscr{A} \subseteq \mathscr{C}^{*}$ qualquer. Suponha que $\mathscr{A}$ seja não vazio e finito. É claro que $\mathscr{A}^{*} \subseteq \mathscr{C}$ e que $\mathscr{A}^{*}$ é não vazio e finito. Usando (**), conclui-se que $\mathscr{A}^{*}$ não é uma cobertura de Ult(I). Assim, tem-se que:

$$
\cap \mathscr{A}=\bigcap_{\mathcal{A} \in \mathscr{A}} \mathcal{A}=\operatorname{Ult}(I) \backslash \bigcup_{\mathcal{A} \in \mathscr{A}}(\operatorname{Ult}(I) \backslash \mathcal{A})=\operatorname{Ult}(I) \backslash \bigcup \mathscr{A}^{*} \neq \emptyset .
$$

Logo, ${ }^{*}\left(\mathscr{C}^{*}\right)$ tem a PIF (cf. Comentário 3.1.6(ii)(2)). Fixe então um $\mathcal{U} \in \operatorname{Ult}(I)$ tal que ${ }^{\#}\left(\mathscr{C}^{*}\right) \subseteq \mathcal{U}$ (v. Teorema 1.1.6). Usando (*), fixe agora um $J \subseteq I$ tal que $\mathcal{U} \in J^{\#} \in \mathscr{C}$. Assim, tem-se que $J \in \mathcal{U}$. Como $(I \backslash J)^{\#}=\operatorname{Ult}(I) \backslash J^{\#}$ (cf. Comentário 3.1.6(i)(1) (4)), tem-se também que $(I \backslash J)^{\#} \in \mathscr{C}^{*}$, o que implica que $I \backslash J \in{ }^{\#}\left(\mathscr{C}^{*}\right)$. Então,

$$
\emptyset=J \cap(I \backslash J) \in \mathcal{U}
$$

uma contradição.

(ii) $\Longrightarrow($ i $)$ : Fixe arbitrariamente um conjunto não vazio $I$ e um $\mathcal{A} \subseteq \mathcal{P}(I)$. Suponha que $\mathcal{A}$ seja não vazio e tenha a PIF. Então, a família $\mathcal{A}^{\#}$ é não vazia, tem a PIF e

$$
\cap \mathcal{A}^{\#}=\{\mathcal{U} \in \operatorname{Ult}(I): \mathcal{A} \subseteq \mathcal{U}\}
$$

(em virtude dos itens (i) (3) (2) e (ii) (1) (2) de Comentário 3.1.6). Além disso, tem-se que todo membro de $\mathcal{A}^{\#}$ é um fechado em S(I) (cf. Comentário 3.1.6(v) (1) (1)). Suponha que (ii) valha. Então, o conjunto $\cap \mathcal{A}^{\#}$ é não vazio (v. Proposição 1.1.11). Logo, existe um ultrafiltro $\mathcal{U}$ sobre $I$ tal que $\mathcal{A} \subseteq \mathcal{U}$. Portanto, conclui-se usando o Teorema 1.1.6 que (i) vale.

(ii) $\Longrightarrow($ iii $)$ : É imediato do Comentário 3.1.6(x).

(iii) $\Longrightarrow($ ii) : É óbvio.

Uma consequência imediata do Comentário 1.2.36 (iii) e dos Teoremas 3.1.3 e 3.1.7 é o seguinte 
Corolário 3.1.8. Seja I um conjunto não vazio. Assumindo-se UT, tem-se que:

(i) Para toda função $\xi \in \ell_{\infty}(I)$, existe uma única função $\zeta \in C(\operatorname{Ult}(I), \sigma(I))$ tal que

$$
\zeta \circ \operatorname{prin}_{I}=\xi \text {. }
$$

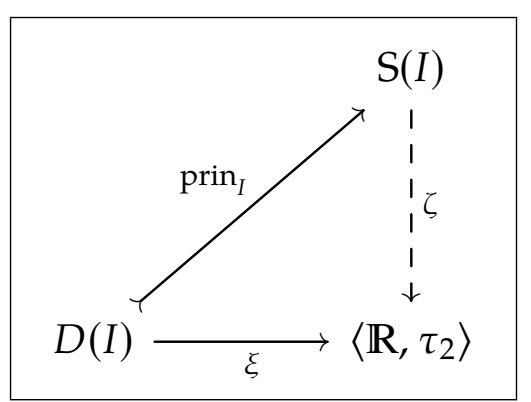

Diagrama iii.6

(ii) Existe um único isomorfismo isométrico $\Psi: \ell_{\infty}(I) \rightarrow C(\operatorname{Ult}(I), \sigma(I))$ segundo $|\cdot|_{\infty, X} e$ $|\cdot|_{\infty, \mathrm{Ult}(I)}$ tal que, para toda função $\xi \in \ell_{\infty}(I)$,

$$
\Psi(\xi) \circ \operatorname{prin}_{I}=\xi .
$$

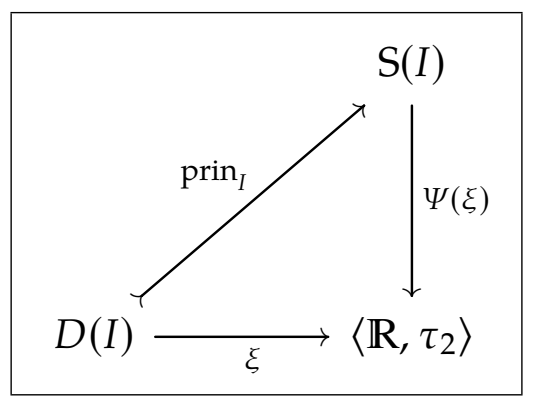

Diagrama iii.7

Comentário 3.1.9 ([Eng1989, cap. 3, seç. 5], [Sch1997, cap. 17, seç. 22]). Dado um espaço topológico $\langle X, \tau\rangle$, com o uso do Comentário 1.2.5(ii), das Proposições 1.2.10(iii) e 1.1.16(v) (2) e do Teorema 1.1.24 (ii) (2), conclui-se que:

(i) A potência topológica $\left\langle[0,1],\left(\tau_{2}\right)_{[0,1]}\right\rangle^{C\left(X, \tau,[0,1],\left(\tau_{2}\right)_{[0,1]}\right)}$ é de Tychonoff.

Considere a função $\Delta_{X}: X \rightarrow[0,1]^{C\left(X, \tau,[0,1],\left(\tau_{2}\right)_{[0,1]}\right)}$ tal que, para todo $x \in X, \Delta_{X}(x)$ é a função ev $v_{x}: C\left(X, \tau,[0,1],\left(\tau_{2}\right)_{[0,1]}\right) \rightarrow[0,1]$ que é definida pondo-se, para toda função $\xi \in C\left(X, \tau,[0,1],\left(\tau_{2}\right)_{[0,1]}\right)$,

$$
\mathrm{ev}_{x}(\xi):=\xi(x)
$$

A função $\Delta_{X}$ é chamada de a função diagonal de $C\left(X, \tau,[0,1],\left(\tau_{2}\right)_{[0,1]}\right)$. Para cada $x \in X$, a função ev $x$ é chamada de a função valoração de $C\left(X, \tau,[0,1],\left(\tau_{2}\right)_{[0,1]}\right)$ em $x$. Suponha que $\langle X, \tau\rangle$ seja de Tychonoff. Prova-se que: 
(ii) $\Delta_{X}$ é uma imersão topológica com respeito a $\tau$ e $\left(\left(\tau_{2}\right)_{[0,1]}\right)\left(X, \tau,[0,1],\left(\tau_{2}\right)_{[0,1]}\right)$.

Assuma UT. Prova-se que:

(iii) A potência topológica $\left\langle[0,1],\left(\tau_{2}\right)_{[0,1]}\right\rangle^{C\left(X, \tau,[0,1],\left(\tau_{2}\right)_{[0,1]}\right)}$ é compacta.

Em virtude dos itens (i), (ii) e (iii), tem-se que:

(iv) A potência topológica $\left\langle[0,1],\left(\tau_{2}\right)_{[0,1]}\right\rangle^{C\left(X, \tau,[0,1],\left(\tau_{2}\right)_{[0,1]}\right)}$ é um quase compactificado de $\langle X, \tau\rangle$ segundo $\Delta_{X}$.

Denote por $\beta X$, por $\beta \tau$ e por $\beta_{X}$, respectivamente, o conjunto $\overline{\Delta_{X}} X$, a topologia $\overline{\Delta_{X}} \tau$ e a função $\overline{\Delta_{X}}$ (v. Comentário 3.1.2). O espaço topológico $\langle\beta X, \beta \tau\rangle$ será denotado por $\beta\langle X, \tau\rangle$. Em virtude do Comentário 3.1.2 (iii), tem-se que:

(v) $\beta\langle X, \tau\rangle$ é um compactificado de $\langle X, \tau\rangle$ segundo $\beta_{X}$.

O espaço topológico $\beta\langle X, \tau\rangle$ será chamado de o compactificado de Stone-Čech de $\langle X, \tau\rangle$ segundo $\beta_{X}$. O terno $\left\langle\beta X, \beta \tau, \beta_{X}\right\rangle$ será chamado de a compactificação de Stone-Čech de $\langle X, \tau\rangle$. Prova-se que:

(vi) $\beta\langle X, \tau\rangle$ é um compactificado maximal de $\langle X, \tau\rangle$ segundo $\beta_{X}$.

Em virtude do item (vi), do Teorema 3.1.7 e do Comentário 3.1.2(iv), tem-se que:

(vii) Para todo conjunto não vazio $I$, existe um único homeomorfismo $\zeta: \operatorname{Ult}(I) \rightarrow \beta I$ com respeito a $\sigma(I)$ e $\beta \mathcal{P}(I)$ tal que

$$
\zeta \circ \operatorname{prin}_{I}=\beta_{I}
$$

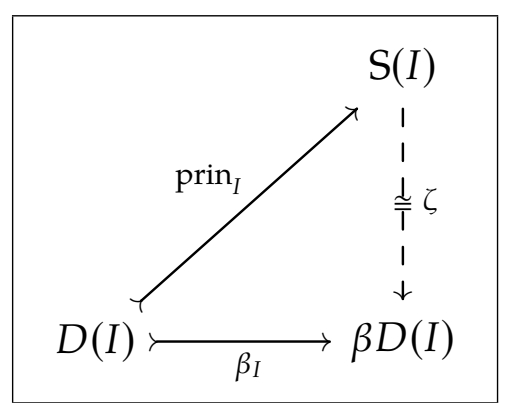

Diagrama iii.8

Definição 3.1.10. Sejam $I$ um conjunto não vazio e $\mathcal{F}$ um filtro sobre $I$. Seja $\left\{\left\langle X_{i}, \tau_{i}\right\rangle: i \in I\right\}$ uma família de espaços topológicos de Tychonoff. Seja $\langle X, \tau\rangle$ um espaço topológico de Tychonoff. Assuma UT. Denote por $\mathcal{K}_{\mathrm{S}}(I)$ a compactificação 
usual de $D(I)$ e por $\mathcal{K}_{\beta}\left(I, \tau_{i}\right)$ a compactificação de Stone-Čech de $\sum_{i \in I}\left\langle X_{i}, \tau_{i}\right\rangle$.

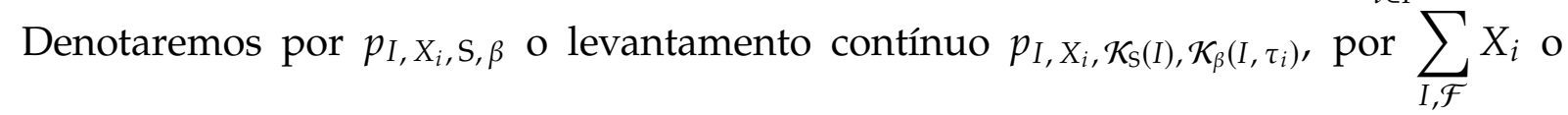
conjunto $\sum_{I, \mathcal{F}, \mathcal{K}_{\mathrm{S}}(I), \mathcal{K}_{\beta}\left(I, \tau_{i}\right)} X_{i}$ e por $\bigoplus_{I, \mathcal{F}} \tau_{i}$ a topologia $\bigoplus_{I, \mathcal{F}, \mathcal{K}_{\mathrm{S}}(I), \mathcal{K}_{\beta}\left(I, \tau_{i}\right)} \tau_{i}$. O espaço topológico $\sum_{I, \mathcal{F}, \mathcal{K}_{S}(I), \mathcal{K}_{\beta}\left(I, \tau_{i}\right)}\left\langle X_{i}, \tau_{i}\right\rangle$ será denotado por $\sum_{I, \mathcal{F}}\left\langle X_{i}, \tau_{i}\right\rangle$. Diremos que:

(i) $p_{I, X_{i}, \mathrm{~S}, \beta}$ é o levantamento de Stone-Čech de $p_{I, X_{i}}$.

(ii) $\sum_{I, \mathcal{F}}\left\langle X_{i}, \tau_{i}\right\rangle$ é a soma reduzida (topológica) de $\left\{\left\langle X_{i}, \tau_{i}\right\rangle: i \in I\right\}$ com respeito a $\mathcal{F}$. Se $\mathcal{F}$ for um ultrafiltro sobre $I$, diremos que $\sum_{I, \mathscr{F}}\left\langle X_{i}, \tau_{i}\right\rangle$ é a ultrassoma (topológica) de $\left\{\left\langle X_{i}, \tau_{i}\right\rangle: i \in I\right\}$ com respeito a $\mathcal{F} .^{1}$

Suponhamos que, para cada $i \in I,\left\langle X_{i}, \tau_{i}\right\rangle=\langle X, \tau\rangle$. Denotaremos por $(I, \mathcal{F}) X$ (ou, simplesmente, por $\mathcal{F} X)$ o conjunto $\sum_{I, \mathcal{F}} X_{i}$ e por $(I, \mathcal{F}) \tau$ (ou, simplesmente, por $\mathcal{F} \tau$ ) a topologia $\bigoplus_{I, \mathcal{F}} \tau_{i}$. O espaço topológico $\sum_{I, \mathcal{F}}\left\langle X_{i}, \tau_{i}\right\rangle$ será denotado por $(I, \mathcal{F})\langle X, \tau\rangle$ (ou, simplesmente, por $\mathcal{F}\langle X, \tau\rangle)$. Diremos que:

(iii) $(I, \mathcal{F})\langle X, \tau\rangle$ é o múltiplo reduzido (topológico) de $\langle X, \tau\rangle$ com respeito a $\mathcal{F}$. Se $\mathcal{F}$ for um ultrafiltro sobre $I$, diremos que $(I, \mathcal{F})\langle X, \tau\rangle$ é o ultramúltiplo (topológico) de $\langle X, \tau\rangle$ com respeito a $\mathcal{F}$.

Encerraremos esta seção com o corolário a seguir, que é imediato do Comentário 1.1.3 (vii), dos itens (viii) (1) e (i) (3) (2) do Comentário 3.1.6 e dos itens (iii), (iv) e (v) do Comentário 3.1.4.

Corolário 3.1.11. Sejam I um conjunto não vazio, $\mathcal{F}$ um filtro sobre I e $\left\{\left\langle X_{i}, \tau_{i}\right\rangle: i \in I\right\}$ uma família de espaços topológicos de Tychonoff. Assumindo-se UT, tem-se que:

(i) (1) $\sum_{I, \mathcal{F}} X_{i}=\left(p_{I, X_{i}, \mathrm{~S}, \beta}\right)^{-1}[\{\mathcal{U} \in \operatorname{Ult}(I): \mathcal{F} \subseteq \mathcal{U}\}]$.

(2) Se $\mathcal{F}$ for um ultrafiltro sobre I, então $\sum_{I, \mathcal{F}} X_{i}=\left(p_{I, X_{i}, \mathrm{~S}, \beta}\right)^{-1}[\{\mathcal{F}\}]$.

${ }^{1}$ Em [Ban1987, Gur1988], são utilizadas originalmente as expressões coproduto reduzido topológico e ultracoproduto topológico em lugar das nossas expressões soma reduzida topológica e ultrassoma topológica, respectivamente. As expressões originais são justificadas pelo contexto categorial no qual se introduziu as noções que cada uma nomeia. 
(ii) (1) $\sum_{I, \mathcal{F}} X_{i}$ é um fechado em $\beta \sum_{i \in I}\left\langle X_{i}, \tau_{i}\right\rangle$.

(2) $\sum_{I, \mathcal{F}}\left\langle X_{i}, \tau_{i}\right\rangle$ é compacto.

(iii) $\sum_{I, \mathcal{F}}\left\langle X_{i}, \tau_{i}\right\rangle$ é de Tychonoff.

\subsection{Ultraprodutos normados de espaços de funções reais contínuas e limitadas que estão definidas em espaços topológicos de Tychonoff}

Nesta seção, prova-se, em ZFC, uma generalização da Proposição 2 de [Gur1988] no contexto dos espaços topológicos de Tychonoff. No que segue, são apresentados enunciados precisos - sem demonstração - de tal proposição e de um resultado que será utilizado para provar essa generalização.

Teorema 3.2.1 (Lema 3.1 de [Ban1984]). Sejam I um conjunto não vazio, $\mathcal{U}$ um ultrafiltro sobre I e $\left\{\left\langle X_{i}, \tau_{i}\right\rangle: i \in I\right\}$ uma família de espaços topológicos de Tychonoff. Assumindo-se AC, tem-se que $\sum_{I, \mathcal{U}}\left\langle X_{i}, \tau_{i}\right\rangle$ é homeomorfa a $\sum_{I, \mathcal{U}} \beta\left\langle X_{i}, \tau_{i}\right\rangle$.

Teorema 3.2.2 (Proposição 2 de [Gur1988]). Sejam I um conjunto não vazio, $\mathcal{U}$ um ultrafiltro sobre I e $\left\{\left\langle K_{i}, \tau_{i}\right\rangle: i \in I\right\}$ uma família de espaços topológicos não vazios, compactos e de Hausdorff. Assumindo-se AC, tem-se que $\prod_{I, \mathcal{U}}\left\langle C\left(K_{i}, \tau_{i}\right),|\cdot|_{\infty}, K_{i}\right\rangle$ é isometricamente isomorfo a $\left\langle C\left(\sum_{I, \mathcal{U}} K_{i}, \bigoplus_{I, \mathcal{U}} \tau_{i}\right),|\cdot|_{\infty, \sum_{I, \mathcal{U}} K_{i}}\right\rangle$.

Vale salientar que os resultados nos Teoremas 3.2.1 e 3.2.2 foram demonstrados no contexto ingênuo ${ }^{2}$ dos espaços topológicos e que estamos assumindo AC no enunciado de ambos por não sabermos ainda se é suficiente assumir UT para prová-los. Por isto, é natural que façamos a seguinte pergunta:

\footnotetext{
${ }^{2}$ Lembrar que este termo é uma tradução da palavra francesa naïve, que teóricos conjuntistas utilizam com a conotação de algo despreocupado ou pouco cauteloso em relação ao rigor lógico da Teoria Axiomática dos Conjuntos.
} 
Questão 3.1. É possível provar os resultados nos Teoremas 3.2.1 e 3.2.2 em ZF + UT?

Encerraremos este capítulo provando o próximo teorema, que é uma generalização do Teorema 3.2.2.

Teorema 3.2.3. Sejam I um conjunto não vazio, $\mathcal{U}$ um ultrafiltro sobre $I$ e $\left\{\left\langle X_{i}, \tau_{i}\right\rangle: i \in I\right\}$ uma família de espaços topológicos não vazios e de Tychonoff. Assumindo-se AC, tem-se que $\prod_{I, \mathcal{U}}\left\langle C_{b}\left(X_{i}, \tau_{i}\right),|\cdot|_{\infty, X_{i}}\right\rangle$ é isometricamente isomorfo a $\left\langle C\left(\sum_{I, \mathcal{U}} X_{i}, \bigoplus_{I, \mathcal{U}} \tau_{i}\right),|\cdot|_{\infty, \sum_{I, \mathcal{U}} X_{i}}\right\rangle$.

Prova. Assuma UT: em virtude do Comentário 3.1.9 (vi) e do Teorema 3.1.3 (ii), pode-se considerar, para cada $i \in I$, a função $\Psi_{i}$ que é definida como o único isomorfismo isométrico $\Psi: C_{b}\left(X_{i}, \tau_{i}\right) \rightarrow C\left(\beta X_{i}, \beta \tau_{i}\right)$ tal que, para toda função $\xi \in C_{b}\left(X_{i}, \tau_{i}\right)$,

$$
\Psi(\xi) \circ \beta=\xi \text {. }
$$

Considere então a família $\left\{\Psi_{i}: i \in I\right\} .{ }^{3}$ Em virtude do Teorema 2.2.30(ii)(2), fixe um isomorfismo isométrico

$$
\Phi: \prod_{I,|\cdot|_{\infty}, X_{i}, \mathcal{U}} C_{b}\left(X_{i}, \tau_{i}\right) \rightarrow \prod_{I,|\cdot|_{\infty, \beta X_{i}}, \mathcal{U}} C\left(\beta X_{i}, \beta \tau_{i}\right)
$$

segundo $\bigotimes_{I, \mathcal{U}}|\cdot|_{\infty, X_{i}}$ e $\bigotimes_{I, \mathcal{U}}|\cdot|_{\infty, \beta X_{i}}$.

Assuma AC: em virtude do Teorema 3.2.1, fixe um homeomorfismo

$$
\gamma: \sum_{I, \mathcal{U}} X_{i} \rightarrow \sum_{I, \mathcal{U}} \beta X_{i}
$$

com respeito a $\bigoplus_{I, \mathcal{U}} \tau_{i}$ e $\bigoplus_{I, \mathcal{U}} \beta \tau_{i}$. Em virtude dos itens $(v)(2)(2)$ e (ix) (2) do Comentário 1.2.37, fixe então um isomorfismo isométrico

$$
\Gamma: C_{b}\left(\sum_{I, \mathcal{U}} \beta X_{i}, \bigoplus_{I, \mathcal{U}} \beta \tau_{i}\right) \rightarrow C_{b}\left(\sum_{I, \mathcal{U}} X_{i}, \bigoplus_{I, \mathcal{U}} \tau_{i}\right)
$$

segundo $|\cdot|_{\infty, \sum_{I, \mathcal{U}} \beta X_{i}} \mathrm{e}|\cdot|_{\infty, \sum_{I, \mathcal{U}} X_{i}} \cdot$

É claro que, para todo $i \in I, \beta X_{i}$ é não vazio e $\beta\left\langle X_{i}, \tau_{i}\right\rangle$ é compacto. Em virtude dos itens $(v)(1)$ e (iv) (1) da Proposição 1.1 .16 e do Teorema 3.2.2, fixe um isomorfismo

${ }^{3}$ Esta parte da prova está, de fato, estabelecida em ZF + UT devido à unicidade de tais funções em relação às suas propriedades representadas no diagrama do Teorema 3.1.3 (ii). 
isométrico

$$
\Lambda: \prod_{I,\left.|\cdot|\right|_{\infty}, \beta X_{i}, \mathcal{U}} C\left(\beta X_{i}, \beta \tau_{i}\right) \rightarrow C\left(\sum_{I, \mathcal{U}} \beta X_{i}, \bigoplus_{I, \mathcal{U}} \beta \tau_{i}\right)
$$

segundo $\bigotimes_{I, \mathcal{U}}|\cdot|_{\infty, \beta X_{i}}$ e $|\cdot|_{\infty, \sum_{I, \mathcal{U}} \beta X_{i}}$.

Em virtude do Corolário 3.1.11 (ii) (2) e do Comentário 1.2.36 (iv) (2), considere então a função

$$
\Gamma \circ \Lambda \circ \Phi: \prod_{I,\left.|\cdot|\right|_{\infty}, X_{i}, \mathcal{U}} C_{b}\left(X_{i}, \tau_{i}\right) \rightarrow C\left(\sum_{I, \mathcal{U}} X_{i}, \bigoplus_{I, \mathcal{U}} \tau_{i}\right)
$$

É imediato concluir que $\Gamma \circ \Lambda \circ \Phi$ é um isomorfismo isométrico segundo $\bigotimes_{I, \mathcal{U}}|\cdot|_{\infty, X_{i}}$ e $|\cdot|_{\infty, \sum_{I, \mathcal{U}} X_{i}}$ Portanto, conclui-se que $\prod_{I, \mathcal{U}}\left\langle C_{b}\left(X_{i}, \tau_{i}\right),|\cdot|_{\infty}, X_{i}\right\rangle$ é isometricamente isomorfo a $\left\langle C\left(\sum_{I, \mathcal{U}} X_{i}, \bigoplus_{I, \mathcal{U}} \tau_{i}\right),|\cdot|_{\infty}, \sum_{I, \mathcal{U}} x_{i}\right\rangle$.

Note que, de fato, o Teorema 3.2.3 generaliza o Teorema 3.2.2, pois, em ZFC, prova-se a asserção

todo espaço topológico compacto e de Hausdorffé de Tychonoff com o uso da asserção

todo espaço topológico compacto e de Hausdorffé normal

(v. Teorema 17.10 de [Wil1970]) e do Lema de Urysohn (v. Corolário 15.7 de [Wil1970]), que podem ser demonstrados utilizando $\mathbf{A C}$. 


\section{Considerações Finais}

Com esta tese, estabelecemos

- generalizações de definições e resultados da teoria dos ultraprodutos de espaços métricos pontuados no contexto dos espaços pseudométricos.

- uma generalização da teoria dos ultraprodutos de espaços normados e de Banach reais ou complexos no contexto dos espaços pseudonormados sobre subcorpos quaisquer de $\mathbb{C}$.

Com essas generalizações, provamos,

- em ZF, ZF + AC e $_{\omega}$ ou ZFC, generalizações de resultados referentes a ultraprodutos de espaços métricos ou a ultraprodutos de espaços normados e de Banach.

A motivação inicial para o desenvolvimento deste trabalho foi a nossa intenção de obter, em ZF ou em uma extensão própria de $\mathbf{Z F}$, resultados de preservação ou exemplos que testemunhem a não preservação, sob ultraprodutos normados com respeito a um ultrafiltro livre, das propriedades ser isomorfo ao quadrado, ser indecomponível e ter poucos operadores no sentido de P. Koszmider (v. [Kos2004]).

Para a finalização desta tese,

- introduzimos detalhadamente, em ZF + UT, a teoria das ultrassomas de espaços topológicos de Tychonoff.

- provamos, em ZFC, uma generalização da Proposição 2 de [Gur1988] no contexto dos espaços topológicos de Tychonoff.

A definição de ultrassoma topológica foi introduzida neste trabalho devido ao nosso interesse em ter uma compreensão mais profunda das construções de ultracoprodutos 
topológicos apresentadas em [Ban1984, Ban1987], pois acreditamos que tais construções podem servir como ferramental para obter, em ZFC ou em uma extensão própria de ZFC, um exemplo de

um espaço topológico $\langle K, \tau\rangle$ compacto e de Hausdorff para o qual $K$ é infinito e o espaço de Banach $\left\langle C(K, \tau),|\cdot|_{\infty, K}\right\rangle$ é indecomponível e tem poucos operadores.

Exemplos de um tal espaço topológico foram apresentados por Koszmider em [Kos2004, Kos2013] e por R. A. S. Fajardo em [Faj2009] com a utilização da técnica de extensão por sequência disjunta de funções contínuas (v. [Kos2004]) ou do método de forcing (v. [Faj2009, Kos2013]).

Por fim, informamos que temos a intenção de estabelecer

- uma definição de ultraproduto métrico âncorado em conjuntos finitamente distais para famílias de espaços quase métricos quaisquer, tendo em vista a obtenção de resultados, no contexto desses espaços, sobre o completamento ou a completeza de ultraprodutos ou que generalizem a maioria daqueles apresentados na Seção 2.2 .

- uma versão do Teorema de Łoś para ultraprodutos de espaços quase métricos com respeito a um mesmo ultrafiltro e âncorado em um mesmo conjunto finitamente distal, tendo como inspiração o Teorema 5.4 de [B $\left.{ }^{+} 2008\right]$, que é a versão do Teorema de Łoś na lógica contínua de primeira ordem proposta por I. Ben Yaacov et al. 


\section{Referências Bibliográficas}

[Avi ${ }^{+}$2013] AVILÉS, A. et al. On ultrapowers of Banach spaces of type $\mathscr{L}_{\infty}$, Fundamenta Mathematicae, v. 222, n. 3, p. 195-212, 2013. 2, 117, 118

[Avi ${ }^{+}$2016] _. Separably injective Banach spaces. Cham: Springer, 2016. (Lecture Notes in Mathematics, 2132) 2

[Ban1984] BANKSTON, P. Expressive power in first order topology, The Journal of Symbolic Logic, v. 49, n. 2, p. 478-487, 1984. 2, 138, 142

[Ban1987] _. Reduced coproducts of compact Hausdorff spaces, The Journal of Symbolic Logic, v. 52, n. 2, p. 404-424, 1987. 2, 137, 142

[BeS1969] BELL, J. L.; SLOMSON, A. B. Models and ultraproducts: an introduction. Amsterdam: North-Holland, 1969. 1, 130

[B ${ }^{+}$2008] BEN YAACOV, I. et al. Model theory for metric structures. In: CHATZIDAKIS, Z. et al. (ed.). Model theory with applications to algebra and analysis: volume 2. Cambridge: Cambridge University Press, 2008. p. 315-427. 2, 101, 103, 142

[BuS1981] BURRIS, S.; SANKAPPANAVAR, H. P. A course in universal algebra. New York: Springer, 1981. (Graduate Texts in Mathematics, 78) 39

[CN1974] COMFORT, W. W.; NEGREPONTIS, S. The theory of ultrafilters. New York: Springer, 1974. (Grundlehren der mathematischen Wissenschaften: A Series of Comprehensive Studies in Mathematics, 211) 130

[Con1990] CONWAY, J. B. A course in functional analysis. 2nd. ed. New York: Springer, 1990. (Graduate Texts in Mathematics, 96) 5 
[DCK1972] DACUNHA-CASTELLE, D.; KRIVINE, J.-L. Applications des ultraproduits à l'étude des espaces et des algèbres de Banach, Studia Mathematica, v. 41, n. 3, p. $315-334,1972.1,117,118$

[DN2004] DOLECKI, S.; NOGURA, T. Convergence. In: HART, K. P.; NAGATA, J.; VAUGHAN, J. E. (ed.). Encyclopedia of general topology. Amsterdam: Elsevier Science, 2004. p. 18-21. 30

[End1977] ENDERTON, H. B. Elements of set theory. New York: Academic Press, 1977. 5

[Eng1989] ENGELKING, R. General topology. revised and completed. ed. Berlin: Heldermann, 1989. (Sigma Series in Pure Mathematics, 6) 5, 13, 22, 23, 135

[Faj2009] FAJARDO, R. A.S. An indecomposable Banach space of continuous functions which has small density, Fundamenta Mathematicae, v. 202, n. 1, p. 43-63, 2009. 142

[Gab2016] GABA, Y. O. K. U. Construction of quasi-metrics determined by orders. 2016. Tese (Doutorado em Matemática) - Faculty of Science, University of Cape Town, 2016. 25

[G1930] GÖDEL, K. Die Vollständigkeit der Axiome des logischen Funktionenkalküls. Monatshefte für Mathematik und Physik, v. 37, n. 1, p. 349-360, 1930. Translated in: VAN HEIJENOORT, J. From Frege to Gödel: a source book in mathematical logic, 1879-1931. Cambridge, MA: Harvard University Press, 1967. p. 582-591. 1

[Gur1988] GUREVIČ, R. On ultracoproducts of compact Hausdorff spaces, The Journal of Symbolic Logic, v. 53, n. 1, p. 294-300, 1988. 2, 3, 121, 137, 138, 141

[Gut2008] GUTIERRES, G. On countable choice and sequential spaces, Mathematical Logic Quarterly, v. 54, n. 2, p. 145-152, 2008. 45

[Hei1980] HEINRICH, S. Ultraproducts in Banach space theory, Journal für die reine und angewandte Mathematik, v. 1980, n. 313, p. 72-104, 1980. 2, 117, 118

[HI2002] HENSON, C. W.; IOVINO, J. Ultraproducts in analysis. In: FINET, C.; MICHAUX, C. (ed.). Analysis and logic. Cambridge: Cambridge University Press, 2002. p. 1-112. 1, 2 
[HK2015] HERRLICH, H.; KEREMEDIS, K. On the metric reflection of a pseudometric space in ZF, Commentationes Mathematicae Universitatis Carolinae, v. 56, n. 1, p. $77-88,2015.39,45$

[Hew1948] HEWITT, E. Rings of real-valued continuous functions. I, Transactions of the American Mathematical Society, v. 64, n. 1, p. 45-99, 1948. 1

[HR1998] HOWARD, P.; RUBIN, J. E. Consequences of the axiom of choice. Providence, RI: AMS, 1998. (Mathematical Surveys and Monographs, 59) 9

[Jec2003] JECH, T. J. Set theory: the third millennium edition, revised and expanded. Berlin: Springer, 2003. (Springer Monographs in Mathematics) 5

[JF2018] JESUS, J. P. C.; FAJARDO, R. A. S. Duas aplicações do teorema do ultrafiltro em análise funcional, Revista Matemática Universitária, n. 54, p. 33-45, 2018. Disponível em: <https://rmu.sbm.org.br/wp-content/uploads/sites/27/2018/ 08/Fajardo_e_Cirineu_final.pdf>. Acesso em: 15 jul. 2021. 9

[Kos2004] KOSZMIDER, P. Banach spaces of continuous functions with few operators, Mathematische Annalen, v. 330, n. 1, p. 151-183, 2004. 141, 142

[Kos2013] . On large indecomposable Banach spaces, Journal of Functional Analysis, v. 264, n. 8, p. 1779-1805, 2013. 142

[Kri1967] KRIVINE, J.-L. Sous-espaces et cones convexes dans les espaces $L^{p}$. 1967. Tese (Doutorado em Matemática) - Faculté des Sciences, Université de Paris, Paris, 1967. 1

[Kro1986] KROM, M. A linearly ordered topological space that is not normal, Notre Dame Journal of Formal Logic, v. 27, n. 1, p. 12-13, 1986. 17

[Los1955] ŁOŚ, J. Quelques remarques, théorèmes et problèmes sur les classes définissables d'algèbres. In: SKOLEM, T. et al. (ed.). Mathematical interpretation of formal systems. Amsterdam: North-Holland, 1955. p. 98-113. 1

[Lux1969] LUXEMBURG, W. A. J. A general theory of monads. In: (ed.). Applications of model theory to algebra, analysis, and probability. New York: Holt, Rinehart and Winston, 1969. p. 18-86. 1

[Meg1998] MEGGINSON, R. E. An introduction to Banach space theory. New York: Springer, 1998. (Graduate Texts in Mathematics, 183) 5, 54 
[NB2011] NARICI, L.; BECKENSTEIN, E. Topological vector spaces. 2nd. ed. Boca Raton, FL: CRC Press, 2011. (Pure and Applied Mathematics: A Series of Monographs and Textbooks)

[Rud1987] RUDIN, W. Real and complex analysis, 3rd. ed. New York: McGraw-Hill, 1987. (McGraw-Hill Series in Higher Mathematics) 54

[Sch1997] SCHECHTER, E. Handbook of analysis and its foundations. San Diego, CA: Academic Press, 1997. 9, 135

[She2009] SHERMAN, D. Notes on automorphisms of ultrapowers of $\mathrm{II}_{1}$ factors, Studia Mathematica, v. 195, n. 3, p. 201-217, 2009. 1

[Sko1934] SKOLEM, T. Über die Nicht-charakterisierbarkeit der Zahlenreihe mittels endlich oder abzählbar unendlich vieler Aussagen mit ausschliesslich Zahlenvariablen, Fundamenta Mathematicae, v. 23, n. 1, p. 150-161, 1934. 1

[Ste1970] STEEN, L. A. A direct proof that a linearly ordered space is hereditarily collectionwise normal, Proceedings of the American Mathematical Society, v. 24, n. 4 , p. $727-728,1970.17$

[SS1978] ; SEEBACH JR., J. A. Counterexamples in topology, 2nd. ed. New York: Springer, 1978. 17

[S1978] STERN, J. Ultrapowers and local properties of Banach spaces, Transactions of the American Mathematical Society, v. 240, p. 231-252, 1978. 2, 51, 117

[Tar1930] TARSKI, A. Une contribution à la théorie de la mesure, Fundamenta Mathematicae, v. 15, n. 1, p. 42-50, 1930. 9

[VD1985] VAN DOUWEN, E. K. Horrors of topology without AC: a nonnormal orderable space, Proceedings of the American Mathematical Society, v. 95, n. 1, p. 101-105, 1985. 17

[V1995] VINOGRADOVA, I. A. Borel-Lebesgue covering theorem. In: HAZEWINKEL, M. (ed.). Encyclopaedia of Mathematics: volume 1. Dordrecht: Kluwer Academic, 1995. p. 437.41

[Wil1970] WILLARD, S. General topology. Reading, MA: Addison-Wesley, 1970. 5, 23, 140 
[WJ1987] WILLIAMSON, R.; JANOS, L. Constructing metrics with the Heine-Borel property, Proceedings of the American Mathematical Society, v. 100, n. 3, p. 567$573,1987.43$

[W1954] WRIGHT, F. B. A reduction for algebras of finite type, Annals of Mathematics, sec. ser., v. 60, n. 3, p. 560-570, 1954. 1 



\section{Índice Remissivo}

\section{B}

base

de filtro 6-8

local 12, 13, 31, 68

C

classe

de equivalência $11,84,87$

compactificação

de Stone-Čech 136, 137

maximal 122-124, 127, 133

usual 133,137

compactificado 122, 136

de Stone-Čech 136

maximal 122, 133, 136

conjunto

denso (com respeito a uma

topologia) 14, 17, 18, 22, 31, 49,

$121,124,125,129,132$

denso (segundo uma pré-ordem)

$11,17,79$

finitamente distal $81,82,84,85,87$, $89,90,92,93,102,104-108$,

$110-112,114$

limitado 39, 40 que contém qualquer constante 81 , 106, 107

quociente 11,50

sequencialmente fechado $44,52,56$

sup-finitamente distal 92-94, 96, 114-116

coproduto

reduzido

topológico 137

corpo

topológico $16,18,53,54,76$

D

diâmetro 40

dimétrica 25, 27, 28, 34-36, 38, 85

dimensão

de Menger-Urysohn 13

direção 10

E

espaço

de Banach 52, 56

de Stone 131

métrico

discreto 29 


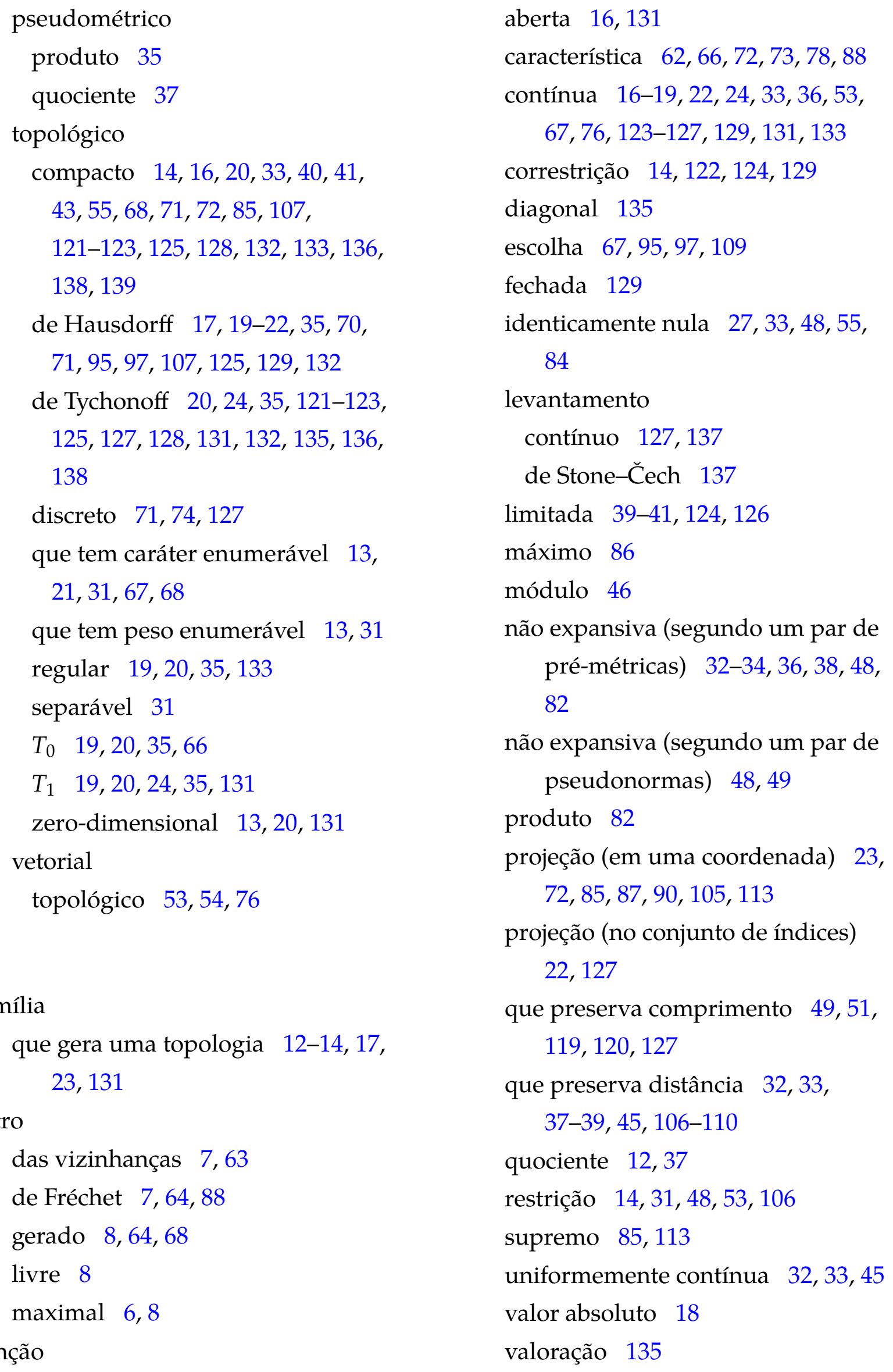


$\mathbf{H}$

hemimétrica 25-33, 35-38, 40-44, 57,

$$
64,83,85
$$

completa 44,45

homeomorfismo $16,24,57,58,123$, $124,128,129,136,139$

\section{I}

imersão

isométrica $32,39,57$

topológica 16, 121, 122, 131, 136

isometria $33,37,57,108$

isomorfismo

isométrico $49,58,119,124,127$, $135,139,140$

L

Lema

de Urysohn 140

M

métrica

completa 45

de Heine-Borel 43

limitada 89,90

própria 43

quociente 39

usual 29

zero-um 28

múltiplo

reduzido

topológico 137

medida

de contagem 54

não negativa 54

$\sigma$-aditiva 54

monomorfismo isométrico $49,51,58$

$\mathbf{N}$

network 12, 30, 31

local $12,13,31$

norma

euclidiana 51

quociente 51

O

ordem

parcial $10,12,78,79$

total $10,17,18,29,79$

$\mathbf{P}$

potência

cartesiana $51,55,78,83$

topológica $74,135,136$

pré-

métrica $25,26,28,29,31,32,34,35$, $37,38,64,81-83,85$ completa $43,44,95,96,99,101$ de Heine-Borel 43 induzida 31,85 invariante por sucessão 37 limitada 39 produto 35 quociente 37 ordem $10-12,38,78,83$ total $10,12,79$

primeiro modelo de Cohen 9 de Pincus-Solovay 9,66

Primeiro Teorema do Isomorfismo 39, 51

produto cartesiano $23,51,72,74,81,85,87$, 
89, 92-94, 97, 101, 104, 105, 107,

111

topológico 14,23

propriedade

da interseção

finita $5-9,14,64,66,69,70,130$, 134

pseudo-

métrica 26-28, 30, 31, 33-36, 38-41, $44,45,47,48,57,83-87$

completa $45,52,94,97$

induzida 48

invariante por sucessão 39,50

nula 27,30

norma $46-48,50,55,58,111,113$

completa 52, 55, 116

da distância 50

induzida 48,113

nula 48

Q

quase

compactificação 122, 127

compactificado 122, 136

métrica 25-28, 36, 83, 86

pseudométrica 25

\section{$\mathbf{R}$}

rede

eventualmente constante 65

redução

reflexiva 11,17

reflexão

métrica 39

relação

estar finitamente distante 81,82, 84, $96,100,102,106,107,110,111$ de congruência 50, 111

de dominação 78

de equivalência $10-12,37,38,83$,

111

induzida (por uma pré-métrica)

38,87

induzida (por uma relação binária)

12,91

\section{S}

sequência

de Cauchy 41-43, 45, 95, 96, 99

eventualmente constante $20,73,88$

limitada 42

nula $77,80,83$

$\sigma$-álgebra 54

de Borel 54

soma

conjuntista 22

reduzida

topológica 137

topológica 22

$\mathbf{T}$

$T_{0}$-quase métrica 25

Teorema

da Compacidade 9

da Compactificação de Stone-Čech

9

da Representação de Gelfand 2

da Representação de Stone 9

de Banach-Alaoglu 9

de Heine-Borel 41

do Produto de Tychonoff 9

do Ultrafiltro 9

topologia

antidiscreta 29 
da ordem 17, 29

de Sierpiński 30

de Stone 131

de subespaço 15

discreta 17, 20, 29

gerada $12,14,17,23,131$

induzida 29,48

produto 14,23

soma 22

usual 19

U

ultra-

coproduto

topológico 137

filtro $6-9,60-62,66,68,69,71,79$, $85,87,89,91-93,97,98,101$, 104-108, 110, 112, 114-119, 130, $134,137-139$

livre $9,66,72,86-88$

principal $6,8,60,63,66,71,78$,
$87,105,130,131$

hemimétrica 25-28, 32, 34, 36-38, $77,83,86$

métrica 26-28, 36, 86

múltiplo

topológico 137

norma 46,47

potência

métrica 104

normada 117,118

produto

métrico 104

normado 117

pseudométrica $26-28,47,77,86$

pseudonorma $46-48,51,113,114$

soma

topológica 137

V

vizinhança

canônica $14,23,74,75$ 